\title{
Kinetische und thermodynamische Untersuchungen der Hochdruck-Copolymerisation von Ethen mit (Meth)Acrylsäureestern
}

\author{
Dissertation zur Erlangung des Doktorgrades \\ der Mathematisch-Naturwissenschaftlichen Fakultäten \\ der Georg-August-Universität zu Göttingen
}

vorgelegt von

\section{Henning Latz}

aus

Düren

Göttingen 2004 
D 7

Referent:

Prof. Dr. M. Buback

Korreferent:

Prof. Dr. K. Hoyermann

Tag der mündlichen Prüfung:

29.04.2004 


\section{Inhaltsverzeichnis}

1. Zusammenfassung 1

2. Einleitung 4

3. Materialien und Methoden 9

3.1 Studium von Copolymerisationsreaktionen 9

3.1.1 Verwendete Chemikalien 9

3.1.2 Experimentelle Anordnung zur kontinuierlichen Hochdruck-Copolymerisation 10

3.1.3 Auswahl der Messbedingungen 16

3.1.4 Durchführung von Copolymerisationsexperimenten 18

3.1.5 Aufarbeitung der Copolymerproben 19

$\begin{array}{lll}3.2 & \text { Copolymeranalyse } & 21\end{array}$

3.2.1 Bestimmung der Copolymerzusammensetzung mittels ${ }^{1} \mathrm{H}-\mathrm{NMR}$ Spektroskopie

3.2.2 Bestimmung der Copolymerzusammensetzung mittels Elementaranalyse

3.2.3 Bestimmung der Zusammensetzung von Ethen-Methacrylsäure$\begin{array}{ll}\text { Butylmethacrylat-Terpolymeren } & 27\end{array}$

3.2.4 FT-IR-spektroskopische Untersuchungen 31

3.2.4.1 Univariate NIR/IR-NMR-Korrelation 33

3.2.4.2 Multivariate NIR/IR-NMR-Korrelation 42

3.2.5 Bestimmung der Molekulargewichtsverteilungen 48

3.3 Trübungsdruckmessungen an Ethen / Polymer-Systemen 50

3.3.1 Verwendete Substanzen $\quad 50$

3.3.2 Experimentelle Anordnung für diskontinuierliche Messungen 51

3.3.3 Durchführung von Trübungsdruckmessungen mit der Satzzelle 55

3.3.4 Reinigung 56

3.3.5 Experimentelle Anordnung für kontinuierliche Messungen 56

3.3.6 Auswahl der Messbedingungen 58

$\begin{array}{llr}3.4 & \text { Fehlerbetrachtung } & 59\end{array}$

3.4.1 Unsicherheit der Messgeräte $\quad 59$

$\begin{array}{lll}3.4 .2 & \text { Weitere Fehlerquellen } & 60\end{array}$

$\begin{array}{lll}3.5 & \text { Literatur Kapitel } 3 & 62\end{array}$ 
4. Ethen-(Meth)Acrylsäure-Copolymerisationsparameter 64

4.1 Grundlagen der Copolymerisationskinetik 64

4.2 Definition von Copolymerisationsparametern 66

4.3 Bestimmumg von Ethen-(Meth)Acrylat-Copolymerisationsparametern 75

4.3.1 Bestimmung der Copolymerzusammensetzung 75

4.3.2 Bestimmung der Zusammensetzung der Comonomermischung 75

4.4 Gewichtung der Daten - Berechnung der Fehlerstruktur 77

4.5 Bestimmung von Copolymerisationsparametern - Ergebnisse 83

4.5.1 Ethen-Acrylat-Copolymersysteme 83

$\begin{array}{lll}\text { 4.5.2 Ethen-Methacrylat-Copolymersysteme } & 88\end{array}$

4.6 Ethen-(Meth)Acrylat-Copolymerisationsparameter -
Vergleich mit Literaturdaten

$\begin{array}{lll}4.7 & \text { Literatur Kapitel } 4 & 102\end{array}$

5. Untersuchungen zum Entmischungsverhalten 104

5.1 Entmischungsverhalten von

Polymer-Solvens-Systemen - Phänomenologie 104

5.1.1 Phasendiagramme binärer Mischungen 104

5.1.2 Phasenverhalten von Mischungen aus Polymer und Solvens 106

$\begin{array}{lll}5.2 & \text { Bestimmung von Trübungsdrücken } & 109\end{array}$

5.3 Validierung der experimentellen Methode zur

Messung von Trübungsdrücken

5.3.1 Untersuchungen zur Stabilität von Mischungen aus fluidem Ethen und Polymeren

5.3.2 Einfluss durch Verwendung eines Inhibitors 116

$\begin{array}{ll}\text { 5.3.3 Einfluss der Polymerkonzentration } & 117\end{array}$

5.3.4 Reproduzierbarkeit der Trübungsdruckmessungen mit der kontinuierlichen Apparatur

5.4 Ergebnisse der Trübungsdruckmessungen an binären Mischungen aus Ethen und Ethen-(Meth)Acrylat-Copolymeren

5.4.1 System Ethen / Ethen-Ethylacrylat-Copolymer 119

5.4.2 System Ethen / Ethen-Propylacrylat-Copolymer 121

5.4.3 System Ethen / Ethen-Methylmethacrylat-Copolymer 123

5.4.4 System Ethen / Ethen-Butylmethacrylat-Copolymer 125

5.4.5 System Ethen / Ethen-Benzylmethacrylat-Copolymer 126 
5.5 Kontinuierliche Trübungsdruckmessungen mit der, ,inline“-Methode

5.5.1 System

Ethen / Ethylmethacrylat / Ethen-Ethylmethacrylat-Copolymer

5.5.2 System

Ethen / Benzylmethacrylat / Ethen-Benzylmethacrylat-Copolymer 130

5.6 Ergebnisse der Trübungsdruckmessungen an Mischungen aus Ethen und Ethen-Methacrylsäure-Butylmethacrylat-Terpolymeren

$5.7 \quad$ Literatur Kapitel 5

6. Modellierungen mit der PC-SAFT-Zustandsgleichung 143

$\begin{array}{lll}\text { 6.1 Einleitung } & 143\end{array}$

6.2 Ergebnisse 146

6.2.1 System Ethen / Ethen-Methylacrylat-Copolymer. 146

6.2.2 System Ethen / Ethen-Ethylacrylat-Copolymer 150

6.2.3 System Ethen / Ethen-Propylacrylat-Copolymer 151

6.2.4 System Ethen / Ethen-Butylacrylat-Copolymer 153

6.2.5 System Ethen / Ethen-Methylmethacrylat-Copolymer 156

6.2.6 System Ethen / Ethen-Butylmethacrylat-Copolymer 157

6.2.7 Abhängigkeit des Trübungsdrucks von der Molmasse 163

$\begin{array}{lll}\text { 6.3 } & \text { Literatur Kapitel 6 } & 166\end{array}$

$\begin{array}{ll}\text { 7. Ausblick } & 167\end{array}$

Anhang

A Verzeichnis der Abkürzungen

B Aufstellung der untersuchten Polymere

C Aufstellung der experimentellen Ergebnisse zum Entmischungsverhalten

D Parameter für die Berechnung von Entmischungsdrücken mit PC-SAFT 


\section{Zusammenfassung}

Es wurden kinetische und thermodynamische Aspekte der radikalischen HochdruckCopolymerisation von Ethen mit (Meth)Acrylsäureestern ((Meth)Acrylaten) in einem weiten Zustandsbereich untersucht.

Unter kontinuierlicher Reaktionsführung wurden in einem nahezu ideal durchmischten Rührkessel bei einem Druck von 2000 bar und Temperaturen zwischen 170 und $240{ }^{\circ} \mathrm{C}$ in homogener Phase Co- und Terpolymerisationen von Ethen mit (Meth)Acrylsäure(estern) ausgeführt. Hierbei wurden für die weiteren Untersuchungen Polymerproben aus Copolymerisationen von Ethen mit Ethylacrylat (E-EA), mit Propylacrylat (E-PA), mit Methylmethacrylat (E-MMA), mit Ethylmethacrylat (E-EMA), mit Butylmethacrylat (E-BMA), mit Benzylmethacrylat (E-BzMA) und mit Methacrylsäure und Butylmethacrylat (E-MAS-BMA) aus einem weiten Zusammensetzungsbereich erhalten.

Der (Meth)Acrylatgehalt der Copolymere wurde ${ }^{1} \mathrm{H}-\mathrm{NMR}$-spektroskopisch und zur Verifizierung durch Elementaranalysen bestimmt. Es wurden NMR-IR-Korrelationen aufgestellt und an diesen gezeigt, dass sich die Genauigkeit der IR-spektroskopischen Zusammensetzungsanalyse mit einer multivariaten anstelle einer univariaten Methode erheblich steigern lässt. Die Molekulargewichtsverteilung der Copolymere wurde mittels Gelpermeationschromatografie ermittelt.

Die kinetische Analyse der Verknüpfung von Monomer- und Copolymerzusammensetzung gibt keinen Hinweis auf Penultimate-Effekte. Das Copolymerisationsverhalten der verschiedenen Ethen-Acrylat-Systeme ist nicht unterscheidbar. Auch für die Ethen-MethacrylatSysteme wird keine Abhängigkeit der Zusammensetzungskinetik von der Art des Alkylesterrests der Comonomere beobachtet. Im Vergleich der Ethen-Methacrylat- mit den EthenAcrylat-Systemen zeigt sich, dass Methacrylate stärker ins Polymer eingebaut werden. Der Unterschied nimmt mit abnehmender Temperatur und zunehmenden Anteil (Meth)Acrylat in der Comonomermischung zu.

Auf Basis des Terminal- / Impliziten-Penultimate-Modells wurden Copolymerisationsparameter bestimmt. Die für die Ethen-Acrylat- und Ethen-Methacrylat-Systeme erhaltenen $r_{\mathrm{E}^{-}}$ Werte und ihre Temperaturabhängigkeit sind nicht unterscheidbar und stimmen gut mit den Literaturdaten für Ethen-Methylacrylat- (E-MA), Ethen-Butylacrylat- (E-BA), E-MMA- und E-BMA-Copolmyere überein. Die Aktivierungsenergie der $r_{\mathrm{E}}$-Werte lässt sich aus Literaturdaten zur Aktivierungsenergie der Additionsreaktion des Methylradikals an Ethen 
und an die Monomere Methylacrylat (MA) und Methylmethacrylat (MMA) erstaunlich gut vorhersagen.

Die $r_{\mathrm{A}}$-Werte der untersuchten Ethen-Methacrylat-Systeme sind größer als die der EthenAcrylat-Systeme. Die erhaltenen $r_{\mathrm{A}}$-Werte stimmen innerhalb der Genauigkeit der Bestimmungsmethode mit den Literaturdaten anderer Ethen-Methacrylat- bzw. Ethen-AcrylatSysteme überein. Die aus den Additionsreaktionen von MMA- und MA-ähnlichen Modellradikalen (2-tert-butoxycarbonyl-2-propyl- bzw. tert-butoxycarbonylmethyl-Radikal) an die Monomere MMA bzw. MA und Ethen abgeschätzten Aktivierungsenergien der untersuchten Ethen-Methacrylat-Systeme stimmen ebenfalls überein.

In weiten Bereichen von Druck, Temperatur und der Copolymerzusammensetzung wurden unter Anwendung einer mit einem Saphirfenster ausgestatteten Hochdruckzelle mit variablem Volumen Trübungsdruckdaten für Mischungen aus Ethen und verschiedenen Copolymeren (E-EA, E-PA, E-MMA, E-BMA, E-BzMA und E-MAS-BMA) bestimmt. Die Mischungen wurden aufgrund der starken Polymerisationsneigung unter Messbedingungen mit einem Inhibitor stabilisiert. Eine Verfälschung der Ergebnisse durch intermediäre chemische Reaktionen konnte durch IR- und ${ }^{1} \mathrm{H}-\mathrm{NMR}$-spektroskopische Analyse sowie durch Bestimmung der Molekulargewichtsverteilung der Copolymere jeweils vor und nach den Experimenten ausgeschlossen werden.

Die Trübungsdrücke von E-EA und E-PA, E-MMA und E-BMA werden ausgehend von reinem Polyethylen mit zunehmendem (Meth)Acrylatanteil bis zu einem Gehalt von etwa 25 mol-\% abgesenkt. $\mathrm{Zu}$ höheren Gehalten findet man für E-MMA und E-BMA einen starken, für E-EA einen schwächer ausgeprägten und für E-PA keinen Anstieg der Trübungsdrücke. Die Trübungsdrücke der Systeme E/E-EA, E/E-PA und E / E-BA sind bei Copolymer-Acrylatgehalten unter 20 mol-\% im Rahmen der Messgenauigkeit nicht unterscheidbar. Zu höheren Acrylatgehalten hin liegt der Trübungsdruck umso niedriger je größer der Alkyl-Esterrest ist.

Im Vergleich der Ergebnisse für Ethen-Acrylat- und Ethen-Methacrylat-Copolymere zeigt sich bis zu einer Copolymerzusammensetzung von ca. 40 mol-\% ein löslichkeitsverbessernder Effekt durch das Vorhandensein der $\alpha$-Methylgruppe. Die für E-MMA bestimmten Trübungsdrücke sind etwa 100 bar niedriger als für entsprechende E-MA-Copolymere gleichen Gehalts. Auch für die E-B(M)A-Copolymer-Systeme werden bis zu einer Copolymerzusammensetzung von ca. 25 mol-\% für E-BMA-Copolymere im Vergleich zu E- 
BA-Copolymeren geringere Trübungsdrücke beobachtet. $\mathrm{Zu}$ höheren Comonomergehalten zeigt sich hier jedoch eine löslichkeitsverringernde Wirkung der $\alpha$-Methylgruppe.

Für E-BzMA-Copolymere wurden mit zunehmendem Comonomergehalt stark ansteigende Trübungsdrücke gefunden. Die im Vergleich sehr hohen Trübungsdrücke resultieren aus besonders starken $p \pi-p \pi($ Segment-Segment)-Wechselwirkungen (sog. „Stacking“) der Benzylgruppen. Diese Wechselwirkung konnte ${ }^{1} \mathrm{H}$-NMR-spektroskopisch nachgewiesen werden. Für die ternären Systeme Ethen / Ethylmethacrylat / Ethen-Ethylmethacrylat-Copolymer und Ethen / Benzylmethacrylat / Ethen-Benzylmethacrylat-Copolymer wurden unter praxisnahen Bedingungen mit einer kontinuierlichen Methode in direktem Anschluss an die Synthese Trübungsdrücke aufgenommen. Hierbei konnte die Abhängigkeit des Trübungsdrucks vom Methacrylatanteil im Copolymer als auch vom Anteil in der Comonomermischung bestimmt werden.

In Kooperation mit Frau Prof. G. Sadowski und Frau Dipl. Ing. F. Tumakaka (Universität Dortmund) wurde eine auf Copolymer-Solvens-Systeme erweiterte Zustandsgleichung (Perturbed Chain-SAFT-Zustandsgleichung) zur modellierenden Beschreibung der experimentellen Ergebnisse genutzt. Ausgehend von unabhängig bestimmten Reinstoffparametern und den durch Anpassung an die jeweiligen Randsysteme (Ethen/Polyethylen und Ethen / Poly(Meth)Acrylat) erhaltenen Parametern ermöglichte das Modell in weiten Grenzen von Druck, Temperatur und Copolymerzusammensetzung bei Verwendung nur eines (angepassten) binären Wechselwirkungsparameters eine gute Beschreibung der experimentellen Daten. Für diesen Parameter wurde für die Systeme E / E-EA, E / E-PA ein jeweils konstanter und für die Systeme E / E-MMA, E / E-BMA und E / E-MA ein linear von der Copolymerzusammensetzung abhängiger Wert bestimmt. Für das System E/E-BA gelingt die Modellierung mit einem zu Null gesetzten Wechselwirkungsparameter.

Die PC-SAFT-Gleichung erwies sich zudem als geeignet, die Abhängigkeit der Trübungsdrücke von der Molmasse in guter Übereinstimmung mit den experimentellen Ergebnissen wiederzugeben. 


\section{Einleitung}

Die Ära der Kunststoffe begann mit der Entdeckung des ersten gebrauchsfähigen vollsynthetischen Polymers durch Baekeland [1] im Jahre 1907.

Zunächst galten die Produkte der Polymerchemie noch als einfache Ersatzmaterialien für Naturprodukte wie Naturkautschuk, Seide oder Elfenbein. Doch schon bald nach der Entschlüsselung des Bauprinzips durch Staudinger in den 20er Jahren hat sich die Polymerforschung derart rasant fortentwickelt, dass es heute moderne Polymere sind, welche als Schrittmacher bei der Entwicklung von Schlüsseltechnologien fungieren. Die Anwendung von Kunststoffen hat einen ungeheuren Aufschwung erlebt. Allein in den letzten 30 Jahren hat sich die weltweite Kunststoffproduktion etwa verzehnfacht und erreicht heute die 200Millionen-Jahrestonnen-Marke [2].

Der wohl wichtigste Grund für den großen Erfolg synthetischer Polymere liegt, neben der vergleichsweise kostengünstigen Produktion, in der außergewöhnlichen Vielseitigkeit ihrer Produkteigenschaften, Anwendungen und Wiederverwertungsmöglichkeiten.

Je nach Beschaffenheit der molekularen und supermolekularen Struktur können Kunststoffe stahlartig fest, weich oder gummielastisch, elektrisch isolierend oder hochleitend, transparent oder lichtundurchlässig, langzeitstabil oder bioabbaubar sein, wobei auch ungewöhnliche Kombinationen von Eigenschaften verschiedener klassischer Werkstoffe möglich sind; als Beispiel lassen sich flüssigkristalline Elastomere anführen, welche sowohl die optischen Eigenschaften von Quarzeinkristallen als auch die Elastizität von Gummimaterialien aufweisen [3].

Die Vielseitigkeit ergibt sich aus der großen Anzahl an Freiheitsgraden bei der Ausgestaltung der Polymerstruktur. Auf der untersten Ebene steht die Wahl der chemischen Konstitution des kleinsten Polymerbausteins, des sogenannten Monomeren. Weitere Wahlmöglichkeiten betreffen die stereochemische Struktur (Taktizität) und den topologischen Aufbau, d.h. die Art der Verknüpfung der Monomere, zum Beispiel zu linearen oder verzweigten, kammförmigen, sternförmigen, dendritischen oder quervernetzten Strukturen. Hinzu kommt, dass die Materialeigenschaften häufig auch von der Art der Verteilung einzelner Strukturmerkmale beeinflusst werden können.

Die Vielfalt wird nochmals potenziert durch weitere Möglichkeiten der Modifikation der Eigenschaften, z.B. durch physikalisches Mischen (Blending) verschiedener Polymere und durch spezielle Verarbeitungstechniken wie die Erzeugung von Materialien mit Faser- 
verstärkung, von Verbundwerkstoffen, anorganischen-organischen Hybridmaterialien und Überstrukturen. Entsprechend kann heute für nahezu jede denkbare Anwendung ein maßgeschneiderter Polymerwerkstoff entwickelt werden.

Eine der einfachsten und gleichzeitig wirksamsten Möglichkeiten, die Eigenschaften eines Polymers zielgerichtet abzustimmen, ist die Copolymerisation. Das Prinzip dabei ist, Polymermoleküle aus zwei oder mehr verschiedenen Monomerbausteinen zusammenzusetzen.

Beispiele für im Großmaßstab produzierte Copolymere sind die nach dem PolyethylenHochdruckverfahren [6] hergestellten Copolymere von Ethen mit (Meth)Acrylsäure(ester). Diese Materialklasse besitzt aufgrund ihres mit steigendem Comonomergehalt abnehmenden Schmelzpunktes besonders gute Tieftemperaturstabilität, Spannungsrissbeständigkeit und hat aufgrund ihrer intensiven Haftung auf Metallen, Papier, Glas und Kunststoffen hervorragende Versiegelungseigenschaften. Die Anwendungsmöglichkeiten dieser Produkte reichen von (rußgefülltem) Material für Schläuche zum Schutz vor elektrostatischer Aufladung bis zu Zwischenfolien in Sicherheitsscheiben $[4,5]$.

Beim Verfahren der radikalischen Hochdruck-(Co)Polymerisation arbeitet man bei Temperaturen von 150 bis $300^{\circ} \mathrm{C}$ und Drücken von 1500 bis 3500 bar [6]. Für eine effiziente und sichere Produktion von ethenbasierten Copolymeren nach dem Hochdruckverfahren sind zwei Faktoren von zentraler Bedeutung: Zum einen sind Informationen zur Abhängigkeit der Copolymer- von der Comonomerzusammensetzung für das betrachtete Copolymersystem wichtig. Zum anderen ist es unerlässlich das Phasenverhalten der Reaktionsmischung bestehend aus Monomer und Polymer genau zu kennen. Dies hat folgende Gründe:

Im Reaktor sollte in der Regel eine Phasentrennung vermieden werden, da dies zum einen die Produktqualität beeinträchtigt und zum anderen die Reaktionsführung erschwert. Die ausfallende, polymerreiche Phase kann Wandbeläge bilden („Reaktorfouling“). Diese behindern die Wärmeabfuhr der stark exothermen Reaktion und lassen die Gefahr einer Wärmeexplosion entstehen. Die Abtrennung der Produkte erfordert wiederum eine gezielte Phasenseparation während mehrstufiger Entspannung der Reaktionsmischung in speziellen Abscheidern [7]. Zudem ist für einige Copolymersysteme bekannt, dass die Produkteigenschaften auch bei einer Synthese innerhalb des homogenen Zustandsgebiet von der Entfernung der Synthesebedingungen zur Entmischungsgrenze abhängen [8].

Das Phasenverhalten von Mischungen aus Polymeren und überkritischen Fluiden ist außerdem für verschiedene weitere Anwendungen interessant. An dieser Stelle sei 
exemplarisch die Möglichkeit der Fraktionierung [9], Aufreinigung und Trennung von Polymeren [10, 11], die Depolymerisation zur Rückgewinnung von Monomeren [12] und die Herstellung besonders kleiner Polymerpartikel erwähnt [13].

Ergebnisse aus der Literatur zur Zusammensetzungskinetik von Ethen(Meth)Acrylsäure(ester)-Copolymeren haben deutlich gemacht, dass sich Ethen-Acrylat-Systeme von den entsprechenden Ethen-Methacrylat-Systemen unterscheiden. $\mathrm{Zu}$ möglichen Unterschieden zwischen den einzigen bisher aus der Gruppe der Ethen-Acrylat-Copolymere untersuchten Systemen Ethen-Methylacrylat, Ethen-Butylacrylat- und Ethen-2-Ethylhexylacrylat werden widersprüchliche Angaben gemacht $[14,15]$.

In vorangegangenen Untersuchungen $\mathrm{zu}$ Copolymerisationen von Ethen mit (Meth)Acrylsäure(estern) $[16,17,18]$ haben sich bezüglich des Phasenverhaltens auffällige Unterschiede zwischen den Copolymeren mit (Meth)Acrylsäure, Methylacrylat und Butylacrylat gezeigt. Außerdem wurde für die säurehaltigen Copolymere festgestellt, dass auch die Änderung in der Polymerstruktur durch den Einbau von $\alpha$-Methylgruppen (bei den EthenMethacrylsäure-Copolymeren) das Entmischungsverhalten beeinflusst [18].

Für ein tieferes Verständnis des Zusammenhangs zwischen Comonomerstruktur und Trübungsdruck erschien es daher wichtig, in systematischen Untersuchungen des Entmischungsverhaltens weiterer Ethen-Copolymer-Systeme den Einfluss der Kettenlänge des Esters sowie den Einfluss der $\alpha$-Methylgruppe (bei den Methacrylsäureester) zu bestimmen. Darüber hinaus sollte es sich lohnen, die Auswirkungen von Funktionalitäten am Esterrest wie z.B. eine Benzyl-Gruppe auf das Phasenverhalten zu untersuchen.

Hierbei ist eine weitere interessante Fragestellung, ob die außerordentlich hohen Trübungsdrücke der Ethen-(Meth)Acrylsäure-Copolymere durch den zusätzlichen Einbau von (Meth)Acrylatbausteinen verringert werden können. Dazu sollte ein (Meth)Acrylat-Comonomer verwendet werden, welches beim Einbau in Ethen-Copolymere besonders niedrige Trübungsdrücke bewirkt.

Für die experimentellen Untersuchungen soll eine optische Hochdruckzelle mit variablem Innenvolumen verwendet werden. Diese gestattet es, das Entmischungsverhalten durch ein Saphirfenster visuell zu verfolgen und dabei Trübungspunkte bis zu Drücken von 3000 bar und Temperaturen von $260^{\circ} \mathrm{C}$ zu bestimmen.

Zum Erhalt möglichst exakter Daten für die Validierung bestehender theoretischer Modelle zur Beschreibung des Entmischungsverhaltens (s.u.) sollen für die experimentellen 
Messungen Copolymerproben mit einer schmalen Molekulargewichtsverteilung und möglichst einheitlicher Verteilung der Comonomereinheiten im Polymer eingesetzt werden. Diese können aus einer Synthese in einem kontinuierlich betriebenen Rührkessel einer Mini-Technikumsanlage unter definierten Bedingungen erhalten werden.

Experimentelle Messungen zu Hochdruckphasengleichgewichten sind mit einem hohen Aufwand verbunden. Da der Trübungsdruck zudem von einer Vielzahl von Variablen wie der Temperatur, der Molmasse, der Konzentration und Zusammensetzung der polymeren Mischungskomponente und von der Art und Konzentration an Cosolvens bestimmt ist, erscheint es sinnvoll ein universales theoretisches Modell aufzufinden, mit welchem die experimentellen Beobachtungen ausreichend präzise beschrieben und möglichst auch Vorhersagen getroffen werden können. Auf dem Gebiet der Mischungsthermodynamik mit polymeren Komponenten haben sich Zustandsgleichungen der SAFT-Familie (StatisticalAssociating-Fluid-Theory) besonders bewährt. Eine neuere Variante ist die Perturbed-ChainSAFT-Zustandsgleichung (PC-SAFT), welche speziell die Behandlung von Copolymeren berücksichtigt und bereits gute Resultate für Copolymere aus unpolaren Comonomeren ergeben hat [19]. In der vorliegenden Arbeit soll am Beispiel der Ethen-(Meth)Acrylat-Copolymere untersucht werden, ob das PC-SAFT-Modell auch zur Beschreibung von Copolymeren mit polaren Comonomeren geeignet ist. Darüber hinaus soll die Qualität von mit dem Modell getroffenen Vorhersagen geprüft werden. 


\section{Literatur zur Einleitung}

[1] L. H. Baekeland, US Patent 942699 (1907)

[2] Verband Kunststofferzeugende Industrie, Mitteilung zum Fachpressetag (03/2003)

[3] R. Mühlhaupt, Degussa Science Newsletter, 05 (2003)

[4] ExxonMobil (2000), http://www.exxon.mobil.com/chemical/cus...es/ldpe_copolymers/hperf_amer_grade.html

[5] M. Knights, Plastics Technology online article (2001) http://www.plastictechnology.com/articles/200111cu2.html

[6] "Ullmann`s encyclopedia of industrial chemistry", 5. ed. Vol. A 21 Weinheim (1992)

[7] B. Folie, M. Radosz, Ind. Eng. Chen. Res. 34 (1995) 1501

[8] O. K. McKinney, D. Eversdyk, D. Flores, Interpolymers of ethylene and unsaturated carboxylic acids, International Patent WO84/ 04926

[9] J. A. Pratt, M. A. McHugh, J. Supercritical Fluids 9 (1996) 61

[10] W. Arlt, B. Bungert, G. Sadowski, S. Behme, Thermisches Trennverfahren für vermischte Polymere, Patent AZ 19806 355.5-43

[11] W. Arlt, G. Sadowski, M. Seifert, S. Behme, Verfahren zur Trennung vermischter Polyolefine, Patent AZ 19905029.5

[12] N. T. Theyssen, Z. H. Zou, W. W. Wiesenhöfer, G. F. Francio, W. L. Leitner, J. Panten, Chem. Ing. Tech. 75 (2003) 1154

[13] P. G. Debenedetti, J. Supercritical Fluids 7 (1994) 9

[14] H. C. M. van Boxtel, Dissertation, Göttingen (2000)

[15] T. Dröge, Dissertation, Göttingen (1997)

[16] M. Lora, F. Rindfleisch, M. A. McHugh, J. Appl. Polym Sci 73 (1999) 1979

[17] H.-S. Byun, B. M. Hasch, M. A. McHugh, F.-O. Mähling, M. Busch, M. Buback, Macromolecules 29 (1996) 1625

[18] M. Buback, H. Latz, Macromol. Chem. Phys. 204 No.4 (2003) 638

[19] J. Groß, Dissertation, Berlin (2001) 


\section{Materialien und Methoden}

\subsection{Studium von Copolymerisationsreaktionen}

\subsubsection{Verwendete Chemikalien}

Die zur Copolymersynthese verwendeten Chemikalien sind mit dem vom Hersteller angegebenen Reinheitsgrad in Tabelle 3.1 aufgeführt. Propylacrylat wurde nach einer Vorschrift von Rehberg [1] synthetisiert und mit Hydrochinonmonomethylether stabilisiert. Ethen wurde innerhalb der Anlage zur Copolymersynthese weiter aufgereinigt (vgl. Abschnitt 3.1.2). Sämtliche (Meth)Acrylsäureester wurden unmittelbar vor der Copolymerisation mittels eines Degassers (ERC3415 Degasser, ERC, Altegolfsheim (Regensburg)) entgast (vgl. Abb. 3.1). Der für einzelne Copolymerisationen verwendete Initiator Di-tert-butylperoxid (gelöst in $n$-Heptan) wurde durch Umgasen mit Stickstoff von Sauerstoff befreit.

\begin{tabular}{|c|c|c|}
\hline Chemikalie & Reinheitsgrad & Hersteller \\
\hline Ethen & $99.8 \%$ & Linde $A G$ \\
\hline Ethylacrylat $^{(1)}$ & $99.5 \%$ & Fluka AG \\
\hline Propylacrylat $^{(1)}$ & n. best. & Synthese nach [1] \\
\hline Methacrylsäuremethylester ${ }^{(1)}$ & $99.0 \%$ & Fluka AG \\
\hline Methacrylsäureethylester ${ }^{(2)}$ & $99 \%$ & Fluka AG \\
\hline Methacrylsäure- $n$-butylester ${ }^{(3)}$ & $99 \%$ & Fluka AG \\
\hline Methacrylsäurebenzylester ${ }^{(3)}$ & $96 \%$ & Aldrich \\
\hline Methacrylsäure ${ }^{(4)}$ & $99 \%$ & Aldrich \\
\hline$n$-Heptan & mind. $99 \%$ & Merck-Schuchardt \\
\hline Di-tert-butylperoxid & mind. $98 \%$ & Merck-Schuchardt \\
\hline
\end{tabular}

Tabelle 3.1: $\quad$ Verwendete Chemikalien und der vom Hersteller angegebene Reinheitsgrad (1) stabilisiert mit $0.0025 \%$ Hydrochinonmonomethylether

(2) stabilisiert mit $0.0015 \%$ Hydrochinon

(3) stabilisiert mit $0.005 \%$ Hydrochinonmonomethylether

(4) stabilisiert mit $0.025 \%$ Hydrochinonmonomethylether 


\subsubsection{Experimentelle Anordnung zur kontinuierlichen Hochdruck- Copolymerisation}

Die im Rahmen der vorliegenden Arbeit durchgeführten Copolymerisationsexperimente wurden bei kontinuierlicher Reaktionsführung mit einer von Busch [2] entwickelten und weiterhin in Arbeiten von Buback et al. [3, 4], Lovis [5] und Wittkowski [6] beschriebenen Mini-Technikumsanlage zur radikalischen Hochdruck-Polymerisation ausgeführt. In diesem Abschnitt wird eine Übersicht des Aufbaus des Mini-Technikums gegeben und es werden einzelne Bauteile näher erläutert.

Um eine gleichbleibend hochreine Qualität des eingesetzten Ethens sicherzustellen, wird das Gas innerhalb der Anlage aufgereinigt. Hierzu sind in das Strömungssystem unmittelbar vor der ersten Kompressionsstufe zwei zylindrische Druckbehälter mit einem Volumen von jeweils 11.71 eingebaut. Diese bis zu einem Druck von 50 bar TÜV-zugelassenen Autoklaven bestehen aus je einem Edelstahlrohr von 1000 mm Länge. Die Rohre haben einen Außendurchmesser von $141.3 \mathrm{~mm}$ und sind mit aufgeschraubten Flanschen (200 x $30 \mathrm{~mm})$ versehen. Die Wandstärke der Behälter beträgt $9.5 \mathrm{~mm}$. Die stirnseitigen Deckel $(200 \mathrm{x}$ $30 \mathrm{~mm}$ ) sind mit den Behältern über jeweils 12 Bolzen (M 12) verschraubt, wobei zur Dichtung O-Ringe aus Viton ${ }^{\circledR}$ (DuPont) verwendet werden. Um den Austrag der Gasreinigungsschüttungen zu verhindern sind vor den Auslassbohrungen am Boden der Behälter Sinterfilter angebracht. Der zuerst von Ethen durchströmte Autoklav ist mit einem Katalysator auf Kupfer/Kupferoxidbasis gefüllt (BASF, R3-15), an dem Sauerstoffspuren adsorbiert werden. Im zweiten, mit Molsieb (BASF, EPG 3A) beschickten Druckbehälter werden Wasserspuren entfernt ${ }^{1}$. Der Katalysator muss vor der eigentlichen Verwendung als auch nach erfolgter Sättigung bei einer Temperatur von 200 bis $250^{\circ} \mathrm{C}$ mit Wasserstoff reduziert werden. Das Molsieb wird anschließend bei möglichst hohen Temperaturen (ca. $200^{\circ} \mathrm{C}$ ) und vermindertem Druck getrocknet. Für die Aktivierung der Schüttungen sind die beiden Behälter jeweils mit einem in einer Messingmatrix eingelassenen Widerstandsheizleiter ausgerüstet.

\footnotetext{
${ }^{1}$ Im Rahmen der experimentellen Arbeiten hat sich gezeigt, dass die Schüttungen zur Gasreinigung den Wartungsbedarf des eingesetzten Massenflussreglers erheblich reduzieren. Vor Einbau der Autoklaven in das Strömungssystem wurde ein häufiger Ausfall der Reglereinheit durch Ölablagerungen beobachtet. Dies lässt sich darauf zurückführen, dass die vermutlich in Spuren auch in hochreinem Ethen vorhandenen Öle während des Expansionsvorgangs in der Drossel des Reglers akkumulieren. Derartige Öle sollten in den Reinigungsschüttungen zurückgehalten werden.
} 
In Abbildung 3.1 ist der Gesamtplan des Minitechnikums schematisch dargestellt. Kommerzielles Ethen des Reinheitsgrades 2.8 aus einer Druckgasflaschenbatterie (F1-F5) wird durch ein auf $28^{\circ} \mathrm{C}$ geheiztes Reduzierventil vom Flaschendruck auf 10 bar entspannt.

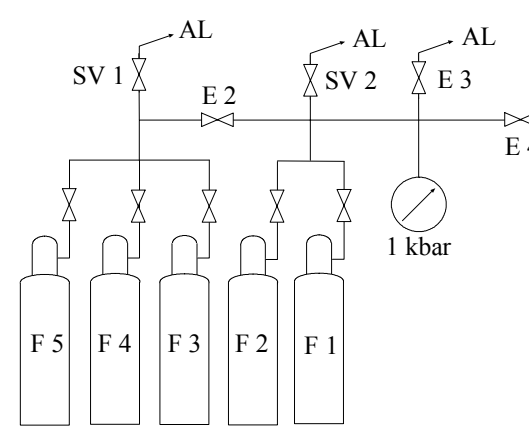

Ethen-Vorrat

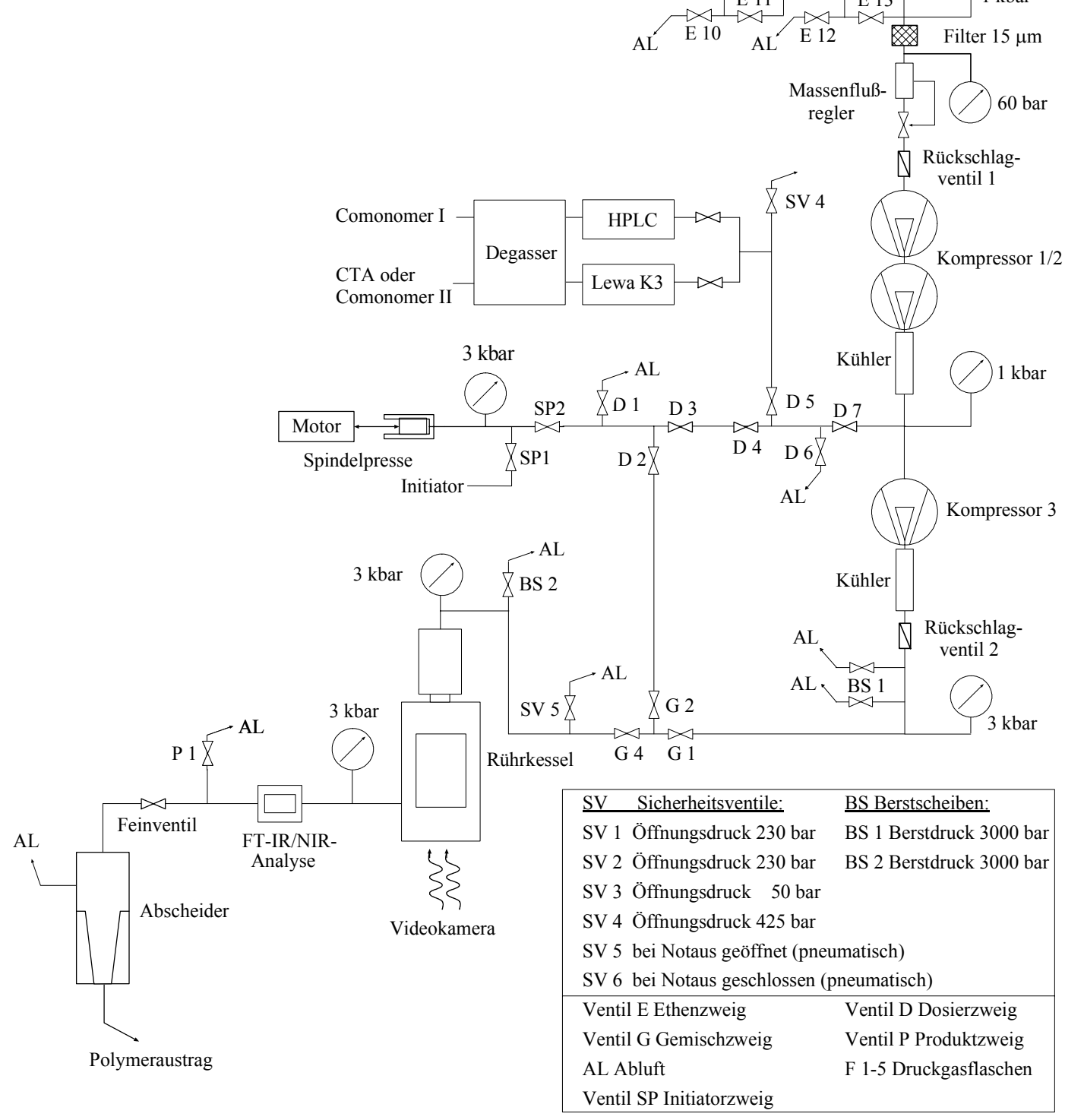

Abbildung 3.1.: Schematische Darstellung der Anlage zur Hochdruck-Copolymerisation. 
Nach Durchströmen der Aufreinigungseinheit (s.o.) hält ein nachgeschalteter Hochdrucksinterfilter (Porenweite $15 \mu \mathrm{m}$, Swagelock 10-TF) eventuelle Staubreste aus der Katalysator- bzw. Molsiebschüttung zurück.

Das nunmehr hochreine Ethen $\left(\mathrm{O}_{2}\right.$-Gehalt $<0.1 \mathrm{ppm}$, nach Angabe des Katalysatorherstellers) strömt durch einen Massenflussregler (5851E, Brooks-Instrument, Mannheim), welcher den Massenfluss misst und entsprechend einer externen Sollwertvorgabe regelt. Anschließend wird das Ethen von einem zweistufigen Membrankompressor (Hofer, Mühlheim / Ruhr) auf ca. 250 bar verdichtet. Durch einen einstufigen Nachverdichter (ebenfalls Hofer) wird der für alle Synthesen gewählte Druck von 2000 bar erreicht. Vor dieser dritten Kompressionsstufe können mittels einer computergesteuerten Kolbenmembranpumpe (Typ K3, Lewa) und / oder einer HPLC-Pumpe (Typ Maxi-Star 1000, Knauer) Comonomere, Molmassenregler und Initiatorlösung in die Saugseite des Nachverdichters gefördert werden. Zum Entfernen der in den Initiatorlösungen, Comonomeren und Molmassenreglern gelösten Gase, wird der Saugstrom der Dosierpumpen durch eine Degasser (s. Abschnitt 3.1.1) geleitet. Alternativ kann der Initiator auch mittels einer durch Schrittmotoren gesteuerten Spindelpresse [7] sowohl vor der dritten Kompressorstufe bei etwa 250 bar oder nach derselben bei dem gewählten Reaktionsdruck eindosiert werden. ${ }^{2}$ Die so hergestellte Comonomermischung gelangt anschließend über 1/4-Zoll-Hochdruckkapillaren in den Hochdruck-Hochtemperatur-Rührautoklaven, in dem die Polymerisation stattfindet.

Die Eindosierung der Comonomermischung in den Rührkessel wurde im Unterschied zu vorangegangenen Arbeiten [2,7,6] derart abgeändert, dass anstelle des seitlichen Einströmens die Zufuhr nunmehr über den Rührantrieb in den Rührkessel erfolgt. Hierdurch wird vermieden, dass bei Druckschwankungen Reaktionsprodukte in den Innenraum des Rührantriebs eingespült werden.

Die Homogenität der Reaktionsmischung kann durch ein auf der Unterseite des Reaktors eingelassenes Saphirfenster visuell über eine Videokamera mit daran angeschlossenem Monitor kontrolliert werden. Nach Verlassen des Rührkessels strömt die nun auch Copolymer enthaltende Mischung über geheizte Hochdruckkapillaren durch eine optische Hochdruckzelle [32], die im Strahlengang eines FT-IR/NIR-Spektrometers (IFS 88, Bruker) fixiert ist. In dieser werden zur Kontrolle des stationären Zustands der Synthese Umsatz und Gemischzusammensetzung durch ,inline“-FT-IR/NIR-Spektroskopie beobachtet.

\footnotetext{
${ }^{2}$ Trotz der im Vergleich längeren Wartezeit auf stabile Betriebsbedingungen hat es sich als vorteilhaft erwiesen den Initiator vor der Kompressionsstufe einzudosieren. Hierbei ergibt sich durch den stabilen Vordruck der dritten Stufe leichter ein schwankungsfreier Initiatordosierstrom.
} 
Die Temperatur der Produkt-Edukt-Mischung in den Hochdruckkomponenten nach dem Rührkessel wird mindestens $25^{\circ} \mathrm{C}$ geringer als die Reaktortemperatur aber noch so hoch, gewählt, dass eine Phasentrennung vermieden wird. Aufgrund der kurzen Verweilzeit in diesem Teil der Anlage kann eine Weiterreaktion vernachlässigt werden. Durch anschließendes Entspannen der Reaktionsmischung an einem speziellen Feinventil [3] wird das Polymer in einem Normaldruckabscheider [6] von den gasförmigen Bestandteilen separiert und quantitativ in mit Schlitzen zur Gasableitung versehenen Schraubdeckelflaschen aus Polyethylen aufgefangen.

Die Temperaturen im Reaktor, in der optischen Hochdruckzelle, an den Kapillaren und Ventilen werden mit Mantelthermoelementen (Nickel-Chrom gegen Nickel-Chrom-Alumel, CIA S250, CGE Alsthom) gemessen. Im Reaktor sind dazu in drei Positionen Messstellen vorgesehen (s.u.), so dass eventuell auftretende Temperaturgradienten leicht erkannt werden können. Der Reaktor, die Feinventile und die IR-Hochdruckzzelle werden elektrisch mit Mantelheizleitern (Pyrolon-M. $16 \Omega \mathrm{m}^{-1}$, Les Cables de Lyon) beheizt, die in jeweils über die Bauteile geschobenen Messingmatrizen eingelassen sind. Für die Temperaturregelkreise werden PID-Regler (Eurotherm, Typ 19, Typ 815 und Typ 2116) eingesetzt. Im Hochdruckteil der Anlage werden die Drücke mit DMS-Druck-Umwandlern (HBM-Messtechnik, Klasse 0.2, Maximaldruck $3 \mathrm{kbar}$ ) an folgenden Positionen gemessen: nach dem Nachverdichter, am Eingang des Rührantriebs und vor der IR-Zelle.

Um Gefahren durch versagende Hochdruckbauteile abzuwenden, sind alle Anlagekomponenten mit Überdruckventilen bzw. Berstscheiben ausgestattet (siehe Abb. 3.1). Darüber hinaus sind in und außerhalb der Druckschutzkabine Notausschalter angebracht, mit denen über elektropneumatisch gesteuerte Ventile gleichzeitig die Ethenversorgung unterbrochen und die Anlage in die Abluft druckentlastet werden kann (s. Abb. 3.1).

\section{Hochdruck-Rührkessel}

Der Hochdruck-Rührkessel stellt das Kernstück der Minitechnikumsanlage dar. Im folgenden werden die wesentlichen Baugruppen beschrieben. Detaillierte Informationen über die Konstruktion und Entwicklung des Autoklaven finden sich bei Busch [2], Buback et al. [3] und Lovis [5]. In Abbildung 3.2 ist der Hochdruck-Hochtemperatur-Autoklav dargestellt. Der Autoklav besitzt unter Berücksichtigung aller Einbauten ein Innenvolumen von $51.5 \mathrm{~cm}^{3}$. Er besteht aus einem zylindrischen Hohlkörper von $170 \mathrm{~mm}$ Länge und besitzt einen Außendurchmesser von $150 \mathrm{~mm}$. Der Innendurchmesser beträgt $42 \mathrm{~mm}$. 


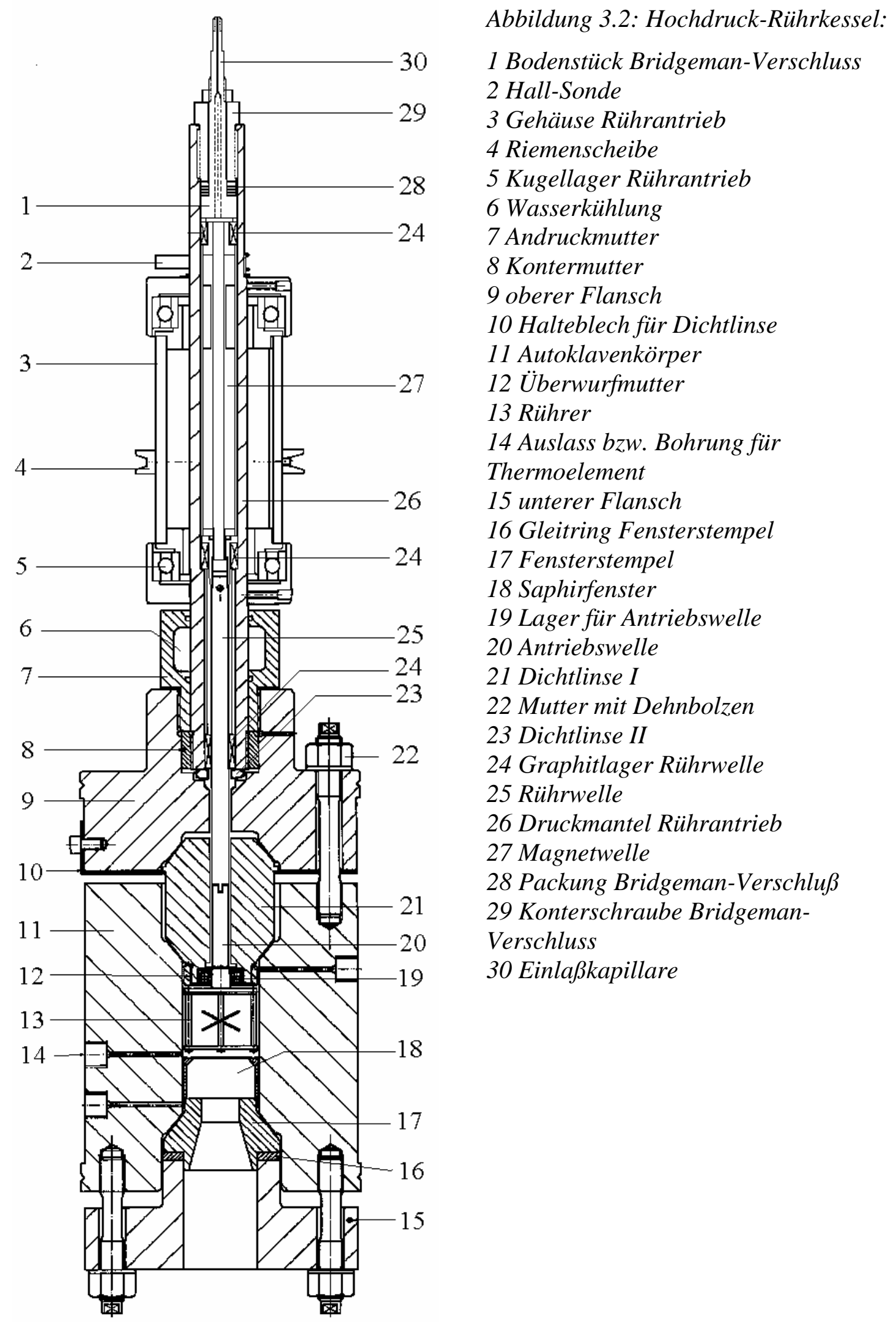


Der Körper des Autoklaven sowie der obere und untere Flansch sind aus einer hochwarmfesten Nickelbasislegierung (RGT 601, Werkstoff-Nr. 2.4668, Arbed-Saarstahl) gefertigt. Das Verhältnis von Innen- zu Außendurchmesser erlaubt unter Berücksichtigung eines Sicherheitsbeiwertes von 2.5 in Verbindung mit dem gewählten Material eine maximale Druckbelastung von 3000 bar bei $300^{\circ} \mathrm{C}$.

Die Unterseite des Reaktors wird durch einen Stempel (RGT 12, Werkstoff-Nr. 2.4969, Arbed-Saarstahl) mittels einer Metall-Metall-Konusdichtung verschlossen. Der Stempel wird durch den unteren Flansch mit zwölf Dehnbolzen mit einem Drehmoment von jeweils $90 \mathrm{Nm}$ auf den Autoklaven-Hauptkörper gepresst. Der Einsatz von Dehnbolzen verhindert, dass die Gewindebohrungen im Reaktorblock bei hohen Drucksprüngen beschädigt werden. Der untere Dichtstempel besitzt eine zentrale Bohrung von 21.2 mm Durchmesser. Dieser Stempel trägt zur optischen Kontrolle des Reaktorinnenraums ein aus synthetischem Saphir (UV-Grade, Roditi, Union Carbide) gefertigtes Fenster. Der Durchmesser des Saphirfensters beträgt $38.1 \mathrm{~mm}$, die Höhe misst $22.4 \mathrm{~mm}$. Auf der Seite des Rührantriebs wird der Reaktor durch eine Dichtlinse (Werkstoff RGT 12) ebenfalls über Konusdichtungen abgeschlossen. Die Linse ist axial durchbohrt, um den Rührer über eine Welle mit dem Rührantrieb zu verbinden. Der Innendurchmesser der Linse beträgt $11 \mathrm{~mm}$. An der Unterseite der Linse befindet sich ein Edelstahlkugellager (SS-6000-2 ZJ), von dem zum besseren Durchgang des Eduktstromes die seitlichen Dichtungsscheiben entfernt werden. Das Lager wird mit einer Überwurfmutter in der Dichtlinse fixiert.

Der Antrieb des Rührers erfolgt über eine Hochdruck-Magnetkupplung (Typ 0,75-4-50 KMP 35N Spec., Autoclave Engineers). Um den Wärmefluss vom Reaktor zur Magnetkupplung zu verhindern, ist der Verbindungsflansch wassergekühlt. Die äußeren Permanentmagnete werden über eine Riemenscheibe von einem Syncro-Servo-Motor mit entsprechender Regelelektronik (Seidel) angetrieben. Die bis zu $1500 \mathrm{~min}^{-1}$ stufenlos einstellbaren Drehzahlen des Rührantriebs werden über eine Hallsonde kontrolliert. Als Rührkörper wurde bei den Copolymersynthesen der von Lovis [5] entwickelte Käfig-PropellerRührer verwendet. Anhand von Messungen der Verweilzeitverteilung wurde belegt, dass der Reaktor mit dem verwendeten Rührertyp und dem beschriebenen Dosierweg als annähernd ideal rückvermischter Rührkesselreaktor beschrieben werden kann [8].

Der Rührkessel besitzt 6 Bohrungen mit einem Durchmesser von jeweils $1.8 \mathrm{~mm}$, die im Winkel von $90^{\circ}$ in drei unterschiedlichen Höhen radial eingelassen sind. In jeweils einer der oberen und unteren Bohrungen wird die Temperatur der Reaktorwand gemessen. Die beiden mittleren Bohrungen sind so angeordnet, dass sie sich gerade über dem Saphirfenster, also 
direkt oberhalb des Reaktorbodens, befinden. Eine der Bohrungen dient als Auslaß der Reaktionsmischung, die andere wird zur Einführung eines Mantelthermoelements genutzt mit welchem die Temperatur der Reaktionsmischung in der Mitte des Reaktorinneren gemessen werden kann.

\subsubsection{Auswahl der Messbedingungen}

Der experimentell zugängliche Druck- und Temperaturbereich für Copolymerisationen von Ethen mit (Meth)Acrylaten ist aufgrund folgender Aspekte beschränkt:

\section{(1) Apparative Druck und Temperaturgrenze:}

Der für die Synthesen eingesetzte Hochdruckautoklav ist inklusive der Anbauteile bis zu einem maximalen Druck von 3000 bar bei Temperaturen bis $300{ }^{\circ} \mathrm{C}$ einsetzbar.

\section{(2) Phasenverhalten:}

Der zugängliche Druck- und Temperaturbereich wird zu geringen $p$ - und $T$-Werten durch die Inhomogenität der Reaktionsmischung begrenzt. Während der Synthese sollte Zweiphasigkeit vermieden werden, da ansonsten die Reaktionsführung durch verstärktes Reaktorfouling erschwert und das Risiko einer thermischen Zersetzungsreaktion des Ethens [9] erhöht wird. Zudem ist im Rahmen dieser Arbeit geplant, die Zusammensetzungskinetik der Copolymerisationen zu untersuchen. Dies gelingt in der Regel nur für Systeme in homogener Phase, da in mehrphasigen Systemen die Monomere und Polymere in schwer messbarem Umfang in den verschiedenen Phasen vorliegen.

Literaturdaten von z.B. McHugh et al. [40] für die Systeme Ethen / Poly(Ethen-coMethylacrylat) (E/E-MA) und Ethen / Poly(Ethen-co-Butylacrylat) (E/E-BA) belegen, dass im Zusammensetzungsbereich der Copolymere von ca. 0 - 50 mol-\% bei Synthesetemperaturen von $150^{\circ} \mathrm{C}$ und höher für eine homogene Reaktionsführung Reaktionsdrücke von 1800 bar ausreichend sind. Dröge [10] hat die Druckabhängigkeit der Copolymerisationsparameter für die verwandten Copolymersysteme Ethen-Methylacrylat, Ethen-2Ethylhexylacrylat und Ethen-Butylmethacrylat gemessen. Es zeigte sich, dass die Druckabhängigkeit im untersuchten Bereich von 1500 - 2500 bar sehr gering ist, wobei die experimentelle Unsicherheit im Verhältnis zur Größenordung des Effekts groß ist. Es wurde daher für alle im Rahmen dieser Arbeit ausgeführten Copolymersynthesen ein Synthesedruck von 2000 bar gewählt und auf die Untersuchung der Druckabhängigkeit verzichtet. An dieser Stelle sei erwähnt, dass bei allen im Rahmen der vorliegenden Arbeit durch- 
geführten Copolymersynthesen Einphasigkeit der Reaktionsmischung herrschte. Dies ist durch eine visuelle Überwachung der Reaktionsmasse im Reaktor (s. Abschnitt 3.1.2) geprüft worden ${ }^{3}$.

\section{(3) Initiierungskinetik:}

Bei den Copolymersynthesen wurde darauf abgezielt, den jeweiligen Gesamtumsatz möglichst gering zu halten. Der überwiegende Teil der Experimente wurde daher ohne Einsatz von Initiatoren durchgeführt. Dies ist möglich, da bei der Hochdruckpolymerisation von Ethen sowie bei allen bisher untersuchten Ethen-(Meth)Acrylat-Copolymerisationen bei Temperaturen oberhalb von ca. $165^{\circ} \mathrm{C}$ eine Selbstinitiierung auftritt $[10,29]$. Der Umfang dieser Selbstinitiierung nimmt mit der Temperatur und der (Meth)Acrylatkonzentration zu. Entsprechend findet die Synthese von Ethen-(Meth)Acrylat-Copolymeren bei höheren Synthesetemperaturen dort eine Beschränkung, wo die Selbstinitiierung der Monomermischung einen Umsatz größer als die im Rahmen dieser Arbeit gewählte Obergrenze von $10 \%$ bewirkt. Der maximale Comonomergehalt der hergestellten Copolymerproben wird somit bei hohen Synthesetemperaturen durch die Initiierungskinetik begrenzt. Bei niedrigeren Temperaturen beschränkt die Auslegung der dritten Kompressionsstufe (flüssiger Anteil im Dosierstrom $\leq 10$ Vol.-\%) die Synthese von Ethen-(Meth)Acrylat-Copolymeren auf Produkte mit weniger als 60 mol-\% (Meth)Acrylat.

Der Vorteil niedriger Umsätze liegt darin, dass unter diesen Bedingungen Copolymermaterial erhalten wird, welches eine eher schmalere Molekulargewichtsverteilung sowie eine bestmögliche chemische Einheitlichkeit (d.h. eine nahezu gleichartige Zusammensetzungsverteilung in den Copolymermolekülen) aufweist. Derartige Proben sind für experimentelle Trübungsdruckmessungen besonders geeignet, da die Präzision von Trübungsdruckmessungen mit schmaler werdender Molekulargewichtsverteilung steigt. Darüber hinaus - und dies gilt insbesondere vor dem Hintergrund der in dieser Arbeit vorgesehenen modellierenden Beschreibung der Ergebnisse der Trübungsdruckmessungen (vgl. Abschnitt 6) - ist es sinnvoll mögliche Unterschiede in der Einheitlichkeit der untersuchten Copolymere, welche prinzipiell einen Einfluss auf das Entmischungsverhalten haben können [38], zu vermeiden.

\footnotetext{
${ }^{3}$ Das Auftreten von Inhomogenität wäre neben der optischen Überwachung zudem auch bei der inlineNIR/IR-spektroskopischen Kontrolle der Reaktionsmischung in Form einer zunehmenden Basislinie aufgefallen.
} 


\subsubsection{Durchführung von Copolymerisationsexperimenten}

\section{Vorbereitung eines Syntheseexperiments}

Vor jedem Experiment werden der Reaktor und alle nachfolgenden Komponenten der Anlage zerlegt und gründlich gereinigt. Hierzu werden eventuelle Copolymeranhaftungen nach Aufweichen bei Temperaturen bis $180^{\circ} \mathrm{C}$ in flüssigem Isododekan mechanisch entfernt und Lösungsmittelreste mit Aceton abgespült. Copolymerreste in zugesetzten Hochdruckbauteilen und Kapillaren werden ebenfalls auf bis $\mathrm{zu} 300^{\circ} \mathrm{C}$ erhitzt und mit komprimiertem Kohlendioxid oder Heptan ausgetragen. Spätestens am Vortag des Experiments wird die Anlage mehrfach mit Ethen gespült und mindestens 12 Stunden unter einem Ethendruck von 2000 bar belassen. Dies dient zum einen als Test der Dichtigkeit, zum anderen können sich während dieser Zeitspanne Sauerstoffspuren und andere Verunreinigungen im überkritischen Ethen lösen und anschließend mit diesem ausgetragen werden.

\section{Durchführung eines Syntheseexperiments}

Unmittelbar vor dem Versuch wird der Druck in der Anlage abgelassen. Der Dosierzweig für Comonomere, Molmassenregler und Initiatoren wird mit den jeweiligen Substanzen gespült. Der Kühlwasserkreislauf der Kompressoren und des Rührantriebs wird geöffnet. Alle heizbaren Komponenten werden auf die jeweils gewählte Temperatur gebracht. Sobald die Analysenzelle ihre Solltemperatur erreicht hat, wird ein Referenzspektrum der Zelle aufgenommen. Nun wird nach dem Start der Comonomerdosierung bei geöffnetem Ventil des Massenflussreglers in den Kapillaren bis unmittelbar vor dem Reaktor Druck aufgebaut. Sobald der gewünschte Reaktionsdruck erreicht ist, wird das Absperrventil G4 zwischen Reaktor und der dritten Kompressionsstufe (vgl. Abb. 3.1) geöffnet und der Rührmotor eingeschaltet. Anschließend wird der Ethenfluss mit Hilfe des Massenflussreglers eingeregelt. Der Reaktionsdruck wird manuell mit Hilfe des Feinventils reguliert. Der gewählte Massenfluß der Meth)Acrylate beträgt bei dem für alle Experimente eingestellten Ethendurchsatz von $1 \mathrm{~kg} / \mathrm{h}$ zwischen 4 und $260 \mathrm{~g} / \mathrm{h}$.

Während des Betriebs registriert das FT-IR-Spektrometer automatisch Spektren in einem Zeitabstand von 2 Minuten, wobei für jedes Absorbanzspektrum 64 Interferogramme zwischen 1400 und $6500 \mathrm{~cm}^{-1}$ innerhalb von 25 Sekunden aufgenommen werden. Die Einkanalspektren werden nach Fouriertransformation der Interferogramme unter Anwendung einer Blackmann-Harris 3-Term Apodisierungsfunktion berechnet. Für die Spektrenaufnahme finden folgende optische Komponenten Anwendung: Globar-Lichtquelle, 
Siliciumbeschichteter Calciumfluorid-Strahlenteiler und ein mit flüssigem Stickstoff gekühlter MCT-Detektor. Anhand der IR-Spektren lässt sich die Änderung der (Meth)Acrylat-Konzentration sowie die Änderung der Konzentration des entstehenden Copolymers verfolgen [10]. Nach Erreichen stationärer Betriebsbedingungen, erkennbar an der Konstanz der aufgenommenen IR-Spektrenserie, wird mindestens 5 Verweilzeiten abgewartet (ca. 15 min.). Hiernach wird innerhalb eines definierten Zeitraums eine Polymerprobe in der Abscheideeinheit [6] genommen. Anschließend kann der Comonomerfluss oder die Reaktortemperatur variiert und eine neue Synthesebedingung angefahren werden.

Im Unterschied zur Ethen-(Meth)Acrylat-Copolymerisation bei der durch den thermischen Umsatz (s.o.) keine zusätzliche Initiierung erforderlich ist, muss bei der Copolymerisation von Ethen und Methacrylsäure ein Initiator zur Erzielung von Umsatz eingesetzt werden. Der Initiatorverbrauch hängt aus bisher nicht geklärten Gründen erheblich von der Konzentration des Comonomers Methacrylsäure in der Reaktionsmischung ab. Auch bei den im Rahmen dieser Arbeit durchgeführten Ethen-Methacrylsäure-ButylmethacrylatTerpolymerisationen zeigte sich eine Verknüpfung des Umsatzes mit dem Methacrylsäuredosierstrom bei konstanter Dosierung des eingesetzten Initiators (DtBP). Um die Synthesen bei gleichem Umsatz auszuführen, muss daher mit zunehmender Säureförderung auch die Initiatordosierung gesteigert werden. Aufgrund der starken Wechselwirkung von Methacrylsäurekonzentration, Initiatorverbrauch und Umsatz können Schwankungen in der Initiatoroder Säuredosierung (wie z.B. durch kurzeitigen Ausfall der Dosierpumpe) schnell zum Verlust der Kontrolle über die Reaktion führen. Entsprechend ist bei diesen Copolymerisationen eine besonders sorgfältige Kontrolle der Synthesebedingungen mit der „,inline“-Spektroskopie erforderlich. Details hierzu finden sich bei Wittkowski [6].

\section{Beendigung eines Experiments}

Zur Beendigung der Copolymersynthese wird, falls Initiator verwendet wurde, zunächst die Initiatordosierung ausgestellt und abgewartet bis anhand der ,inline“-Spektroskopie kein weiterer Umsatzrückgang erkennbar ist. Anschließend wird die (Meth)Acrylatdosierung ausgestellt. Sobald spektroskopisch bestimmt wird, dass die (Meth)Acrylatkonzentration im Feedstrom vollständig abgesunken ist, werden die Kompressoren auf Bypass-Förderung geschaltet und die Anlage über das Feinventil druckentlastet. Die Heizungen und Kompressoren werden abgeschaltet. Die (Meth)Acrylat-Dosierpumpen und die Motorspindelpresse werden bei hohem Fluss mit einer Mischung aus Aceton und Heptan für mindestens eine Stunde gespült. 


\subsubsection{Aufarbeitung der Copolymerproben}

Für die weitere Auswertung werden die erhaltenen Produkte zwecks Entfernung der Restmonomere bei Zimmertemperatur und einem Druck von $\leq 10^{-3}$ mbar bis zu 14 Tage getrocknet. Der Fortgang der Trocknung wird gravimetrisch kontrolliert. Im Falle schwerflüchtiger Comonomere (z.B. Benzylmethacrylat) ist es sinnvoll, die Proben am Ende des Trocknungsprozesses auf Temperaturen bis max. $60^{\circ} \mathrm{C} \mathrm{zu}$ erhitzen. Ein Erwärmen von Ethen-Acrylat-Copolymeren bereits zu Trockungsbeginn sollte vermieden werden, da sich anhand von GPC-Ergebnissen gezeigt hat, dass diese Materialien unter diesen Bedingungen (Anwesenheit einer höheren Monomerkonzentration) z.T. vernetzen [8]. Die Vollständigkeit der Monomerabtrennung wird NMR- und / oder IR-spektroskopisch geprüft (s. a. Abschnitt 3.2.1). Die gereinigten Polymerproben werden zur Bestimmung des Trocknungsverlustes mit einer Analysenwaage (Sartorius Typ AC 210 S) ausgewogen.

Für die Trübungsdruckmessungen der Randsysteme wurden ergänzend Homopolymere synthetisiert. Eine Syntheseanleitung sowie eine Aufstellung der hergestellten Proben findet sich in Anhang B dieser Arbeit. 


\subsection{Copolymeranalyse}

\subsubsection{Bestimmung der Copolymerzusammensetzung mittels ${ }^{1}$ H-NMR- Spektroskopie}

Die Zusammensetzung der synthetisierten Copolymerproben wurde mittels ${ }^{1} \mathrm{H}-\mathrm{NMR}$ Spektroskopie analysiert. Der Vorteil dieser Methode gegenüber der Bestimmung durch Elementaranalyse als Alternative liegt darin, dass Verunreinigungen durch Monomerreste oder Wasserspuren leicht festzustellen als auch quantitativ bestimmbar sind. Darüber hinaus können Hinweise auf möglicherweise während der Synthese oder Aufarbeitung auftretende Nebenreaktionen, wie z.B. Verseifung der Esterfunktionen, erhalten werden.

Die Fläche unter einem Kernresonanzsignal ist direkt proportional zu der Zahl der bei dieser Resonanzfrequenz des Spektrums absorbierenden Kerne eines Moleküls. Entsprechend kann die absolute Zusammensetzung eines aus zwei oder mehreren verschiedenen Comonomeren gebildeten Copolymeren bestimmt werden, wenn die Signale der jeweiligen Molekülbausteine getrennt detektierbar sind.

In den Abbildungen 3.3 und 3.4 ist exemplarisch für die verschiedenen Copolymersysteme jeweils ein ${ }^{1}$ H-NMR-Spektrum dargestellt. Die Zuordnung der Signale zu den Protonen der jeweiligen Strukturen in den Copolymeren ist in die Spektren eingezeichnet.

Die Peaks der $\alpha-\mathrm{CH}_{\mathrm{X}}$-Gruppen (x=2 bzw. 3) der Esterseitenkette finden sich isoliert bei etwa 3.7 ppm (Ethen-Methylmethacrylat-Copolymer) bzw. bei 4.1 ppm (Copolymere des Ethens mit Ethyl(meth)- bzw. Propylacrylat bzw. Butylmethacrylat) bzw. bei 5.2 ppm (Ethen-Benzylmethacrylat-Copolymer).

Im Bereich der aliphatischen Protonen zwischen 0.9-2.3 ppm werden die Signale der Protonen der Hauptkette des Polymers, der $\alpha$-Methylgruppen und der weiter entfernten Methylen-Protonen der Estergruppe als auch diejenigen der terminalen Methylgruppen (bei Ethyl-, Propyl- und Butylmethacrylat-Copolymeren) beobachtet. Das Spektrum ist in diesem Bereich nicht vollständig aufgelöst, sodass das entsprechende Integral nur als Summe der einzelnen Beiträge ausgewertet werden kann. 


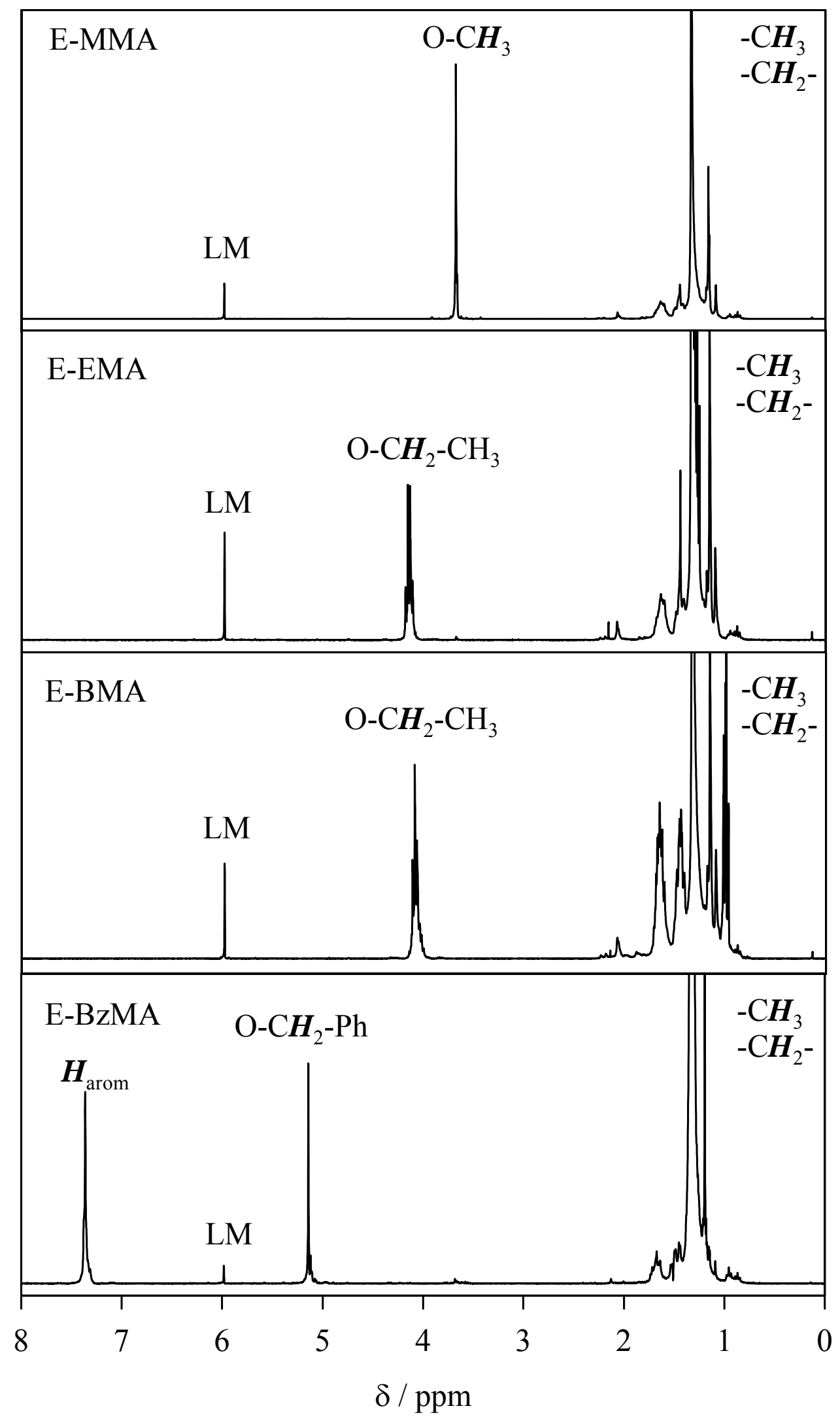

Abbildung 3.3: ${ }^{1}$ H-NMR-Spektren verschiedener Ethen-Methacrylat-Copolymere. 


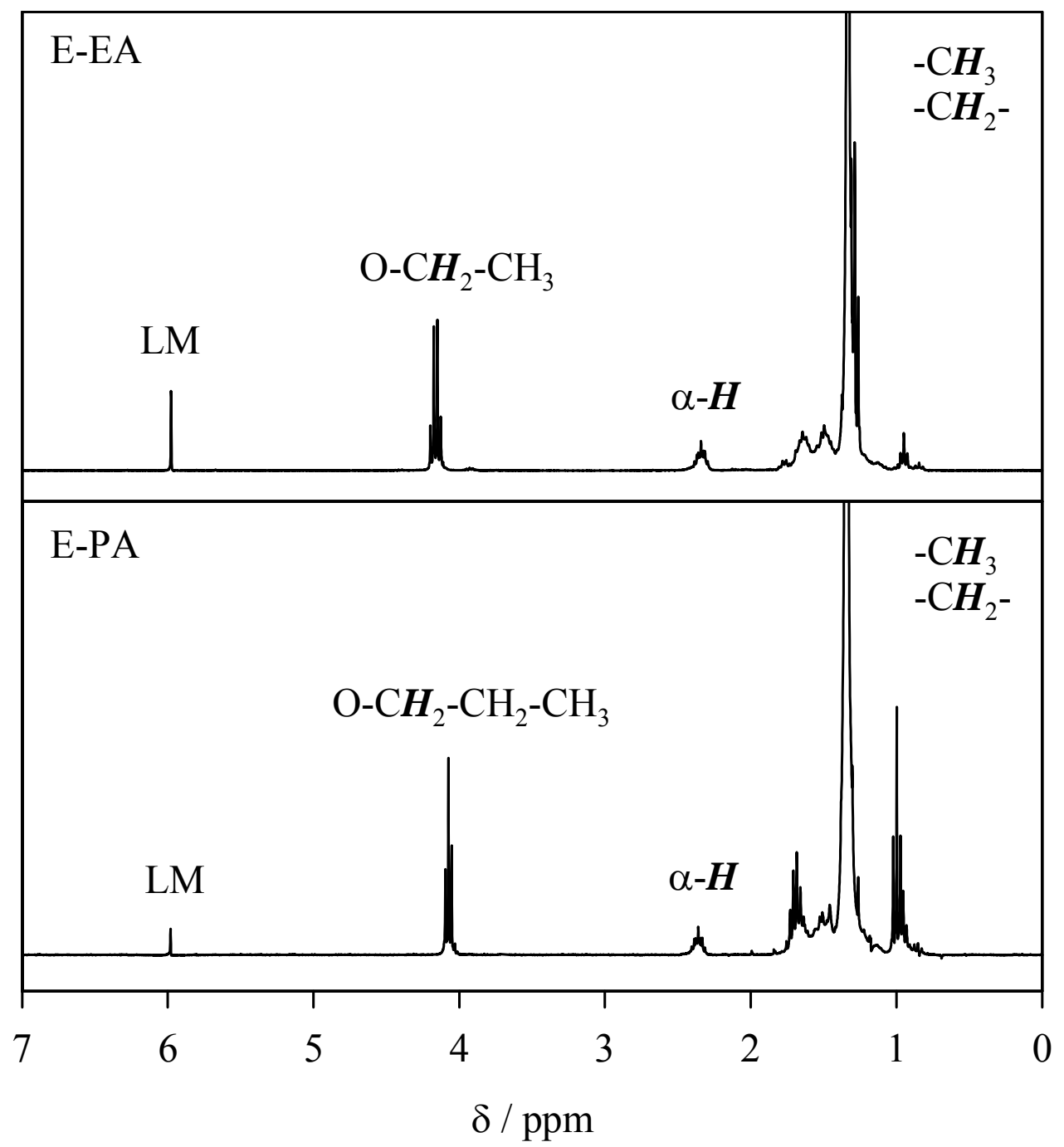

Abbildung 3.4: ${ }^{1}$ H-NMR-Spektrum eines Ethen-Ethylacrylat-Copolymers (oben) bzw. eines Ethen-Propylacrylat-Copolymers (unten).

In den NMR-Spektren der Ethen-Benzylmethacrylat-Copolymere wird im Unterschied zu denen der anderen Systeme bei etwa $7.4 \mathrm{ppm}$ zusätzlich das Signal der aromatischen Protonen beobachtet. Dieser Peak tritt wie jener der O-CH2-Gruppen (vgl. Methode A, Gl. 3.5) isoliert auf, so dass man das Integral dieses Peaks ebenfalls zur Berechnung der Zusammensetzung für dieses Copolymersystem verwenden kann (Methode B, Gl. 3.6). Die nach den beiden Berechnungsmethoden erhaltenen Zusammensetzungen der E-BzMACopolymere stimmen innerhalb der experimentellen Unsicherheit überein. Für EthenEthylacrylat- und Ethen-Propylacrylat-Copolymere tritt neben den zuvor beschriebenen Signalen noch bei etwa $2.3 \mathrm{ppm}$ die Absorbanz der Protonen auf, welche am tertiären Kohlenstoffatom der Acrylatgruppe gebunden sind $(\boldsymbol{\alpha}-\boldsymbol{H})$. Aus der Literatur [11] ist bekannt, dass in Abhängigkeit von den Reaktionsbedingungen ein Teil dieser Protonen 
während der Polymerisation durch Seitengruppen ersetzt wird. Entsprechend ist dieses Signal zur Auswertung des Gesamtcomonomergehalts ungeeignet.

Bei etwa 6 ppm findet man das Signal des Lösungsmittels (1,1,2,2-Tetrachlorethan- $\mathrm{d}_{2}$, mit „LM“ bezeichnet). Die für die Auswertung verwendeten Integrationsgrenzen der einzelnen Signale bzw. Signalgruppen sind in Tabelle 3.2 zusammengefasst.

\begin{tabular}{|c|c|c|c|}
\hline Comonomer & Strukturelement & $\begin{array}{l}\text { Integrationsgrenzen } \\
{[\mathrm{ppm}]}\end{array}$ & Bezeichnung \\
\hline \multirow{2}{*}{ Methylmethacrylat } & $\mathrm{O}-\mathrm{CH}_{3}$ & $4.00-3.30$ & $\mathrm{~S} 1$ \\
\hline & Hauptkette & $2.30-0.70$ & $\mathrm{~S} 2$ \\
\hline \multirow{2}{*}{ Ethylmethacrylat } & $\mathrm{O}-\mathrm{CH}_{2}-\mathrm{CH}_{3}$ & $4.40-3.80$ & $\mathrm{~S} 1$ \\
\hline & $\begin{array}{l}\text { Hauptkette / } \\
\mathrm{O}-\mathrm{CH}_{2}-\mathrm{CH}_{3} \\
\end{array}$ & $2.35-0.70$ & $\mathrm{~S} 2$ \\
\hline \multirow{2}{*}{ Butylmethacrylat } & $\mathrm{O}-\mathrm{CH}_{2} \mathrm{CH}_{2} \mathrm{CH}_{2}-\mathrm{CH}_{3}$ & $4.40-3.70$ & $\mathrm{~S} 1$ \\
\hline & $\begin{array}{c}\text { Hauptkette / } \\
\mathrm{O}-\mathrm{CH}_{2} \mathrm{CH}_{2} \mathrm{CH}_{2}-\mathrm{CH}_{3} \\
\end{array}$ & $2.40-0.70$ & $\mathrm{~S} 2$ \\
\hline \multirow{3}{*}{ Benzylmethacrylat } & $\mathrm{O}-\mathrm{CH}_{2}-\mathrm{C}_{6} \boldsymbol{H}_{5}$ & $7.30-7.55$ & $\mathrm{~S} 1$ \\
\hline & $\mathrm{O}-\mathrm{CH}_{2}-\mathrm{C}_{6} \mathrm{H}_{5}$ & $5.25-4.90$ & $\mathrm{~S} 2$ \\
\hline & Hauptkette & $2.40-0.70$ & $\mathrm{~S} 3$ \\
\hline \multirow{3}{*}{ Ethylacrylat } & $\mathrm{O}-\mathrm{CH}_{2}-\mathrm{CH}_{3}$ & $4.45-3.80$ & $\mathrm{~S} 1$ \\
\hline & $\begin{array}{l}\text { Hauptkette / } \\
\mathrm{O}-\mathrm{CH}_{2}-\mathrm{CH}_{3}\end{array}$ & $2.15-0.70$ & S2 \\
\hline & $\alpha-H$ & $2.50-2.20$ & $\mathrm{~S} 3$ \\
\hline \multirow{3}{*}{ Propylacrylat } & $\mathrm{O}-\mathrm{CH}_{2} \mathrm{CH}_{2}-\mathrm{CH}_{3}$ & $4.30-3.75$ & $\mathrm{~S} 1$ \\
\hline & $\begin{array}{c}\text { Hauptkette / } \\
\mathrm{O}-\mathrm{CH}_{2} \mathrm{CH}_{2}-\mathrm{CH}_{3}\end{array}$ & $2.15-0.70$ & $\mathrm{~S} 2$ \\
\hline & $\alpha-H$ & $2.50-2.20$ & $\mathrm{~S} 3$ \\
\hline
\end{tabular}

Tabelle 3.2: $\quad$ Signalzuordnung und Integrationsgrenzen für die ${ }^{1} H$-NMR-spektroskopische Bestimmung der Zusammensetzung von Ethen-(Meth)Acrylat-Copolymeren. 
Für die quantitative Auswertung eines Spektrums wird zunächst der Gesamt-Ethen-Gehalt $n_{\mathrm{E}}$ der Polymerprobe berechnet. Unter Berücksichtigung der Zahl der Protonen, die zu einem Signal beitragen, ergibt sich für die verschiedenen Copolymersysteme:

$$
\begin{array}{ll}
n_{\mathrm{E}}(\mathrm{E}-\mathrm{MMA}) & =\frac{1}{4} \mathrm{~S} 2-\frac{5}{12} \mathrm{~S} 1 \\
n_{\mathrm{E}}(\mathrm{E}-\mathrm{EMA}) & =\frac{1}{4} \mathrm{~S} 2-\mathrm{S} 1 \\
n_{\mathrm{E}}(\mathrm{E}-\mathrm{BMA}) & =\frac{1}{4} \mathrm{~S} 2-\frac{3}{2} \mathrm{~S} 1 \\
n_{\mathrm{E}}(\mathrm{E}-\mathrm{BzMA}) & =\frac{1}{4} \mathrm{~S} 3-\frac{5}{8} \mathrm{~S} 2 \\
n_{\mathrm{E}}(\mathrm{E}-\mathrm{EA}) & =\frac{1}{4} \mathrm{~S} 2-\frac{5}{8} \mathrm{~S} 1 \\
n_{\mathrm{E}}(\mathrm{E}-\mathrm{PA}) & =\frac{1}{4} \mathrm{~S} 2-\frac{7}{8} \mathrm{~S} 1
\end{array}
$$

Die Polymerkonzentration der Probelösung ist implizit in $n_{\mathrm{E}}$ enthalten, so dass keine weitere Referenzierung nötig ist. Man erhält für die (Meth)Acrylatgehalte $F_{\mathrm{A}}$ [100 mol-\%]:

$$
\begin{array}{ll}
F_{\mathrm{A}}=\frac{\mathrm{S} 1}{\frac{3}{4} \mathrm{~S} 2-\frac{1}{4} \mathrm{~S} 1} & \text { für E-MMA } \\
F_{\mathrm{A}}=\frac{\mathrm{S} 1}{\frac{1}{2} \mathrm{~S} 2-\mathrm{S} 1} & \text { für E-EMA } \\
F_{\mathrm{A}}=\frac{\mathrm{S} 1}{\frac{1}{2} \mathrm{~S} 2-2 \cdot \mathrm{S} 1} & \text { für E-BMA } \\
F_{\mathrm{A}}=\frac{\mathrm{S} 2}{\frac{1}{2} \mathrm{~S} 3-\frac{1}{4} \mathrm{~S} 2} & \text { für E-BzMA (Methode A) } \\
F_{\mathrm{A}}=\frac{\mathrm{S} 1}{\frac{5}{4} \mathrm{~S} 3-\frac{1}{4} \mathrm{~S} 1} & \text { für E-BzMA (Methode B) } \\
F_{\mathrm{A}}=\frac{\mathrm{S} 1}{\frac{1}{2} \mathrm{~S} 2-\frac{1}{4} \mathrm{~S} 1} & \text { für E-EA } \\
F_{\mathrm{A}}=\frac{\mathrm{S} 1}{\frac{1}{2} \mathrm{~S} 2-\frac{3}{4} \mathrm{~S} 1} & \text { für E-PA }
\end{array}
$$


Die ${ }^{1} \mathrm{H}-\mathrm{NMR}$ Spektren wurden auf einem Varian Unity $300 \mathrm{MHz}$ Spektrometer in 1,1,2,2Tetrachlorethan- $\mathrm{d}_{2}\left(\mathrm{C}_{2} \mathrm{D}_{2} \mathrm{Cl}_{4}, 2\right.$ Gew.- $\%$ Polymer $)$ bei $120^{\circ} \mathrm{C}$ aufgenommen und auf den Solvenspeak bei 5.98 ppm relativ zu TMS referenziert. Die Messungen wurden mit einem Pulswinkel von $45^{\circ}$ und einer Aufnahmezeit pro Puls (at) von $2.66 \mathrm{~s}$ ohne Delay ausgeführt. Die spektrale Breite $(s w)$ lag bei 61.7 kHz. Die typische Messzeit betrug 2 Min.

\subsubsection{Bestimmung der Copolymerzusammensetzung mittels Elementar- analyse}

Die Richtigkeit der ${ }^{1} \mathrm{H}-\mathrm{NMR}$-spektroskopisch bestimmten Copolymerzusammensetzungen wurde stichprobenartig mit einer zweiten absoluten Methode (Elementaranalyse) geprüft.

Die Elementaranalysen der synthetisierten Polymerproben wurden vom Mikroanalytischen Laboratorium Beller (Göttingen) durchgeführt. Der Stoffmengenanteil (Meth)Acrylat im Copolymer $F_{\mathrm{A}}$ berechnet sich nach folgenden Beziehungen entweder aus der Massenbilanz des Sauerstoffs (3.9) oder jener des Kohlenstoffs (3.10):

$$
\begin{gathered}
F_{\mathrm{A}}=\frac{M_{\mathrm{E}} \cdot m_{\mathrm{O}}}{2 \cdot M_{\mathrm{O}}-\left(M_{\mathrm{A}}-M_{\mathrm{E}}\right) \cdot m_{\mathrm{O}}} \\
F_{\mathrm{A}}=\frac{M_{\mathrm{E}} \cdot m_{\mathrm{C}}-2 \cdot M_{\mathrm{c}}}{(\alpha-2) \cdot M_{\mathrm{C}}-\left(M_{\mathrm{A}}-M_{\mathrm{E}}\right) \cdot m_{\mathrm{C}}}
\end{gathered}
$$

$M_{\mathrm{O}}, M_{\mathrm{C}}, M_{\mathrm{E}}, M_{\mathrm{A}}$ sind die molaren Massen von Sauerstoff, Kohlenstoff, Ethen und (Meth)Acrylat. Der Parameter $\alpha$ beschreibt die Anzahl der Kohlenstoffatome im Comonomer (Meth)Acrylat. $m_{\mathrm{O}}$ und $m_{\mathrm{C}}$ sind die elementaranalytisch bestimmten Massenanteile von Sauerstoff und Kohlenstoff in $\frac{1}{100}$ Gew.\%. Die aus den Analyseergebnissen der Elemente Sauerstoff und Kohlenstoff erhaltenen Copolymerzusammensetzungen weichen in allen Fällen um weniger als 2 mol-\% voneinander ab. Im überwiegenden Anteil werden Abweichungen kleiner als 1 mol-\% beobachtet. Zum Vergleich mit den spektroskopisch bestimmten Zusammensetzungen werden die Ergebnisse der Elementaranalyse arithmetisch gemittelt.

Tabelle 3.3 zeigt für verschiedene Copolymersysteme die jeweils elementaranalytisch erhaltenen Zusammensetzungen im Vergleich zu den ${ }^{1}$ H-NMR-spektroskopisch ermittelten Ergebnissen. Die Elementaranalyse ergibt bis auf eine Ausnahme jeweils geringfügig 
größere Zahlenwerte für die Copolymerzusammensetzung. Die Abweichungen betragen für den überwiegenden Teil der Proben weniger als 1 mol-\%, so dass man die Übereinstimmung der Resultate der beiden unabhängigen Methoden als gut bezeichnen kann.

\begin{tabular}{cccc}
\hline \hline \multirow{2}{*}{ Copolymer } & $\begin{array}{c}F_{\mathrm{A}}[\mathrm{mol}-\%] \text { nach } \\
\text { Elementaranalyse }\end{array}$ & $\begin{array}{c}F_{\mathrm{A}}\left[\mathrm{mol} \text {-\%] nach }{ }^{1} \mathrm{H}-\right. \\
\text { NMR-Spektroskopie }\end{array}$ & $\begin{array}{c}\Delta F_{\mathrm{A}} \\
{[\mathrm{mol} \%]}\end{array}$ \\
\hline \multirow{3}{*}{ E-MMA } & 7.5 & 5.8 & 1.7 \\
& 30.4 & 29.8 & 0.6 \\
& 50.1 & 49.6 & 0.5 \\
E-EMA & 53.7 & 52.2 & 1.5 \\
& 2.3 & 2.9 & 0.6 \\
& 31.1 & 30.0 & 1.1 \\
& 47.7 & 47.1 & 0.6 \\
\hline \multirow{3}{*}{ E-EA } & 4.0 & 3.9 & 0.1 \\
& 12.9 & 12.0 & 0.9 \\
& 24.9 & 24.7 & 0.2 \\
& 44.3 & 44.0 & 0.2 \\
\hline
\end{tabular}

Tabelle 3.3: $\quad$ Vergleich der aus Elementaranalyse und ${ }^{1} H$-NMR-Spektroskopie erhaltenen Copolymerzusammensetzungen für E-MMA-, E-EMA- und E-EA-Copolymere

\subsubsection{Bestimmung der Zusammensetzung von Ethen-Methacrylsäure- Butylmethacrylat-Terpolymeren}

Im Rahmen der vorliegenden Arbeit wurden nach zwei verschiedenen Methoden Terpolymere aus Ethen, Methacrylsäure und Butylmethacrylat hergestellt. Zum einen wurden Proben analog der in Abschnitt 3.1 detailliert beschriebenen kontinuierlichen Copolymersynthese nach dem Hochdruck-Verfahren erhalten. Zum anderen wurden Terpolymere durch partielle Veresterung von Ethen-Methacrylsäure-Copolymer mit Butanol synthetisiert. Die Zusammensetzung der Proben wurde in beiden Fällen ${ }^{1}$ H-NMR-spektroskopisch ermittelt.

Eine direkte Analyse der säurehaltigen Terpolymere ist wegen deren schlechter Löslichkeit in organischen Solventien nicht möglich. Durch vollständige Veresterung der Methacrylsäuregruppen mit Diazomethan (Details der Methode s.u.) gelingt es jedoch, ein in einem 
für die NMR-Spektroskopie gebräuchlichen Lösungsmittel (1,1,2,2-Tetrachlorethan- $\left.\mathrm{d}_{2}\right)$ lösliches Terpolymer zu erhalten. Gleichzeitig wird mit der Methylesterfunktion eine mit der NMR-Spektroskopie gut detektierbare Gruppe eingeführt. Der besondere Vorteil liegt hierbei darin, dass das Signal der Methylester-Protonen im NMR-Spektrum deutlich getrennt von jenem der Protonen der Butylestergruppierung auftritt. Dies ermöglicht eine quantitative Bestimmung des Gehalts der einzelnen Comonomere nebeneinander.

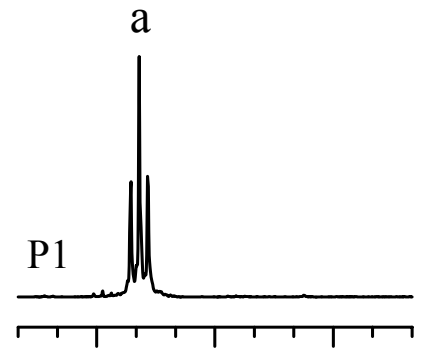

$\begin{array}{lll}4.2 & 3.9 & 3.6\end{array}$

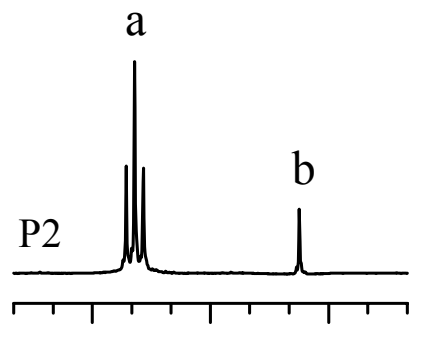

$\begin{array}{lll}4.2 & 3.9 & 3.6\end{array}$

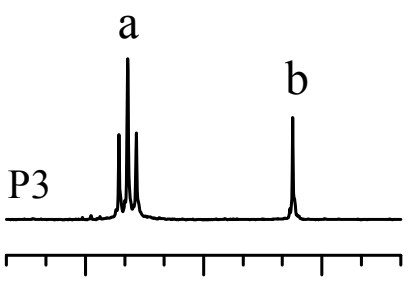

4.2

3.6 $\delta / \mathrm{ppm}$

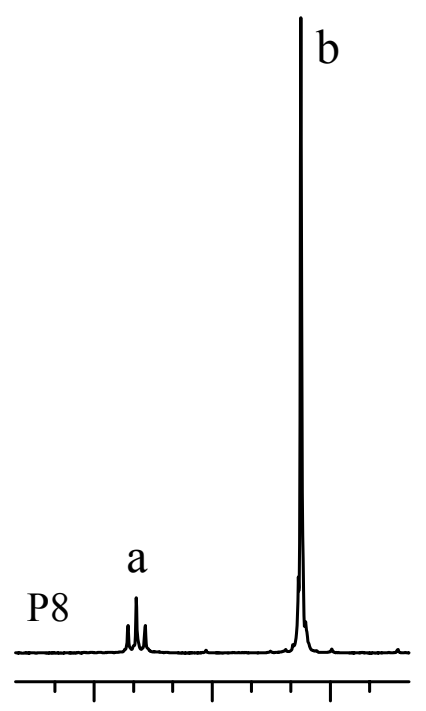

$\begin{array}{lll}4.2 & 3.9 & 3.6\end{array}$

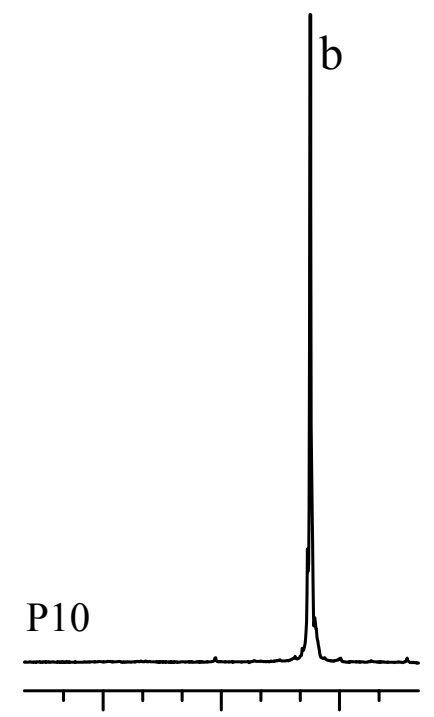

$\begin{array}{lll}4.2 & 3.9 & 3.6\end{array}$

$\delta / \mathrm{ppm}$

Abbildung 3.5: $\quad{ }^{1}$ H-NMR-Spektren einiger Produkte von Ethen-Methacrylsäure-Butylmethacrylat-Terpolymeren nach Veresterung mit Diazomethan. Der Gesamtcomonomergehalt beträgt jeweils ca. 7.5 mol-\%. Der Anteil an Butylmethacrylat in den Proben nimmt von P1 nach P10 zu, der Anteil Methylmethacrylat (aus veresterter Methacrylsäure) nimmt ab. Entsprechend steigt die Intensität des $\mathrm{O}-\mathrm{CH}_{2}$-Signals (b) (3.60 - $3.74 \mathrm{ppm}$ ), gleichzeitig sinkt die Intensität des $\mathrm{O}-\mathrm{CH}_{3}$-Signals (a) (4.00 - 4.20 ppm). 
In Abbildung 3.5 wird die hier angewandte Analysemethode demonstriert. Dargestellt ist eine Serie im Bereich der Absorbanz der $\alpha$-Protonen der Esterseitenkette gedehnter ${ }^{1} \mathrm{H}$ NMR-Spektren von Terpolymeren aus Ethen, Methylmethacrylat und Butylmethacrylat. Die Proben wurden durch Umsetzung von Ethen-Methacrylsäure-Butylmethacrylat-Terpolymeren mit Diazomethan erhalten. Beginnend von links oben nimmt (von Probe P1 bis P10) der Gehalt der Proben an Butylmethacrylat zu und gleichzeitig jener an Methylmethacrylat ab. Dementsprechend steigt die Intensität des O-CH -Signals (b) bei gleichzeitig abnehmender Intensität des O-CH${ }_{3}$-Signals (a). Bei den Auftragungen für Probe P1 und P10 handelt es sich jeweils um ein Spektrum des entsprechenden Randsystems Ethen-MethylmethacrylatCopolymer bzw. Ethen-Butylmethacrylat-Copolymer. Der Gesamtcomonomergehalt beträgt bei allen Proben $7.5 \pm 0.3$ mol-\%. Die zur Berechnung der Terpolymerzusammensetzung verwendeten Integrationsgrenzen sind für die verschiedenen Signale bzw. Signalgruppen in Tabelle 3.4 aufgeführt.

Zur Ermittlung der Zusammensetzung wird zunächst der Gesamt-Ethengehalt $n_{\mathrm{E}}$ der Proben bestimmt. Dieser berechnet sich nach:

$$
n_{\mathrm{E}}(\mathrm{E}-\mathrm{BMA}-\mathrm{MMA})=\frac{1}{4} \mathrm{~S} 3-\frac{3}{2} \mathrm{~S} 1-\frac{5}{3} \mathrm{~S} 2
$$

Für die Comonomergehalte $F_{\mathrm{BMA}}$ und $F_{\mathrm{MAS}}$ ergibt sich somit:

$$
\begin{aligned}
F_{\text {BMA }} & =\frac{\mathrm{S} 1}{\frac{1}{2} \mathrm{~S} 3-\frac{1}{6} \mathrm{~S} 2-2 \cdot \mathrm{S} 1} \\
F_{\mathrm{MAS}} & =\frac{\mathrm{S} 2}{\frac{3}{4} \mathrm{~S} 3-\frac{1}{4} \mathrm{~S} 1-3 \cdot \mathrm{S} 1}
\end{aligned}
$$

Der Gesamtcomonomergehalt berechnet sich als Summe aus $F_{\text {MAS }}$ und $F_{\text {BMA }}$ :

$$
F_{\mathrm{A}}=F_{\mathrm{MAS}}+F_{\mathrm{BMA}}
$$




\begin{tabular}{ccc}
\hline \hline Strukturelement & Bezeichnung & Integrationsgrenzen [ppm] \\
\hline \hline $\mathrm{O}-\mathrm{CH}_{3}$ & $\mathrm{~S} 2$ & $4.00-4.20$ \\
$\mathrm{O}-\mathrm{CH}_{2} \mathrm{CH}_{2} \mathrm{CH}_{2}-\mathrm{CH}_{3}$ & $\mathrm{~S} 1$ & $3.60-3.74$ \\
\hline Hauptkette, & & $0.70-2.40$ \\
$\mathrm{O}-\mathrm{CH}_{2} \mathrm{CH}_{2} \mathrm{CH}_{2}-\mathrm{CH}_{3}$, & $\mathrm{S} 3$ & \\
$\alpha-\mathrm{CH}_{3}$ & & \\
\hline
\end{tabular}

Tabelle 3.4: $\quad$ Signalzuordnung und Integrationsgrenzen für die Bestimmung der Zusammensetzung von Terpolymeren aus Ethen, methylveresterter Methacrylsäure und Butylmethacrylat mittels ${ }^{1} H$-NMR-Spektroskopie.

\section{Veresterung von Ethen-Methacrylsäure-Butylmethacrylat-Copolymeren mit Diazo- methan}

Für die NMR-spektroskopische Untersuchung wurden die Ethen-Methacrylsäure-Butylmethacrylat-Terpolymere mit Diazomethan $\mathrm{zu}$ den entsprechenden Ethen-Methylmethacrylat-Butylmethacrylat-Terpolymeren verestert. Ausgehend von $N$-Methyl- $N$-nitroso-4sulfonamid wurde Diazomethan nach einer von Black [12] beschriebenen Methode hergestellt und hierbei in etherischer Lösung erhalten. Das Polymer wird vor der Umsetzung mit einem Überschuss Tetrahydrofuran versetzt und über Nacht quellen gelassen. Hiernach gibt man bei Zimmertemperatur tropfenweise soviel Diazomethanlösung hinzu, bis die orangegelbe Färbung der Lösung gerade erhalten bleibt. Das Probengefäß wird abgedeckt, jedoch aufgrund der Stickstoffentwicklung nicht fest verschlossen. Nach etwa einstündigem Durchmischen der Probe mittels eines Schwingbodenrührers lässt man überschüssiges Diazomethan und Lösungsmittel abdampfen. Die Probe wird anschließend durch viertägiges Trocknen unter vermindertem Druck $\left(\leq 10^{-3} \mathrm{mbar}\right)$ von Lösungsmittelresten befreit. 


\subsubsection{FT-IR-spektroskopische Untersuchungen}

Als eine wichtige Technik der Polymeranalytik ist die IR-Spektroskopie geeignet, um Informationen über Verzweigungen, Vernetzungen, Kristallinität und Restmonomergehalt zu erhalten [13, 14, 15]. Für Copolymere ist es zudem möglich, die Zusammensetzung IRspektroskopisch zu ermitteln.

In dieser Arbeit wurden von den untersuchten Copolymersystemen IR/NIR-Spektren aufgenommen, um Korrelationen mit den ${ }^{1} \mathrm{H}-\mathrm{NMR}$-spektroskopisch bestimmten Copolymerzusammensetzungen zu ermitteln. Derartige Korrelationen können einerseits als Maß für die Güte der durch die absolute Methode ${ }^{1} \mathrm{H}-\mathrm{NMR}$ erhaltenen Ergebnisse dienen, da eventuell auftretende "Ausreißer“ leichter erkannt werden können. Darüber hinaus können, wenn einmal eine Korrelation aufgestellt ist, aufgrund der für die IR-Spektroskopie im Vergleich zu NMR-Experimenten weniger aufwendigen Probenvorbereitung, weitere Proben erheblich schneller bezüglich ihres Gehalts untersucht werden,

Eine weitere Anwendung ergibt sich bei der Kontrolle der Stabilisierung der für die Trübungsdruckmessungen eingesetzten Copolymere. Bei diesen Messungen werden die aus Ethen und Copolymer bestehenden Mischungen über mehrere Stunden hohen Drücken und Temperaturen ausgesetzt, gerade jenen Bedingungen bei denen üblicherweise auch die Synthese ausgeführt wird. Durch Zugabe von Inhibitor und durch Verwendung von hochreinem Ethen versucht man chemische Reaktionen zu verhindern. Die erfolgreiche Stabilisierung kann durch den Vergleich der Copolymerspektren vor und nach den Messungen geprüft werden (vgl. Abschnitt 5.3).

Zur Ermittlung von Korrelationen wurden in dieser Arbeit zwei Methoden angewandt. In Abschnitt 3.2.4.1 wird zunächst eine Vorgehensweise beschrieben, welche analog einer Methode von Buback et al. [16] das Verhältnis charakteristischer Absorbanzen der Comonomergruppen im IR-Spektrum verwendet. Nachfolgend wird die Anwendung einer chemometrischen Methode der sogenannten „multivariaten Kalibration“ erläutert (Abschnitt 3.2.4.2). Abschließend wird die Vorhersagegenauigkeit der beiden Methoden verglichen.

Die für beide Methoden verwendeten IR-Spektren wurden an zu Filmen verarbeiteten Polymerproben aufgenommen. Diese Polymerfolien wurden mit einer beheizten, hydraulischen Presse (Specac Ltd.) hergestellt. Hierzu werden 20 - 100 mg des Polymers zwischen zwei Teflonfolien von $0.1 \mathrm{~mm}$ Stärke in ein Presswerkzeug gelegt und zwischen die Druckplatten der Presse gebracht. Das Polymer wird darin auf $150{ }^{\circ} \mathrm{C}$ erhitzt und mit einer Kraft von $3 \cdot 10^{6} \mathrm{~N}$ gepresst. Nach etwa 2 min. wird das Presswerkzeug entnommen 
und in einem wassergekühltem Einsatz auf Zimmertemperatur temperiert. Die erhaltene Polymerfolie wird vorsichtig von den Teflonfolien getrennt und auf einem Kartonträger fixiert. Im Fall von Proben mit hohem Gehalt an (Meth)Acrylat (mit Ausnahme von Methylmethacrylat) tritt z.T. das Problem auf, dass sich die Teflonfolien aufgrund des zähflüssig klebrigen Charakters dieser Copolymere nicht abziehen lassen, ohne die gepressten Filme gleich wieder zu zerstören. Zur Umgehung dieser Problematik haben sich folgende Vorgehensweisen bewährt:

a) In einigen Fällen gelingt es, die Proben mitsamt den beiden Teflonfolien durch Eintauchen in flüssigen Stickstoff kurzzeitig so weit zu verhärten, dass sie vom Teflonmaterial getrennt werden können.

b) Spektren von sehr weichen Proben können an Probenmaterial aufgenommen werden, welches durch Zusammenpressen zwischen zwei $\mathrm{CaF}_{2}$-Fenstern in Filmform vorliegt.

c) Die Proben lassen sich mit den anhaftenden Teflonfolien vermessen. Hierbei muss das Ergebnisspektrum noch um die Absorbanz der Teflonfolien korrigiert werden. Da die Teflonfolien z.T. durch das Pressen ebenfalls dünner werden, kann es erforderlich sein, eine Gewichtung der Teflonspektren vorzunehmen.

Die vorbereiteten Polymerfilme werden auf einem Halter in den Probenraum des Spektrometers gebracht. Die IR-Spektren werden mit einem Fourier-Transform-Spektrometer (Bruker IFS 88) aufgenommen, dessen Innenraum kontinuierlich mit wasser- und kohlendioxidarmer Pressluft gespült wird. Es wird folgender optischer Aufbau verwendet: Globar-Lichtquelle, Silicium-beschichteter Calciumfluorid-Strahlteiler und DTGS-Detektor. Die Spektrenaufnahme und -verarbeitung wird über einen PC mit dem Programm OPUS ${ }^{\circledR}$ (Spektrometer-Software, Bruker) gesteuert. Die im Wellenzahlenbereich von 1300 bis $8000 \mathrm{~cm}^{-1}$ ermittelten Spektren werden durch Coaddition von 32 Interferogrammen, mit anschließender Fourier-Transformation (Blackman-Harris 3-Term Apodisierungsfunktion) zur Bestimmung des Einkanalspektrums und Berechnung des Absorbanzspektrums mit dem Leerkanalspektrum erhalten. 


\subsubsection{Univariate NIR/IR-NMR-Korrelationen}

Die optische Schichtlänge der für die Spektrenmessungen hergestellten Polymerfilme liegt zwischen ca. 0.1 und $0.6 \mathrm{~mm}$. Sie kann allerdings nicht mit ausreichender Genauigkeit bestimmt werden. Aus diesem Grund lässt sich die Copolymerzusammensetzung nicht direkt aus den für die einzelnen Comonomereinheiten charakteristischen Banden bestimmen. Buback et al. haben am Beispiel der Copolymersysteme Ethen-Butylacrylat und Ethen-2-Ethylhexylacrylat eine Methode vorgestellt, die das Verhältnis zweier Absorbanzen mit den unabhängig bestimmten Copolymerzusammensetzungen korreliert [16]. Im folgenden werden diese Methode und die zur Anwendung auf die in dieser Arbeit untersuchten Copolymersysteme notwendigen Modifikationen beschrieben.

In Abbildung 3.6 ist (mit Ausnahme von E-MMA) für jedes der untersuchten Copolymersysteme E-EA, E-PA, E-EMA und E-BzMA beispielhaft ein IR/NIR-Absorptionsspektrum im Bereich von $4600-2200 \mathrm{~cm}^{-1}$ dargestellt. Der (Meth)Acrylatgehalt der jeweiligen Probe ist in der zugehörigen Bildunterschrift angegeben. Die in allen vier Spektren auftretende wenig strukturierte Absorption im Bereich von etwa $2770-2440 \mathrm{~cm}^{-1}$ wird durch $\mathrm{CH}-$ Schwingungen hervorgerufen. Das schraffiert dargestellte Integral dieser Bande ist proportional zum C-H-Anteil im jeweiligen Copolymer. Das in jedem Spektrum erkennbare scharfe Signal mit einem Maximum bei ca. $3440 \mathrm{~cm}^{-1}$ ist der Absorption des ersten Obertons der Carbonylstreckschwingung zuzuordnen. Das Integral dieser Bande (ebenfalls schraffiert dargestellt) ist proportional zum (Meth)Acrylatgehalt des jeweiligen Copolymers.

Die zur Integration der Signale verwendeten Grenzen sind in Tabelle 3.5 angegeben. Die Grundlinie des $\mathrm{C}=\mathrm{O}$-Absorbanz-Integrals wurde in allen Fällen durch eine Gerade, welche die Absorbanz bei der unteren und oberen Integrationsgrenze verbindet, festgelegt. Der Integrationsbereich der $\mathrm{CH}$-Bande wird durch eine horizontale Grundlinie, welche bei der Absorbanz an der unteren Integrationsgrenze beginnt, begrenzt. Die geringfügigen Unterschiede der gewählten Integrationsgrenzen berücksichtigen die leicht variierende Lage der Absorbanzen in den betrachteten Copolymerspektren. 

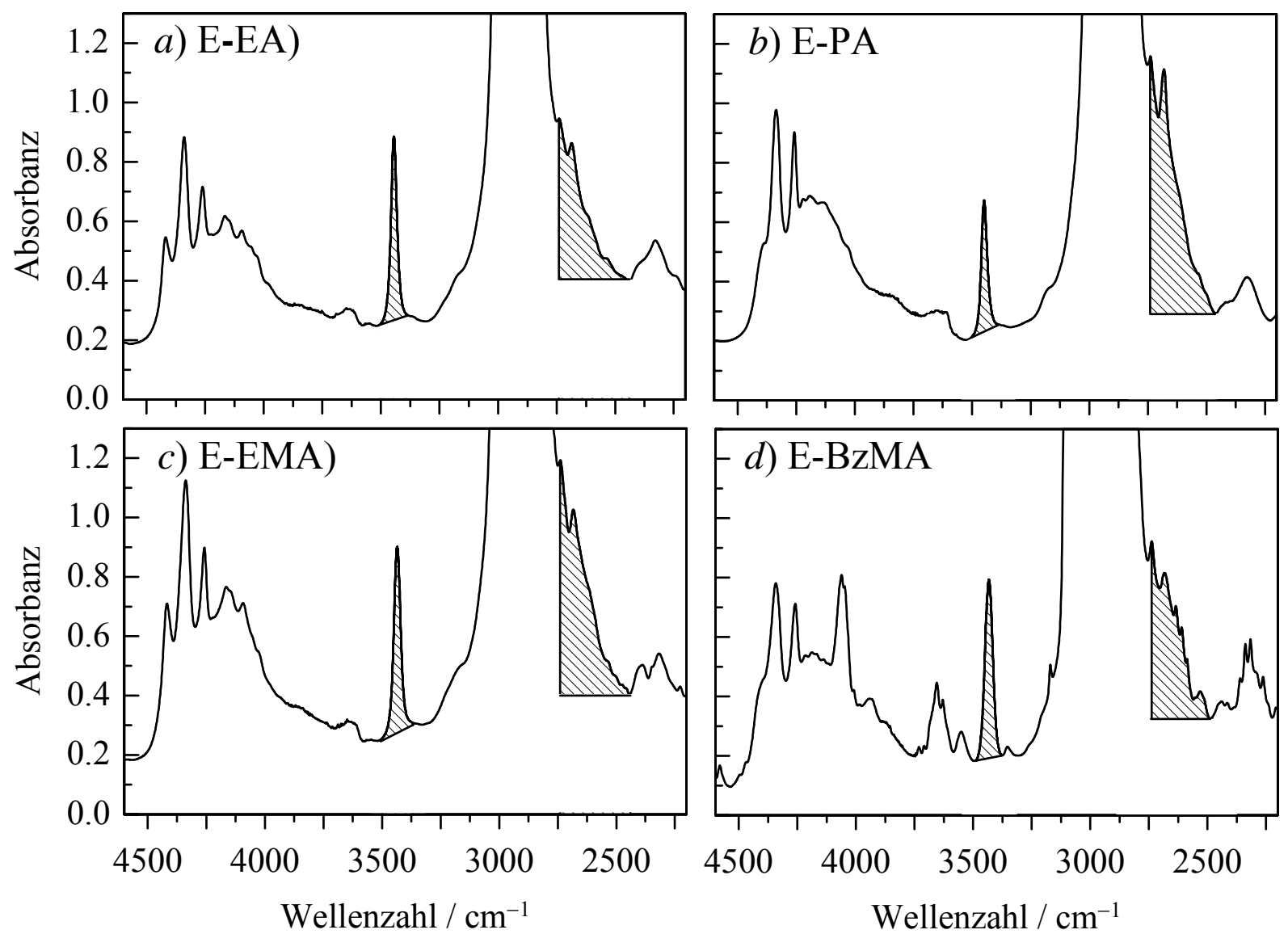

Abbildung 3.6: IR/NIR-Absorptionsspektren von: a) E-EA mit einem Acrylatgehalt von 28.9 mol-\%, b) E-PA mit einem Acrylatgehalt von 14.1 mol-\%, c) E-EMA mit einem Methacrylatgehalt von $24.2 \mathrm{~mol}-\%$, und E-BzMA mit einem Methacrylatgehalt von $26.1 \mathrm{~mol}-\%$. Die für die Auswertung verwendeten Integrationsbereiche sind schraffiert gezeichnet.

\begin{tabular}{ccc}
\hline \hline Copolymersystem & $\begin{array}{c}\text { Integrationsgrenzen der } \\
\mathrm{C}=\mathrm{O}-\text { Absorption } / \mathrm{cm}^{-1}\end{array}$ & $\begin{array}{c}\text { Integrationsgrenzen der } \\
\mathrm{C}-\mathrm{H} \text {-Absorption } / \mathrm{cm}^{-1}\end{array}$ \\
\hline \hline E-EA & $3500-3390$ & $2740-2440$ \\
E-PA & $3500-3390$ & $2740-2460$ \\
E-EMA & $3495-3380$ & $2770-2440$ \\
E-BzMA & $3485-3380$ & $2738-2480$ \\
\hline
\end{tabular}

Tabelle 3.5: Integrationsgrenzen der zur Analyse der Copolymerzusammensetzung verwendeten charakteristischen $\mathrm{C}-\mathrm{H}$ - und $\mathrm{C}=\mathrm{O}$-Bande für verschiedene Ethen-(Meth)Acrylat-Copolymersysteme. 
Entsprechend der Vorgehensweise von Buback et al. wird das Verhältnis der in Abbildung 3.6 hervorgehoben dargestellten charakteristischen Absorbanzen mit der ${ }^{1} \mathrm{H}-\mathrm{NMR}$-spektroskopisch bestimmten Copolymerzusammensetzung korreliert.

Für den Ausgleich der korrelierten Datenpaare eignet sich Gleichung 3.15. $\operatorname{Int}(\mathrm{C}=\mathrm{O})$ bezeichnet hierbei das Integral des Carbonyl-Signals und $\operatorname{Int}(\mathrm{C}-\mathrm{H})$ steht für das Integral der $\mathrm{CH}-\mathrm{Absorbanz}$. Die Parameter $a$ und $b$ werden durch nichtlineare Regression aus den in Abbildung 3.7 dargestellten Auftragungen des Intensitätsverhältnisses $\operatorname{Int}(\mathrm{C}=\mathrm{O}) / \operatorname{Int}(\mathrm{C}-\mathrm{H})$ gegen die Copolymerzusammensetzung $F_{\mathrm{A}}$ bestimmt.

$$
\frac{\operatorname{Int}(\mathrm{C}=\mathrm{O})}{\operatorname{Int}(\mathrm{C}-\mathrm{H})}=\frac{1}{a+F_{\mathrm{A}} \cdot b} \cdot F_{\mathrm{A}}
$$

Gleichung 3.15 leitet sich aus spektroskopischen Größen ab. So kann der Quotient aus Sauerstoff- und Wasserstoffkonzentration im Copolymer nach Gleichung 3.16 anhand der zuvor erläuterten IR/NIR-Absorbanzen und den zugehörigen Molarabsorptivitäten (G1. 3.16 mittlerer Term) berechnet werden. Der Quotient lässt sich außerdem aus der NMR-spektroskopisch bestimmten Copolymerzusammensetzung $F_{\mathrm{A}}$ berechnen (G1. 3.16 rechter Term). Der Faktor $\beta$ bezeichnet die Anzahl an Wasserstoffatomen im jeweiligen (Meth)Acrylatmonomer.

$$
\frac{c_{\mathrm{O}}}{c_{\mathrm{H}}}=\frac{2 \cdot \operatorname{Int}(\mathrm{C}=\mathrm{O}) \cdot B_{\mathrm{C}=\mathrm{O}}^{-1}}{\operatorname{Int}(\mathrm{CH}) \cdot B_{\mathrm{CH}}{ }^{-1}}=\frac{2 \cdot F_{\mathrm{A}}}{4+(\beta-4) \cdot F_{\mathrm{A}}}
$$

Durch den Faktor 2 wird berücksichtigt, dass spektroskopisch nur die Sauerstoffatome der Carbonylgruppe erfasst werden, jedoch in jeder Carbonylgruppe der (Meth)Acrylateinheiten ein weiteres Sauerstoffatom in der Esterfunktionalität vorhanden ist. $B_{\mathrm{C}=\mathrm{O}}$ ist die integrale Molarabsorptivität des ersten Obertons der Carbonylstreckschwingung. $\bar{B}_{\mathrm{CH}}$ bezeichnet die der $\mathrm{CH}$-Schwingung bei etwa $2740-2440 \mathrm{~cm}^{-1}$ zugeordnete integrale Molarabsorptivität. Die Absorption in diesem Bereich wird durch $\mathrm{CH}$-Bindungen der Ethylen- und der (Meth)Acrylateinheiten im Copolymer hervorgerufen. Es ist $\mathrm{zu}$ erwarten, dass sich die integrale Molarabsorptivität der $\mathrm{CH}-$ Bande im Polyethylen $\left(B_{\mathrm{CH}, \mathrm{PE}}\right)$ von der des Poly(meth)acrylats $\left(B_{\mathrm{CH}, \mathrm{PA}}\right)$ unterscheidet. Für die Berechnung der integralen Molarabsorptivität 
von $\mathrm{CH}$ im Copolymer $\left(\bar{B}_{\mathrm{CH}}\right)$ wird daher die Polymerkette entsprechend der Copolymerzusammensetzung $F_{\mathrm{A}}$ in Polyethylen-ähnliche und Poly(meth)acrylat-ähnliche Segmente unterteilt (Gleichung 3.17). $B_{\mathrm{CH}, \mathrm{PE}}$ wird dabei der Absorption der $\mathrm{CH}$-Bindungen in den Ethyleneinheiten zugeordnet und $B_{\mathrm{CH}, \mathrm{PA}}$ der Absorption aller $\mathrm{CH}$-Bindungen in den (Meth)Acrylateinheiten.

$$
\bar{B}_{\mathrm{CH}}=\frac{\left(1-F_{\mathrm{A}}\right) \cdot 4 \cdot B_{\mathrm{CH}, \mathrm{PE}}+F_{\mathrm{A}} \cdot \beta \cdot B_{\mathrm{CH}, \mathrm{PA}}}{\left(1-F_{\mathrm{A}}\right) \cdot 4+F_{\mathrm{A}} \cdot \beta}
$$

Aus der Kombination von Gleichung 3.16 und 3.18 ergibt sich folgender Zusammenhang zwischen dem Verhältnis der Integrale der IR-Banden $\operatorname{Int}(\mathrm{C}=\mathrm{O}) / \operatorname{Int}(\mathrm{CH})$ und der NMRspektroskopisch bestimmten Copolymerzusammensetzung $F_{\mathrm{A}}$ :

$$
\frac{\operatorname{Int}(\mathrm{C}=\mathrm{O})}{\operatorname{Int}(\mathrm{C}-\mathrm{H})}=\frac{B_{\mathrm{C}=\mathrm{O}}}{4 \cdot B_{\mathrm{CH}, \mathrm{PE}}+F_{\mathrm{A}} \cdot\left(\beta \cdot B_{\mathrm{CH}, \mathrm{PA}}-4 \cdot B_{\mathrm{CH}, \mathrm{PE}}\right)} \cdot F_{\mathrm{A}}
$$

Gleichung 3.18 lässt sich durch Einführen von Parameter $a$ und $b$ vereinfachen:

$$
a=\frac{4 \cdot B_{\mathrm{CH}, \mathrm{PE}}}{B_{\mathrm{C}=\mathrm{O}}} \quad b=\frac{\beta \cdot B_{\mathrm{CH}, \mathrm{PA}}-4 \cdot B_{\mathrm{CH}, \mathrm{PE}}}{B_{\mathrm{C}=\mathrm{O}}}
$$

Mit diesen Parametern ergibt sich aus 3.18 Gleichung 3.15 als Endausdruck für die Abhängigkeit des IR-spektroskopisch bestimmten Intensitätsverhältnisses von der Copolymerzusammensetzung.

In Abbildung 3.7 ist für die vier Copolymersysteme Ethen-Ethylacrylat, Ethen- Propylacrylat, Ethen-Ethylmethacrylat und Ethen-Benzylmethacrylat jeweils das Intensitätsver- 
hältnis $\operatorname{Int}(\mathrm{C}=\mathrm{O}) / \operatorname{Int}(\mathrm{C}-\mathrm{H})$ in Abhängigkeit von $F_{\mathrm{A}}$ dargestellt. Die aus der Anpassung von Gleichung 3.15 an die Daten erhaltenen Werte für die Parameter $a$ und $b$ sind in Tabelle 3.6 aufgelistet. Es sei angemerkt, dass auch für reines Polyethylen eine wenn auch nur sehr geringe Absorbanz im Bereich von $3413-3477 \mathrm{~cm}^{-1}$ gefunden wird. Diese Absorbanz ist Carbonylgruppen zuzuordnen, welche durch in geringem Maße eintretende Oxidation des Polymers während oder nach der Synthese entstehen [17].
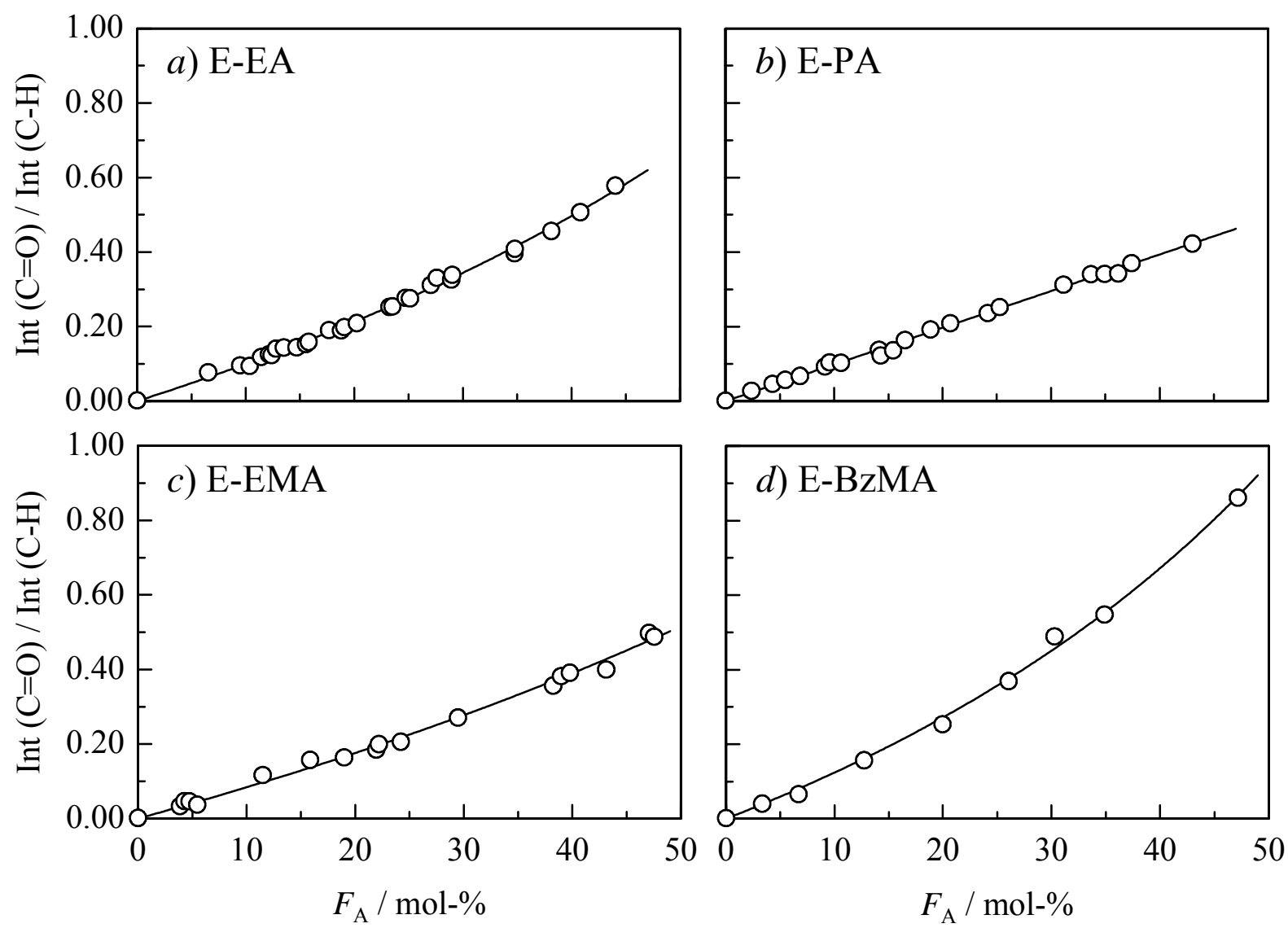

Abbildung 3.7: Verhältnis der integrierten Absorbanzen von charakteristischen Carbonylund $\mathrm{CH}$-Banden, Int $(\mathrm{C}=\mathrm{O}) / \operatorname{Int}(\mathrm{C}-\mathrm{H})$, in Abhängigkeit von der ${ }^{1} \mathrm{H}-\mathrm{NMR}$ spektroskopisch bestimmten Copolymerzusammensetzung $F_{\mathrm{A}}$. Abbildung: a) E-EA-Copolymer, b) E-PA-Copolymer, c) E-EMA-Copolymer und d) EBzMA-Copolymer.

Aus Abbildung 3.7 ist ersichtlich, dass die Verknüpfung der IR-spektroskopischen Ergebnisse mit den Copolymerzusammensetzungen für jedes der vier betrachteten Systeme sinnvoll ist, da jeweils ein eindeutiger Zusammenhang zwischen den Resultaten der voneinander unabhängigen Messmethoden zu erkennen ist. Die aus den Ergebnissen $\operatorname{der}{ }^{1} \mathrm{H}$ NMR-Spektroskopie berechneten Copolymerzusammensetzungen erscheinen somit verlässlich. 
Aus den NIR/IR-Spektren der Copolymere können nicht direkt Werte für die integrale Molarabsorptivität der $\mathrm{C}=\mathrm{O}$-Bande, $B_{\mathrm{C}=\mathrm{O}}$ bestimmt werden, da die Schichtlänge der Polymerfilme nicht exakt bekannt ist. Hingegen lassen sich auf einfache Weise $B_{\mathrm{C}=\mathrm{O}}$-Werte für monomere (Meth)Acrylate, von welchen IR-Spektren in Küvetten aufgenommen werden können, erhalten. Unter der Annahme, dass die an monomeren (Meth)Acrylaten bestimmten Werte für $B_{\mathrm{C}=\mathrm{O}}$ auch für die (Meth)Acrylateinheiten in den Copolymeren gültig sind, können aus den Parametern $a$ und $b$ Werte für die integralen Molarabsorptivitäten $B_{\mathrm{CH}, \mathrm{PE}}$ und $B_{\mathrm{CH}, \mathrm{PA}}$ berechnet werden (vgl. Gleichung 3.19). Die für die untersuchten Copolymersysteme erhaltenen Ergebnisse sind in Tabelle 3.6 aufgeführt. Außerdem enthält Tabelle 3.6 die von Dröge $[10,16]$ bestimmten Werte für die verwandten Copolymersysteme EthenMethylacrylat, Ethen-Butylacrylat, Ethen-2-Ethylhexylacrylat und Ethen-Butylmethacrylat.

\begin{tabular}{ccccccc}
\hline \hline Copolymer & $a$ & $b$ & $\begin{array}{c}B_{\mathrm{C}=\mathrm{O}} \\
{\left[\mathrm{m} \cdot \mathrm{mol}^{-1}\right]}\end{array}$ & $\begin{array}{c}B_{\mathrm{CH}, \mathrm{PE}} \\
{\left[\mathrm{m} \cdot \mathrm{mol}^{-1}\right]}\end{array}$ & $\begin{array}{c}B_{\mathrm{CH}, \mathrm{PA} .} \\
{\left[\mathrm{m} \cdot \mathrm{mol}^{-1}\right]}\end{array}$ & Literatur \\
\hline \hline E-MA & 1.1492 & -0.5302 & $1052 \pm 130$ & $302 \pm 40$ & $109 \pm 32$ & {$[16]$} \\
E-EA & 1.0641 & -0.6432 & $981 \pm 120$ & $261 \pm 35$ & $52 \pm 10$ & d. Arbeit \\
E-PA & 1.0149 & 0.0042 & $1034 \pm 120$ & $262 \pm 35$ & $105 \pm 15$ & d. Arbeit \\
E-BA & 0.9981 & 0.4910 & $1250 \pm 150$ & $311 \pm 45$ & $155 \pm 34$ & {$[16]$} \\
E-EHA & 1.0126 & 2.3558 & $1210 \pm 140$ & $306 \pm 35$ & $204 \pm 30$ & [16] \\
E-EMA & 1.2969 & -0.6408 & $874 \pm 105$ & $283 \pm 35$ & $57 \pm 10$ & d. Arbeit \\
E-BMA & 1.0106 & 0.4499 & $1227 \pm 140$ & $310 \pm 40$ & $128 \pm 34$ & [10] \\
E-BzMA & 0.8766 & 0.7038 & $950 \pm 120$ & $208 \pm 25$ & $13 \pm 5$ & d. Arbeit \\
\hline
\end{tabular}

Tabelle 3.6: Angepasste Parameter $a$ und b, für monomere (Meth)Acrylate bestimmte Werte für $B_{C=O}$ sowie mit Gleichung 3.21 berechnete Werte für $B_{\mathrm{CH}, \mathrm{PE}}$ und $B_{\mathrm{CH}, \mathrm{PA}}$.

Anhand der in Tabelle 3.6 aufgelisteten Ergebnisse lassen sich folgende Feststellungen treffen: i) Die Werte der Parameter $a$ und $b$ folgen mit der Länge der Alkylgruppe der (Meth)Acrylateinheit bei den Ethen-Acrylat- und Ethen-Methacrylatsystemen in gleichartiger Weise einem Trend: Der Zahlenwert von Parameter $a$ wird mit größer werdendem 
Esterrest geringer, während der Zahlenwert von Parameter $b$ in der gleichen Richtung größer wird. Einzig der Wert für $b$ für E-EA-Copolymere ist höher als aufgrund des Trends zu erwarten. Diese Abweichung ist jedoch nur gering und liegt innerhalb der experimentellen Unsicherheit der Daten. ii) Die Werte für die integrale Molarabsorptivität von $\mathrm{CH}$ Schwingungen aus Polyethylen-ähnlichen Segmenten der Copolymere $B_{\mathrm{CH}, \mathrm{PE}}$ liegen sehr dicht an dem für ein LDPE Homopolymer mit bekannter optischer Schichtlänge gefundenen Wert von $B_{\mathrm{CH}, \mathrm{PE}}=325 \pm 33 \mathrm{~m} \cdot \mathrm{mol}^{-1}$ [16]. Sie können unter Berücksichtigung ihrer Unsicherheit praktisch nicht unterschieden werden. Es ist anzunehmen, dass $B_{\mathrm{CH}, \mathrm{PE}}$ unabhängig von dem betrachteten (Meth)Acrylat ist. iii) Die in dieser Arbeit bestimmten Werte für $B_{\mathrm{CH}, \mathrm{PA}}$ ordnen sich nicht in die in der Literatur beobachtete Reihung hinsichtlich der Länge des Esterrestes ein. Zur Angabe eines Trends ist die Genauigkeit der Daten nicht ausreichend. Aufgrund der bei der Auswertung der IR-Spektren für jedes Copolymersystem abgewandelten Integrationsgrenzen und der vergleichsweise großen Unsicherheit der bestimmten Werte erscheint eine weitergehende Interpretation nicht sinnvoll.

Zur Auswertung der IR-Spektren der E-MMA-Copolymere musste im Unterschied zu den zuvor beschriebenen Systemen eine andere Methodik gewählt werden. Die Ursache hierfür wird in Abbildung 3.8 anhand einer Auswahl von E-MMA-Spektren illustriert. Erwartungsgemäß wächst mit zunehmendem Methylmethacrylatgehalt der Copolymere die Intensität des 1. Obertones der Carbonylschwingung bei ca. $3440 \mathrm{~cm}^{-1}$ an und entsprechend nimmt gleichzeitig die Intensität im CH-Schwingungsbereich ab. Vergleicht man die IR-Spektren von E-MMA mit denen der anderen Ethen-Copolymere (Abb. 3.6), fällt deutlich auf, dass in den E-MMA-Spektren zwei Banden bei $3540 \mathrm{~cm}^{-1}$ und $3630 \mathrm{~cm}^{-1}$ auftreten, deren Intensitäten mit dem Methacrylatgehalt stark zunehmen. 


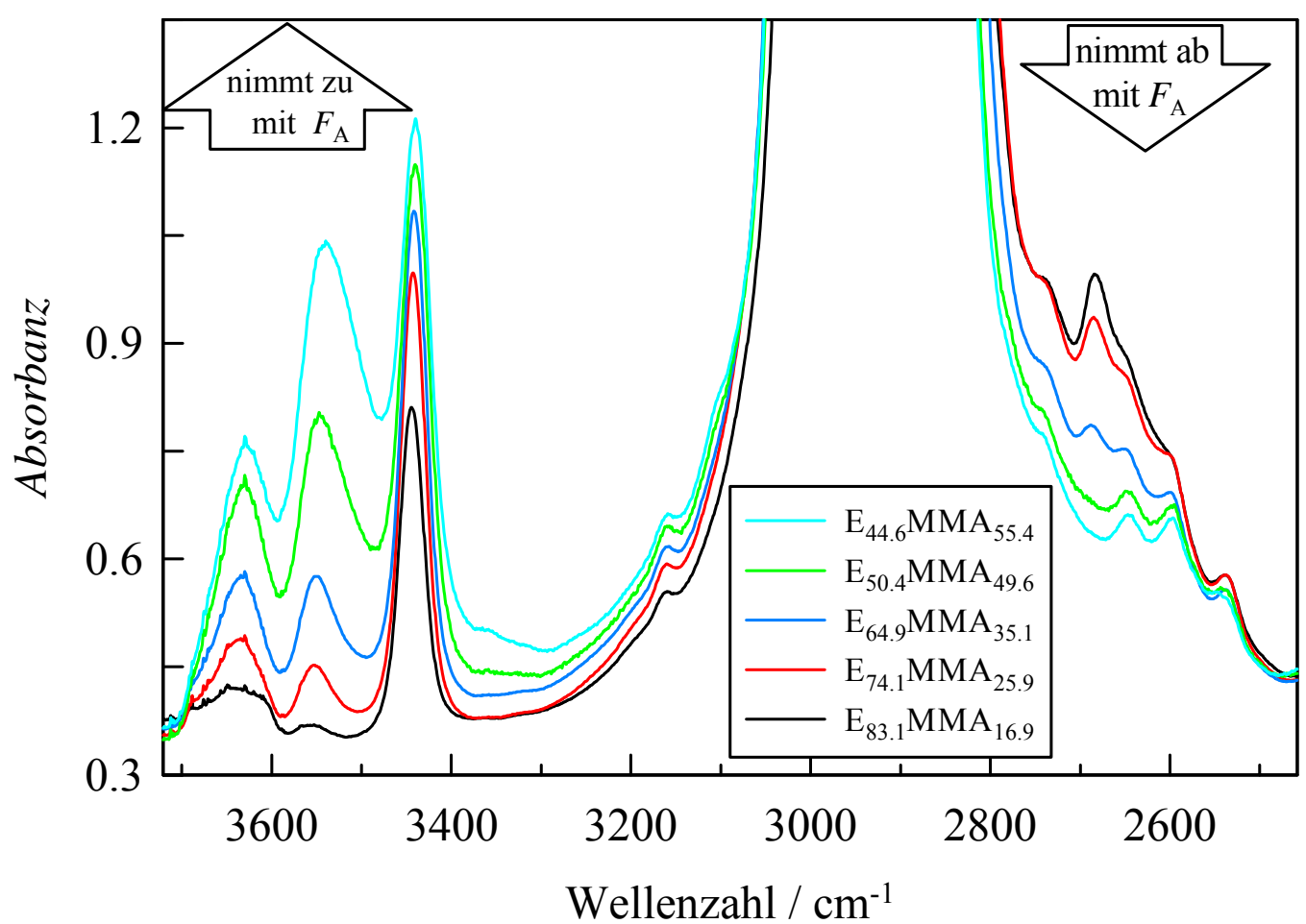

Abbildung 3.8: Eine Serie von IR/NIR-Absorptionsspektren von E-MMA-Copolymeren. Der jeweilige Gehalt der Proben an MMA ist in der Legende aufgeführt.

Diese Banden sind zwar auch in den Spektren von E-BzMA erkennbar und möglicherweise auch untergründig in den Spektren der anderen Systeme vorhanden, stören aber aufgrund ihrer dort nur sehr geringen Intensität die Auswertung bei diesen Systemen nicht. Im Falle des E-MMA-Systems sind diese Banden allerdings ab einem Gehalt von ca. 20 mol-\% nicht mehr von der Carbonylbande grundlinienisoliert und verhindern somit die getrennte Integration.

Um die geschilderte Problematik der Bandenüberlagerung $\mathrm{zu}$ umgehen, bietet sich das Verfahren der Bandenzerlegung an. Für die weitere Auswertung wurde für alle E-MMASpektren im Wellenzahlenbereich von $3300-3850 \mathrm{~cm}^{-1}$ eine computergestützte Bandenanalyse mit dem Softwarepaket OPUS ${ }^{\circledR} 4.0$ (Bruker, Karlsruhe) durchgeführt. Hierzu wurden bei $3365 \mathrm{~cm}^{-1}, 3450 \mathrm{~cm}^{-1}, 3540 \mathrm{~cm}^{-1}, 3630 \mathrm{~cm}^{-1}$ und $3720 \mathrm{~cm}^{-1}$ Stützstellen definiert sowie eine Stützstelle zur Grundlinienbestimmung eingerichtet, wobei jeweils ein Gaußprofil angenommen wurde.

In Abbildung 3.9 a) werden die erhaltenen Resultate am Beispiel des IR/NIR-Absorptionsspektrums von $\mathrm{E}_{44.6} \mathrm{MMA}_{55.4}$ dargestellt. Das mittels Bandenanalyse isolierte Signal des 1. Obertones der Carbonylschwingung ist zur besseren Übersicht mit einem negativen Offset aufgetragen. Zur Integration der Signale wurden die Grundlinien analog der im voran- 
gegangenen Abschnitt erläuterten Vorgehensweise festgelegt. In Abbildung 3.9 b) ist die für E-MMA-Copolymere ermittelte Korrelation dargestellt. Aufgetragen ist das Verhältnis des Integrals des aus der Bandenanalyse erhaltenen CO-Signals zum Integral der CH-Absorbanz in Abhängigkeit von der ${ }^{1} \mathrm{H}-\mathrm{NMR}$ spektroskopisch ermittelten Copolymerzusammensetzung. Nachfolgend sind in Tabelle 3.7 die für das Copolymersystem Ethen-MMA verwendeten Integrationsgrenzen sowie die durch Anpassung nach Gleichung 3.15 für die Korrelation ermittelten Parameter $a$ und $b$ aufgelistet.
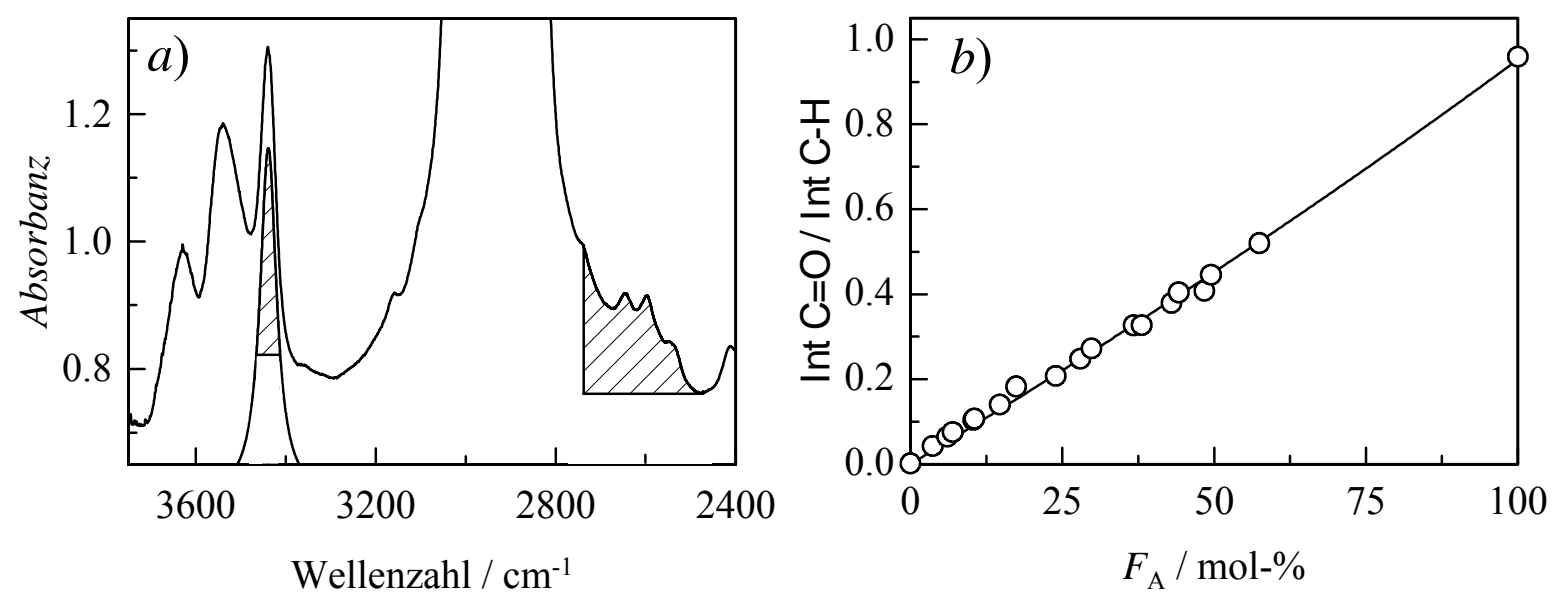

Abbildung 3.9:

a) IR/NIR-Absorptionsspektren von $E_{45} M M A_{55}$. Die für die spektroskopische Bestimmung der Polymerzusammensetzung genutzten Flächen sind schraffiert. Die Integrationsgrenzen sind in Tabelle 3.7 angegeben.

b) Korrelation des Quotienten des $C=O$ - und $C$-H-Bandenintegrals mit den ${ }^{1} H$-NMRspektroskopisch bestimmten Zusammensetzungen für das System E-MMA-Copolymer.

\begin{tabular}{cccc}
\hline \hline$a$ & $b$ & $\begin{array}{c}\text { Integrationsgrenzen } \\
\mathrm{C}=\mathrm{O}-\text { Absorption } / \mathrm{cm}^{-1}\end{array}$ & $\begin{array}{c}\text { Integrationsgrenzen } \\
\mathrm{CH}-\text { Absorption } / \mathrm{cm}^{-1}\end{array}$ \\
\hline \hline 1.1615 & -0.1115 & $3413-3477$ & $2472-2738$ \\
\hline
\end{tabular}

Tabelle 3.7: $\quad$ Parameter $a$ und b für die Korrelation nach Gleichung 3.15 für E-MMACopolymere. Integrationsgrenzen der zur Analyse der Polymerzusammensetzung verwendeten charakteristischen $\mathrm{C}-\mathrm{H}$ - und $\mathrm{C}=\mathrm{O}$-Bande von $\mathrm{E}-\mathrm{MMA}$. 
Auch für dieses System lässt sich ein eindeutiger Zusammenhang zwischen den Ergebnissen der beiden unabhängigen Analysemethoden feststellen. Insgesamt belegen die für die verschiedenen Copolymersysteme erhaltenen Korrelationen und die gefundene gute Übereinstimmung der NMR-spektroskopischen und elementaranalytischen Ergebnisse die Eignung der ${ }^{1}$ H-NMR-Spektroskopie zur Analyse der Copolymerzusammensetzung.

Abschließend sei angemerkt, dass das Verfahren der Bandenanalyse prinzipiell auch für die Aufstellung von Korrelationen bei den anderen untersuchten Copolymersystemen angewendet werden kann. Da jedoch bei den betrachteten Copolymersystemen die Auswertung nach [16] nur im Fall der E-MMA-Copolymere durch überlappende Absorbanzen gestört wird ist dies nicht erforderlich.

\subsubsection{Multivariate NIR/IR-NMR-Korrelationen}

In diesem Abschnitt wird die Anwendung des Verfahrens der sogenannten „multivariaten Kalibrierung“ beschrieben, mit welchem sich ebenfalls NIR/IR-Copolymerspektren mit (unabhängig ermittelten) Copolymerzusammensetzungen korrelieren lassen. Grundprinzip der Methode ist es, bei der Spektrenauswertung nicht nur wie im Fall der univariaten Kalibrierung einzelne Banden bzw. Punkte der Spektren, sondern die spektralen Eigenschaften einer Probe über einen möglichst großen Bereich zu berücksichtigen.

Allgemein hat jedes quantitativ arbeitende analytische Messverfahren die Aufgabe, aus einer messbaren Systemgröße $X$ eine oder mehrere Systemeigenschaften $Y$ quantitativ zu bestimmen. Während der Kalibration wird anhand bekannter Proben ein Zusammenhang zwischen der Messgröße $X$ (hier Absorbanzdaten) und den Systemeigenschaften $Y$ (hier Copolymerzusammensetzung, $F_{\mathrm{A}}$ ) gesucht. Im Falle der multivariaten Kalibrierung wird dieser Zusammenhang durch die folgende Gleichung ausgedrückt, welche die sogenannte Kalibrierfunktion $K$ beinhaltet:

$$
\begin{gathered}
\vec{Y}=X \cdot \vec{K} \\
{\left[\begin{array}{cccc}
y_{11} & y_{12} & \ldots & y_{1 p} \\
y_{21} & y_{22} & \ldots & y_{2 p} \\
. & . & \ldots & \cdot \\
y_{n 1} & y_{n 2} & \ldots & y_{n p}
\end{array}\right]=\left[\begin{array}{cccc}
x_{11} & x_{12} & \ldots & x_{1 p} \\
x_{21} & x_{22} & \ldots & x_{2 p} \\
\cdot & . & \ldots & \cdot \\
x_{n 1} & x_{n 2} & \ldots & x_{n p}
\end{array}\right] \cdot\left[\begin{array}{cccc}
k_{11} & k_{12} & \ldots & k_{1 m} \\
k_{21} & k_{22} & \ldots & k_{2 m} \\
. & . & \ldots & \cdot \\
k_{p 1} & k_{p 2} & \ldots & k_{p m}
\end{array}\right]}
\end{gathered}
$$


Die Matrix $Y$ besteht aus den Eigenschaftswerten der jeweils einzelnen Kalibrierprobe. Die Matrix $Y$ weist bei der hier angewandten Kalibrationsaufgabe nur eine Spalte auf und beinhaltet die Copolymerzusammensetzungen wie sie durch die Referenzmessung bestimmt wurden. Die Reihenvektoren der $X$-Matrix werden durch die zugehörigen Absorbanzwerte der (Kalibrierungs)-IR-Spektren bestimmt. Die Spektraldaten werden Punkt für Punkt zeilenweise in die $X$-Matrix geschrieben. Jede weitere Probe entspricht einer neuen Matrixzeile. Ziel ist es, den Vektor $b$ zu bestimmen. Ist einmal die Kalibrierfunktion $K$ ermittelt, kann mit dem erhaltenen Modell später eine Vorhersage für unbekannte Werte von $Y_{\mathrm{n}}$ getroffen werden.

Aus der Literatur ist eine Vielzahl an Regressionsmethoden zur Berechnung der Kalibrierfunktion $K$ bekannt. Eine vergleichende Übersicht findet sich in [18, 19, 20, 21]. Im Rahmen dieser Arbeit wird zur Ermittlung der Kalibration das Programmpaket Quant 2 für Opus (Bruker Optik GmbH, Karslruhe) verwendet. Diese Software nutzt zur Berechnung der Kalibrierfunktion den von Wold [22] entwickelten PLS-Algorithmus (partial least square), welcher sich in der Praxis als die bewährteste Methode etabliert hat. Grundidee ist hierbei, die Spektraldatenmatrix und die Eigenschaftsdatenmatrix $Y$ auf nur wenige Faktoren $\mathrm{zu}$ reduzieren. Anschließend wird dasjenige Kalibrationsmodell gesucht, welches mit der geringsten Anzahl an Faktoren (auch als Rang bezeichnet) möglichst optimal die Spektraldaten beschreibt. Somit wird vermieden, dass das Modell auch Feinstrukturen wie z.B. spektrales Rauschen wiedergibt und damit unnötig kompliziert wird. Aufgrund des Umfangs und der mathematischen Komplexität wird hier auf eine Erläuterung des PLSVerfahrens im Detail verzichtet. Ausführliche Informationen über das PLS-Verfahren und dessen verschiedene Varianten sind in [19, 20] dokumentiert. 
Die Methode der multivariaten Kalibration weist gegenüber der univariaten Variante eine Reihe von Vorteilen auf:

1. Die Unsicherheit der Vorhersage wird bei der multivariaten Kalibration nicht direkt durch statistische Signalschwankungen, wie z.B. Detektorrauschen, beeinflusst. Derartige Schwankungen sind im Fall der univariaten Methode besonders dann problematisch, wenn nur eine Wellenlänge oder ein kleiner Wellenlängenbereich zur Auswertung verwendet wird.

2. Im Unterschied zur univariaten Methode ist die multivariate Kalibrierung nicht auf Fälle beschränkt, in denen für jede Komponente hinreichend getrennte Signale beobachtet werden. Es können auch stark überlappende Banden für die Auswertung verwendet werden, solange sich diese in ihrer Struktur zumindest geringfügig unterscheiden. Die Methode ist daher für die Analyse auch komplexer Mehrkomponentensysteme geeignet.

3. Bei der univariaten Kalibration wird bei der Analyse von Mehrkomponentensystemen die lineare Additivität der Extinktionswerte aller Komponenten bei den betrachteten Wellenlängen unterstellt. Dies ist nicht in allen Fällen gegeben. Für die multivariate Kalibration werden derartige Abweichungen der Kalibration berücksichtigt und sind daher unproblematisch.

4. Veränderungen des Spektrums durch Verzerrung der Bandenform aufgrund intermolekularer Wechselwirkungen, Temperatur- oder Druckeinflüsse verfälschen die Kalibration nicht, da sie durch geeignete Probenwahl im Analysenmodell mitberücksichtigt werden können.

5. Die multivariate Kalibration ist nicht beschränkt auf spektroskopische Methoden für welche das Lambert-Beersche Gesetz gilt und kann daher auch für Reflexionstechniken wie ATR-Messungen [23] u.a. eingesetzt werden.

6. Im Unterschied zur univariaten Kalibrierung können beim multivariaten Verfahren Ausreißer oder Störungen aufgrund unbekannter Komponenten leicht anhand der Analyse des Residuums eines Analysenspektrums oder der Mahalanobis-Distanz [24] (Differenz aus dem tatsächlichen Spektrum und dem Mittelwert aller Spektren des Kalibrationssatzes) erkannt werden. 
Die voranstehenden Vorteile sollten die multivariate Kalibration in vielen Fällen zur überlegenen Methoden machen. Im Rahmen dieser Arbeit sollen am Beispiel der IR-NMRKorrelation für die hier untersuchten Copolymersysteme die beiden Methoden bezüglich ihrer Vorhersagegenauigkeit verglichen werden. Insbesondere erscheint es interessant $\mathrm{zu}$ prüfen, inwieweit sich die Auswertung für das Copolymersystem E-MMA, bei dem nach der univariaten Methode aufgrund überlappender Banden eine Bandenanalyse nötig ist, vereinfachen und die Präzision verbessern lässt.

Ein wichtiger Aspekt bei der multivariaten Analyse ist die Vorbehandlung der Daten. In der quantitativen Analyse wird vorausgesetzt, dass alle Messungen bei gleicher Schichtdicke ausgeführt wurden. Gerade für die im Rahmen dieser Arbeit aufgenommenen Folienspektren ist eine Normierung unumgänglich, da es mit der verwendeten Technik nicht möglich ist, Folien mit exakt gleicher Stärke herzustellen. Zur Optimierung des Kalibrationsprozesses wurden folgende Datenvorbehandlungsmethoden auf ihre Eignung getestet:

1. Eliminierung eines konstanten Offsets.

2. Subtraktion einer Geraden (SG):

Hierzu wird eine Gerade an die Grundlinie des Spektrums angepasst und anschließend subtrahiert.

3. Vektornormierung $(\mathrm{VN})$ :

Das Spektrum wird zunächst durch Subtraktion des mittleren Y-Werts zentriert. Anschließend wird die Summe der Quadrate aller Werte berechnet und das Spektrum durch die Wurzel der vorgenannten Summe dividiert.

4. Min-Max-Normierung (MMN):

Es wird der konstante Offset subtrahiert und das resultierende Spektrum mit einer Konstanten derart multipliziert, dass der maximale Wert bei 2 Absorptionseinheiten liegt.

5. Multiplikative Streukorrektur (MS):

Für jedes Spektrum wird eine lineare Transformation durchgeführt, so dass es dem Mittelwert aller Spektren am nächsten kommt.

6. Erste bzw. zweite Ableitung (1A, 2A):

Es wird die erste bzw. zweite Ableitung der Spektren berechnet. Dies betont besonders steile Peakflanken und verbessert die Analyse kleiner Veränderungen vor einem breiten Hintergrund. 
Es sei vorweggenommen, dass sich im Rahmen der für die verschiedenen Copolymersysteme durchgeführten Optimierungen überwiegend die Methode der multiplikativen Streukorrektur als besonders geeignet erwiesen hat. Dennoch erscheint es sinnvoll, prinzipiell die verschiedenen Vorbehandlungsmethoden für jede einzelne Kalibrieraufgabe $\mathrm{zu}$ testen, da eine Vorhersage bezüglich der Eignung verschiedener Vorbehandlungsmethoden kaum möglich ist.

Zur Validierung eines ermittelten Kalibrationsmodell, d.h. zum Test der Leistungsfähigkeit der späteren Voraussage, können bei Anwendung des Quant 2-Programmes zwei Methoden gewählt werden. Bei der Test-Set-Validierung (externe Validierung) wird lediglich ein Probensatz für die Kalibration und ein zweiter zur Validierung des ermittelten Modells verwendet. Bei der im Rahmen dieser Arbeit gewählten Kreuzvalidierung (interne Kalibrierung) wird ein und derselbe Datensatz zu Kalibration und Validierung verwendet. Bei diesem Verfahren wird während der Modellerstellung jeweils eine Probe weggelassen, anhand der das Modell getestet werden kann. Anschließend wird der Vorgang mit einer anderen Probe durchlaufen und wiederholt bis alle Proben einmal zu Validierung herangezogen worden sind. Der Vorteil dieser Methode besteht darin, dass mit einem erheblich kleineren Satz Proben gearbeitet werden kann

Um mit dem Programm Quant 2 die jeweils beste Kalibration für ein Copolymersystem zu ermitteln, wurde wie folgt vorgegangen: Zunächst wurde für den jeweiligen Spektrensatz ein automatischer Testlauf von Kreuzvalidierungen durchgeführt. Dazu wurde der Spektralbereich der Spektren auf die Bereiche beschränkt, in denen ein lineares Verhalten des IR-Detektors gewährleistet ist (maximaler Absorbanzwert kleiner 1.6). Bei diesem Testlauf werden sukzessive Kombinationen von vordefinierten Frequenzbereichen und Datenvorbehandlungsmethoden (s.o) berechnet. Mit der so ermittelten geeignetsten Methode wurde nachfolgend anhand nochmals einzeln ausgeführter Rechnungen unter Variation der Spektralbereiche die optimale Validierung gefunden.

Zur Beurteilung der Güte einer Kalibration kann der mittlere Vorhersagefehler RMSEP (ㅁoot mean square error of prediction) dienen. Er berechnet sich aus der Summe der quadratischen Abweichungen des Vorhersagewertes zum wahren Wert wie folgt:

$$
R M S E P=\sqrt{\frac{1}{N} \sum_{\mathrm{i}=1}^{N}\left(Y_{\mathrm{i}}^{N M R}-Y_{\mathrm{i}}^{\text {Vorhersage }}\right)^{2}}
$$


$N$ bezeichnet hierbei die verwendete Anzahl Kalibrationsproben. Die Details der für die verschiedenen Copolymersysteme mit dem Programm Quant 2 ermittelten optimalen Kalibrierungen sind in Tabelle 3.8 aufgelistet. Angeführt ist der ermittelte Rang, die Datenvorbehandlungsmethode (abgekürzt), der erzielte RMSEP-Wert und der beobachtete maximale Vorhersagefehler. Zum Vergleich der beiden Methoden wurde nach Gl. 3.22 RMSEP-Werte auch für die Vorhersagewerte der univariaten Methode berechnet und in Tabelle 3.8 mit aufgenommen.

\begin{tabular}{ccccccc}
\hline \hline Copolymer & $N$ & $\begin{array}{c}\text { Wellenzahlbereich } \\
\text { Quant 2 }\left[\mathrm{cm}^{-1}\right]\end{array}$ & Rang & $\begin{array}{c}\text { Datenvor- } \\
\text { behandlung }\end{array}$ & $\begin{array}{c}R M S E P \text { multivariat/ } \\
\text { Max. Fehler [mol-\%] }\end{array}$ & $\begin{array}{c}R M S E P \text { univariat/ } \\
\text { Max. Fehler [mol-\%] }\end{array}$ \\
\hline \hline E-EA & 28 & $\begin{array}{c}6034.3-3301.6 \\
2729.8-2446.3\end{array}$ & 4 & MMN & $0.49 / 1.1$ & $0.65 / 1.5$ \\
E-PA & 21 & $\begin{array}{l}4543.6-3356.6 \\
2738.5-2468.5\end{array}$ & 5 & MS & $0.63 / 1.29$ & $0.66 / 1.84$ \\
E-MMA & 21 & $6289.9-4221.8$ & 2 & MS & $0.74 / 1.31$ & $1.55 / 3.40$ \\
E-EMA & 17 & $6043.9-3373.9$ & 3 & VN & $0.90 / 1.52$ & $1.32 / 2.71$ \\
E-BzMA & 7 & $6082.0-3321.7$ & 3 & MS & $0.70 / 0.95$ & $0.83 / 1.64$ \\
\hline
\end{tabular}

Tabelle 3.8: Aufstellung der für die optimale Kalibration verschiedener Copolymersysteme genutzten Wellenzahlbereiche, der verwandte Rang und der Zahl der Proben, sowie der erzielte RMSEP-Wert. Der für die univariate Methode erhaltene RMSEP-Wert ist zum Vergleich ebenfalls angegeben.

Aus Tabelle 3.8 ist klar ersichtlich, dass die Methode der multivariaten Kalibration sehr gut für die hier betrachtete Kalibrationsaufgabe geeignet ist. Es werden für alle Copolymersysteme bei vergleichsweise niedriger Komplexität der Modelle, erkennbar an den kleinen Rangwerten, geringe Vorhersagefehler erzielt. Im Vergleich zeigt sich, dass die Methode der multivariaten Korrelation für alle Copolymersysteme präzisere Vorhersagen ermöglicht. Der mittlere Vorhersagefehler als auch die maximale Abweichung fallen signifikant niedriger aus als bei der univariaten Korrelation. Besonders deutlich wird dies - wie zu erwarten - für das System Ethen-MMA. So lässt sich mit der multivariaten Methode hier eine mehr als doppelt so präzise Vorhersage der Copolymerzusammensetzung erreichen. 
Zusammenfassend lässt sich festhalten, dass die Analyse der Zusammensetzung von Ethen(Meth)Acrylat-Copolymeren anhand von IR-Filmspektren in guter Genauigkeit möglich ist. Für die Aufstellung der hierzu notwendigen NIR/IR-NMR-Korrelationen wurde eine uniund eine multivariate Methode vorgestellt. Die Ergebnisse zeigen, dass die multivariate Methode zu bevorzugen ist, da diese im Vergleich präzisere Vorhersagen ermöglicht und universell, d.h. auch auf Systeme mit überlappenden IR-Banden wie z.B. E-MMA, anwendbar ist. Ein weiterer Vorteil der multivariaten Analyse besteht darin, dass mit der Berechnungsmethodik Verfälschungen durch Probenverunreinigungen oder Ausreißer leicht erkannt werden können.

\subsubsection{Bestimmung der Molekulargewichtsverteilung}

Ethen-(Meth)Acrylsäureester-Copolymere bestehen aus Makromolekülen mit unterschiedlichem Molekulargewicht. Die Molekulargewichtsverteilung beschreibt die Häufigkeit mit der Moleküle eines bestimmten Molekulargewichts vorkommen. Diese hat in der Regel einen Einfluss auf die physikalischen Eigenschaften des Polymers als auch auf das Phasenverhalten entsprechender Solvens-Polymer-Systeme [25, 26, 27].

Die Standardmethode zur Bestimmung der Molekulargewichtsverteilung von Polymeren ist die Gelpermeationschromatographie (GPC). Es handelt sich dabei um eine FlüssigkeitsSäulenchromatographie, bei der eine Lösung der Makromoleküle mit konstanter Geschwindigkeit durch eine oder mehrere Trennsäulen gepresst wird, welche mit einem mikroporösen Polymergel gefüllt sind. Die Trennwirkung beruht auf der Diffusion der Makromoleküle zwischen mobiler Phase und den Poren des Gels. Kleineren Molekülen sind eine größere Anzahl von Poren zugänglich. Folglich werden kleinere Moleküle am stärksten retendiert und eluieren später als höhermolekulare Fraktionen. Es findet somit eine Trennung nach dem hydrodynamischen Volumen der Polymermoleküle statt. Am Säulenausgang wird die jeweils austretende Polymerkonzentration beispielweise mittels eines Refraktrometers oder UV-spektroskopisch detektiert.

Die Retentionszeiten hängen stark von den Messbedingungen (Polymerart, Säulenmaterial, Lösungsmittel) ab und haben keinen direkten Bezug zum Molekulargewicht des untersuchten Polymers. Absolutwerte des Molekulargewichts können deshalb nur nach Eichung über Polymerstandards mit bekannter Molekulargewichtsverteilung erhalten werden. Für die hier untersuchten Copolymersysteme stehen Standards bisher nicht zur Verfügung. Eine andere Möglichkeit zur Bestimmung absoluter Molmassen ist das Prinzip der universellen 
Eichung unter Verwendung von Mark-Houwink-Koeffizienten [28]. Derartige Koeffizienten stehen jedoch bisher ebenfalls für keines der im Rahmen dieser Arbeit untersuchten Copolymersysteme zur Verfügung. Die einzig bekannten Daten existieren für EthenMethylacrylat-Copolymere [29]. Im Rahmen dieser Arbeit wurden daher die Molekulargewichte von Ethen-Copolymeren der Methyl-, Ethyl- und Butylester der (Meth)Acrylsäure unter Anwendung dieses Koeffizientensatzes bestimmt. Die ebenfalls untersuchten EBzMA-Copolymere wurde aufgrund deren engeren chemischen Verwandtschaft mit einem Polystyrolstandard ausgewertet. Für die Molekulargewichtsbestimmung von Poly(meth)acrylaten wurden Mark-Houwink-Koeffizienten aus der Literatur genutzt [30, 31]

Die Mittelwerte des Molekulargewichts sind wie folgt definiert:

Zahlenmittel des Molekulargewichts

$$
M_{\mathrm{n}}=\frac{\sum_{i} n_{i} \cdot M_{i}}{\sum_{i} n_{i}}
$$

Gewichtsmittel des Molekulargewichts

$$
M_{\mathrm{w}}=\frac{\sum_{i} m_{i} \cdot M_{i}}{\sum_{i} m_{i}}=\frac{\sum_{i} n_{i} \cdot M_{i}^{2}}{\sum n_{i} \cdot M_{i}}
$$

Hierbei entsprechen $n_{i}, m_{i}$ und $M_{i}$ der Stoffmenge, der Masse und dem Molekulargewicht eines makromolekularen Teilchens $i$. Die Breite einer Molekulargewichtsverteilung ist durch das Verhältnis zwischen $M_{\mathrm{n}}$ und $M_{\mathrm{w}}$ charakterisiert. Die Polydispersität $Q$ wird durch den Quotienten aus Gewichtsmittel und Zahlenmittel definiert. $Q$ ist immer $\geq 1$.

Eine Aufstellung der im Rahmen dieser Arbeit untersuchten Copolymere mit dem jeweiligen Methacrylatgehalt und dem Zahlen- und Gewichtsmittel des Molekulargewichts findet sich in Anhang B. Die dort mitaufgeführten Polyethylenproben sind ebenfalls Produkte von Synthesen in dem in Abschnitt 3.1 beschriebenen Rührkessel.

Die im Rahmen dieser Arbeit ermittelten Molekulargewichtsverteilungen wurden auf einem System bestehend aus HPLC-Pumpe (Waters, Modell 515), Trennsäule (PSS-SDV) und Differentialrefraktometer (Waters, Modell 2410) als Detektor gemessen. Die Polymerkonzentration betrug $3 \mathrm{mg} \mathrm{ml}^{-1}$. Die Trennsäule wurde auf $35^{\circ} \mathrm{C}$ thermostatisiert. Als Eluent wurde Tetrahydrofuran (THF, Fluka puriss. p.a.) bei einer Flussrate von $1 \mathrm{ml} \mathrm{min.} .^{-1}$ verwendet. 
Allgemein besteht für Ethen-Copolymere das Problem schlechter Löslichkeit in THF bei $35^{\circ} \mathrm{C}$. Typischerweise können unter diesen Bedingungen nur Proben mit einem (Meth)Acrylatgehalt größer als ca. 13 mol-\% vollständig gelöst und mit der GPC vermessen werden. Es ist anzumerken, dass die Löslichkeitsprobleme durch Vermessung der Proben mit einer Hochtemperatur-GPC-Anlage vermieden werden können. In dieser Arbeit stand eine Hochtemperatur-GPC allerdings nicht zur Verfügung.

\subsection{Trübungsdruckmessung an Ethen / Polymer-Systemen}

\subsubsection{Verwendete Substanzen}

Die bei der Messung von Trübungsdrücken verwendeten Chemikalien sind mit dem vom Hersteller angegebenen Reinheitsgrad in Tabelle 3.9 aufgelistet. Die Chemikalien wurden ohne weitere Reinigung in den Experimenten eingesetzt.

\begin{tabular}{|c|c|c|c|}
\hline & Chemikalie & Reinheitsgrad & Hersteller \\
\hline \multirow[t]{2}{*}{ a) } & Ethen & $\mathrm{O}_{2}$-Gehalt $\leq 5 \mathrm{ppm}$ & BASF AG \\
\hline & Propen & mind. $99.8 \%$ & BASF AG \\
\hline \multicolumn{2}{|c|}{$\begin{array}{l}\text { 2,6-Di-tert-butyl-4-methylphenol } \\
\text { (DtBMP) }\end{array}$} & $>99 \%$ & Merck-Schuchardt \\
\hline & $n$-Heptan & mind. $99 \%$ & Merck-Schuchardt \\
\hline b) & Kohlendioxid & 4.5 & Messer-Griesheim \\
\hline \multirow[t]{3}{*}{ c) } & Methylcyclohexan & mind. $98 \%$ & Fluka AG \\
\hline & iso-Dodekan & mind. $98 \%$ & BASF AG \\
\hline & Aceton & $99.5 \%$ & Riedel-de-Haën \\
\hline
\end{tabular}

Tabelle 3.9: Verwendete Chemikalien und der vom Hersteller angegebene Reinheitsgrad. Anwendung: a) Trübungspunktmessung, b) Dichtigkeitstest, c) Reinigung. 


\subsubsection{Experimentelle Anordnung für diskontinuierliche Messungen}

Der überwiegende Teil der Trübungsdruckmessungen von Ethen-Copolymer-Mischungen wurde diskontinuierlich in einer Satzzelle durchgeführt. Die dazu verwendete Apparatur besteht aus einer Hochdruckzelle mit Anbauteilen, einer Druckerzeugungseinrichtung und einer Endoskopoptik. Im folgenden Abschnitt wird zunächst die Hochdruckzelle als Kernstück des experimentellen Aufbaus detailliert beschrieben. Anschließend findet sich eine Erläuterung des Gesamtaufbaus.

\section{Hochdruckzelle}

Die Konstruktion der verwendeten Zelle (Abb. 3.10) leitet sich von einer von Buback [32] beschriebenen Hochdruck-Durchstrahlzelle ab. Der hohlzylindrische Zellkörper (3) besteht aus einer hochwarmfesten Nickelbasislegierung (RGT 601, Werkstoff-Nr. 2.4668, ArbedSaarstahl). Er hat eine Länge von $171 \mathrm{~mm}$, einen Außendurchmesser von $80 \mathrm{~mm}$ und einen Innendurchmesser von $22 \mathrm{~mm}$. Die hohe Zugfestigkeit des Werkstoffs ( $\geq 1080 \mathrm{~N} \cdot \mathrm{mm}^{-2}$ bis $500{ }^{\circ} \mathrm{C}$ ) erlaubt unter Berücksichtigung eines Sicherheitsbeiwertes von 2.5 bei dem gewählten Verhältnis von Außen- zu Innendurchmesser die Verwendung der Zelle bis zu einem Druck von 3000 bar bei $300^{\circ} \mathrm{C}$.

Die Dichtung erfolgt durch konische Stempel, welche durch Flansche (1) (RGT 601) mit jeweils sechs Schrauben (7) (RGT 12, Werkstoff-Nr. 2.4969, Arbed-Saarstahl) an den Zellkörper gepresst werden. Die Schrauben werden dazu mit einem Drehmoment von jeweils $30 \mathrm{Nm}$ angezogen. Die Öffnungswinkel der Konen betragen $78^{\circ}$, die der Stempel $75^{\circ}$, so dass die Flächen der Stempel gegen die Innenkanten des Zellkörpers dichten. Die Stempel (ebenfalls RGT 12) bestehen aus weicherem Material als der Zellkörper. Somit tritt Verschleiß in Form sich einprägender Dichtlinien vornehmlich an den Stempeln auf. Dies erleichtert eine eventuell notwendig werdende Überarbeitung.

Einer der beiden Stempel (6) besitzt zur Beobachtung des Phasengeschehens eine zentrale Bohrung von $10 \mathrm{~mm}$ Durchmesser. Das darauf mit einer Überwurfkappe (4) (Edelstahl, Werkstoff-Nr. 1.4122) fixierte Fenster (8) (Durchmesser: 18 mm, Höhe: $10 \mathrm{~mm}$ ) besteht aus synthetischem Saphireinkristall (UV-grade, Roditi, Union Carbide). Zur Verringerung von Lichtreflexion und Streuverlusten ist das Fenster beiderseits optisch eben poliert. Zum Ausgleich von Oberflächenunebenheiten befindet sich zwischen der mit Diamantpaste der Körnung 4-8 $\mu \mathrm{m}$ polierten Auflagefläche des Stempels und dem Fenster eine $12 \mu \mathrm{m}$ dünne Teflonfolie. Diese auf dem Prinzip von Poulter [33] beruhende Art der Dichtung nimmt in 
ihrer Wirksamkeit mit dem Druck zu. Der andere drucktragende Stempel (11) ist mit einer Verschraubung zum Anschluss einer Hochdruckkapillare (Innen 1/16 Zoll, Außen 1/4 Zoll, Autoclave Engineers) versehen und wird so mit der Druckerzeugungseinrichtung (siehe Abb. 3.11) verbunden.

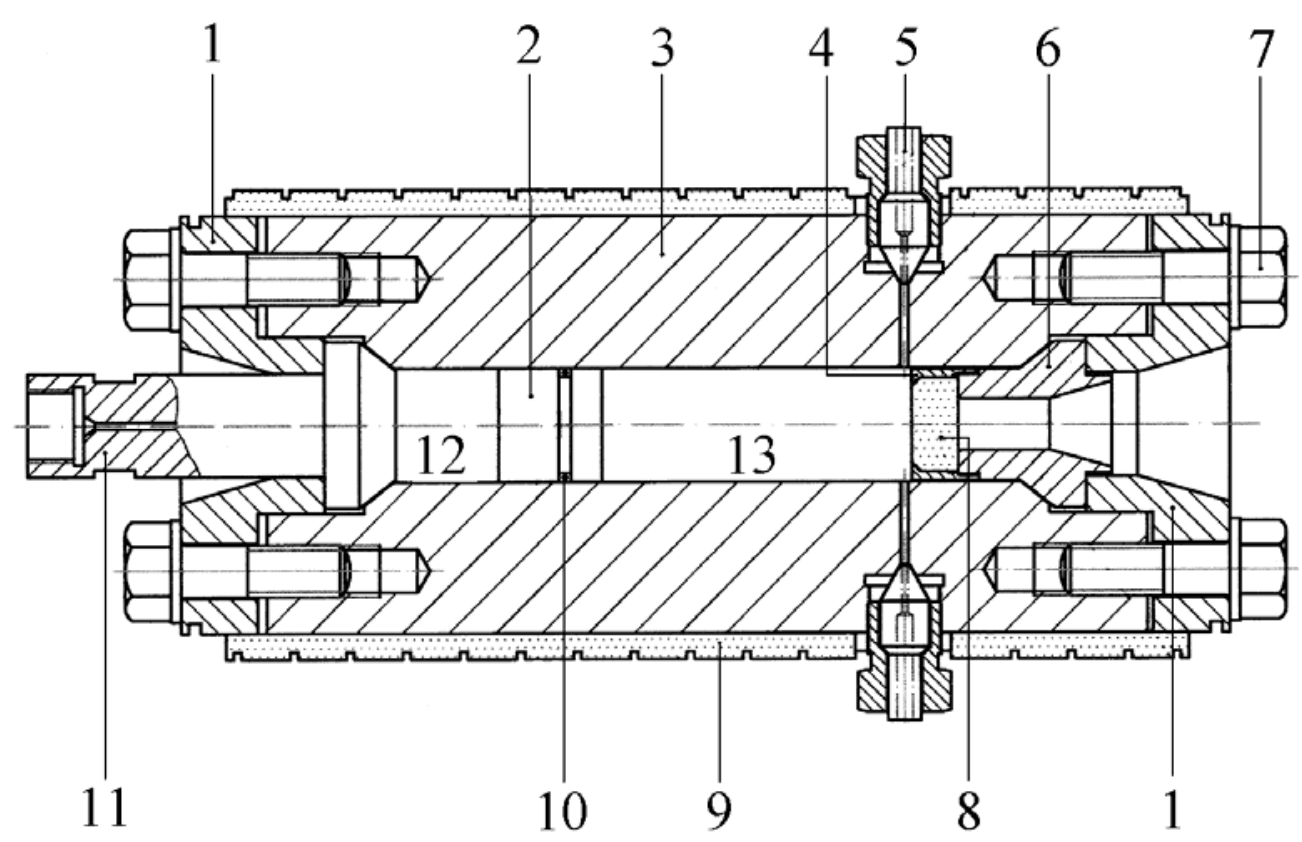

Abbildung 3.10: Schnittzeichnung der Hochdruckzelle zur Trübungspunktmessung

(1) Flansch, (2) Gleitkolben, (3) Zellkörper, (4) Überwurfkappe, (5) Mantelthermoelement, (6) Fensterstempel, (7) Schraube, (8) Saphirfenster, (9) Messingmatrix mit Heizleitern, (10) O-Ring-Dichtung, (11) Stempel mit Kapillarverschraubung.

In der Trübungspunktmesszelle befinden sich auf Höhe der Stirnseite des Saphirfensters jeweils im Winkel von $90^{\circ} \mathrm{C}$ vier radiale Bohrungen. Diese dienen zum Anschluss einer Hochdruckkapillare, einer Berstscheibenhalterung und zum Einführen zweier Mantelthermoelemente (5) (Chromel-Alumel, CIA S250, CGE Alsthom). Die Thermoelemente sind dazu jeweils in einem Blindkonus hart eingelötet. Ein Element misst die Temperatur im Mantel der Zelle und dient in Kombination mit einem PID-Regler (Eurotherm 815) zur Temperierung der Zelle. Die Fühlerspitze des anderen Thermoelements ragt bis in den Probenraum, so dass zusätzlich direkt in der Polymer-Solvens-Mischung gemessen werden kann. Die Zelle wird mit Widerstandsmantelheizleitern (Pyrolon-M16, $16 \Omega \mathrm{m}^{-1}$, Les Cables de Lyon) geheizt, die in einer über die Zelle geschobenen, zweiteiligen Messingmatrix (9) eingelassen sind. Die Heizleistung wird mit einem PID-Regler (Eurotherm Typ 815) 
gesteuert. Durch einen O-Ring-gedichteten Gleitkolben (2) (Länge $20.1 \mathrm{~mm}, \varnothing 21.9 \mathrm{~mm}$, Werkstoff-Nr. 1.4122), dessen Stirnseite zum Saphirfenster zur besseren Reflexion des eingestrahlten Lichts poliert ist, wird der Innenraum der Zelle in einen Probenraum (13) und einen Raum für das Druckmittel $n$-Heptan (12) geteilt.

Zur Dichtung des Gleitkolben wurden O-Ringe aus Kalrez ${ }^{\circledR}$ (Compound 4079, Du Pont) [34] gewählt. Dieses Material ist vom Hersteller für Temperaturen bis $316^{\circ} \mathrm{C}$ (unter Luftatmosphäre) spezifiziert. Die O-Ringe aus Kalrez ${ }^{\circledR}$ erwiesen sich unter den Bedingungen der Trübungspunktmessungen der vorliegenden Arbeit, d. h. bei Temperaturen bis $260{ }^{\circ} \mathrm{C}$ und Anwesenheit von hochverdichtetem Ethen bzw. Propen, auch über längere Zeit (24 h) als stabil.

\section{Gesamtaufbau}

Der Gesamtaufbau der Apparatur zur Bestimmung von Trübungsdrücken ist in Abbildung 3.11 schematisch dargestellt.

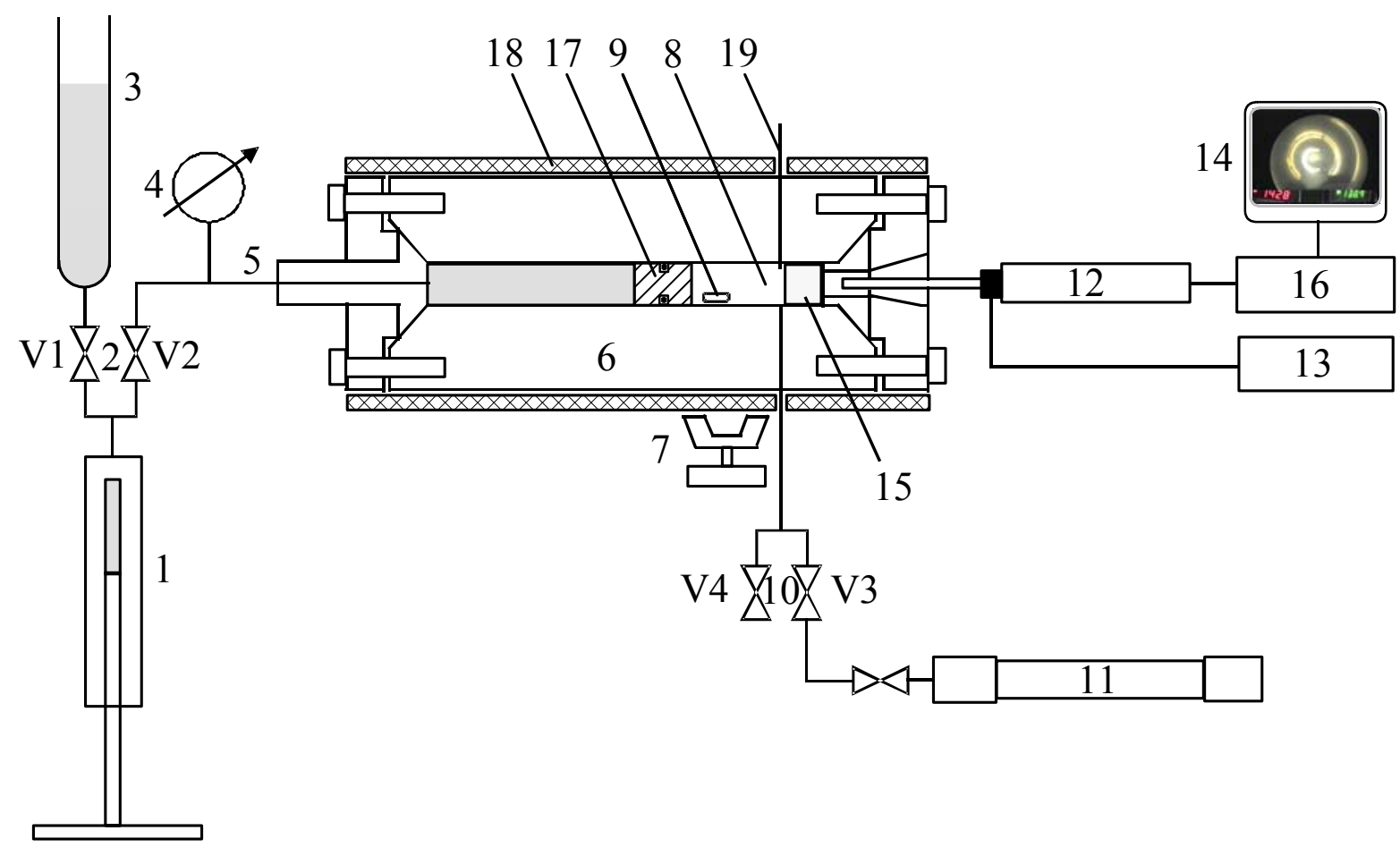

Abbildung 3.11: Apparatur zur Bestimmung von Trübungspunkten (schematisch)

(1) Spindelpresse, (2) Doppelspindelventil, (3) Druckmittelvorratsgefä $\beta$, (4) Druckaufnehmer, (5) Anschlusskapillare, (6) Hochdruckzelle, (7) Magnetrührer, (8) Probenraum, (9) Magnetrührstäbchen, (10) Doppelspindelventil, (11) Füllautoklav, (12) Endoskopkamera, (13) Halogenlichtquelle, (14) Monitor, (15) Saphirfenster, (16) Videorekorder, (17) Gleitkolben mit O-Ring-Dichtung, (18) Heizschale mit Heizleitern, (19) Thermoelement. 
Die Druckerzeugungseinrichtung besteht aus einer Spindelpresse (1) (Innenvolumen $12 \mathrm{~cm}^{3}$ ), welche mittels Kapillaren über ein Doppelspindelventil (2, V1/V2) mit einem Vorratsgefäß (3) für das Druckmittel (über V1) und dem Druckaufnehmer (4) (über V2) sowie über eine Anschlusskapillare (5) mit der Trübungspunktmesszelle (6) verbunden ist. Zur Druckmessung wird ein DMS Druck-Umwandler (3 kbar, Klasse 0.2, HBM-Messtechnik) eingesetzt. Das Gerät ist gegen ein Präzisionsmanometer (Klasse 0.05, Wika) als Referenz kalibriert. Mit einem motorgetriebenen Hufeisenmagneten (7) läßt sich im Probenraum (8) der Zelle ein teflonummanteltes achtkantiges Magnetrührstäbchen mit Mittelring (9) $\left(16 \times 8 \mathrm{~mm}, \mathrm{Neolab}^{\circledR}\right)$ bewegen und so eine gute Durchmischung des Polymeren mit dem Lösungsmittel erreichen.

Der Probenraum ist über eine Kapillare mit einem Doppelspindelventil (10) (V3/V4) verbunden. Über dieses kann die Zelle (über V3) mittels eines mit einer Berstscheibe (200 bar, Fa. Rembe, Brilon) gesicherten Füllautoklaven (11) mit Ethen befüllt werden.

Die Eintrübung bzw. Homogenität der zu untersuchenden Mischung wird mit einem Endoskop (12) (Optikon) mit CCD-Kamera und Halogenkaltlichtquelle (13) (Techno 150 CD, Optikon) und daran angeschlossenem Monitor (14) durch das Saphirfenster (15) beobachtet. Zusätzlich können die Druck- und Temperaturanzeigen, die von einer zweiten Kamera (C-MOS-Modul) aufgenommen werden, mit einer Video-Mischeinrichtung (MX-1, Videonics) in das Bild der Endoskopkamera eingespiegelt und zur Dokumentation des Experiments von einem S-VHS-Videorekorder (16) aufgezeichnet werden. Um eine Beschädigung des Endoskops durch Überschreiten der maximalen Betriebstemperatur von $140{ }^{\circ} \mathrm{C} \mathrm{zu}$ verhindern, wird es auf einer Halterung in einem Messingrohr fixiert und von durchströmender Pressluft gekühlt.

Die oben beschriebene Apparatur birgt das Risiko von Druckspitzen, welche den maximal zulässigen Betriebsdruck von 3 kbar überschreiten könnten. Sie können durch die Wärmeentwicklung einer möglicherweise trotz Inhibition ablaufenden Polymerisationsreaktion, durch eine spontane Zersetzungsreaktion des Ethens [35] oder auch durch Überheizen aufgrund eines Defekts der Heizungsregelung hervorgerufen werden. Zur Verringerung der resultierenden Gefahr ist zusätzlich zur selbstentlastenden Wirkung des zur Dichtung des Autoklaven angewandten Konenprinzips an einer der Bohrungen des Zellkörpers eine Berstscheibe (Berstdruck 3114 bar, M-5908-21, OSECO, Broken Arrow, USA) in einer entsprechenden Halterung (CS 4600, 1/4 Zoll, Autoclave Engineers) angebracht. 
Die Apparatur ist zudem mit an Stahlprofilen verschraubten, starken Plexiglasplatten umbaut, um auch die Gefahr durch versagende Hochdruckbauteile (z.B. Splitter eines berstenden Saphirfensters, einer ansprechenden Berstscheibe) zu mindern.

\subsubsection{Durchführung von Trübungdruckmessungen mit der Satzzelle}

Nach dem Verschrauben des fenstertragenden Stempels, der Thermoelemente und der Berstscheibenhalterung mit dem Zellkörper werden typischerweise etwa $440 \mathrm{mg}$ Polymer und $40 \mathrm{mg}$ Inhibitor (DtBMP) in die Zelle eingewogen sowie das Magnetrührstäbchen hinzugegeben. Anschließend drückt man den mit einem neuen O-Ring versehenen Gleitkolben gerade soweit in die Zelle, dass der Stempel mit Kapillaranschluß mittels Flansch montiert werden kann.

Durch kurzes Öffnen von Ventil V1 und V2 wird der Druckerzeugungszweig mit $n$-Heptan gespült und über die Anschlusskapillare mit der mit beiden Heizschalen versehenen Zelle verschraubt. Nach Anschluss der Heizkabel und der Thermoelementleitungen wird das Ventil V3/V4 montiert. Um die im Probenraum vorhandene Luft zu entfernen, wird die Zelle bei geschlossenem Ventil V1/V2 fünfzehnmal über Ventil V3 und eine flexible 1/16 Zoll-HPLC-Kapillare mit je etwa 10 bar Ethen aus der Vorratsflasche befüllt und über V4 wieder entleert.

Zum Befüllen der Zelle mit Ethen kondensiert man zunächst in den mindestens 30 Minuten bei $-20{ }^{\circ} \mathrm{C}$ vorgekühlten Füllautoklaven etwa $3 \mathrm{~min}$. lang Ethen ein (ca. $8 \mathrm{~g}$ ). Anschließend wird der Füllautoklav gewogen. Dabei ist darauf zu achten, dass sich auf dem Füllautoklaven kein Kondenswasser befindet. Nach kurzem Spülen der Zuleitung wird das Ethen über Ventil V3 unter Erwärmen des Autoklaven mit einer Heißluftpistole in die Zelle überführt und der Füllautoklav zur Bestimmung der eingesetzten Ethenmenge zurückgewogen.

Mithilfe der Spindelpresse wird durch Einpressen des Druckmittels über V2 der Druck in der Zelle zur Dichtigkeitskontrolle auf mindestens 1.5 kbar erhöht. Es werden dazu etwa 2 3 Spindelpressenfüllungen benötigt. Wenn auch nach 15 min. kein merklicher Druckabfall aufgrund etwaiger Undichtigkeiten $\mathrm{zu}$ beobachten ist, kann mit dem eigentlichen Experiment begonnen werden. Dazu wird der Rührer sowie die Endoskopkühlung angestellt und die Zelle auf die gewünschte Starttemperatur aufgeheizt. Unter visueller Kontrolle des Lösungsvorgangs wird der Druck mit der Spindelpresse soweit erhöht, bis die untersuchte Mischung eine homogene Phase bildet. Durch langsames Absenken des Drucks mit der Spindelpresse wird der Trübungspunkt (siehe Abschnitt 5.1) erreicht. Man notiert Druck und Temperatur und erhöht den Druck, bis die untersuchte Mischung wieder homogen ist. 
Jede Messung wird mindestens zweimal wiederholt. Anschließend kann auf die nächste Temperatur aufgeheizt werden und eine weitere Messung vorgenommen werden. Während des Aufheizens ist der Druck aus Sicherheitsgründen ständig zu kontrollieren. Nach Abschluss der Messreihe schaltet man die Heizung aus und dreht die Spindelpresse ganz zurück, um den Druck soweit wie möglich abzusenken. Anschließend wird die Zelle mit Pressluft auf Zimmertemperatur heruntergekühlt und der Restdruck durch Lösen der Kapillarverschraubung an Ventil V3/V4 abgelassen. Zur Reinigung wird die Zelle vollständig zerlegt. Eine Probe des untersuchten Polymers wird für spätere Analysen (siehe Abschnitt 5.3.1) aufbewahrt.

\subsubsection{Reinigung}

Das teilweise intensive Anhaften der untersuchten Ethen-Methacrylsäure(ester)copolymere nach dem Erstarren auf Metalloberflächen, eine ansonsten anwendungstechnisch interessante Eigenschaft dieser Materialien [36], erschwert die Reinigung der Hochdruckzelle. Die stärker (meth)acrylathaltigen Probenreste lassen sich in der Regel gut mit Aceton entfernen. Als günstige Reinigungsmethode bei Proben mit niedrigem (Meth)Acrylatgehalt erwies es sich, die Polymerreste zunächst mindestens $30 \mathrm{~min}$. in Methylcyclohexan oder Isododekan bei möglichst hoher Temperatur aufquellen zu lassen. Danach können die Reste mit einer zylindrischen Messingdrahtbürste mechanisch entfernt werden. Abschließend spült man die Bauteile der Hochdruckzelle mit Aceton und trocknet mit Pressluft.

\subsubsection{Experimentelle Anordnung für kontinuierliche Messungen}

Neben der Vorgehensweise das Entmischungsverhalten von Ethen-Copolymer in einer Satzzelle zu untersuchen, lassen sich Trübungsdrücke nach Buback et al. [37] auch mit einem kontinuierlichem Verfahren bestimmen. Hierbei wird der zur Synthese verwendeten Anordnung aus Rührkesselreaktor, IR-Zelle und druckhaltendem Feinventil (siehe Abschnitt 3.1.2) ein zweiter Rührkessel nachgeschaltet, in dem die Temperatur und separat mittels eines zweiten Feinventils auch der Druck eingestellt werden können. Somit werden Trübungsdrücke unmittelbar nach der Synthese an der anwendungsnahen ternären Reaktionsmischung bestehend aus Copolymer, Ethen und nicht abreagiertem (Meth)Acrylatmonomer bestimmt. Dies bedeutet, dass die Polymere vor Abscheidung und Aufarbeitung untersucht werden, so dass eventuelle den Trübungsdruck beeinflussende Polymerveränderungen bei diesen Vorgängen entfallen. 
Im Rahmen dieser Arbeit wurde der Syntheseanordnung anstelle des zweiten Rührkessels eine optische Hochdruckzelle [32] nachgeschaltet. Dies hat mehrere Vorteile: Zum einen ermöglicht die geringere Masse der nachgeschalteten Hochdruckzelle einen erheblich schnelleren Wechsel der Temperatur und damit die Aufnahme einer höheren Zahl von Messpunkten während eines Experiments. Darüber hinaus werden Totvolumina, wie sie beim zweiten Rührkessel z.B. im Rührantrieb auftreten, vollständig vermieden. Zudem hat die Polymer-Monomermischung aufgrund des niedrigeren Innenvolumens der Hochdruckzelle im Vergleich zum zweiten Rührkessel eine wesentlich kürzere Verweilzeit, so dass weitere Copolymerisationsreaktionen in dem für die eigentliche Trübungsdruckmessung verwendeten Volumen nur in erheblich geringerem Ausmaß stattfinden können.

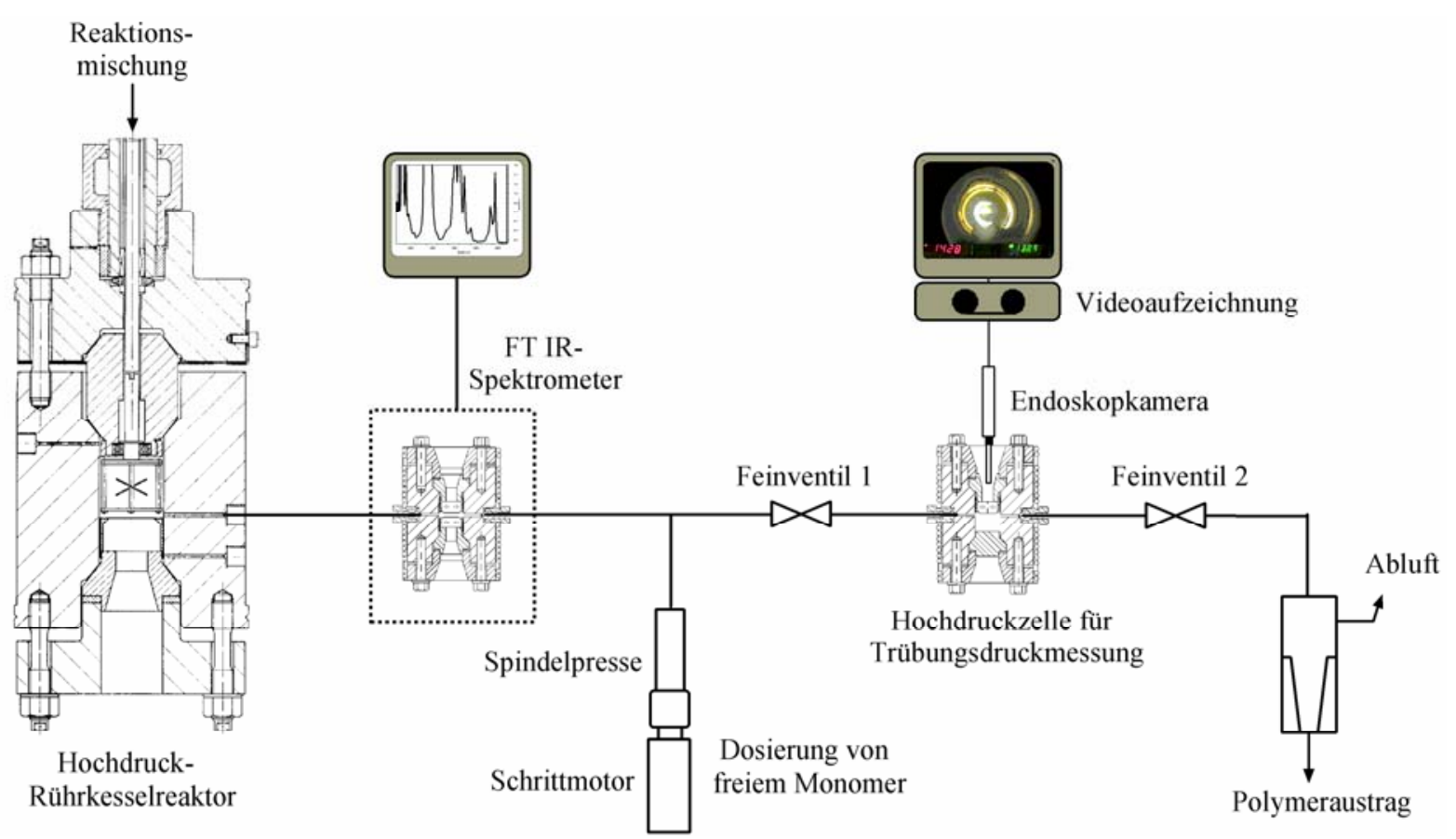

Abbildung 3.12: Experimentelle Anordnung für ,,inline“-Trübungspunktmessungen.

Es sei angemerkt, dass sich durch den einfacheren Aufbau bei gleichzeitig höherer Zuverlässigkeit auch der Wartungsaufwand verringert. In Abbildung 3.12 ist schematisch die für Trübungsdruckmessungen verwendete ,,inline“-Anordnung dargestellt.

Der dem Syntheseaufbau (vgl. Abschnitt 3.1.2) nachgeschaltete Autoklav ist eine Hochdruckdurchstrahlzelle [32], welche mit einem stirnseitig hochglanzpolierten Blindstempel in dem einen und einem Saphirfenster im anderen Flansch ausgerüstet ist. Die Länge des Innenraums beträgt $23.8 \mathrm{~mm}$ bei einem Durchmesser von $22 \mathrm{~mm}$. Mit einer Endoskopkamera (siehe Abschnitt 3.3.2) wird das Geschehen im Innenraum durch das 
Saphirfenster aufgenommen, auf einem Monitor ausgegeben und kann optional auf einem SVHS-Videorekorder aufgezeichnet werden. Durch vorsichtiges Schließen von Feinventil 1 bei gleichzeitigem Öffnen von Feinventil 2 und umgekehrt kann bei konstantem Druck im Synthesereaktor der Druck in der nachgeschalteten Zelle zur Ermittlung des Trübungspunkts variiert werden.

Weiterhin kann bei dem gewählten Synthesedruck mit einer schrittmotorgesteuerten Spindelpresse vor der Zelle zusätzliches Comonomer über das in der Reaktionsmischung enthaltene (Meth)Acrylat hinaus eindosiert werden, um den Einfluss des Comonomergehalts auf den Trübungsdruck zu bestimmen. Die Hochdruckkapillaren zwischen Feinventil 1 und Feinventil 2 sowie die für die Trübungsdruckmessungen verwendete Zelle selbst sind mit Heizleitern ausgerüstet und können mittels PID-Reglern (Eurothern 815) auf die gewünschte Temperatur eingestellt werden. Der maximale Druck für diese Messungen wird durch den Synthesedruck begrenzt.

\subsubsection{Auswahl der Messbedingungen}

Die experimentelle Bestimmung von Trübungspunkten mit dem beschriebenen Aufbau wird grundsätzlich beschränkt durch die Auslegung des Autoklaven auf einen Maximaldruck von 3000 bar bei Temperaturen bis $300^{\circ} \mathrm{C}$. Wie zuvor erwähnt, besteht bei Temperaturen ab ca. $300{ }^{\circ} \mathrm{C}$ zudem die Gefahr der spontanen Zersetzung von Ethen [9].

Der Trübungsdruck von vergleichsweise schwerlöslichen Polymeren, wie z.B. den EthenMethacrylsäure-Copolymeren, steigt mit abnehmender Temperatur sehr stark an. Daher ist die Bestimmung der Trübungsdrücke für diese Proben in Richtung tiefer Temperaturen durch das Erreichen der apparativen Druckobergrenze beschränkt. Bei Experimenten mit Proben von besserer Löslichkeit tritt unterhalb von $100-120^{\circ} \mathrm{C}$ eine Entmischung auf, welche im experimentell zugänglichen Druckbereich nicht reversibel ist. Entsprechend können für diese Proben unterhalb von ca. $100-120^{\circ} \mathrm{C}$ keine Trübungspunkte aufgenommen werden. Es kann vermutet werden, dass in diesem Fall neben den fluiden Phasen auch eine feste Polymerphase auftritt. 


\subsection{Fehlerbetrachtung}

\subsubsection{Unsicherheit der Messgeräte}

- Die Regelgenauigkeit des eingesetzten Massenflussreglers ist nach Herstellerangabe besser als $\pm 0.02 \mathrm{~kg} / \mathrm{h}$ bei einer Reproduzierbarkeit von $1 \%$ des Endwertes. Der Einsatzbereich des Reglers erstreckt sich bis $2 \mathrm{~kg} / \mathrm{h}$.

- Der Fehler der zur Druckmessung verwendeten Absolutdruckaufnehmer (HBMMesstechnik, Klasse 0.2) beträgt $\pm 0.2 \%$ des Skalenendwertes von 3000 bar. Die resultierende Unsicherheit ist somit \pm 6 bar.

- Die Temperatur in der Trübungspunktmesszelle wird mit einem Mantelthermoelement (Chromel-Alumel) direkt in der untersuchten Mischung gemessen. Daher braucht der mögliche Fehler eines Temperaturgradienten zwischen Hochdruckzellenwand und Mischung nicht berücksichtigt zu werden. Die Unsicherheit der Temperaturbestimmung wird vom Hersteller der Thermoelemente mit $\pm 0.25 \mathrm{~K}$ angegeben. Die Genauigkeit der Temperaturbestimmung mit dem verwendeten Thermoelement wurde regelmäßig mit einem kalibrierten Präzisionswiderstandsthermometer (Pt 100, Modell P500, Dostmann) überprüft. Im experimentell genutzten Temperaturbereich betrug die Abweichung der Thermoelemente von der Referenz maximal $0.5 \mathrm{~K}$.

- Die bei der Einwaage des Copolymers (zumeist ca. $440 \mathrm{mg}$ ) auftretende Unsicherheit durch die Ungenauigkeit der Analysenwaage (Satorius, Modell 2401) von $\pm 0.05 \mathrm{mg}$ ist mit $0.011 \%$ vernachlässigbar gegenüber dem Fehler der Einwaage von Ethen. Dieser resultiert aus dem Wägefehler von $\pm 0.01 \mathrm{~g}$ einer Laborwaage (Satorius, Modell BP 1200) und einem geringen Ethenverlust beim Spülen der Zuleitung zu Ventil V3. Der Gesamtfehler der Copolymerkonzentration $\left(w_{\mathrm{p}}\right)$ wird als maximal $\pm 1.0 \%$ abgeschätzt.

- Der Fehler der Copolymerzusammensetzung $\Delta F_{\mathrm{A}}$ resultiert im wesentlichen aus Ungenauigkeiten, welche bei der Auswertung der NMR-Spektren durch die Phasierung, die Basislinienkorrektur und die Integration auftreten. Auf der Basis von Ergebnissen wiederholt ausgeführter Aufnahmen und Auswertungen einzelner Spektren wird die Genauigkeit der NMR-spektroskopisch bestimmten Copolymerzusammensetzung $F_{\mathrm{A}}$ zu \pm 1.3 mol-\% abgeschätzt (vgl. Abschnitt 4.4). 
- Die Unsicherheit der IR-spektroskopischen Copolymeranalyse resultiert aus mehreren Ursachen. Bis zu einer Absorbanz von 1.5 ist ein für quantitative Messungen ausreichend lineares Verhalten des Detektors $\mathrm{zu}$ erwarten. Für Werte zwischen 0.3 und 0.8 Absorbanzeinheiten ist der durch Abweichungen von der Linearität verursachte Fehler des Detektors nach Angabe des Herstellers kleiner als $1 \%$. Bei der Integration können zusätzliche Abweichungen durch Ungenauigkeiten in der Bestimmung der Basislinie auftreten. In Abschnitt 3.2.4 wurde die Unsicherheit der anhand von IR-Kalibrationen bestimmten Copolymerzusammensetzung für die verschiedenen Copolymersysteme geprüft. Der Fehler nach der univariaten Methode ist kleiner als \pm 3 mol- $\%$ und nach der multivariaten Methode kleiner als 1.6 mol-\%.

\subsubsection{Weitere Fehlerquellen}

- Die Unsicherheit des Trübungsdrucks resultiert im wesentlichen aus der visuellen Beobachtung des Phasenverhaltens durch die Ungenauigkeit beim Festlegen des Trübungspunkts (siehe Abschnitt 5.1.2). Das Erkennen des Trübungspunktes während der fortlaufenden Eintrübung beim Absenken des Drucks ist um so schwieriger und damit ungenauer, je weiter der Druckbereich ist, in dem sich die zweite Phase bildet. Mit zunehmender Polydispersität der Polymerproben verbreitert sich der Bereich der Eintrübung [38]. Die im Rahmen dieser Arbeit untersuchten Copolymere haben im Vergleich mit entsprechenden in der Literatur für Trübungspunktmessungen verwendeten Copolymere eine sehr

- geringe Polydispersität. Dies wirkt sich günstig auf die Präzision der Messungen aus. Die Unsicherheit der Trübungsdruckmessung wird auf Basis der Reproduzierbarkeit der Messungen bei tiefen Temperaturen zu kleiner \pm 20 bar abgeschätzt. Mit zunehmender Temperatur nimmt die Unsicherheit bis auf weniger als \pm 10 bar ab.

- Die Bestimmung des Druckes in der Mischung erfolgt indirekt durch Messung im Druckerzeugungszweig. Dabei können die Drücke durch den Reibungswiderstand des Gleitkolbens, welcher die beiden Volumina trennt, voneinander abweichen. In Arbeiten von McHugh et al. [z.B. 39, 40], bei denen eine ähnliche Messtechnik verwendet wurde, wird dieser eventuelle Fehler durch eine geringe Druckkorrektur der Messergebnisse berücksichtigt. Die im Rahmen der vorliegenden Arbeit verwendete diskontinuierliche Apparatur wurde hinsichtlich dieser möglichen Fehlerquelle bereits untersucht [41]. Im untersuchten Bereich von 500-2500 bar wurde keine Druckdifferenz zwischen Probenraum und Druckmittelzweig beobachtet. 
- Wie zuvor beschrieben wird zur stofflichen Trennung der untersuchten Mischung vom Druckmittel ein O-Ring-gedichteter Gleitkolben verwendet. Es ist zumindest denkbar, dass insbesondere bei hohen Drücken und Temperaturen während des Experiments das Druckmittel $n$-Heptan z.T. über diese Dichtung in den Probenraum gelangt. Die wahrscheinliche Auswirkung wären zu gering bestimmte Trübungsdrücke, da von $n$-Heptan ein Cosolvenseffekt zu erwarten ist [42]. Es finden sich unter Berücksichtigung der Messgenauigkeit jedoch keine Anzeichen, dass dieser Effekt eine merkliche Rolle spielt. Zum Ende jeder Messung konnten Trübungsdrücke, welche bereits zu Beginn des Experiments bestimmt wurden, reproduziert werden (siehe Abschnitt 5.3). 


\subsection{Literatur Kapitel 3}

[1] C. Rehberg, Org. Synth. Coll. III (1955) 123

[2] M. Busch, Dissertation, Göttingen (1993)

[3] M. Buback, M. Busch, K. Lovis, F.-O. Mähling, Chem. Ing. Tech. 67 (1995) 1652

[4] M. Buback, M. Busch, K. Lovis, F.-O. Mähling, Macromol. Chem. Phys. 197 (1996) 303

[5] K. Lovis, Dissertation, Göttingen (1995)

[6] L. Wittkowski, Dissertation, Göttingen (1998)

[7] F. O. Mähling, Dissertation, Göttingen (1995)

[8] M. Müller, unveröffentlichte Ergebnisse, Göttingen (2001)

[9] H. Bönsel, G. Luft, Chem. Ing. Tech. 66 (1994) 1170

[10] T. Dröge, Dissertation, Göttingen (1997)

[11] E. F. McCord, W. H. Shaw, Jr., R. A. Hutchinson, Macromolecules 30 (1997) 246

[12] T. H. Black, Aldrichimica Acta 16 (1983) 3

[13] M. D. Lechner, E. H. Nordmeier, Nachr. Chem. Tech. Lab. 42 (1994) 6

[14] L. G. Weyer, Appl. Spectroscopy Rev. 21 (1985) 1

[15] H. D. Siesler, Makromol. Chem. Symp. 52 (1991) 113

[16] M. Buback, M. Busch, T. Dröge, F-.O. Mähling, C. Prellberg, Eur. Polym. J. 33 (1997) 375

[17] P. Antonetti, A. Berrada, M. Chafik El Idrissi, Y. Claire, J. Kaloustian, A. Périchaud, J. Therm. Anal. Calorim. 53 (1998) 923

[18] D. M. Haaland, E. V. Thomas, Anal. Chem. 60 (1998) 1193

[19] K. R. Beebe, B. Kowalski, Anal. Chem. 59 (1987) 1007A

[20] H. Martens, T. Naes, Multivariate Calibration, J. Wiley\&Sons, New York (1989) Kap. 3.5

[21] S. D. Brown, Appl. Spectrosc. 49, No. 12 (1995) 14A

[22] H. Wold, "Partial Least Squares," in S. Kotz and N. L. Johnson (Eds.), Encyclopedia of Statistical Sciences (Vol. 6), J. Wiley\&Sons, New York (1985) 581-591 
[23] N. J. Harrick, Internal Reflectance Spectroscopy, Wiley\&Sons, New York (1967)

[24] M. Otto, Chemometrie; Statistik und Computereinsatz in der Analytik, VCH, Weinheim (1997) 171

[25] S. Lee, M. A. Lostracco, B. M. Hasch, M. A. McHugh, J. Phys. Chem. 98 (1994) 4055

[26] C. J. Gregg, S.-J. Chen, F. P. Stein, M. Radosz, Fluid Phase Equil. 83 (1993) 375

[27] T. W. de Loos, W. Poot, G. A. Diepen, Macromolecules 16 (1983) 111

[28] Z. Grubistic, R. Rempp, H. Benoit, J. Polymer Sci 5 (1967) 753

[29] H.C.M. van Boxtel, Dissertation, Göttingen (2000)

[30] E. Penzel, N. Goetz, Angew. Makromol. Chem. 178 (1990) 191

[31] R. A. Hutchinson, S. Beuermann, D. A. Paquet Jr, J. H. McMinn, Macromolecules 30 (1997) 3490

[32] M. Buback, H. Lendle, Z. Naturforsch. 34 a (1979) 1482

[33] T. C. Poulter, Phys. Rev. 40 (1932) 860

[34] E. I. du Pont de Nemours and Company (1998), http://www.dupont-dow.com/products/index.html

[35] M. Buback, Makromol. Chem. 186 (1980) 373

[36] ExxonMobil (2000),

http://www.exxon.mobil.com/chemicals/cus...es/ldpe_copolymers/hperf_amer_grad e.html

[37] M. Buback, M. Busch, H. Dietzsch, T. Dröge, K. Lovis, Process Technology Proceeedings, "High Pressure Chemical Engineering" (1996) R. v. Rohr, Ch. Tepp (Hrsg.) Elsevier

[38] M. T. Rätzsch, C. Wohlfahrt, D. Browarzik, H. Kehle, J. Macromol. Sci.-Chem. 26 (1989) 1497

[39] S.-H. Lee, M. A. Lostracco, M. A. McHugh, Macromolecules 29 (1996) 1351

[40] B. M. Hasch, M. A. Meilchen, S.-H- Lee, M. A. McHugh, J. Polym. Sci. 30 (1992) 1366

[41] H. Latz, Diplomarbeit, Göttingen (1999)

[42] H. Dietzsch, Dissertation, Göttingen (1999) 


\section{Ethen-(Meth)Acrylat-Copolymerisations- parameter}

\subsection{Grundlagen der Copolymerisationskinetik}

In diesem Abschnitt werden die für diese Arbeit wesentlichen Aspekte der Copolymerisationskinetik erläutert. Die radikalische Copolymerisation ist seit vielen Jahren Inhalt der Standardlehrbücher der Makromolekularen Chemie. Es wird daher darauf verzichtet, die Grundlagen der Copolymerisationskinetik und die Entwicklung der verschiedenen Modelle im Detail darzustellen. Hierzu wird auf die Literatur verwiesen, z.B. [1,2,3].

Die Reaktionschritte, mit denen die Copolymerisationskinetik beschrieben wird, lassen sich formal in zwei Typen gliedern:

(a) Reaktionsschritte, die mit einer Änderung der Monomerkonzentration und/oder der Radikalkonzentration verbunden sind. Diese Reaktionsschritte bestimmen den Umsatz der einzelnen Monomere und die Copolymerzusammensetzung.

(b) Reaktionsschritte, welche die Polymerstruktur/Molekulargewichtsverteilung verändern, ohne dass die Radikalkonzentration beeinflusst wird.

Reaktionsschritte vom Typus (b) beinhalten im wesentlichen Transferreaktionen. Hierbei wird die Radikalfunktion auf ein Makromolekül oder eine Monomereinheit übertragen. Die Behandlung der Copolymerisationskinetik beschränkt sich im folgenden auf Reaktionsschritte des Typus $a$ ).

Die Komplexität der mathematischen Beschreibung der Polymerisationskinetik reduziert sich erheblich, wenn folgende Grundannahmen für eine ideale Polymerisationskinetik getroffen werden können [3]:

- Die Polymerisation verläuft in homogener Phase.

- Alle Reaktionen verlaufen irreversibel.

- Die Monomere werden ausschließlich in der Wachstumsreaktion verbraucht und reagieren nur nach einem Mechanismus.

- Die Reaktivität der Makroradikale ist unabhängig von der Kettenlänge und der Polymermikrostruktur.

- Die Makroradikale besitzen große Kettenlängen. 
Die Reaktionsschritte des Typus (a) beinhalten folgende Vorgänge:

\section{Initiierung:}

Die radikalische Polymerisation wird durch Radikale initiiert, welche üblicherweise durch thermischen Zerfall eines Initiators oder eines Monomeren erzeugt werden. Im folgenden Reaktionsschema bezeichnet $k_{\mathrm{d}}$ die Zerfallskonstante:

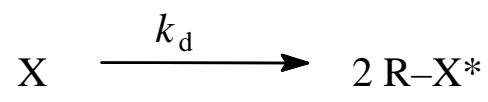

$\mathrm{X}$ steht für eine kleine Spezies (Initiator oder Monomer), R-X* für das hieraus gebildete Radikal. In dieser Arbeit wurde darauf abgezielt, die Copolymersynthesen bei möglichst geringen Umsätzen auszuführen, um Copolymere mit schmaler Molekulargewichtsverteilung und hoher chemischer Einheitlichkeit (vgl. Abschnitt 3.1.3) zu erhalten. Überwiegend wurde für die Synthesen die bisher bei allen Ethen-Copolymerisationen ab einer Temperatur von ca. $160^{\circ} \mathrm{C}$ beobachtete Selbstinitiierung ${ }^{1}$ der Monomermischungen genutzt. Aus diesem Grund wird der Aspekt Initiatorzerfall / Initiierung in dieser Arbeit nicht näher behandelt. Detaillierte Informationen bezüglich der Initiierungskinetik in der Hochdruck-Ethen-(Co)Polymerisation mit und ohne Anwendung von Initiatoren finden sich in [4, 5, 31].

\section{Kettenwachstum (Propagation)}

Im Kettenwachstumsschritt (Propagation) lagern die radikalischen Molekülenden (R) mit dem Wachstumsgeschwindigkeitskoeffizienten $k_{\mathrm{p}, \mathrm{m}}$ weitere Monomere an.

$$
\mathrm{R}-\mathrm{X}^{*}+\mathrm{M} \stackrel{k_{\mathrm{p}, \mathrm{M}}}{\longrightarrow} \mathrm{R}-\mathrm{X}-\mathrm{M}^{*}
$$

\section{Kettenabbruch (Terminierung)}

Der Abbruch des Kettenwachstums erfolgt entweder durch Kombination zweier Makroradikale $R_{r}$ und $R_{s}$ zu einem ( $n=1$ ) oder durch Disproportionierung zu zwei Makromolekülen $(n=2)$. Vereinfachend lassen sich diese Reaktionen durch einen Brutto-Geschwindigkeitskoeffizienten $k_{\mathrm{t}}$ beschreiben:

\footnotetext{
${ }^{1}$ Der Mechanismus der Selbstinitiierung bei Ethen-(Co)Polymerisationen ist bisher noch ungeklärt. Es kann nicht entschieden werden, ob es der Zerfall von Monomer oder ob es Verunreinigungen im (Meth)Acrylatmonomer sind, welche die Selbstinitiierung bewirken.
} 


$$
\mathrm{R}_{\mathrm{r}}+\mathrm{R}_{\mathrm{s}} \stackrel{k_{\mathrm{t}}}{\longrightarrow} \mathrm{nP}
$$

\subsection{Definition von Copolymerisationsparametern}

Der Copolymerisationsparameter $r_{\mathrm{i}}$ ist definiert als Verhältnis der Geschwindigkeitskoeffizienten von Homo- zu Kreuzwachstum.

$$
r_{\mathrm{i}}=k_{\mathrm{pi} 1} / k_{\mathrm{pi} 2}
$$

In Abhängigkeit von dem zur Beschreibung der Wachstumskinetik der Copolymerisation zweier Monomere $\mathrm{M}_{1}$ und $\mathrm{M}_{2}$ verwendeten Modell lassen sich unterschiedliche Copolymerisationsparameter definieren. Im einfachsten Fall, dem Terminal-Modell [6,7], werden die Makroradikale nur anhand ihrer die Radikalfunktion tragenden Einheit unterschieden, d.h. es wird davon ausgegangen, dass nur diese terminale Einheit die Reaktivität des wachsenden Radikals bestimmt. Das Modell unterscheidet somit folgende vier Wachstumsschritte und es ergeben sich hierdurch die beiden Copolymerisationsparameter $r_{1}$ und $r_{2}$, (Gl. 4.2 und 4.3).

$$
\begin{aligned}
& \mathrm{R}-\mathrm{M}_{1}+\mathrm{M}_{1} \stackrel{k_{\mathrm{p}, 11}}{\longrightarrow} \mathrm{R}-\mathrm{M}_{1} \mathrm{M}_{1} \\
& \mathrm{R}-\mathrm{M}_{1}+\mathrm{M}_{2} \stackrel{k_{\mathrm{p}, 12}}{\longrightarrow} \mathrm{R}-\mathrm{M}_{1} \mathrm{M}_{2} \\
& \mathrm{R}-\mathrm{M}_{2}+\mathrm{M}_{1} \stackrel{k_{\mathrm{p}, 21}}{\longrightarrow} \mathrm{R}-\mathrm{M}_{2} \mathrm{M}_{1} \\
& \mathrm{R}-\mathrm{M}_{2}+\mathrm{M}_{2} \stackrel{k_{\mathrm{p}, 22}}{\longrightarrow} \mathrm{R}-\mathrm{M}_{2} \mathrm{M}_{2}
\end{aligned}
$$

$$
r_{1}=k_{\mathrm{p}, 11} / k_{\mathrm{p}, 12}
$$

$$
r_{2}=k_{\mathrm{p}, 22} / k_{\mathrm{p}, 21}
$$

Im Unterschied zum Terminal-Modell berücksichtigt das erstmalig von Merz et al. [8] vorgeschlagene PEU-Modell (Penultimate-Modell) nicht nur den Einfluss der terminalen Monomereinheit, sondern darüber hinaus auch denjenigen der vorletzten Monomereinheit (penultimate unit) auf die Geschwindigkeitskoeffizienten des Kettenwachstums. Für eine binäre Copolymerisation ergeben sich hieraus acht Wachstumsreaktionen und vier Copoly- 
merisationsparameter (vgl. Gl. 4.4 - 4.7). Die Geschwindigkeitskoeffizienten $k_{\mathrm{p}, \mathrm{ijk}}$ beziehen sich dabei auf die Reaktion zwischen einem Monomer der Sorte k und einem Makroradikal mit i als vorletzter und $\mathrm{j}$ als endständiger Monomereinheit.

$$
\begin{aligned}
& r_{11}=\frac{k_{\mathrm{p}, 111}}{k_{\mathrm{p}, 112}} \\
& r_{22}=\frac{k_{\mathrm{p}, 222}}{k_{\mathrm{p}, 221}}
\end{aligned}
$$

$$
\begin{gathered}
r_{21}=\frac{k_{\mathrm{p}, 211}}{k_{\mathrm{p}, 212}} \\
r_{12}=\frac{k_{\mathrm{p}, 122}}{k_{\mathrm{p}, 121}}
\end{gathered}
$$

$r_{\mathrm{ij}}$ repräsentiert hier einen „Penultimate“-Copolymerisationsparameter. Die beiden weiteren Parameter des Penultimate-Modells, die Radikalreaktivitäten $s_{1}$ und $s_{2}$, charakterisieren den Effekt der vorletzten Monomereinheit j bei der Homowachstumsreaktion (Gl. 4.8 und 4.9).

$$
s_{1}=\frac{k_{\mathrm{p}, 211}}{k_{\mathrm{p}, 111}}
$$

$$
s_{2}=\frac{k_{\mathrm{p}, 122}}{k_{\mathrm{p}, 222}}
$$

Indem mittlere Copolymerisationsparameter $\bar{r}_{\mathrm{i}}$ und mittlere Kettenwachstumsgeschwindigkeitskoeffizenten $\bar{k}_{\mathrm{p}}$ eingeführt werden, können die Ergebnisse von Terminal- und Penultimate-Modell miteinander verknüpft werden. Für das Terminal-Modell entspricht der mittlere Copolymerisationsparameter $\bar{r}_{\mathrm{i}}$ dem mit Gleichung 4.1 allgemein definierten Copolymerisationsparameter $r_{\mathrm{i}}$. Den Zusammenhang zwischen den Copolymerisationsparametern aus Terminal- und Penultimate-Modell beschreiben die Gleichungen 4.10 bis 4.13:

$$
\begin{aligned}
& \bar{r}_{1}=r_{21} \cdot \frac{r_{11} \cdot f_{1}+f_{2}}{r_{21} \cdot f_{1}+f_{2}} \\
& \bar{r}_{2}=r_{12} \cdot \frac{r_{22} \cdot f_{2}+f_{1}}{r_{12} \cdot f_{2}+f_{1}}
\end{aligned}
$$




$$
\begin{gathered}
\bar{k}_{\mathrm{p}, 11}=k_{\mathrm{p}, 111} \cdot \frac{r_{11} \cdot f_{1}+f_{2}}{r_{11} \cdot f_{1}+\frac{f_{2}}{s_{1}}} \\
\bar{k}_{\mathrm{p}, 22}=k_{\mathrm{p}, 222} \cdot \frac{r_{22} \cdot f_{2}+f_{1}}{r_{22} \cdot f_{2}+\frac{f_{1}}{s_{2}}}
\end{gathered}
$$

$f_{\mathrm{i}}$ bezeichnet das Stoffmengenverhältnis von Monomer i bezogen auf die Gesamtstoffmenge der Monomermischung. Das vollständige PUE-Modell mit acht Wachstumsschritten und sechs Parametern $\left(r_{11}, r_{12}, r_{21}, r_{22}, s_{1}\right.$ und $\left.s_{2}\right)$ wird als Explizites-Penultimate- $\underline{\text { Unit-Effect }}$ Modell (EPUE-Modell) bezeichnet [9, 10].

Das Terminal-Modell ist das bislang zur Beschreibung von Copolymerisationen am häufigsten angewandte Modell. Dies resultiert aus der Einfachheit des Ansatzes und daraus, dass hiermit überwiegend gute Ergebnisse erhalten werden, wenn es allein darum geht die Abhängigkeit der Copolymerzusammensetzung von der Zusammensetzung der Monomermischung zu beschreiben. Unterschiede in der Eignung von Terminal und EPUE-Modell lassen sich daher nur in Ausnahmefällen allein auf der Basis von Daten für Comonomer- und Copolymerzusammensetzung feststellen.

Insbesondere durch die Entwicklung von Techniken zur exakten Bestimmung der den Monomerumsatz bestimmenden Kettenwachstumsgeschwindigkeit [11] hat sich gezeigt, dass bei fast allen Copolymersystemen Penultimate-Effekte wirksam sind [12]. Erkennbar wird dies daran, dass in der Regel mit dem Terminal zwar die Zusammensetzung und getrennt davon auch der Monomerumsatz gut beschrieben werden kann, dies jedoch nicht gleichzeitig mit einem Parametersatz möglich ist. Die Ursache dafür, dass die Copolymerzusammensetzung trotz des Einflusses von Penultimate-Einheiten gut beschrieben wird, liegt darin, dass im Terminal-Modell die Copolymerzusammmensetzung eine Funktion der Verhältnisse von Geschwindigkeitskoeffizienten ist [13]. Gerade bei dieser Verhältnisbildung der Koeffizienten des Homo- zum Kreuzwachstums von Radikalen mit gleicher terminaler und PenultimateEinheit sollte der hauptsächliche Einfluss der Penultimate-Wirkung verloren gehen. Der Kettenwachstumsgeschwindigkeitskoeffizient in der Copolymerisation $k_{\mathrm{p}, \text { copo }}$ ist hingegen zusätzlich eine Funktion von zwei Homowachstumsgeschwindigkeitskoeffizienten, deren Beeinflussung durch Penultimate-Einheiten nicht durch Verhältnisbildung kompensiert wird. 
Es sind bisher aus der Literatur nur wenige Copolymersysteme bekannt, für welche die Copolymerzusammensetzung und die Wachstumsgeschindigkeitskoeffizienten nicht mit dem Impliziten-Penultimate-Unit-Modell (s.u.), sondern erst durch Anwenden des EPUE-Modell $\mathrm{zu}$ beschreiben war. Überwiegend erweist sich ein reduziertes Penultimate-Modell, das Implizite-Penultimate-Unit-Modell (IPUE) [14] als ausreichend, um die beiden Größen zu beschreiben $[13,15,16]$.

Die Vereinfachung des IPUE-Modells besteht darin, dass aus zuvor genannten Gründen angenommen wird, dass die penultimate Einheiten keinen Einfluss auf die Quotienten aus Homo- zu Kreuzwachstums-Geschwindigkeitskoeffizient haben. Somit reduzieren sich die sechs Parameter des EPUE-Modells auf vier $\left(r_{1}, r_{2}, s_{1}, s_{2}\right)$, da gilt: $r_{22}=r_{12}=\bar{r}_{1}$ und $r_{11}=r_{21}=r_{1}$ sowie: $r_{22}=r_{21}=r_{2}$. Der (nicht durch Verhältnisbildung kompensierte) Effekt der Penultimate-Einheit auf den Copolymerisations-Wachstumsgeschwindigkeitskoeffizienten $k_{\mathrm{p} \text {, copo }}$ findet beim IPUE-Modell im Unterschied zum Terminal-Modell Berücksichtigung in Form der Radikalreaktivitäten $s_{1}$ und $s_{2}$.

Um zu entscheiden, welches der vorgestellten Modelle für ein bestimmtes Copolymersystem gültig ist, können zum einen Messungen der Wachstumsgeschwindigkeitskoeffizienten z.B. mit der PLP-SEC-Methode [17] vorgenommen werden. Diese auf eine Vielzahl von Systemen anwendbare Methode versagt aber, wenn bei höheren Temperaturen $k_{\mathrm{p}}$-Werte von reaktiven Monomeren bestimmt werden sollen. Die Kontrolle der Molekulargewichtsverteilung durch Laserpulsbestrahlung geht unter diesen Bedingungen durch zu hohe Geschwindigkeit der Kettenwachstumsreaktion als auch der Terminierungsreaktion verloren [18]. Für die im Rahmen dieser Arbeit untersuchten Systeme müssen, um die Synthese in homogener Phase ausführen zu können, Reaktionstemperaturen von $170^{\circ} \mathrm{C}$ und höher angewendet werden. Die PLP-SEC Methode ist für diese Bedingungen ungeeignet.

Eine weitere Möglichkeit um Hinweise zu erhalten, welches Modell gültig ist, besteht darin die Copolymerisationsparameter als Funktion der Zusammensetzung der Monomermischung, $f_{\mathrm{A}} \mathrm{zu}$ bestimmen. Wenn anhand experimenteller Daten gezeigt werden kann, dass die Copolymerisationsparameter $\bar{r}_{1}$ unabhängig von der gewählten Zusammensetzung der Monomermischung $f_{\mathrm{A}}$ sind, so gilt das Terminal- und/oder das IPUE-Modell $\left(r_{22}=r_{12}=\bar{r}_{1}\right)$. Wenn zusätzlich belegt wird, dass $\bar{k}_{\mathrm{p}, 22}$ und $\bar{k}_{\mathrm{p}, 21}$ von $f_{\mathrm{A}}$ abhängen, so gilt das Terminal-Modell als widerlegt und das IPUE-Modell als belegt. 
Für den Fall, dass sowohl die Copolymerisationsparameter als auch $\bar{k}_{\mathrm{p}, 22}$ und $\bar{k}_{\mathrm{p}, 21}$ Funktion der Zusammensetzung der Monomermischung $f_{\mathrm{A}}$ sind, gilt das Explizite-Penultimate-Modell $\left(r_{22} \neq r_{12} \neq \bar{r}_{1}\right)$.

Eine Methode um Copolymerisationsparameter in Abhängigkeit von der Zusammensetzung der Monomermischung $f_{\mathrm{A}}$ zu ermitteln, besteht darin die Parameter anhand der Häufigkeitsverteilung der Comonomersequenzen von Copolymermaterial zu bestimmen. Die Sequenzlängenverteilung entspricht vereinfachend ausgedrückt einem „kinetischen Fingerabdruck“, da die Häufigkeit von spezifischen Comonomersequenzen innerhalb des Copolymeren proportional zur Anlagerungswahrscheinlichkeit der Monomerbausteine ist [19]. Für die Analyse der Sequenzlängenverteilung wird üblicherweise die quantitative ${ }^{13} \mathrm{C}-\mathrm{NMR}-$ Spektroskopie eingesetzt. Während diese Methode erfolgreich für Copolymere von Ethen und Methylacrylat angewendet werden konnte [20] hat sich für Ethen-Butylacrylat-Copolymere gezeigt, dass die Sicherstellung der quantitativen Auswertbarkeit der Spektren problematisch ist [34]. Dies beruht vermutlich auf einer verzögerten Relaxation oder auch darauf, dass die Struktur von Ethen-Copolymeren mit längerkettigen Acrylaten komplexer ist, da in diesem Fall Übertragungsreaktionen auch auf die Esterseitenkette möglich sind. Aufgrund der erläuterten Schwierigkeiten wurde in dieser Arbeit auf eine ${ }^{13} \mathrm{C}$-Analyse verzichtet.

Eine weitere Methode um Copolymerisationsparameter in Abhängigkeit von $f_{\mathrm{A}} \mathrm{zu}$ bestimmen, nutzt die "First-Order-Markov-Chain"-Berechnung [19]. Mit diesem aus der Statistik stammenden Rechenmodell können wie bei der zuvor beschriebenen Methode im Prinzip Copolymerisationsparameter für jedes einzelne $F_{\mathrm{A}}-f_{\mathrm{A}}$-Wertepaar erhalten werden. v. Boxtel [20] hat diese Methode für das System E-MA, welches mit denen in dieser Arbeit behandelten Copolymersystemen eng verwandt ist, ausführlich untersucht. Hierbei zeigte sich, dass die erzielbare Genauigkeit der für einzelne $f_{\mathrm{A}}-F_{\mathrm{A}}$-Wertepaare berechneten Copolymerisationsparameter vergleichsweise gering ist und damit keine Beurteilung über die Gültigkeit von Copolymerisationsmodellen erlaubt.

Sehr häufig wird zur Bestimmung von Copolymerisationsparametern binärer Systeme die sogenannte Lewis-Mayo-Gleichung (Gl. 4.14) auf experimentelle $f_{\mathrm{A}}-F_{\mathrm{A}}$-Wertepaare angewandt. Gleichung Gl. 4.14 gilt unter Annahme idealer Polymerisationskinetik für das Terminal-Modell und das Implizite-Penultimate-Modell. Diese Gleichung beschreibt die Abhängigkeit der Copolymerzusammensetzung, charakterisiert durch den Stoffmengenanteil von eingebauter Monomereinheit A, von der Zusammensetzung der Reaktionsmischung, charakterisiert durch den Stoffmengenanteil von Monomer A. Der Index E steht für die zweite Monomereinheit. 


$$
F_{\mathrm{A}}=\frac{r_{\mathrm{A}} \cdot f_{\mathrm{A}}^{2}+f_{\mathrm{A}} \cdot f_{\mathrm{E}}}{r_{\mathrm{A}} \cdot f_{\mathrm{A}}^{2}+2 \cdot f_{\mathrm{A}} \cdot f_{\mathrm{E}}+r_{\mathrm{E}} \cdot f_{\mathrm{E}}^{2}}
$$

Die Voraussetzung idealer Polymerisationskinetik (s.o.) bedeutet unter anderem, dass die Synthese in homogener Phase und bei lediglich differentiellem Monomerumsatz oder unter stationären Bedingungen abläuft, und dass keine Depropagation und / oder andere Nebenreaktionen auftreten. Es wird allgemein angenommen, dass bei Acrylat-Polymerisationen im technisch angewandten Temperaturintervall von ca. $80-280^{\circ} \mathrm{C}$ keine Depropagation auftritt, bzw. dass der Einfluss der Depropagation unerheblich ist. Bei Polymerisationen von Methacrylaten bei hohen Temperaturen ist die Depropagation jedoch ein gut bekanntes Phänomen [21, 22]. Für die Copolymerisation von Ethen mit Methylmethacrylat haben Buback et al. [23] Hinweise auf den Einfluss von Depropagation in Form nichtlinearen Verhaltens von $\ln r_{\mathrm{A}}$ gegen $T^{-1}$ gefunden. Dies wurde jedoch erst bei Temperaturen von $250^{\circ} \mathrm{C}$ und höher beobachtet. Im Rahmen dieser Arbeit wurden Copolymerisationen bis maximal $240^{\circ} \mathrm{C}$ durchgeführt. Für die weitere Auswertung wird daher davon ausgegangen, dass die Depropagation keinen Einfluss hat und die Voraussetzung für die Anwendung der Lewis-Mayo-Gleichung erfüllt ist.

Die Lewis-Mayo-Gleichung hat gegenüber den vorgenannten Methoden zur Bestimmung von Copolymerisationsparametern den Nachteil, dass sie nicht für einzelne $F_{\mathrm{A}}-f_{\mathrm{A}}-$ Wertepaare Copolymerisationsparameter liefert sondern immer nur auf eine Reihe von mindestens vier Wertepaaren angewendet werden kann. Zur Beantwortung der Frage, inwieweit die Copolymerisationsparameter eine Funktion der Comonomerzusammensetzung sind, können jedoch verschieden große Bereiche einer Serie von $F_{\mathrm{A}}-f_{\mathrm{A}}$-Wertepaaren ausgewertet werden. Hierbei werden sozusagen integrale Copolymerisationsparameter für Teilbereiche der Comonomerzusammensetzung erhalten. In Abbildung 4.1 wird beispielhaft das Resultat eines solchen Vorgehens gezeigt. Dargestellt sind Auftragungen der Copolymerzusammensetzungen gegen die Zusammensetzungen der Comonomermischung für das Copolymersystem Ethen-Propylacrylat bei einer Temperatur von $170^{\circ} \mathrm{C}$. Durch Anpassen von Gleichung 4.14 an die experimentellen Datenpaare wurden für die folgenden Bereiche mittlere Copolymerisationsparameter bestimmt: a) $0<f_{\mathrm{A}}<0.02$, b) $0<f_{\mathrm{A}}<0.0375$, c) $0<f_{\mathrm{A}}<0.064$. 
Die Ergebnisse der Anpassungen sind in den Auftragungen als Linien eingezeichnet. Der Fitbereich ist als durchgezogene Linie dargestellt, der vorausberechnete Verlauf der Copolymerzusammensetzung ist gestrichelt gezeichnet. Die erhaltenen Copolymerisationsparameter sind in den Grafiken angegeben.

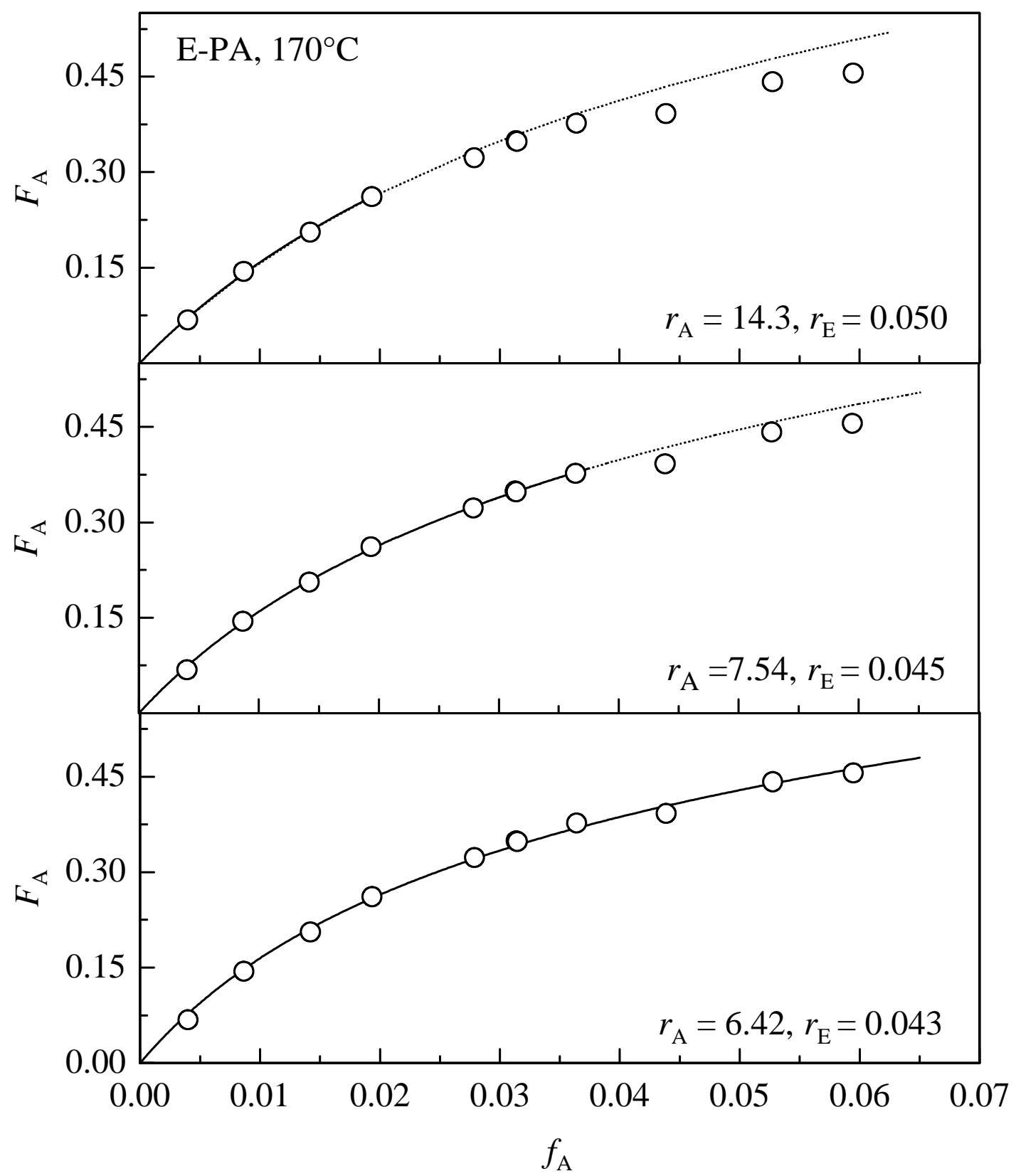

Abbildung 4.1: Lewis-Mayo-Auftragungen für Copolymerisationen von Ethen mit Propylacrylat bei 2000 bar und $170^{\circ} \mathrm{C}$ im CSTR. Die Daten wurden zur Ermittlung der Copolymerisationsparameter mit Gl. 4.14 in unterschiedlichen weiten Bereichen von $f_{\mathrm{A}}$ angepasst (durchgezogene Linie). Die gestrichelte Linie gibt den unter Anwendung der jeweils rechts unten angegebenen Copolymerisationsparametern vorausberechneten Verlauf der Copolymerzusammensetzung an. 
Es zeigt sich, dass die beiden, nur für einen Teil des untersuchten Zusammensetzungsbereich erhaltenen Anpassungen a) und b) die experimentellen Ergebnisse bei höheren Acrylatgehalten in der Comonomermischung jeweils überschätzen. Die Datenpunkte bei geringen $f_{\mathrm{A}^{-}}$ Werten werden in allen Fällen gut wiedergegeben, allerdings bei sehr unterschiedlichen Zahlenwerten für $r_{\mathrm{A}}$. Für das dargestellte Beispiel nimmt der Copolymerisationsparameter $r_{\mathrm{A}}$ mit zunehmender Breite des für den Fit genutzten Bereiches von 14.3 auf 6.4 ab.

Auch für die anderen in dieser Arbeit untersuchten Copolymersysteme (E-EA, E-MMA, EEMA, E-BMA und E-BzMA) und weiterhin für die Literaturdaten zu E-MA [20] wird analog der zuvor erläuterten Auswertung von Teilbereichen der $f_{\mathrm{A}}-F_{\mathrm{A}}$-Daten gefunden, dass die Copolymerisationsparameter zumindest geringfügig vom Umfang des für den Fit verwendeten $f_{\mathrm{A}}$-Bereichs abhängen.

Diese Beobachtung kann allerdings nicht als zweifelsfreier Beleg für das Auftreten von Penultimate-Effekten interpretiert werden, da zum einen die mit der beschriebenen Methode bestimmten Copolymerisationsparameter aufgrund der z.T. geringen Datenmenge und des begrenzten untersuchten Zusammensetzungsbereich der Monomermischung eine vergleichsweise große Unsicherheit aufweisen (vgl. Abschnitt 4.4). Zum anderen zeigen die Ergebnisse von v. Boxtel [20] dass die Auswertung der Sequenzlängenverteilung für das System E-MA in einem weiten Zusammensetzungsbereich nahezu identische Copolymerisationsparameter ergibt obwohl für das gleiche experimentelle Material mit der Lewis-Mayo-Methode deutlich von der Zusammensetzung abhängige Parameter berechnet werden. Es ist daher anzunehmen, dass das Auffinden einer Abhängigkeit der Copolymerisationsparameter von der Copolymerzusammensetzung mit der Lewis-Mayo-Methode auf anderen Ursachen als auf dem Auftreten von expliziten Penultimate-Effekten beruht.

Prinzipiell mögliche Ursachen sind Depropagationsphänomene und andere von der idealen Polymerisationskinetik abweichende Vorgänge. Da auch bei der niedrigsten angewandten Temperatur $\left(170^{\circ} \mathrm{C}\right)$ die beschriebene Abhängigkeit gefunden wird ist die Depropagation unwahrscheinlich. Es kann vermutet werden, dass die bei Anwendung eines nur schmalen Bereichs der Comonomerzusammensetzung besonders hohe Sensitivität der Auswertung nach Lewis-Mayo auf Ungenauigkeiten in der Copolymerzusammensetzung die Ursache darstellt. Hierbei ist auch zu berücksichtigen, dass der Fehler der ${ }^{1} \mathrm{H}-\mathrm{NMR}$ spektroskopisch bestimmten Copolymerzusammensetzung mit dem Meth)Acrylatgehalt im Copolymer zunimmt (vgl. Abschnitt 4.4). Bei der Bestimmung der Zusammensetzung mittels Elementaranalyse nimmt die Unsicherheit in dergleichen Richtung ebenfalls $\mathrm{zu}$ [31]. Es kann daher nicht ausgeschlossen werden, dass die bei Auswertung mit der Lewis-Mayo-Methode sich an- 
deutende Abhängigkeit der Copolymerisationsparameter von der Copolymerzusammensetzung lediglich auf Messunsicherheiten beruhen. In diesem Zusammenhang soll darauf hingewiesen werden, dass die im Rahmen dieser Arbeit angewandte ${ }^{1} \mathrm{H}-\mathrm{NMR}$-Analyse die verlässlichste Methode zur Bestimmung der Copolymerzusammensetzung ist. Dies liegt daran, dass im Unterschied zur Elementaranalyse Verfälschungen durch Restmonomergehalt erkannt werden können und auch keine Störungen durch Relaxationsprobleme [34] wie bei der ${ }^{13}$ C-NMR-Merthode auftreten.

Für die im Rahmen dieser Arbeit untersuchten Systeme des Typs Ethen-(Meth)Acrylat wird für die weitere Auswertung angenommen, dass das Terminal / IPUE-Modell gültig ist. Das explizite Penultimate-Modell ist, wie zuvor erläutert, anhand des experimentellen Datenmaterials nicht belegbar und für das verwandte Copolymersystem E-MA ebenfalls nicht gefunden worden. Als Konsequenz der beobachteten Abhängigkeit der Copolymerisationsparameter vom Umfang des zur Auswertung verwendeten Bereichs der Comonomerzusammensetzung erscheint es sinnvoll, die Daten aller Copolymersysteme innerhalb eines gleichen (beschränkten) $f_{\mathrm{A}}$-Bereichs auszuwerten. Für die im Rahmen dieser Arbeit untersuchten Systeme E-EA und E-PA sowie für die Literaturdaten für E-BA wird hierzu der Bereich von $f_{\mathrm{A}}=0-0.038$ gewählt. Der Umfang dieses Bereichs stellt einen Kompromiss dar. Bei einem noch schmaleren Bereich sind für einige Systeme für die Auswertung nicht ausreichend Messpunkte vorhanden.

Es sei angemerkt, dass die Beschränkung des für die Auswertung verwendeten Comonomerzusammensetzungsbereichs auch vor dem Hintergrund des technischen Interesses gerechtfertigt ist, da der hiermit berücksichtigte Bereich der Copolymerzusammensetzung bis ca. 35 mol-\% den Zusammensetzungsbereich industriell produzierter Ethen-Acrylat-Copolymere umfasst. Für das E-MA-System werden beim Vergleich mit Literaturdaten (s. Abschnitt 4.6) die aus der Auswertung der Sequenzlängenverteilung bestimmten Copolymerisationsparameter [20] herangezogen.

Für die im Rahmen dieser Arbeit untersuchten Ethen-Methacrylat-Systeme wird nur eine vernachlässigbar geringe Abhängigkeit der Copolymerisationsparameter vom Auswertungsbereich gefunden. Diese Beobachtung stimmt mit den Ergebnissen von Buback et al. [23] für E-MMA überein. Die Autoren haben jedoch für Temperaturen von $250^{\circ} \mathrm{C}$ und $290^{\circ} \mathrm{C}$ Abweichungen festgestellt, wenn zur Auswertung auch der Bereich der Comonomerzusammensetzung oberhalb von 7 mol-\% herangezogen wird. Die Autoren interpretieren dies als Hinweis auf den Einfluß von Depropagationsreaktionen unter diesen extremen Bedingungen. In dieser Arbeit wurden Copolymerisationen nur bis maximal $240^{\circ} \mathrm{C}$ und 
zudem nur bis zu einem Methacrylatgehalt in der Comonomermischung von 6.5 mol-\% durchgeführt, so dass es gerechtfertigt scheint Depropagationsphänomene zu vernachlässigen. Ergänzend soll erwähnt werden, dass neben den zuvor erläuterten Modellen, welche am häufigsten Anwendung finden, eine Reihe weiterer Modelle in der Literatur beschrieben sind, die ebenfalls jeweils erfolgreich zur Beschreibung von Copolymerisationen eingesetzt wurden [24, 25, 26]. Die Schwierigkeit, über die Eignung hinaus die Gültigkeit eines bestimmten Modells nachzuweisen, ist in [27, 28] dokumentiert.

\subsection{Bestimmung von Ethen-(Meth)Acrylat-Copolymerisations- parametern}

Im folgenden Abschnitt wird das Auswerteverfahren beschrieben, mit dem die Copolymerzusammensetzung, der Umsatz und die Zusammensetzung der Monomermischung bestimmt wurden.

\subsubsection{Bestimmung der Copolymerzusammensetzung}

Der Stoffmengenanteil der synthetisierten Copolymere an (Meth)Acrylat $F_{\mathrm{A}}$ wird ${ }^{1} \mathrm{H}-\mathrm{NMR}$ spektroskopisch bestimmt. Die Methodik der (Meth)Acrylat-Gehaltsbestimmung ist in Abschnitt 3.2 detailliert beschrieben. Die Richtigkeit der erhaltenen Werte für $F_{\mathrm{A}}$ wurde anhand von Stichproben mittels Elementaranalyse geprüft. Zur weiteren Kontrolle wurden Korrelationen der $F_{\mathrm{A}}$-Werte mit Ergebnissen aus der IR-spektroskopischen Untersuchung der Copolymere erstellt (s. Abschnitt 3.2.4).

\subsubsection{Bestimmung der Zusammensetzung der Comonomermischung}

Der Stoffmengenanteil an (Meth)Acrylat in der Comonomermischung $f_{\mathrm{A}}$, welcher sich im stationären Zustand der Copolymersynthese in einem kontinuierlich betriebenen Rührkessel (CSTR) einstellt, lässt sich über die Massenbilanz der Komponenten bestimmen. Da die Monomere Ethen und (Meth)Acrylat bei der Reaktion in stark unterschiedlichem Maß in das gebildete Copolymer eingebaut werden, verarmt die Reaktionsmischung nach Einströmen in den Reaktor in Abhängigkeit vom Gesamtumsatz am jeweils reaktiverem Monomer (dem (Meth)Acrylat). Bei der Berechnung des (Meth)Acrylat-Stoffmengenanteils muss daher der Stoffmengenfluss $\dot{n}_{\mathrm{P}}$ sowie die Zusammensetzung $F_{\mathrm{A}}$ des gebildeten Copolymers berücksichtigt werden. 
Die Stoffmengenflüsse der Monomere Ethen $\dot{n}_{\mathrm{E}}$ und (Meth)Acrylat $\dot{n}_{\mathrm{A}}$ berechnen sich als Quotienten aus den jeweils vor dem Reaktor gemessenen Massenflüssen $\dot{m}_{\mathrm{E}}$ und $\dot{m}_{\mathrm{A}}$ und den entsprechenden Molmassen $M_{\mathrm{i}}\left(\dot{n}_{\mathrm{i}}=\dot{m}_{\mathrm{i}} / M_{\mathrm{i}}\right)$. Hierbei wird der Ethenfluss $\dot{m}_{\mathrm{E}}$ mit dem Massenflussregler (vgl. Abschnitt 3.1.2) eingestellt und geregelt. Die Zudosierungsgeschwindigkeit des (Meth)Acrylats $\dot{m}_{\mathrm{A}}$ wird direkt von der Anzeige der Dosierpumpensteuerung erhalten. Die eingestellten Flüsse können alternativ auch aus der Gewichtsabnahme im (Meth)Acrylat-Vorratgefäß bestimmt werden.

Der Stoffmengenfluss des Copolymeren $\dot{n}_{\mathrm{P}}$ berechnet sich aus aus dem Massenfluss $\dot{m}_{\mathrm{P}}$ des Copolymeren und der mittleren Molmasse einer Monomereinheit im Copolymer $\left(\bar{M}_{\mathrm{P}}=F_{\mathrm{A}} \cdot M_{\mathrm{A}}+\left(1-F_{\mathrm{A}}\right) \cdot M_{\mathrm{E}}\right)$. Der Copolymermassenfluss wird durch Auswaage des während eines definierten Zeitraums aufgefangenen Probenmaterials ermittelt. Für den Stoffmengenfluss des Copolymeren $\dot{n}_{\mathrm{P}}$ gilt:

$$
\dot{n}_{\mathrm{P}}=\frac{\dot{m}_{\mathrm{P}}}{\bar{M}_{\mathrm{P}}}=\frac{\dot{m}_{\mathrm{P}}}{F_{\mathrm{A}} \cdot M_{\mathrm{A}}+\left(1-F_{\mathrm{A}}\right) \cdot M_{\mathrm{E}}}
$$

Somit ergibt sich der im kontinuierlich betriebenen Rührkessel im stationären Zustand einstellende Stoffmengenanteil (Meth)Acrylat aus folgendem Ausdruck:

$$
f_{\mathrm{A}}=\frac{\dot{n}_{\mathrm{A}}-F_{\mathrm{A}} \cdot \dot{n}_{\mathrm{P}}}{\dot{n}_{\mathrm{A}}+\dot{n}_{\mathrm{E}}-\dot{n}_{\mathrm{P}}}=\frac{\frac{\dot{m}_{\mathrm{A}}}{M_{\mathrm{A}}}-\frac{\dot{m}_{\mathrm{P}} \cdot F_{\mathrm{A}}}{\bar{M}_{\mathrm{P}}}}{\frac{\dot{m}_{\mathrm{A}}}{M_{\mathrm{A}}}+\frac{\dot{m}_{\mathrm{E}}}{M_{\mathrm{E}}}-\frac{\dot{m}_{\mathrm{P}}}{\bar{M}_{\mathrm{P}}}}
$$




\subsection{Gewichtung der Daten - Berechnung der Fehlerstruktur}

Die Copolymerisationsparameter für die verschiedenen Ethen-(Meth)Acrylat-Systeme werden in dieser Arbeit durch Anpassen der differentiellen Lewis-Mayo-Gleichung (G1. 4.14) an die experimentellen $F_{\mathrm{A}}-f_{\mathrm{A}}$-Wertepaare mittels nichtlinearer Regression ermittelt. Hierzu werden die Parameter in einem weiten Wertebereich variiert und die zugehörigen Summen der

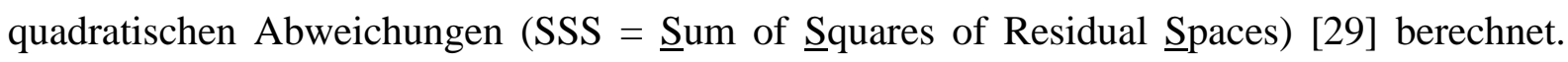
Das Minimum im derart ermittelten Summenraum gibt den optimalen Satz Copolymerisationsparameter an. Diese Vorgehensweise hat im Vergleich zu einer rein iterativen Anpassung der Lewis-Mayo-Gleichung den Vorteil, dass keine Artefakte durch Ermittlung von etwaigen lokalen Minima auftreten. Darüber hinaus erlaubt die Methode eine exaktere Anpassung der Lewis-Mayo-Gleichung an den experimentellen Datensatz, da sie die Möglichkeit einer Wichtung der Messpunkte vorsieht. Desweiteren können anhand der Ergebnisse die Konfi-

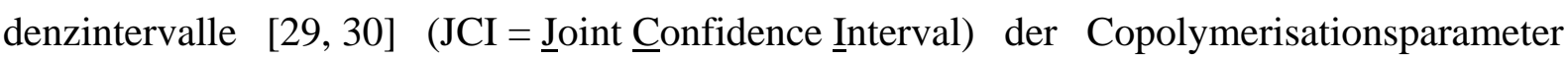
bestimmt werden.

Die Summe der quadratischen Abweichungen (SSS) berechnet sich bei $n$ Datenpunkten für die Copolymerisationsparamter $r_{1}$ und $r_{2}$ wie folgt:

$$
\operatorname{ss}\left(r_{1}, r_{2}\right)=\sum_{\mathrm{i}=1}^{\mathrm{i}=\mathrm{n}}\left\{w_{\mathrm{i}} \cdot\left[y_{\mathrm{i}}-f\left(x_{\mathrm{i}}, r_{1}, r_{2}\right)\right]^{2}\right\}
$$

Hierbei ist $w_{\mathrm{i}}$ der Gewichtungsfaktor für den Datenpunkt i. $f\left(x, r_{1}, r_{2}\right)$ beschreibt die Funktion, welche die unabhängige Variable $x_{\mathrm{i}}$ (hier $f_{\mathrm{A}}$ ) mit der abhängigen Variablen $y_{\mathrm{i}}$ (hier $F_{\mathrm{A}}$ ) verknüpft. Bei der Berechnung des SSS können die experimentellen Daten grundsätzlich nach folgenden drei Methoden gewichtet werden: 
a) Konstanter absoluter Fehler:

Wenn bekannt ist bzw. angenommen werden kann, dass die Fehler der Messwerte im gesamten Messbereich gleich groß und unabhängig vom Wert der Messgröße sind, beträgt der Gewichtungsfaktor $w_{\mathrm{i}}=1$. Dies bedeutet, dass alle experimentellen Datenpunkte gleich stark gewichtet werden.

\section{b) Konstanter relativer Fehler:}

Wenn bekannt ist bzw. angenommen werden kann, dass der Fehler proportional zum Wert der Messgröße ist, berechnet sich der Gewichtungsfaktor aus der abhängigen Variable wie folgt:

$$
w_{\mathrm{i}}=1 /\left[f\left(x_{\mathrm{i}}, r_{1}, r_{2}\right)\right]^{2}
$$

\section{c) Individueller Fehler:}

Für den Fall, dass der funktionelle Zusammenhang zwischen Fehler und Messgröße bekannt ist oder berechnet werden kann, kann die Anpassung unter Berücksichtigung des jeweiligen Fehler des einzelnen Datenpaares erfolgen. Hierbei berechnet sich der Gewichtungsfaktor aus der bekannten oder berechneten Varianz (bzw. Standardabweichung) des einzelnen Messwertes [31].

In Fällen, in denen keine Kenntnisse über die Fehlerstruktur vorhanden sind oder lediglich vereinfachende Annahmen gemacht werden können, muss auf Schema a) bzw. b) ausgewichen werden. Allgemein ist die Verwendung individueller Fehler (Methode $c$ ) bei der Wichtung zu bevorzugen, da sie die exakteste Anpassung an experimentelle Datenpunkte liefert. Informationen über die Ergebnisse der verschiedenen Gewichtungsschemata bei der Bestimmung von Copolymerisationsparametern für Ethen-Acrylat-Systeme finden sich in [31]. Im Rahmen dieser Arbeit sollen die individuellen Fehler der Messwerte zur Auswertung verwendet werden. Im folgenden Abschnitt wird die Unsicherheit der einzelnen Messgrößen untersucht und dargestellt, wie sich hieraus die individuellen Fehler der $F_{\mathrm{A}}-f_{\mathrm{A}}$-Wertepaare errechnen. 


\section{Bestimmung individueller Fehler}

Verwendet man Gleichung 4.17 zur Berechnung der SSS wird zunächst lediglich der Fehler der abhängigen Variable $\Delta F_{\mathrm{A}}$ nicht jedoch derjenige der unabhängigen Variable $\Delta f_{\mathrm{A}}$ berücksichtigt. Da der Fehler von $f_{\mathrm{A}}$, hervorgerufen durch fehlerbehaftete Monomerdosierflüsse und Fehler bei der Bestimmung des Umsatzes, nicht vernachlässigt werden kann, wird ähnlich der beim sogenannten EVM-Modell (Error in all Variables Model) angewandten Methode [32] der Fehler der unabhängigen Variable $f_{\mathrm{A}}$ in einen zusätzlichen Fehler der Variable $F_{\mathrm{A}}$ transponiert [31]. Der Gesamtfehler der Copolymerzusammensetzung $\Delta F_{\mathrm{A}}$ besteht damit aus dem Fehler der NMR-spektroskopisch bestimmten Zusammensetzung

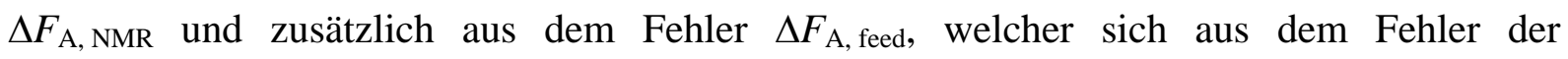
Zusammensetzung der Comonomermischung errechnet. Für den Gesamtfehler gilt folgender Ausdruck:

$$
\Delta F_{\mathrm{A}}=\sqrt{\left(\Delta F_{\mathrm{A}, \mathrm{NMR}}\right)^{2}+\left(\Delta F_{\mathrm{A}, \text { feed }}\right)^{2}}
$$

Im folgenden Abschnitt wird die Bestimmung der Fehler $\Delta F_{\mathrm{A}, \mathrm{NMR}}$ und $\Delta F_{\mathrm{A} \text {, feed }}$ erläutert.

\section{Bestimmung von $\Delta F_{A, N M R}$}

Der Fehler bei der Bestimmung des (Meth)Acrylatanteils im Copolymeren, $\left(\Delta F_{\mathrm{A}, \mathrm{NMR}}\right)$, resultiert im wesentlichen aus Ungenauigkeiten, welche bei der Auswertung der NMRSpektren durch die Phasierung, die Basislinienkorrektur und die Integration auftreten. Die NMR-Signale der verschiedenen Monomerbausteine in den Spektren sind auch bei geringen (Meth)Acrylatgehalten im Copolymer um ein Vielfaches größer als das Rauschen. Es kann daher davon ausgegangen werden, dass das Ausmaß der Ungenauigkeit der Integration unabhängig von der Copolymerzusammensetzung ist. Auf der Basis von Ergebnissen wiederholt ausgeführter Aufnahmen und Auswertungen einzelner Spektren wird die Genauigkeit der NMR-spektroskopisch bestimmten Copolymerzusammensetzung $F_{\mathrm{A} \text {, NMR }}$ auf \pm 1.3 mol- $\%$ abgeschätzt. 


\section{Berechnung von $\Delta F_{A, \text { feed }}$}

Der Anteil $\Delta F_{\mathrm{A} \text {, feed }}$ am Gesamtfehler von $F_{\mathrm{A}}$ resultiert aus dem Fehler bei der Bestimmung der Feedzusammensetzung $\Delta f_{\mathrm{A}}$. Zur Berechnung wird die partielle Ableitung der differentiellen Copolymerisationsgleichung 4.14 nach $f_{\mathrm{A}}$ gebildet.

$$
\Delta F_{\mathrm{A}, \text { feed }}=\left|\frac{\partial F_{\mathrm{A}}}{\partial f_{\mathrm{A}}} \cdot \Delta f_{\mathrm{A}}\right|
$$

mit

$$
\frac{\partial F_{\mathrm{A}}}{\partial f_{\mathrm{A}}}=\frac{f_{\mathrm{A}}{ }^{2} \cdot\left(r_{\mathrm{A}}-r_{\mathrm{E}}-2 \cdot r_{\mathrm{A}} \cdot r_{\mathrm{E}}\right)+f_{\mathrm{A}} \cdot\left(2 \cdot r_{\mathrm{A}} \cdot r_{\mathrm{E}}-2 \cdot r_{\mathrm{E}}\right)+r_{\mathrm{E}}}{\left[r_{\mathrm{A}} \cdot f_{\mathrm{A}}{ }^{2}+2 \cdot f_{\mathrm{A}}-2 \cdot f_{\mathrm{A}}{ }^{2}+r_{\mathrm{E}} \cdot\left(1-f_{\mathrm{A}}\right)^{2}\right]^{2}}
$$

Die Feedzusammensetzung $f_{\mathrm{A}}$ wird nach Gleichung 4.16 aus den Monomerflüssen und dem Fluss des ausgetragenen Copolymeren berechnet. Entsprechend setzt sich der Fehler von $f_{\mathrm{A}}$ aus den Fehlern der beiden Monomerflüsse und dem des Copolymerflusses zusammen. Der Fehler des Ethenmassenflusses beträgt nach Herstellerangabe des verwendeten Massenflussreglers $1 \%$ vom Skalenendwert $(2 \mathrm{~kg} / \mathrm{h}$ ). Für die im Rahmen dieser Arbeit ausgeführten Syntheseexperimente beläuft sich die Ungenauigkeit daher auf $\pm 20 \mathrm{~g} / \mathrm{h}$. Für eine Abschätzung des durch die Dosierpumpe verursachten Fehlers des (Meth)Acrylatflusses wurden bei verschiedenen Dosierraten die Dosierflüsse gravimetrisch kontrolliert. Dabei ergaben sich Abweichungen von maximal $\Delta \dot{V}_{\mathrm{A}}=3 \%$ zwischen dem gravimetrisch gemessenen und dem an der Pumpensteuerung eingestellten (Soll)Fluss. Dieser Wert wird in der weiteren Auswertung für die Ungenauigkeit des (Meth)Acrylat-Dosierstroms verwendet.

Für die Berechnung von $\Delta f_{\mathrm{A}}$ muss neben den Fehlern der beiden Monomerflüsse auch derjenige des Copolymerflusses (vgl. Gleichung 4.16) berücksichtigt werden. Es gilt:

$$
\Delta f_{\mathrm{A}}=\sqrt{\sum_{\mathrm{i}}\left(\frac{\partial f_{\mathrm{A}}}{\partial \dot{n}_{\mathrm{i}}} \cdot \Delta \dot{n}_{\mathrm{i}}\right)^{2}+\left(\frac{\partial f_{\mathrm{A}}}{\partial F_{\mathrm{A}}} \cdot \Delta F_{\mathrm{A}}\right)^{2}}
$$


Die zugehörigen partiellen Ableitungen aus Gleichung 4.22 berechnen sich nach:

$$
\begin{aligned}
& \frac{\partial f_{\mathrm{A}}}{\partial \dot{n}_{\mathrm{A}}}=\frac{\dot{n}_{\mathrm{E}}+\left(F_{\mathrm{A}}-1\right) \cdot \dot{n}_{\mathrm{P}}}{\left(\dot{n}_{\mathrm{A}}+\dot{n}_{\mathrm{E}}-\dot{n}_{\mathrm{P}}\right)^{2}} \\
& \frac{\partial f_{\mathrm{A}}}{\partial \dot{n}_{\mathrm{E}}}=\frac{\dot{n}_{\mathrm{A}}-F_{\mathrm{A}} \cdot \dot{n}_{\mathrm{P}}}{\left(\dot{n}_{\mathrm{A}}+\dot{n}_{\mathrm{E}}-\dot{n}_{\mathrm{P}}\right)^{2}} \\
& \frac{\partial f_{\mathrm{A}}}{\partial \dot{n}_{\mathrm{P}}}=\frac{2 \cdot F_{\mathrm{A}} \cdot \dot{n}_{\mathrm{P}}-\left(F_{\mathrm{A}}-1\right) \cdot \dot{n}_{\mathrm{A}}-F_{\mathrm{A}} \cdot \dot{n}_{\mathrm{E}}}{\left(\dot{n}_{\mathrm{A}}+\dot{n}_{\mathrm{E}}-\dot{n}_{\mathrm{P}}\right)^{2}} \\
& \frac{\partial f_{\mathrm{A}}}{\partial F_{\mathrm{A}}}=-\frac{\dot{n}_{\mathrm{P}}}{\left(\dot{n}_{\mathrm{A}}+\dot{n}_{\mathrm{E}}-\dot{n}_{\mathrm{P}}\right)^{2}}
\end{aligned}
$$

Es ist zu beachten, dass der Fehler des Stoffmengenflusses des Copolymeren auch den Fehler der gravimetrischen Umsatzbestimmung und zusätzlich den Fehler der Copolymerzusammensetzung beinhaltet.

$$
\Delta \dot{n}_{\mathrm{P}}=\sqrt{\left(\frac{\partial \dot{n}_{\mathrm{P}}}{\partial \dot{m}_{\mathrm{P}}} \cdot \Delta \dot{m}_{\mathrm{P}}\right)^{2}+\left(\frac{\partial \dot{n}_{\mathrm{P}}}{\partial F_{\mathrm{A}}} \cdot \Delta F_{\mathrm{A}}\right)^{2}}
$$

mit

$$
\begin{aligned}
\frac{\partial \dot{n}_{\mathrm{P}}}{\partial \dot{m}_{\mathrm{P}}} & =\frac{1}{F_{\mathrm{A}} \cdot M_{\mathrm{A}}+F_{\mathrm{E}} \cdot M_{\mathrm{E}}} \\
\frac{\partial \dot{n}_{\mathrm{P}}}{\partial F_{\mathrm{A}}} & =\frac{\dot{m}_{\mathrm{P}} \cdot\left(M_{\mathrm{A}}-M_{\mathrm{E}}\right)}{\left(F_{\mathrm{A}} \cdot M_{\mathrm{A}}+F_{\mathrm{E}} \cdot M_{\mathrm{E}}\right)^{2}}
\end{aligned}
$$

Die Unsicherheit bei der Bestimmung des Copolymerflusses durch Wägung des in einem definierten Zeitraum produzierten Copolymeren mit einer Laborwaage (Sartorius, BP 1500) wird $\mathrm{zu} \pm 0.2 \mathrm{~g}$ abgeschätzt. 


\section{Berechnung von $\Delta F_{\mathrm{A}}$}

Der Gesamtfehler der Copolymerzusammensetzung $\Delta F_{\mathrm{A}}$ wird nach Gleichung 4.19 aus den Einzelfehlern $\Delta F_{\mathrm{A} \text {, feed }}$ und $\Delta F_{\mathrm{A} \text {, NMR }}$ berechnet. In Abbildung 4.2 ist die Abhängigkeit der drei Fehler von der Copolymerzusammensetzung für das Beispiel einer Ethen-EthylacrylatCopolymerisation bei $170{ }^{\circ} \mathrm{C}$ und 2000 bar aufgetragen.

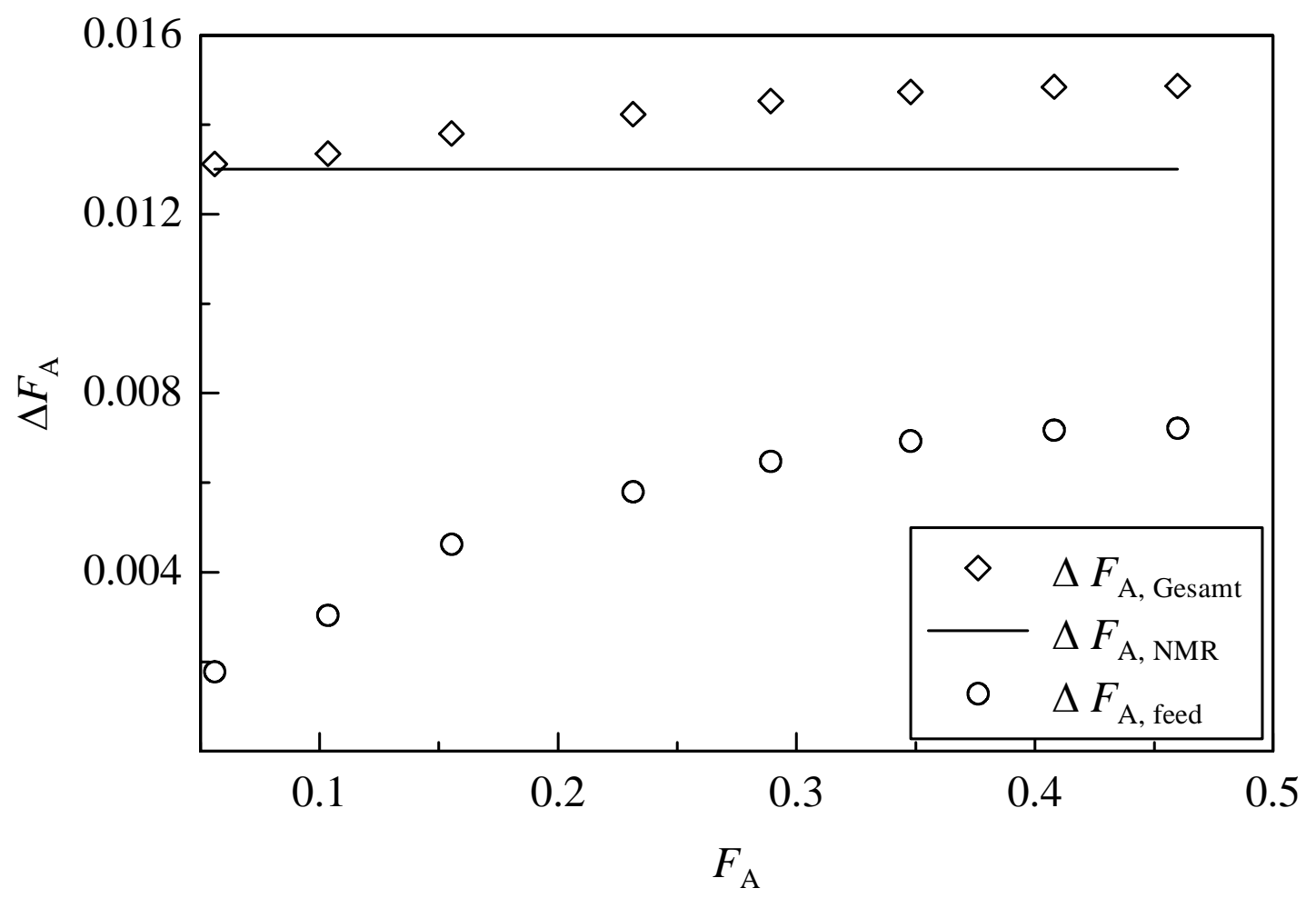

Abbildung 4.2: Verlauf des Fehlers der Copolymerzusammensetzung, $\Delta F_{\mathrm{A}}$ sowie der Teilfehler $\Delta F_{\mathrm{A}, N M R}, \Delta F_{\mathrm{A}, \text { feed }}$ mit $F_{\mathrm{A}}$ am Beispiel einer Ethen-EthylacrylatCopolymerisation bei $170^{\circ} \mathrm{C}$ und 2000 bar

Der aus der Unsicherheit der Comonomerzusammensetzung resultierende Fehler der Copolymerzusammensetzung $\Delta F_{\mathrm{A} \text {, feed }}$ nimmt mit $F_{\mathrm{A}}$ zu. Es deutet sich aber an, dass die Größe dieses Fehlers zu höherem $F_{\mathrm{A}}$ auf einen konstanten Wert ausläuft. Der Gesamtfehler von $F_{\mathrm{A}}$ nimmt mit der Zusammensetzung nur in geringem Umfang $\mathrm{zu}$ und wird maßgeblich von dem (konstanten) Fehler der Zusammensetzungsanalyse bestimmt. 


\subsection{Bestimmung von Copolymerisationsparametern - Ergebnisse}

Im folgenden Abschnitt werden die für die untersuchten Ethen-(Meth)Acrylat-Systeme erhaltenen Copolymerisationsparameter vorgestellt.

\subsubsection{Ethen-Acrylat-Copolymersysteme}

Im Unterschied zu Studien über die auch großtechnisch hergestellten Ethen-Copolymere mit Methyl- bzw. Butylacrylat sind bisher $f_{\mathrm{A}}-F_{\mathrm{A}}$-Daten für die Systeme Ethen-Ethylacrylat und Ethen-Propylacrylat in der Literatur nicht mitgeteilt worden.

Im Rahmen der vorliegenden Arbeit wurden Copolymerisationen von Ethen mit Ethylacrylat bei fünf Temperaturen $\left(170,185,200,220\right.$ und $\left.240^{\circ} \mathrm{C}\right)$ und von Ethen mit Propylacrylat bei drei Temperaturen $\left(170,200\right.$ und $\left.220^{\circ} \mathrm{C}\right)$ im kontinuierlich betriebenen Rührkesselreaktor bei 2000 bar ausgeführt. Der Gesamtumsatz betrug bei allen Ethen-(Meth)Acrylat-Copolymerisationen maximal ca. $10 \%$ (vgl. Abschnitt 3.1.3), beim überwiegenden Teil weniger als $5 \%$. Es sei daran erinnert, dass der erzielte Umsatz die Auswertung mit der differentiellen Copolymerisationsgleichung (Gl. 4.14) nicht beeinträchtigt, da die Synthesen unter stationären Reaktionsbedingungen ausgeführt werden und der Umsatz bei der Ermittlung der Comonomerzusammensetzung berücksichtigt wird (vgl. Gl. 4.16).

In Abbildung 4.3 ist die Abhängigkeit der Copolymerzusammensetzung $F_{\mathrm{A}}$ von der Zusammensetzung der Monomermischung $f_{\mathrm{A}}$ für die genannten Systeme aufgetragen (LewisMayo-Plots). Aus den Grafiken ist zu erkennen, dass das jeweilige Acrylatmonomer entsprechend den Beobachtungen bei anderen Ethen-(Meth)Acrylat-Copolymerisationen stark bevorzugt eingebaut wird. Die Ausprägung dieses Effektes wird zu niedrigeren Temperaturen hin stärker. Mit zunehmender Synthesetemperatur wird der jeweils untersuchte Bereich der Copolymerzusammensetzung kleiner. Dies liegt daran, dass mit der Temperatur auch das Ausmaß der Selbstinitiierung zunimmt, sodass bei höheren Temperaturen schon bei entsprechend geringerem (Meth)Acrylatgehalt in der Reaktionsmischung der gewählte Maximalumsatz erreicht wird (vgl. Abschnitt 3.1.3). Nur bei der jeweils niedrigsten Synthesetemperatur $\left(170^{\circ} \mathrm{C}\right)$ konnte der (Meth)Acrylat-Dosierfluss so weit erhöht werden, bis die maximale Kapazität des Nachverdichters für flüssige Medien erreicht ist (vgl. Abschnitt 3.1.3).

Die Copolymerisationsparameter $r_{\mathrm{A}}$ und $r_{\mathrm{E}}$ wurden durch nichtlineare Anpassung der differentiellen Copolymerisationsgleichung (Gl. 4.14) an die $F_{\mathrm{A}}-f_{\mathrm{A}}$-Datensätze ermittelt, wobei die individuellen Fehler von $F_{\mathrm{A}}$ und $f_{\mathrm{A}}$ zur Gewichtung der Daten berücksichtigt wurden (s. Abschnitt 4.4). Wie in Abschnitt 4.2 diskutiert, wurden die Daten für eine bessere 
Vergleichbarkeit lediglich bis $\mathrm{zu}$ einem $f_{\mathrm{A}}$-Wert von 0.038 angepasst. Das Ergebnis der Anpassung ist in den Auftragungen jeweils als durchgezogene Linie dargestellt. Der weitere Verlauf des Fits über den für die Anpassung verwendeten $f_{\mathrm{A}}$-Bereich hinaus ist gestrichelt gezeichnet. Die optimalen Copolymerisationsparameter $r_{\mathrm{A}}$ und $r_{\mathrm{E}}$ sind mit den jeweils berechneten Konfidenzintervallen (JCIs, Signifikanzniveau $95 \%$ ) in den oberen Teilen der Auftragungen in Abbildung 4.3 dargestellt. Es zeigt sich, dass die JCIs mit zunehmender Temperatur eine erheblich größere Ausdehnung annehmen. Die mit der Temperatur zunehmende Unsicherheit der ermittelten $r$-Werte resultiert daraus, dass der untersuchte Zusammensetzungsbereich für höhere Temperaturen schmaler (s.o.) und die Anzahl von $F_{\mathrm{A}^{-}}$ $f_{\mathrm{A}}$-Messwertepaaren geringer ist. Die Fehlerellipsen beinhalten für das E-EA-System bei 220 und $240^{\circ} \mathrm{C}$ und für E-PA bei 200 und $220^{\circ} \mathrm{C}$ auch (physikalische unsinnige) negative Werte für $r_{\mathrm{A}}$. Die Ellipsen überlappen sich jedoch nicht. Dies bedeutet, dass die experimentelle Genauigkeit ausreicht, um die Temperaturabhängigkeit der Copolymerisationsparameter für beide Systeme eindeutig angeben zu können. Die Copolymerisationsparameter für beide Systeme sind zusammen mit ihren Standardabweichungen, welche als Schätzwerte aus den äußeren Grenzen der JCIs erhalten werden, in Tabelle 4.1 aufgelistet. Bei der Bestimmung der Standardabweichungen wird angenommen, dass die Abweichungen um die optimalen $r$-Werte einer Gauss-Verteilung gehorchen.

\begin{tabular}{ccccc}
\hline \hline & \multicolumn{2}{c}{ Ethen-Ethylacrylat } & \multicolumn{2}{c}{ Ethen-Propylacrylat } \\
\hline \hline$T /{ }^{\circ} \mathrm{C}$ & $r_{\mathrm{A}}$ & $r_{\mathrm{E}}$ & $r_{\mathrm{A}}$ & $r_{\mathrm{E}}$ \\
\hline 170 & $6.9 \pm 0.9$ & $0.041 \pm 0.002$ & $7.5 \pm 0.9$ & $0.045 \pm 0.002$ \\
185 & $6.2 \pm 1.1$ & $0.049 \pm 0.002$ & n. best. & n. best. \\
200 & $5.4 \pm 1.1$ & $0.056 \pm 0.002$ & $6.0 \pm 3.6$ & $0.063 \pm 0.006$ \\
220 & $4.0 \pm 2.4$ & $0.067 \pm 0.004$ & $4.2 \pm 2.8$ & $0.071 \pm 0.005$ \\
240 & $3.8 \pm 6.4$ & $0.080 \pm 0.010$ & n. best. & n. best. \\
\hline
\end{tabular}

Tabelle 4.1: Copolymerisationsparameter $r_{\mathrm{E}}$ und $r_{\mathrm{A}}$ für Ethen-Ethylacrylat- bzw. EthenPropylacrylat-Copolymerisationen bei 2000 bar im CSTR. 

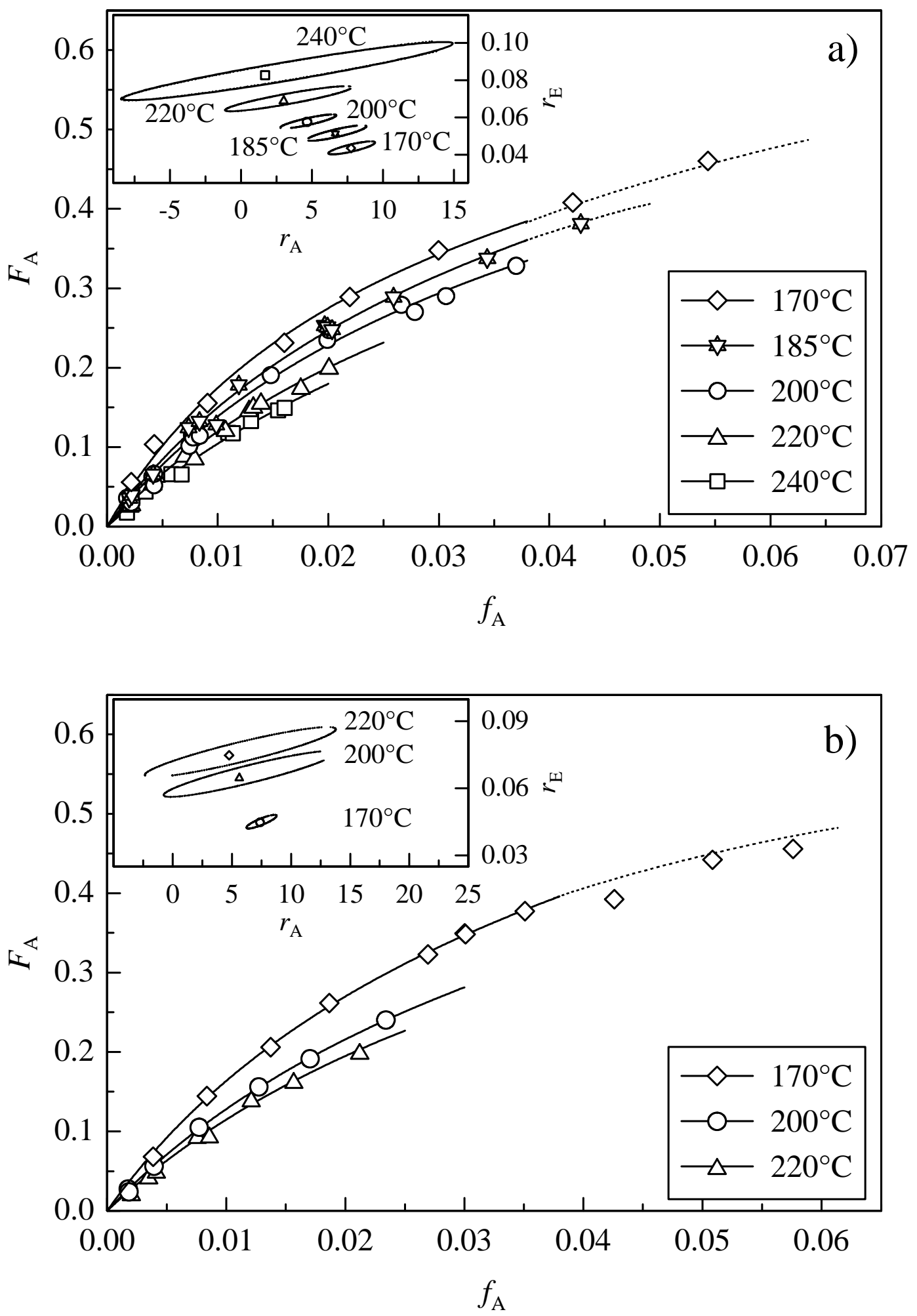

Abbildung 4.3: Abhängigkeit des Acrylatanteils im Copolymer $F_{\mathrm{A}}$ vom Acrylatanteil in der Monomermischung $f_{\mathrm{A}}$ für Copolymerisationen bei 2000 bar von Ethen mit: a) Ethylacrylat bei 170, 185, 200, 220 und $240^{\circ} \mathrm{C}$, b) Propylacrylat bei 170, 200 und $220^{\circ} \mathrm{C}$. Die Anpassung der Daten erfolgt durch nichtlineare Regression nach Gleichung 4.14. Im oberen Teil der Abbildungen sind die erhaltenen Copolymerisationsparameter sowie die berechneten JCIs bei den angegebenen Temperaturen dargestellt. 
Zur Verdeutlichung der Temperaturabhängigkeit der für die Systeme E-EA und E-PA erhaltenen Copolymerisationsparameter sind die Werte in Abbildung 4.4 in Form einer Arrheniusauftragung dargestellt. Die Daten für E-EA und E-PA sind hierbei als offene Symbole gekennzeichnet. Die zum Vergleich mit in die Auftragung eingezeichneten Literaturergebnisse für E-MA [20] sind als gefüllte Symbole dargestellt.

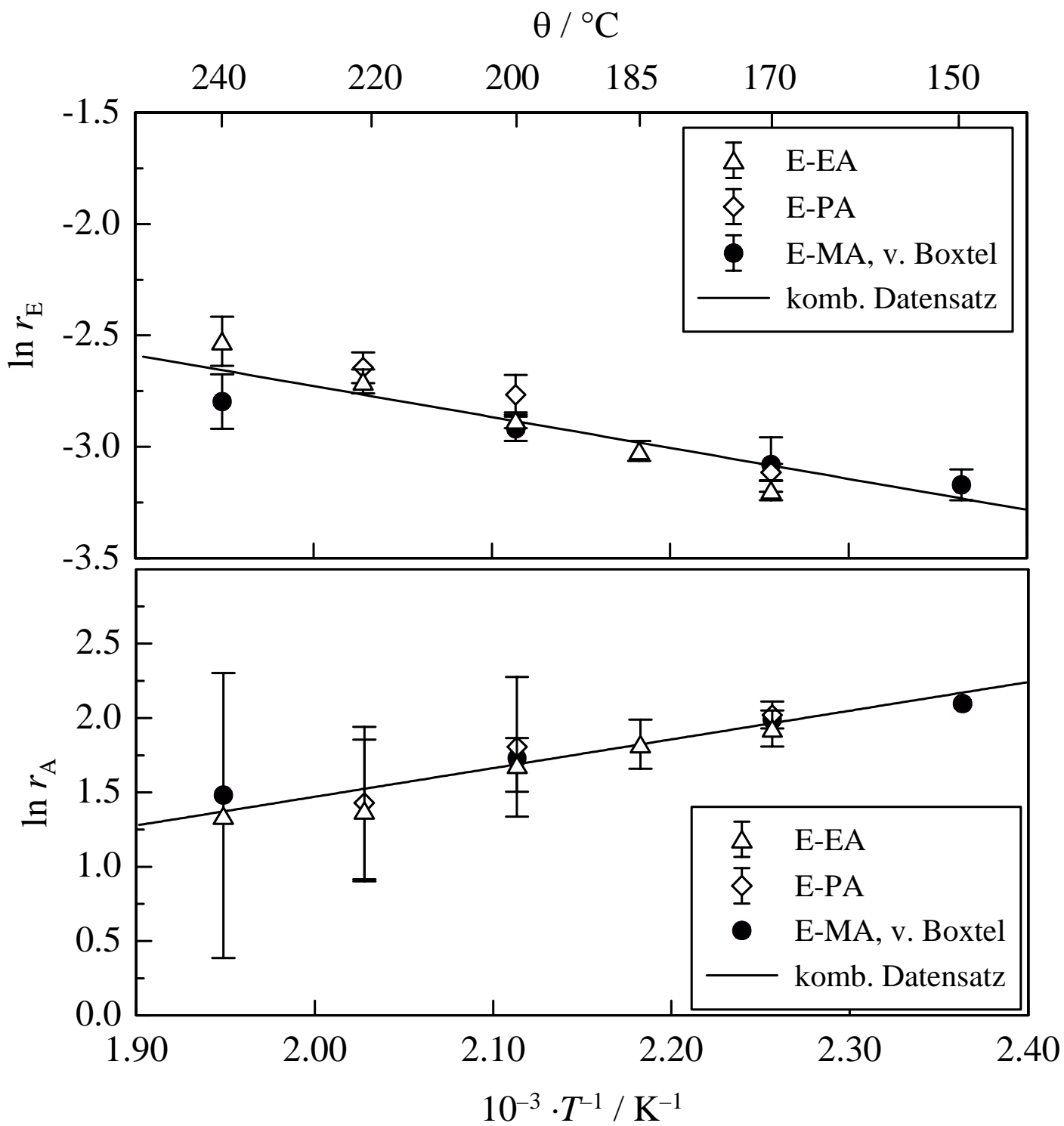

Abbildung 4.4: Temperaturabhängigkeit der Copolymerisationsparameter $r_{\mathrm{A}}$ und $r_{\mathrm{E}}$ für Ethen-Ethylacrylat- und Ethen-Propylacrylat-Copolymerisationen im Vergleich zu Literaturwerten für E-MA von v. Boxtel [20]. Die eingezeichneten Linien entsprechen den Regressionsgeraden für den Ausgleich des kombinierten Datensatzes.

Aus den Arrheniusplots ist ersichtlich, dass das Copolymerisationsverhalten von Methyl-, Ethyl- und Propylacrylat mit Ethen nicht unterschieden werden kann. Bei gegebener 
Temperatur sind die $r_{\mathrm{A}}$-Werte für E-MA, E-EA und E-PA innerhalb der Messgenauigkeit nahezu deckungsgleich. Lediglich der für E-EA für $240^{\circ} \mathrm{C}$ ermittelte $r_{\mathrm{E}}$-Wert zeigt auch unter Berücksichtigung der jeweiligen Unsicherheit eine geringe Abweichung zu höheren Werten.

Unter Annahme der Gültigkeit der Arrheniusbeziehung $\ln r_{\mathrm{A} / \mathrm{E}}=\ln A-E_{\mathrm{A}} /(\mathrm{R} \cdot T(\mathrm{~K}))$ für die Temperaturabhängigkeit der Copolymerisationsparameter lassen sich die jeweiligen Aktivierungsenergien $E_{\mathrm{A}}$ und präexponentiellen Faktoren A berechnen. Aufgrund der guten Übereinstimmung der Ergebnisse mit den Literaturdaten für E-MA wurde ein gemeinsamer Ausgleich des Datensatzes vorgenommen. Die in Abbildung 4.4 jeweils als durchgezogene Linie dargestellten Arrheniusgeraden wurden durch nichtlineare Regression der $r$ - $T$-Daten berechnet, wobei die aus der JCI-Berechnung erhaltenen geschätzten Standardabweichung der $r$-Werte als individuelle Fehler verwendet werden. Für die $r$-Werte des E-MA-Systems werden die in der Literatur angegebenen Fehler berücksichtigt. Aus dem Ausgleich wird für $r_{\mathrm{E}}$ eine Aktivierungsenergie von $11.5 \mathrm{~kJ} \cdot \mathrm{mol}^{-1}$ und ein präexponentieller Faktor von 1.05 erhalten. Für die Aktivierungsenergie von $r_{\mathrm{A}}$ berechnet sich ein Wert von $-15.0 \mathrm{~kJ} \cdot \mathrm{mol}^{-1}$ bei einem präexponentiellen Faktor von $9.19 \cdot 10^{-2}$. Es ist anzumerken, dass die aus dem gemeinsamen Ausgleich ermittelten Aktivierungsenergien etwas größere Werte annehmen als die Ergebnisse die in der Literatur [20] für die alleinige Auswertung des Systems E-MA mitgeteilt werden.

Während der Ethen-Copolymerisationsparameter $r_{\mathrm{E}}$ sich im untersuchten Temperaturbereich mit vergleichsweise hoher Genauigkeit bestimmen lässt, zeigt sich an den bei 220 und $240^{\circ} \mathrm{C}$ als auch noch für E-PA bei $200^{\circ} \mathrm{C}$ breiten Fehlerbalken, dass es schwierig ist exakte $r_{\mathrm{A}}$-Werte für höhere Temperaturen anzugeben. Die Ursache hierfür liegt darin, dass prinzipiell die Sensitivität des $r_{\mathrm{A}}$-Werts bei der Bestimmung von Copolymerisationsparametern mit der Lewis-Mayo-Gleichung (Ausdruck 4.14) mit sinkendem Comonomergehalt in der Monomermischung abnimmt. Für eine präzise Bestimmung der Copolymerisationsparameter sollte wenn möglich der gesamte Bereich der Copolymerzusammensetzung für die Auswertung herangezogen werden. Wie erwähnt, konnten in dieser Arbeit bei höheren Temperaturen aus technischen Gründen jedoch nur ethenreiche Copolymere hergestellt werden. Der mit zunehmender Temperatur stärker eingeschränkte Zusammensetzungsbereich der untersuchten Copolymere (s.o.) vermindert die Präzision der $r_{\mathrm{A}}$-Bestimmung. Angesichts der erheblichen Unsicherheit in den $r_{\mathrm{A}}$-Werten (vgl. Abbildung 4.4) ist es bemerkenswert, wie gut sich alle für ein Copolymersystem bestimmten Werte auf einer Ausgleichsgerade anordnen. 


\subsubsection{Ethen-Methacrylat-Copolymersysteme}

Aus der Literatur sind an Untersuchungen zur Copolymerisationskinetik von Systemen des Typs Ethen-Methacrylat bisher nur Arbeiten zu Ethen-Methylmethacrylat- und EthenButylmethacrylat-Copolymeren bekannt [23,31]. In dieser Arbeit wurde ergänzend das System Ethen-Ethylmethacrylat studiert. Außerdem wurden Copolymere von Ethen mit Methylmethacrylat bzw. Butylmethacrylat für thermodynamische Untersuchungen hergestellt. Anhand der Ergebnisse der Zusammensetzungsanalyse dieser Systeme können die Literaturdaten überprüft werden. Es sei in diesem Zusammenhang erwähnt, dass die Literaturdaten $[23,31]$ für Synthesen in einem weniger idealen Reaktor (CTR) erhalten wurden. Zudem erschien es interessant, als Beispiel einer Ethen-Copolymerisation mit einem funktionalisierten Methacrylatmonomer das System Ethen-Benzylmethacrylat (E-BzMA) zu untersuchen.

Es wurden bei jeweils drei Temperaturen Copolymerisationen von Ethen mit Methylmethacrylat $\left(170,200\right.$ und $\left.240^{\circ} \mathrm{C}\right)$ und mit Ethylmethacrylat $\left(180,200,220^{\circ} \mathrm{C}\right)$ durchgeführt. EBMA und E-BzMA-Copolymere wurden lediglich bei einer Temperatur $\left(200^{\circ} \mathrm{C}\right)$ hergestellt. Die Vorgehensweisen bei den Synthesen und bei der Auswertung sind identisch mit denjenigen, die für die Ethen-Acrylat-Systeme angewandt wurde (s.o.).

In Abbildung 4.5 ist die Abhängigkeit der Copolymerzusammensetzung $F_{\mathrm{A}}$ von der Zusammensetzung der Monomermischung $f_{\mathrm{A}}$ für die vier betrachteten Ethen-Methacrylat-Systeme dargestellt. Die abgebildeten Linien stellen die Resultate der Anpassung der Daten mittels nichtlinearer Regression nach Gleichung 4.17 dar. In den jeweils oberen Teilen der Auftragungen in Abbildung 4.5 sind die unter Berücksichtigung der individuellen Fehler erhaltenen Copolymerisationsparameter und die zugehörigen Konfidenzintervalle (95\% Signifikanzniveau) dargestellt. Für die beiden Ethen-Methacrylat-Systeme E-MMA und E-EMA wird keine Überlappung der für unterschiedliche Temperaturen erhaltenen JCIs beobachtet, sodass eine eindeutige Temperaturabhängigkeit der Copolymerisationsparameter ermittelt werden kann. 

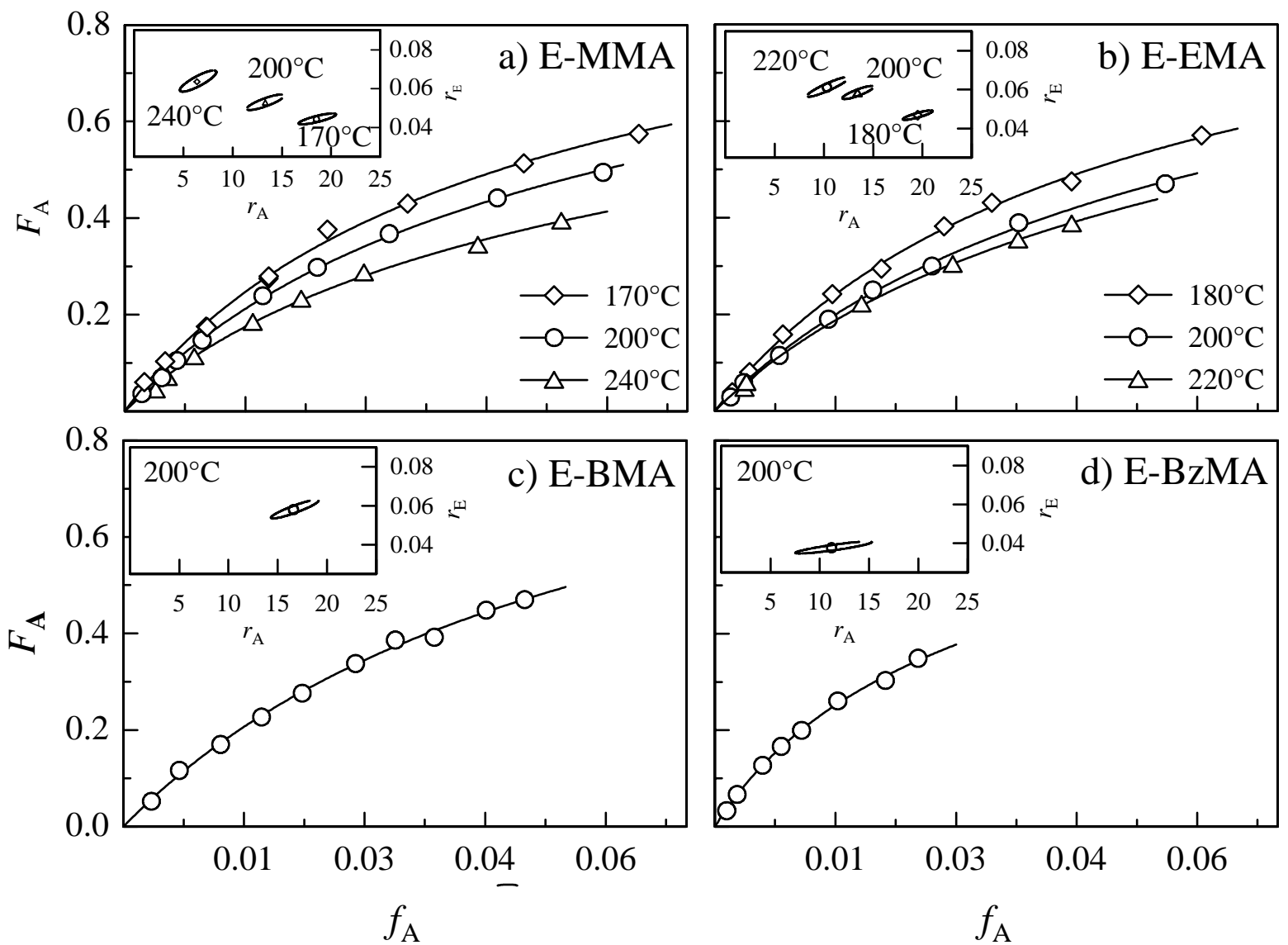

Abbildung 4.5: Abhängigkeit des Methacrylatanteils im Copolymer $F_{\mathrm{A}}$ vom Methacrylatanteil in der Monomermischung $f_{\mathrm{A}}$ für Ethen-Copolymerisationen bei 2000 bar im CSTR mit: a) Methylmethacrylat bei 170, 200 und $240^{\circ} \mathrm{C}$, b) Ethylmethacrylat bei 180, 200 und $220^{\circ} \mathrm{C}$ c) Butylmethacrylat bei $200^{\circ} \mathrm{C}$, d) Benzylmethacrylat bei $200^{\circ} \mathrm{C}$. Die Anpassung der Daten erfolgte mittels nichtlinearer Regression nach Gleichung 4.17. Im oberen Teil der Abbildungen sind die Copolymerisationsparameter und ihre JCIs bei den angegebenen Temperaturen dargestellt.

Die erhaltenen $r$-Werte sowie die aus den Arrheniusauftragungen ermittelten Aktivierungsenergien und präexponentiellen Faktoren sind in Tabelle 4.2 zusammengestellt. Die Temperaturabhängigkeit der Copolymerisationsparameter $r_{\mathrm{A}}$ und $r_{\mathrm{E}}$ für E-MMA und E-EMA wird in Abbildung 4.6 verdeutlicht. Die für $200^{\circ} \mathrm{C}$ für E-BMA ermittelten $r$-Werte sind zu Vergleichszwecken in die Arrheniusauftragungen miteingezeichnet. Für die Systeme E-MMA und E-EMA wurden durch nichtlineare Regression der $r$ - $T$-Daten die Aktivierungsenergien $E_{\mathrm{A}}$ und die präexponentiellen Faktoren A berechnet, wobei die aus der JCI-Berechnung erhaltenen geschätzten Standardabweichungen der $r$-Werte als individuelle Fehler angewandt wurden. 


\begin{tabular}{|c|c|c|c|c|}
\hline \multirow[b]{2}{*}{$T /{ }^{\circ} \mathrm{C}$} & \multicolumn{2}{|c|}{ Ethen-Methylmethacrylat } & \multicolumn{2}{|c|}{ Ethen-Ethylmethacrylat } \\
\hline & $r_{\mathrm{A}}$ & $r_{\mathrm{E}}$ & $r_{\mathrm{A}}$ & $r_{\mathrm{E}}$ \\
\hline 170 & $18.5 \pm 1.0$ & $0.044 \pm 0.003$ & n. best & n. best \\
\hline 180 & n. best. & n. best & $19.5 \pm 0.8$ & $0.047 \pm 0.003$ \\
\hline 200 & $13.3 \pm 1.0$ & $0.053 \pm 0.003$ & $13.4 \pm 0.9$ & $0.058 \pm 0.003$ \\
\hline 220 & n. best. & n.best. & $10.3 \pm 1.1$ & $0.061 \pm 0.003$ \\
\hline 240 & $6.4 \pm 1.0$ & $0.064 \pm 0.004$ & n. best. & n. best. \\
\hline $\begin{array}{c}E_{\mathrm{A}} / \\
\mathrm{kJ} \cdot \mathrm{mol}^{-1}\end{array}$ & $\begin{array}{r}-25.5^{+3.1} \\
-3.1 \\
\end{array}$ & $9.8 \begin{array}{r}+2.3 \\
-2.3\end{array}$ & $\begin{array}{r}31.8^{+3.4} \\
-3.5 \\
\end{array}$ & $\begin{array}{r}12.5^{+3.2} \\
-2.9 \\
\end{array}$ \\
\hline \multirow[t]{2}{*}{ A } & $1.86 \cdot 10^{-2}$ & 0.631 & $4.23 \cdot 10^{-3}$ & 1.32 \\
\hline & \multicolumn{2}{|c|}{ Ethen-Butylmethacrylat } & \multicolumn{2}{|c|}{ Ethen-Benzylmethacrylat } \\
\hline$T /{ }^{\circ} \mathrm{C}$ & $r_{\mathrm{A}}$ & $r_{\mathrm{E}}$ & $r_{\mathrm{A}}$ & $r_{\mathrm{E}}$ \\
\hline 200 & $16.6 \pm 1.3$ & $0.058 \pm 0.003$ & $11.1 \pm 2.5$ & $0.038 \pm 0.002$ \\
\hline
\end{tabular}

Tabelle 4.2: Copolymerisationsparameter $r_{\mathrm{E}}$ und $r_{\mathrm{A}}$ für Ethen-Methylmethacrylat-, EthenEthylmethacrylat-, Ethen-Butylmethacrylat- und Ethen-BenzylmethacrylatCopolymerisationen bei 2000 bar im CSTR. Die Zahlenwerte für die Aktivierungsenergie $E_{\mathrm{A}}$ und den präexponentiellen Faktor A beziehen sich auf die Gleichung $\ln r=\ln A-\left(E_{\mathrm{A}} /(R(T / K))\right.$. Als Unsicherheit in $E_{\mathrm{A}}$ ist die geschätzte Standardabweichung, wie sie aus der nichtlinearen $r_{\mathrm{E} / \mathrm{A}}-T$-Anpassung erhalten wird, angegeben.

Die bei getrennter Auswertung der Datensätze ermittelten Aktivierungsenergien für $r_{\mathrm{E}}$ und $r_{\mathrm{A}}$ und präexponentiellen Faktoren sind in Tabelle 4.2 angegeben. Berücksichtigt man die Unsicherheit von ca. $\pm 3.5 \mathrm{~kJ} \cdot \mathrm{mol}^{-1}$, können die Systeme bezüglich der Aktivierungsenergien nicht unterschieden werden. Aus Abbildung 4.6 ist ersichtlich, dass sich die Temperaturabhängigkeit sowohl der $r_{\mathrm{A}^{-}}$als auch der $r_{\mathrm{E}^{-}}$Werte von E-MMA und E-EMA sehr gut mit einer gemeinsamen Arrheniusgeraden beschreiben lässt, welche bei Berücksichtigung der Unsicherheit auch den jeweiligen Datenpunkt für E-BMA befriedigend wiedergibt. Aus dem gemeinsamen Ausgleich berechnet sich eine Aktivierungsenergie für $r_{\mathrm{A}}$ und $r_{\mathrm{E}}$ von -29.5 bzw. $10.3 \mathrm{~kJ} \cdot \mathrm{mol}^{-1}$. Die dazugehörigen präexponentiellen Faktoren betragen $7.31 \cdot 10^{-3}$ (für $r_{\mathrm{A}}$ ) und 0.743 (für $r_{\mathrm{E}}$ ). 


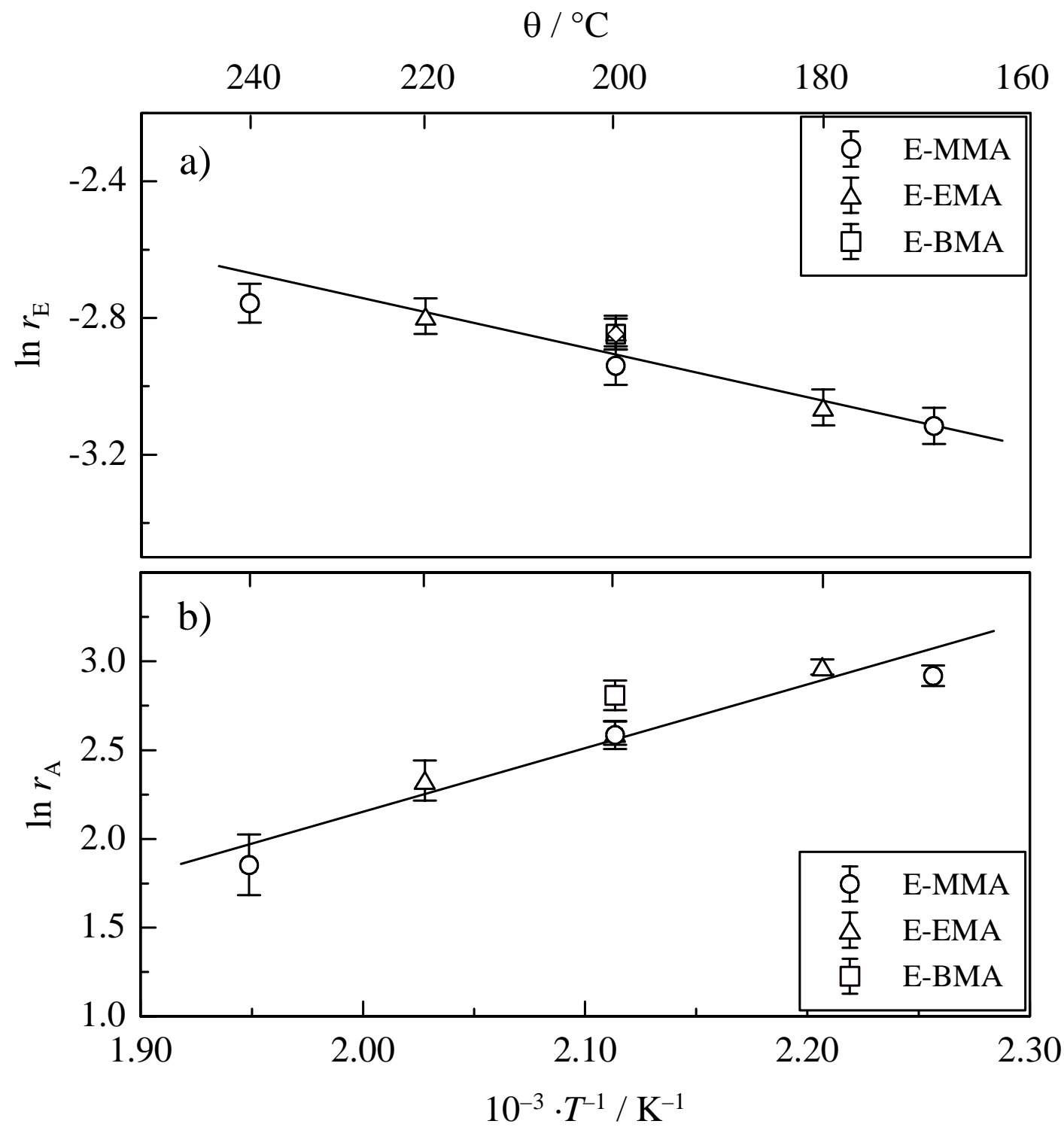

Abbildung 4.6: Temperaturabhängigkeit der Copolymerisationsparameter $r_{\mathrm{E}}(a)$ und $r_{\mathrm{A}}(b)$ für Ethen-Methacrylat-Copolymerisationen bei 2000 bar im CSTR. Dargestellt sind Daten für die Systeme Ethen-Methylmethacrylat (E-MMA), Ethen-Ethylmethacrylat (E-EMA) und Ethen-Butylmethacrylat (E-BMA)). Die Arrheniusgeraden berücksichtigen den kombinierten Datensatz.

Es wird vorgeschlagen, für die Berechnung von $r$-Werten für die Ethen-MethacrylatCopolymere E-MMA, E-EMA, E-BMA die Aktivierungsenergien und den präexponentiellen Faktor aus dem Ausgleich des kombinierten Datensatzes zu verwenden (s. Abschnitt 4.6).

Ergänzend sei erwähnt, dass der für das untersuchte funktionalisierte E-Methacrylat-System, E-BzMA erhaltene $r_{\mathrm{A}^{-}}$Wert auf dieser Ausgleichsgerade liegt. Der für E-BzMA bestimmte $r_{\mathrm{E}^{-}}$ Wert liegt jedoch deutlich niedriger. 


\subsection{Ethen-(Meth)Acrylat-Copolymerisationsparameter- Vergleich mit Literaturdaten}

Aufgrund der z.T. hohen Unsicherheit der bestimmten Copolymerisationsparameter ist es vorteilhaft für einen Vergleich des Copolymerisationverhaltens der verschiedenen (Meth)Acrylate untereinander und mit Literaturdaten zunächst die primären experimentellen Ergebnisse $\left(F_{\mathrm{A}}-f_{\mathrm{A}}-\right.$ Wertepaare $)$ zu betrachten.

In Abbildung 4.7 werden in Form von Lewis-Mayo-Auftragungen die in dieser Arbeit für die Ethen-Acrylat-Systeme E-EA und E-PA sowie die für die Ethen-Methacrylat-Systeme EMMA, E-EMA und E-BMA erhaltenen Ergebnisse für vier verschiedene Temperaturen (170, 200, 220 und $240^{\circ} \mathrm{C}$ ) dargestellt. Zum Vergleich sind Literaturdaten für E-MA, E-BA, EMMA und E-BMA mit eingezeichnet. In den Auftragungen ist der jeweils aus der LewisMayo-Anpassung (s.o.) für das E-MMA-System erhaltene Fit als durchgezogene Linie gezeigt. Zur Verdeutlichung des Unterschieds zwischen Ethen-Acrylat- und Ethen-Methacrylat-Systemen sind die Fitkurven für das E-MA-System gestrichelt dargestellt.

Aus den Auftragungen für $170^{\circ} \mathrm{C}$ und $200^{\circ} \mathrm{C}$ ist zunächst ersichtlich, dass die Daten für die Systeme E-EA und E-PA untereinander nicht unterscheidbar sind und außerdem sehr gut mit den Literaturdaten für E-MA übereinstimmen. Weiterhin findet sich eine gute Übereinstimmung mit den als offene Quadrate gezeichneten Literaturdaten für E-BA. Für diese ist analog zu den E-EA- und E-PA-Wertepaaren - die Copolymerzusammensetzung ${ }^{1} \mathrm{H}-\mathrm{NMR}$ spektroskopisch ermittelt worden. In Form kariert ausgefüllter Quadrate sind zum Vergleich Ergebnisse derselben E-BA-Copolymerisationsexperimente dargestellt, wobei hierbei jedoch die Bestimmung der Zusammensetzung anhand von ${ }^{13} \mathrm{C}$-NMR-Spektren ausgeführt wurde. Hier und auch in der Auftragung für $220^{\circ} \mathrm{C}$ zeigt sich, dass mit dieser Methode bei höherem Comonomergehalt der Copolymere niedrigere Werte für den BA-Gehalt im Copolymer ermittelt werden. Die deutliche Abweichung des jeweils höchsten Datenpunkt vom typischen Verlauf der Lewis-Mayo-Kurve deutet darauf hin, dass die ${ }^{13} \mathrm{C}-\mathrm{NMR}$-Analyse für E-BACopolymere bei höheren Gehalten zu systematischen Abweichungen führt. In der Auftragung für $220^{\circ} \mathrm{C}$ wird für die Systeme E-EA und E-PA ebenso wie für E-EA bei $240^{\circ} \mathrm{C} \mathrm{im} \mathrm{Vergleich}$ zu den Literaturdaten für E-MA ein weniger steiler Verlauf der Copolymerzusammensetzung mit der Zusammensetzung der Comonomermischung beobachtet. Auch für das System E-BA wird bei beiden Temperaturen jeweils ein flacherer Verlauf beobachtet. Der Unterschied zum E-MA-System ist für E-BA etwas geringer. Die Abweichungen der verschiedenen E-AcrylatCopolymersysteme voneinander sind unter Berücksichtigung der Unsicherheit der Daten jedoch nicht signifikant. 
Vergleicht man das Copolymerisationsverhalten der verschiedenen Ethen-MethacrylatSysteme miteinander, so ist aus der Auftragung für $200^{\circ} \mathrm{C}$ ersichtlich, dass für diese Systeme ebenfalls kein Unterschied durch die verschieden großen Esterseitenketten von E-MMA, EEMA und E-BMA entsteht. Dies zeigt sich auch anhand des Graphen für $220^{\circ} \mathrm{C}$. Aus diesem ist gut erkennbar, dass die im Rahmen dieser Arbeit erhaltenen experimentellen Daten für das System E-EMA sehr gut mit den Literaturdaten für E-BMA und E-MMA übereinstimmen.

Weiterhin machen die Auftragungen deutlich, dass bis zu einer Copolymerzusammensetzung von ca. 25 mol-\% das Copolymerisationsverhalten der Ethen-Acrylat-Systeme nicht von dem der Ethen-Methacrylat-Systeme unterschieden werden kann. Zu größeren Werten für den (Meth)Acrylatgehalt der Copolymere ergeben sich bei 170 und $200^{\circ} \mathrm{C}$ allerdings deutliche Unterschiede. Der Verlauf der Copolymerzusammensetzung mit der Zusammensetzung der Comonomermischung ist für die Ethen-Methacrylatsysteme steiler. Bei den E-MethacrylatSystemen wird im Vergleich zu den Ethen-Acrylat-Systemen bei gleicher Zusammensetzung der Comonomermischung während der Reaktion ein größerer Comonomeranteil ins Copolymer eingebaut. Der Unterschied zwischen beiden Copolymersystem-Familien nimmt mit der Temperatur ab. Während bei $170^{\circ} \mathrm{C}$ der Unterschied in der Copolymerzusammensetzung noch bis zu ca. 16 mol-\% beträgt, finden sich bei $220^{\circ} \mathrm{C}$ (mit Ausnahme eines einzelnen Datenpunkts für E-BA) bereits keine signifikante Differenzen. Bei $240^{\circ} \mathrm{C}$ lässt sich praktisch kein Unterschied mehr feststellen, wobei anzumerken ist, dass für Ethen-Acrylat-Copolymere im Bereich hoher $f_{\mathrm{A}}$-Werte keine experimentellen Daten vorhanden sind und nur vorausberechnete Copolymerzusammensetzungen (dargestellt durch die gestrichelte Linie) für die Gegenüberstellung zur Verfügung stehen. 


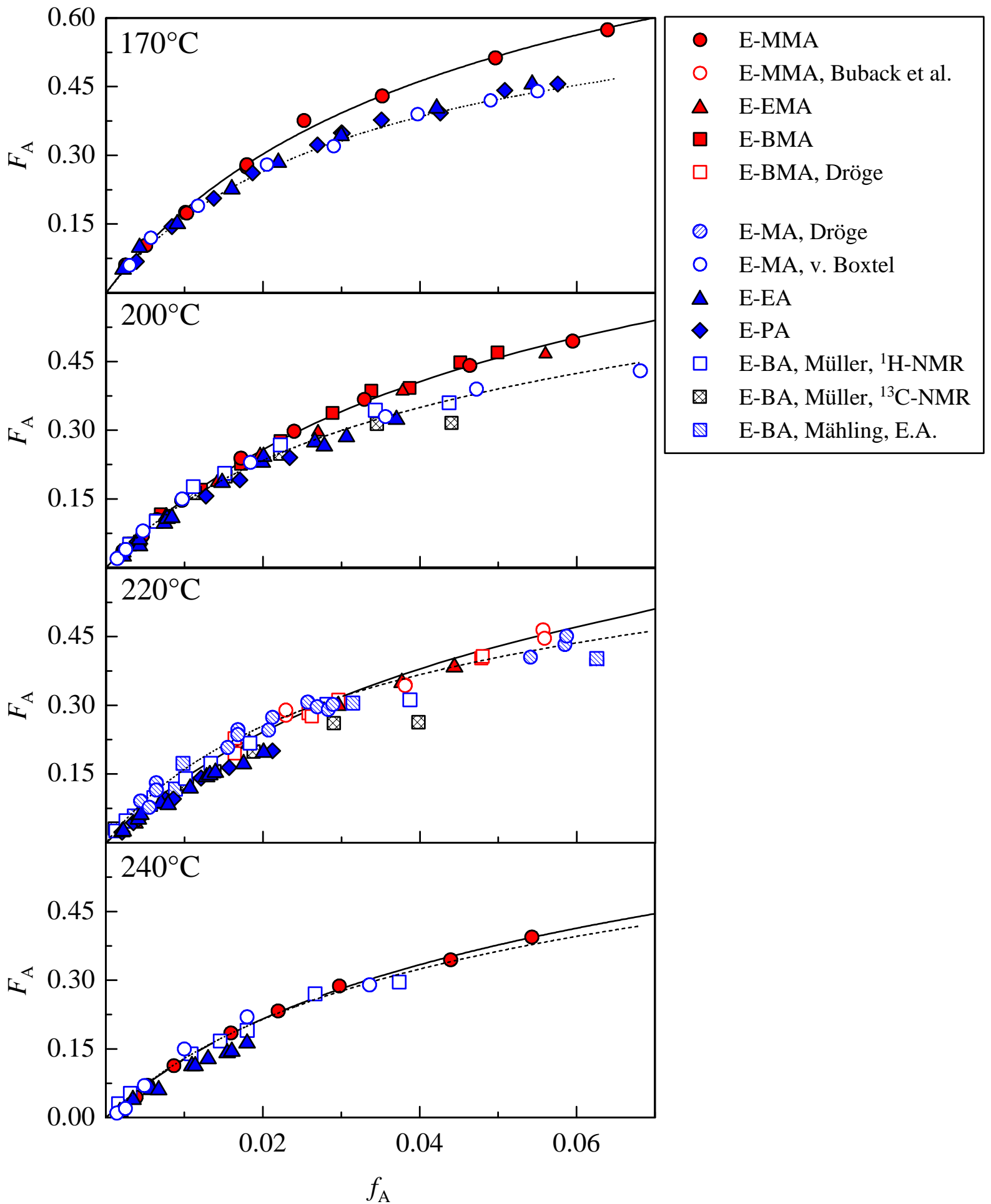

Abbildung 4.7: Abhängigkeit des (Meth)Acrylatanteils im Copolymer $F_{\mathrm{A}}$ vom (Meth)Acrylatanteil in der Monomermischung $f_{\mathrm{A}}$ für Ethen-Copolymerisationen bei 2000 bar im CSTR mit verschiedenen (Meth)Acrylaten. Dargestellt sind Ergebnisse für 170, 200, 220 und $240^{\circ} \mathrm{C}$. Die durchgezogene Linien zeigen die Anpassungen der E-MMA-Daten nach Gleichung 4.14. Die gestrichelte Linie entspricht dem Ergebnis der Anpassung für E-MA. Dargestellt sind Ergebnisse dieser Arbeit und Literaturdaten für E-MA [31, 20], für E-BA von [34, 33], für E-MMA [23] und für E-BMA [31]. 
In Abbildung 4.8 ist die Temperaturabhängigkeit der in der vorliegenden Arbeit erhaltenen $r$ Werte für die Systeme E-EA, E-PA, E-MMA, E-EMA und E-BMA im Vergleich zu den bisher verfügbaren Literaturdaten für Ethen-(Meth)Acrylat-Copolymerisationen dargestellt. Der überwiegende Teil der Ergebnisse beruht auf Copolymerisationen im kontinuierlichen Rührkessel. Lediglich für die Literaturdaten von E-MMA und E-BMA fand ein ungerührter, kontinuierlich betriebener Kessel Anwendung.

Die blau dargestellten Symbole stehen für die $r$-Werte der Ethen-Acrylat-Systeme. Die Daten für Ethen-Methacrylat-Systeme sind als rot markierte Symbole gezeichnet, wobei die in dieser Arbeit erhaltenen Messwerte gefüllt und die Literaturdaten offen bzw. schraffiert dargestellt sind. Beim Vergleich der $r_{\mathrm{E}}$-Werte (Abb. 4.8 a) wird deutlich, dass die Daten der bisher untersuchten E-(Meth)Acrylat-Systeme im gesamten untersuchten Temperaturbereich nur geringfügig voneinander abweichen. Es lässt sich weder ein signifikanter Unterschied zwischen den Werten für die Ethen-Methacrylat- und denen der Ethen-Acrylat-Systeme, noch ein Trend mit der Länge der Esteralkoholgruppe der (Meth)Acrylate feststellen. Es erscheint daher gerechtfertigt, die Temperaturabhängigkeit der $r_{\mathrm{E}}$-Werte für alle Systeme mit einer gemeinsamen Arrheniusgerade zu beschreiben. Das Resultat dieses Vorgehens wird in Abbildung 4.8 a) in Form einer durchgezogenen, den kombinierten Datensatz berücksichtigenden Linie illustriert. Den größten Unterschied zu dieser Ausgleichsgerade weisen die $r_{\mathrm{E}}$-Werten für E-MA und EBMA von Dröge [31] auf. Zur Erklärung ist zu erwähnen, dass in der Studie von Dröge nur sehr wenig Copolymerproben in dem für den $r_{\mathrm{E}}$-Wert sensitiven Bereich geringer (Meth)Acrylatgehalte untersucht wurden. Es ist anzunehmen, dass die von Dröge bestimmten $r_{\mathrm{E}}$ Werte im Vergleich zu denen von v. Boxtel, Müller und dieser Arbeit eine höhere Unsicherheit aufweisen. 


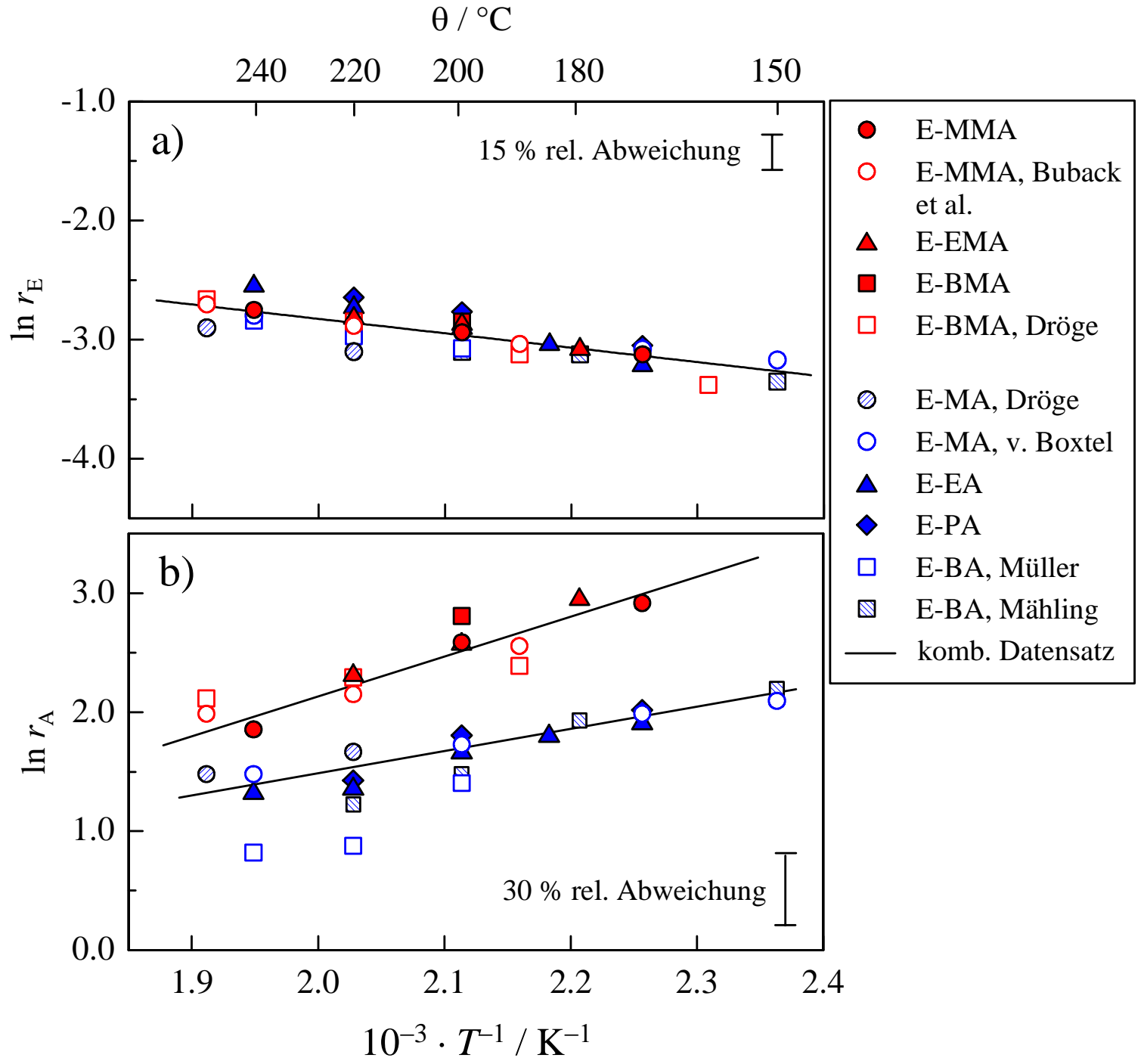

Abbildung 4.8: Copolymerisationsparameter $r_{\mathrm{E}}(a)$ und $r_{\mathrm{A}}$ (b) für Ethen-(Meth)AcrylatCopolymerisationen bei 2000 bar. Dargestellt sind Daten für die Systeme EEA, E-PA, E-MMA, E-EMA und E-BMA im Vergleich zu Literaturdaten für E-MA [31] und [20], für E-BA [34] und [33], für E-MMA [23] und für E$B M A[31]$.

In der Literatur wurde gezeigt, dass die Copolymerisationsparameter für einige Copolymersysteme wie Acrylnitril-Acrylat [35] und Styrol enthaltende Systeme [36] gut mit den Quotienten der Geschwindigkeitskoeffizienten der Addition von, mit den entsprechenden Kettenenden vergleichbaren, kleinen Radikalen und den jeweiligen Monomeren übereinstimmen. Dies begründet sich damit, dass das Kettenwachstum in der radikalischen Polymerisation ein chemisch kontrollierter Vorgang ist, sodass die zugehörige Aktivierungsenergie nicht wesentlich von der Größe des wachsenden Radikals abhängt. Der üblicherweise beobachtete Unterschied im präexponentiellen Faktor des Additiongeschwindigkeitskoeffizienten eines kleinen Radikalmoleküls im Vergleich zu demjenigen für ein polymeres 
Radikal wird durch die Verhältnisbildung bei der Berechnung des „Modell“-Copolymerisationsparameters kompensiert. Es ist zu erwähnen, dass für diese Ableitung die Gültigkeit des Terminalmodells angenommen wird. Für die Abschätzung von $r_{\mathrm{E}}$-Werten für die in der vorliegenden Arbeit untersuchten Systeme müsste die Addition von primären Alkylradikalen an die Monomere Ethen und (Meth)Acrylat betrachtet werden. Da hierzu bislang aus der Literatur keine Daten bekannt sind, werden im Folgenden Werte für die Additionsreaktion von Methylradikalen verwendet. In Tabelle 4.3 sind die von Fischer und Radom [37] publizierten Aktivierungsenergien für die Addition des Methylradikals, des $t$ - $\mathrm{Bu}-\mathrm{O}-\mathrm{CO}-\mathrm{CH}_{2}-$ Radikals und des $t$-Bu-O-CO-C $\left(\mathrm{CH}_{3}\right)_{2}$-Radikals an verschiedene Momomere (im Lösungsmittel Acetonitril) aufgelistet. Der kursiv dargestellte Wert für die Addition von $t$-Bu-O-CO$\mathrm{CH}_{2} \bullet$ an Ethen kann über eine Abschätzung erhalten werden [23].

\begin{tabular}{|c|c|c|}
\hline \multicolumn{2}{|l|}{ System } & \multirow{2}{*}{$\frac{E_{\mathrm{A}} / \mathrm{kJ} \mathrm{mol}^{-1}}{28.2 \pm 3}$} \\
\hline $\mathrm{CH}_{3} \bullet$ & + Ethen & \\
\hline & $+\mathrm{MMA}$ & $16.0 \pm 3$ \\
\hline & $+\mathrm{MA}$ & $16.9 \pm 3$ \\
\hline \multirow[t]{2}{*}{$t-\mathrm{Bu}-\mathrm{O}-\mathrm{CO}-\mathrm{CH}_{2} \bullet$} & + Ethen & $24.8 \pm 3$ \\
\hline & $+\mathrm{MA}$ & $15.6 \pm 3$ \\
\hline \multirow[t]{2}{*}{$t-\mathrm{Bu}-\mathrm{O}-\mathrm{CO}-\mathrm{C}\left(\mathrm{CH}_{3}\right)_{2} \bullet$} & + Ethen & $38.2 \pm 4$ \\
\hline & + MMA & $22.4 \pm 3$ \\
\hline
\end{tabular}

Tabelle 4.3: $\quad$ Aktivierungsenergien für die Additionsreaktion des Methylradikals, des $t$-Bu$\mathrm{O}-\mathrm{CO}-\mathrm{CH}_{2}$-Radikals (tert-butoxycarbonylmethyl-) und des $t$-Bu-O-CO$\mathrm{C}\left(\mathrm{CH}_{3}\right)_{2}$-Radikals (2-tert-butoxycarbonyl-2-propyl-) mit verschiedenen Monomeren (im Lösungsmittel Acetonitril) aus Ref. [37]. 
Die abgeschätzten Aktivierungsenergien der $r$-Werte errechnen sich aus der Differenz der Aktivierungsenergie der Addition der „Homoaddition“ zur derjenigen für die „Kreuzaddition":

$$
E_{\mathrm{A}}\left(r_{\mathrm{i}}\right)=E_{\mathrm{A}}\left(k_{\mathrm{p}}, \mathrm{ii}\right)-E_{\mathrm{A}}\left(k_{\mathrm{p}, \mathrm{ij}}\right)
$$

Mit den Werten für die Additionsreaktion des Methylradikals an Ethen $\left(28.2 \mathrm{~kJ} \cdot \mathrm{mol}^{-1}\right)$ und an Methylacrylat $\left(16.9 \mathrm{~kJ} \cdot \mathrm{mol}^{-1}\right)$ bzw. an Methylmethacrylat $\left(16.0 \mathrm{~kJ} \cdot \mathrm{mol}^{-1}\right)$ aus [37] erhält man mit Gleichung 4.30 eine Aktivierungsenergie für den Copolymerisationsparameter $r_{\mathrm{E}}$ der Ethen-Methylacrylat-Copolymerisation von $11.3 \pm 3 \mathrm{~kJ} \cdot \mathrm{mol}^{-1} \mathrm{bzw}$. für denjenigen der EthenMethylmethacrylat-Copolymerisation von $12.2 \pm 3 \mathrm{~kJ} \cdot \mathrm{mol}^{-1}$. Diese Werte stimmen sehr gut mit der Aktivierungsenergie von $E_{\mathrm{A}}=10.0 \mathrm{~kJ} \cdot \mathrm{mol}^{-1}$ überein, welche sich aus dem gemeinsamen Ausgleich der experimentellen Daten ergibt (vgl. Abbildung 4.8 a)). Anzumerken ist hierbei, dass die Abschätzungen aus den kinetischen Koeffizienten für kleine Radikale entsprechend den experimentellen Beobachtung keinen signifikanten Unterschied bezüglich der Temperaturabhängigkeit der $r_{\mathrm{E}}$-Werte für MA und MMA vorhersagen.

Die Beurteilung der in Abbildung 4.8 b) dargestellten Temperaturabhängigkeit der $r_{\mathrm{A}}$-Werte der verschiedenen Ethen-Copolymersysteme ist erheblich schwieriger als jene für $r_{\mathrm{E}}$ da die $r_{\mathrm{A}}$-Werte eine wesentlich höhere Unsicherheit aufweisen. Dies verdeutlicht sich an den exemplarisch für die durchschnittliche Unsicherheit in 4.8 a) und b) eingezeichneten Fehlerbalken. Die Ursache für die Unsicherheit in $r_{\mathrm{A}}$ liegt primär darin, dass aus technischen Gründen (vgl. Abschnitt 4.5.1) Ethen-Copolymersysteme bisher nur in einem stark eingeschränkten $f_{\mathrm{A}}$-Bereich untersucht wurden. Bei der Bestimmung der $r_{\mathrm{A}}$-Werte ist jedoch gerade der nicht untersuchte, (meth)acrylatreiche Bereich der Comonomermischung derjenige mit der höchsten Sensitivität. Wie aus Abbildung 4.7 erkennbar, wurden nahezu alle Copolymerisationen bei einem (Meth)Acrylatanteil von weniger als 6 mol-\% in der Comonomermischung durchgeführt. Entsprechend zeigt sich in Abbildung $4.8 \mathrm{~b}$ ) eine deutliche Streuung der $r_{\mathrm{A}}$-Werte für die verschiedenen Copolymersysteme.

Im Folgenden werden zunächst die Ethen-Acrylat-Systeme betrachtet. Für die aus verschiedenen Literaturquellen $[20,31]$ stammenden $r_{\mathrm{A}}$-Werte des E-MA-Systems ergibt sich in guter Näherung eine lineare Temperaturabhängigkeit. Die Ergebnisse für die Ethen-Copolymere mit Acrylaten mit längerer Esterseitenkette stimmen bis etwa $200^{\circ} \mathrm{C}$ sehr gut mit den $r$ Werten für E-MA überein. Die $r$-Werte bei 220 und $240^{\circ} \mathrm{C}$ liegen für E-EA, E-PA gering- 
fügig und für E-BA im Vergleich zu E-MA deutlich niedriger. Anhand der in Abbildung 4.7 aufgetragenen (primären) experimentellen Daten für die verschiedenen Ethen-AcrylatCopolymersysteme, welche auch bei 220 und $240^{\circ} \mathrm{C}$ zumindest im untersuchten $f_{\mathrm{A}}$-Bereich praktisch nicht unterschieden werden können, wird deutlich, dass die in den Arrheniusauftragungen (4.8 b) erkennbaren Abweichungen der verschiedenen Systeme wahrscheinlich allein aus der Ungenauigkeit der Bestimmungsmethode resultieren. Es sei erwähnt, dass eine Erklärung für die sich möglicherweise andeutende Abweichung der Copolymersysteme mit längerer Alkylestergruppe auf den bei Acrylat-Polymerisationen mit der Temperatur zunehmend auftretenden Verzweigungen [38] beruht. Es ist vorstellbar, dass die sich durch „Backbiting“ und/oder Transfer auf das Polymer bildenden tertiären Radikale die Wachstumskinetik beeinflussen.

Die erwähnte Unsicherheit der erhaltenen $r_{\mathrm{A}}$-Werte führt dazu, dass die Berechnung der Aktivierungsenergie aus einem gemeinsamen Ausgleich der Ergebnisse aller Ethen-Acrylatsysteme nur zu einer formalen Größe führt. Es wird vorgeschlagen, bei einem Ausgleich die Daten für E-BA aufgrund ihrer besonders großen, offensichtlich systematischen Abweichung bei einem gemeinsamen Ausgleich nicht zu berücksichtigen. Die unter dieser Voraussetzung erhaltene Ausgleichsgerade ist in Abbildung 4.8 b) als durchgezogene Linie eingezeichnet. Die zugehörige Aktivierungsenergie beträgt $-15.0 \mathrm{~kJ} \cdot \mathrm{mol}^{-1}$ (s.a. Abschnitt 4.5.1).

Möchte man aus kinetischen Daten für Additionsreaktionen kleiner Radikale - wie voranstehend für den $r_{\mathrm{E}}$-Wert erläutert - einen ,theoretischen“ $r_{\mathrm{A}}$-Wert bestimmen, so tritt das Problem auf, dass Werte für Reaktionen von (Meth)Acrylat-Radikalen mit Ethen bzw. mit (Meth)Acrylaten in der Literatur bisher nicht mitgeteilt wurden. Für das MethylacrylatRadikal kann als Modellsubstanz in guter Näherung das „MEst“-Radikal (tert-butoxycarbonylmethyl) verwendet werden. Die für die Addition des „MEst“-Radikal an MA bzw. Ethen ermittelte Aktivierungsenergie beträgt $15.6 \mathrm{~kJ} \cdot \mathrm{mol}^{-1}$ bzw. $24.8 \mathrm{~kJ} \cdot \mathrm{mol}^{-1}$ [37]. Für die Aktivierungsenergie von $r_{\mathrm{A}}$ der Ethen-Methylacrylat-Copolymerisation lässt sich somit ein Wert von $-9.2 \pm 4 \mathrm{~kJ} \cdot \mathrm{mol}^{-1}$ errechnen. Dieser Wert stimmt innerhalb der Unsicherheit mit dem aus dem gemeinsamen Ausgleich der experimentellen $r_{\mathrm{A}}$-Werte für E-MA, E-EA und EPA erhaltenen Aktivierungsenergie von $-15.0 \pm 4 \mathrm{~kJ} \cdot \mathrm{mol}^{-1}$ überein.

Beim Vergleich der in dieser Arbeit erhaltenen $r_{\mathrm{A}}$-Werte für E-MMA (rot unterlegte Kreissymbole in Abbildung 4.8 b) mit Literaturdaten (rote, ungefüllte Kreissymbole) findet sich eine befriedigende Übereinstimmung. Es wurde in dieser Arbeit eine etwas größere Temperaturabhängigkeit der $r_{\mathrm{A}}$-Werte (vgl. Abschnitt 4.5.2) bestimmt. Der für E-BMA ermittelte $r_{\mathrm{A}}$-Wert weicht von den entsprechenden Literaturwerten geringfügig in Richtung 
höherer Zahlenwerte ab. Unter Berücksichtigung der experimentellen Genauigkeit ist festzustellen, dass die Daten aller drei, in verschiedenen Arbeiten untersuchten, Ethen-MethacrylatSysteme ineinander streuen und nicht unterschieden werden können. Somit kann für die Ethen-Methacrylat-Systeme analog zu den Beobachtungen bei den Ethen-Acrylat-Systemen keine Abhängigkeit der Temperaturabhängigkeit von der Größe des (Meth)AcrylatComonomers festgestellt werden. In Abbildung $4.8 \mathrm{~b}$ ist das Ergebnis eines gemeinsamen Ausgleich der bisher für Ethen-Methacrylat-Copolymerisationen erhaltenen $r_{\mathrm{A}}$-Werte in Form einer durchgezogenen Linie dargestellt. Diese Gerade entspricht einer Aktivierungsenergie von $-25.8 \mathrm{~kJ} \cdot \mathrm{mol}^{-1}$. Aus der Auftragung wird ersichtlich, dass bei niedrigen Temperaturen signifikant höhere $\ln \left(r_{\mathrm{A}}\right)$-Werten für die Ethen-Methacrylat-Systeme gegenüber denen für EAcrylat-Systeme beobachtet werden. Aufgrund der stärkeren Temperaturabhängigkeit der $r_{\mathrm{A}^{-}}$ Werte der Ethen-Methacrylat-Systeme nimmt der Unterschied der beiden Copolymersystemfamilien mit der Temperatur ab. Analog der Erkenntnisse aus Abbildung 4.7 deutet es sich an, dass bei Temperaturen oberhalb von $250^{\circ} \mathrm{C}$ der Unterschied im Copolymerisationsverhalten von Ethen-Acrylat- und Ethen-Methacrylat-Systemen verschwindet.

Für eine Abschätzung der Aktivierungsenergie des $r_{\mathrm{A}}$-Wertes für E-MMA kann das „TBCP“Radikal (2-tert-butoxycarbonyl-2-propyl) als Modell-Substanz für das MethylmethacrylatRadikal verwendet, da es mit diesem konstitutionell eng verwandt ist. Die Gültigkeit dieser Analogie wird durch die Tatsache gestützt, dass die Aktivierungsenergie der Addition von TBCP an MMA (22.4 kJ $\cdot \mathrm{mol}^{-1}$ ) [37] mit derjenigen des Homowachstumsschritt von MMA [39] perfekt übereinstimmt.

Für die Aktivierungsenergie der Addition von TBCP mit Ethen kann nach einer von Buback et al. [23] beschriebenen Abschätzung ein Wert von $38.2 \mathrm{~kJ} \cdot \mathrm{mol}^{-1}$ angenommen werden. Mit der Aktivierungsenergie der Addition von TBCP an MMA von $22.4 \mathrm{~kJ} \cdot \mathrm{mol}^{-1}$ berechnet sich damit für $r_{\mathrm{A}}$ für Ethen-Methylmethacrylat-Copolymerisationen eine Aktivierungsenergie von $-15.8 \mathrm{~kJ} \cdot \mathrm{mol}^{-1}$. Die weniger gute Übereinstimmung des abgeschätzten mit dem experimentellen Wert $\left(-25.8 \mathrm{~kJ} \cdot \mathrm{mol}^{-1}\right)$ mag an der fehlenden Berücksichtigung des Einflusses von Effekten durch Druck bzw. Lösungsmittel liegen. Eine weitere möglicherweise Fehlerquelle besteht in der Verwendung eines lediglich abgeschätzten Werts für die Aktivierungsenergie der Addition von TBCP an Ethen nach [23]. Unter Anwendung des in dieser Arbeit erhaltenen experimentellen $E_{\mathrm{A}}$-Werts für $r_{\mathrm{A}}$ sollte ein (sozusagen zurückberechneter), angesichts der starken elektronischen und sterischen Stabilisierung des MMA-Radikals realistischer Wert von $48.2 \mathrm{~kJ} \cdot \mathrm{mol}^{-1}$ für die Aktivierungsenergie der Addition von TBCP an Ethen zu erwarten sein. 
Zusammenfassend lassen sich aus den Ergebnissen dieser Arbeit und bisher mitgeteilten Literaturdaten folgende Erkenntnisse über die Copolymerisationskinetik von Ethen-(Meth)Acrylat-Systemen festhalten:

1.) Ethen-(Meth)Acrylat-Copolymerisationen im Temperaturintervall von $150-290^{\circ} \mathrm{C}$ sind gekennzeichnet durch $r_{\mathrm{A}}$-Werte größer als 1 und $r_{\mathrm{E}}$-Werte erheblich kleiner als 1 .

2.) Die $r_{\mathrm{E}}$-Werte und ihre Temperaturabhängigkeit sind für alle bisher untersuchten Ethen(Meth)Acrylat-Copolymerisationen innerhalb der erzielbaren Genauigkeit der Bestimmungsmethode identisch. Die mittlere Aktivierungsenergie von $r_{\mathrm{E}}$ für die Systeme EMA, E-EA, E-PA, E-BA, E-MMA, E-EMA, E-BMA beträgt $10 \mathrm{~kJ} \cdot \mathrm{mol}^{-1}$. Der zugehörige präexponentielle Faktor hat einen Wert von 0.649 .

3.) Die Aktivierungsenergie der $r_{\mathrm{E}}$-Werte lässt sich aus Literaturdaten zur Aktivierungsenergie der Additionsreaktion des Methylradikals an Ethen und an die Monomere Methylacrylat (MA) und Methylmethacrylat (MMA) in guter Genauigkeit vorausberechnen. $\left(11.3 \pm 3 \mathrm{~kJ} \cdot \mathrm{mol}^{-1}\right)$

4.) Die Aktivierungsenergie für $r_{\mathrm{A}}$ ist innerhalb der Ethen-Acrylat-Copolymerfamilie (EMA, E-EA, E-PA und E-BA) unter Berücksichtigung der Unsicherheit ununterscheidbar. Die mittlere Aktivierungsenergie beträgt $-15.0 \pm 4 \mathrm{~kJ} \cdot \mathrm{mol}^{-1}$.

5.) Die $r_{\mathrm{A}}$-Werte der Ethen-Methacrylat-Systeme sind bei Temperaturen kleiner als $250^{\circ} \mathrm{C}$ signifkant höher als die der Ethen-Acrylat-Systeme. Die Temperaturabhängigkeit der $r_{\mathrm{A}^{-}}$ Werte der Ethen-Methacrylat-Systeme ist erheblich stärker als die der Ethen-AcrylatSysteme.

6.) Die $r_{\mathrm{A}}$-Werte und ihre Temperaturabhängigkeit sind für die untersuchten EthenMethacrylat-Systeme (E-MMA, E-EMA und E-BMA) innerhalb der erreichbaren Genauigkeit identisch. 


\subsection{Literatur Kapitel 4}

[1] G. Odian, Principles of Polymerization, $3^{\text {rd }}$ ed., John Wiley \& Sons (1991)

[2] M. D. Lechner, K. Gehrke, E. H. Nordmeier, Makromolekulare Chemie, Birkhäuser Verlag, Basel (1993)

[3] H.-G. Elias, Makromoleküle, Hüthig\&Wepf Verlag, Heidelberg (1990)

[4] L.Wittkowski, Dissertation, Göttingen (1998)

[5] P. Becker, M. Buback, J. Sandmann, Macromol. Chem. Phys. 203 (2002) 2113

[6] F. R. Mayo, F. M. Lewis, J. AM. Chem. Soc. 66 (1944) 1954

[7] T. Alfrey, Jr., G. Goldfinger, J. Chem. Phys. 12 (1944) 205

[8] E. Merz, T. Alfrey, G. Goldfinger, J. Polym. Sci. 1 (1946) 75

[9] T. Fukuda, Y. D.-Ma, H. Inagaki, Macromolecules 18 (1985) 26

[10] D. J. T. Hill, H. O’Donell, P. W. O’Sullivan, Macromolecules 15 (1982) 960

[11] O. F. Olaj, I. Bitai, F. Hinkelmann, Makromol. Chem. 188 (1987)1689

[12] T. P. Davis, in : Polym. Sci. Part A : Polym. Chem. Vol. 39, John Wiley \& Sons (2001) 597

[13] M. Buback, A. Feldermann, C. Barner-Kowollik, Macromolecules, 34 (2001) 5439

[14] T. Fukuda, Y.-D. Ma, K. Kubo, H. Inagaki, Macromolecules 24 (1991) 370

[15] Y. D. Ma, P. S. Kubo, T. Fukuda, Macromolecules 26 (1993) 6766

[16] T. P. Davis, K. F. O’Driscoll, M. C. Piton, M. A. Winnik, Polym. Int. 24 (1991) 65

[17] S. Beuermann, D. A. Paquet Jr. J. H. McMinn, R. A. Hutchinson, Macromolecules 29 (1996) 4206

[18] M Busch, A. Wahl, Macromol. Theory Simul. 7 (1998) 217

[19] J. L. Koenig, "Chemical microstructure of Polymer Chains", John Wiley \& Sons, New York 1980

[20] H. C. M. v. Boxtel, Dissertation, Göttingen (2000)

[21] S. Bywater, Trans. Faradey Soc. 51 (1955) 1267

[22] R. A. Hutchinson, S. Beuermann, D. A. Paquet Jr., J. H. McMinn, Macromolecules 30 (1997) 3490 
[23] M. Buback, H. Dietzsch, Macromol. Chem. Phys. 7, (2001)1173

[24] T. Fukuda, K. Kubo, Y.-D. Ma, Prog. Polym. Sci. 17 (1992)

[25] T. Fukuda, Y.-D. Ma, H. Iagaki, Polym. J. 14 (1982) 705

[26] J. Barton, E. Borsig, Complexes in Free Radical Chemistry, Elsevier Amsterdam (1998)

[27] H. J. Harwood, Makromol. Chem., Macromol. Symp. 10/11 (1987) 331

[28] M. Coote, P. Davis in: Handbook of Radical Polymerization, Wiley\& Sons (2002)

[29] A. M. van Herk, J. Chem. Edu. 72 (1995) 138

[30] D. W. Behnken, J. Polym. Sci. Part A 2 (1964) 645

[31] T. Dröge, Dissertation, Göttingen (1997)

[32] M. Dube, R.A. Sanayei, K. F. Driscoll, P. M. Reilly, J. Polym.Sci.: Part A: Polym. Chem. 29 (1991) 703

[33] F.-O. Mähling, Dissertation, Göttingen (1995)

[34] M. Müller, Diplomarbeit, Göttingen (2001)

[35] J. Q.Wu. I. Beranek, H. Fischer, Helv. Chim. Acta (1995) 78194

[36] M. Walbiner, J. Q. Wu, H. Fischer, Helv. Chim. Acta (1995) 78910

[37] H. Fischer, L. Radom, Angew. Chem. Int. Ed. 40 (2001) 1340

[38] C. Plessis, G. Arzamendi, J. M. Alberdi, A. M. van Herk, J. R. Leiza, J. M. Asua, Macromol. Rapid Commun. 24 (2003) 173

[39] S. Beuermann, M. Buback, T. P. Davis, R. G. Gilbert, R. A. Hutchinson, O. F. Olaj, G. T. Russell, J. Schweer, A. M. van Herk. Macromol. Chem. Phys. 198 (1997) 1545 


\section{Untersuchungen zum Entmischungsver- halten}

\subsection{Entmischungsverhalten von Polymer-Solvens-Systemen - Phänomenologie}

In diesem Abschnitt werden die zum Verständnis der Arbeit benötigten theoretischen Grundlagen zum Entmischungsverhalten von Polymeren in überkritischen Fluiden erläutert. Zunächst wird in Abschnitt 5.1.1 auf generelle Aspekte von Phasendiagrammen binärer Mischungen eingegangen. In Abschnitt 5.1.2 werden die phänomenologischen Besonderheiten von Mischungen aus Polymer und Lösungsmitteln behandelt. Ausführliche Darstellungen der Theorie der Thermodynamik von Polymer-Solvens-Systemen finden sich in $[1,2,3]$.

\subsubsection{Phasendiagramme binärer Mischungen}

Die Beschreibung des Phasenverhaltens (quasi)binärer Mischungen erfordert eine (wenigstens) dreidimensionale Darstellung der Abhängigkeit des Drucks ( $p$ ) von der Temperatur $(T)$ und vom Stoffmengenanteil $(x)$ einer Komponente. Zusätzlich können Abhängigkeiten von der mittleren Polymermolmasse, der Molmassenverteilung und von mikrostrukturellen Charakteristika der Polymere betrachtet werden. Im Rahmen der vorliegenden Arbeit wurden Messungen des Phasenverhaltens bei konstantem Stoffmengenanteil von Polymer und Solvens durchgeführt. Daher werden im folgenden lediglich zweidimensionale $p$ vs. T-Projektionen der Raumdarstellung behandelt. Detaillierte Beschreibungen und Klassifizierungen von Phasendiagrammen von Polymer-Solvens-Systemen finden sich in der Literatur bei McHugh [2], Scott [3] und Chen und Radosz [4].

In Abbildung 5.1 wird in Diagramm a) ein generalisiertes Phasendiagramm vom Typ III [2] einer binären Mischung niedermolekularer Stoffe gezeigt. Die durchgezogenen, unbezeichneten Linien stellen jeweils die Dampfdruckkurven der Komponenten dar. Die Kurven enden in den kritischen Punkten $\mathrm{C}_{1}$ und $\mathrm{C}_{2}$. Komponente 1 ist die flüchtigere der beiden. Die gestrichelt gezeichnete, steile Kurve bei niedriger Temperatur trennt den Bereich in dem die beiden Komponenten als nicht mischbare Flüssigkeiten vorliegen (LL) vom homogenen Bereich (Fluid) ab. Diese Linie wird als upper-critical-solution-temperature curve (UCST) bezeichnet. Die sehr geringe Druckabhängigkeit des LL-Fluid-Übergangs entlang dieser Linie beruht darauf, dass die betrachteten Phasen als wenig kompressible Flüssigkeiten 
vorliegen. $\mathrm{Zu}$ geringeren Drücken trifft die UCST-Kurve auf eine mit LLV bezeichnete Linie. Unterhalb dieser liegt das System in Form zweier Flüssigkeiten und einer Dampfphase vor. Eine zweite gestrichelt dargestellte Linie beginnt bei $\mathrm{C}_{2}$ und endet auf einer weiteren LLV-Linie.

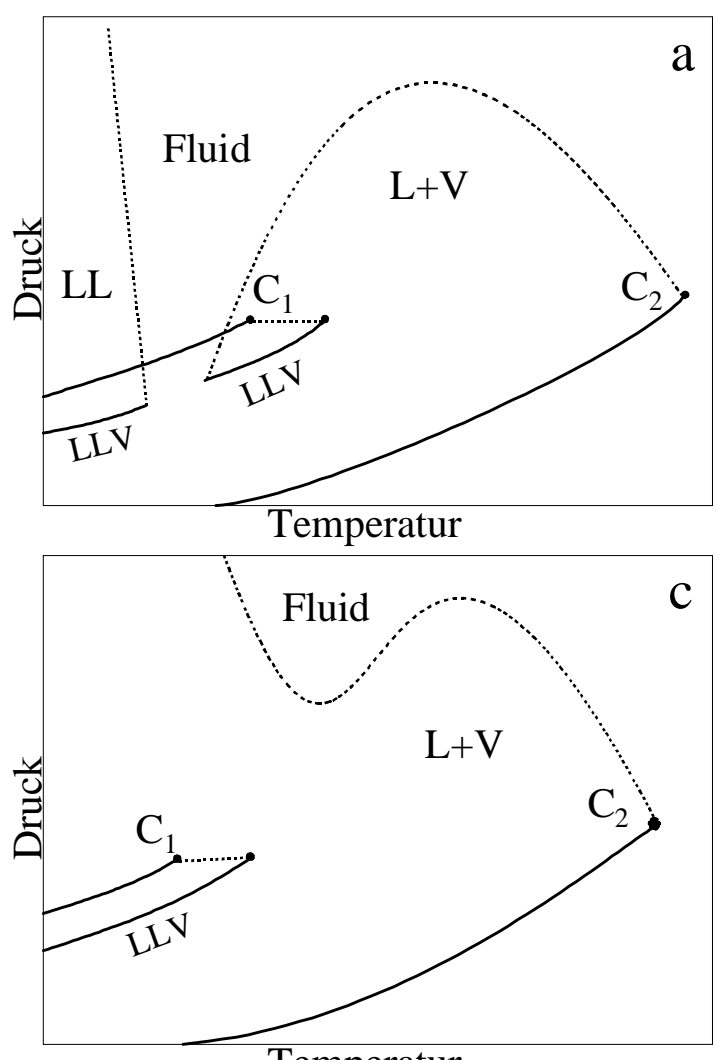

Temperatur

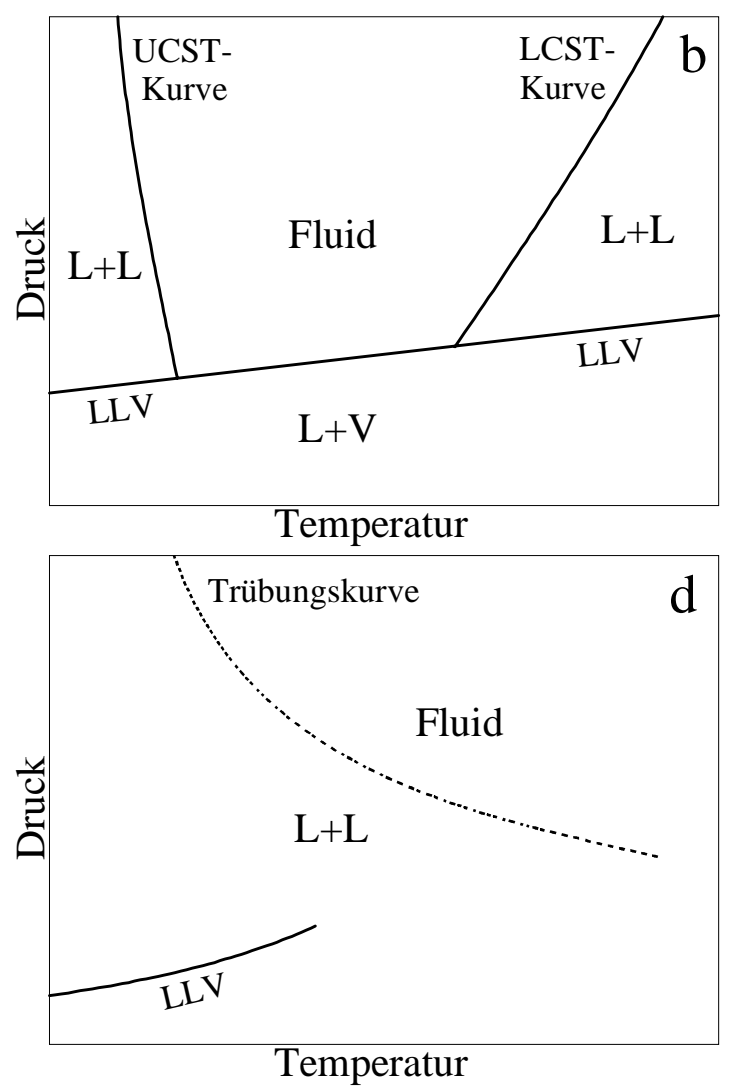

Abbildung 5.1: Schematische p/T-Diagramme von (quasi)binären Mischungen aus: a) niedermolekularen Stoffen mit Typ III-Verhalten, b) Polymer und Lösungsmittel mit Typ III-Verhalten, c) niedermolekularen Stoffen mit Typ IV-Verhalten, d) Polymer-Fluid mit Typ IV-Verhalten.

Die gestrichelte Linie ist die Grenze zwischen dem Bereich der homogenen Mischung (Fluid) und dem Bereich der Koexistenz einer flüssigen und einer Dampfphase (LV). Diese beiden Phasen besitzen in der Nähe des kritischen Punktes $C_{1}$ flüssigkeitsähnliche Dichten. Daher führt ein Anstieg der Temperatur bei konstantem Druck in diesem Bereich quasi zu einem Zerfall der homogenen Phase in zwei koexistierende flüssige Phasen. Experimentell wird dabei entlang der als lower-critical-solution-temperarture curve (LCST) bezeichneten Grenzlinie eine Eintrübung beobachtet.

Das entsprechende Phasendiagramm vom Typ III für Mischungen aus Polymer und Solvens ist in Abbildung 5.1 mit b) gekennzeichnet. Es wird deutlich, dass nur ein Ausschnitt des Diagramms a) aus dem Bereich geringer Temperaturen dargestellt ist. Dies liegt daran, dass 
sich das Polymere weit unterhalb der zu erwartenden kritischen Temperatur zersetzt. Weiterhin ist der Dampfdruck des Polymeren vernachlässigbar gering. Die LLV-Linien aus Diagramm a) (Übergang zur Koexistenz von drei Phasen) fallen für Polymerlösungen mit der Dampfdruckkurve des Lösungsmittels zusammen und bilden die durchgezogene LLVLinie. Unterhalb dieser Linie existiert in einem weiten Temperaturbereich ein Zweiphasengebiet (LV) aus Dampf und Flüssigkeit. Die gestrichelt gezeichnete UCST- und LCST-Linie trennen jeweils den homogenen Bereich (Fluid) von den aus zwei flüssigen Phasen bestehenden Bereichen $(\mathrm{L}+\mathrm{L})$ ab. Die beiden Linien stellen entgegengesetzte Phasengrenzlinien dar. Im Falle der UCST-Linie führt eine isobare Temperaturabsenkung zur Zweiphasigkeit, im Falle der LCST-Linie zur Einphasigkeit.

Für binäre Mischungen bestimmter niedermolekularer Stoffe wird z.T. ein von Diagramm a) abweichendes Phasenverhalten beobachtet. Dieses Typ IV [2] genannte Verhalten ist in Diagramm c) dargestellt. Anstelle der UCST-Kurve und der weiteren, das homogene Gebiet abgrenzenden, Kurve (siehe Diagramm a)) wird nur eine kritischen Entmischungskurve beobachtet. Diese weist oft, wie in c) dargestellt, ein Druckminimum auf.

Diagramm d) zeigt das entsprechende Typ IV-Verhalten von Polymer-Solvens-Mischungen. Für diese Systeme findet man ebenfalls nur eine kritische Entmischungs- auch als U-LCSTbezeichnete Kurve [4], welche aus der Überlagerung der UCST-, LCST- und LLV-Kurve resultiert. Typischerweise zeigen alle im Rahmen dieser Arbeit untersuchten SolvensPolymer-Mischungen ein derartiges Verhalten. Der Phasenübergang an dieser Grenzlinie findet ähnlich demjenigen an der UCST-Linie statt. Bei isobarer Absenkung der Temperatur tritt ebenso wie bei isothermer Absenkung des Drucks ein Zerfall der homogenen Fluidphase in eine polymerreiche, schwere Gelphase und eine polymerarme, leichte Solphase ein.

\subsubsection{Phasenverhalten von Mischungen aus Polymer und Solvens}

Eine Mischung aus einem Polymeren und einer niedermolekularen Komponente stellt im strengen Sinne kein binäres sondern ein multinäres System dar. Die Ursache dafür ist, dass Polymere in der Regel nicht aus einem einheitlichen Stoff bestehen, sondern polydispers d.h. aus einer Mischung verschieden großer, homologer Kettenmoleküle aufgebaut sind.

Erwähnenswert ist, dass bei vielen Polymeren neben der Polydispersität bezüglich der Kettenlänge weitere, die Mikrostruktur betreffende Eigenschaften, wie z.B. die Häufigkeiten von Verzweigungen, an der Polymerkette polydispers verteilt vorliegen. Copolymere können zudem eine Verteilung bezüglich der Häufigkeit der Comonomeranteile in verschie- 
denen Polymermolekülen aufweisen. Wenn die Anteile der verschiedenen Comonomerbausteine in allen Molekülen identisch sind, spricht man von einem chemisch einheitlichen Copolymeren.

Die Ursache für den oft komplexen Aufbau der Mikrostruktur von Polymeren liegt in der Vielzahl der während der Synthese zumeist parallel ablaufenden Reaktionen wie z.B.: das Kettenwachstum, der Transfer der radikalischen Funktion auf das Monomer bzw. das Polymer und die sogenannten Backbiting- [5], Scission- und Terminierungsreaktionen.

Im Folgenden wird lediglich die Polydispersität bezüglich der Kettenlänge berücksichtigt. Die chemisch-physikalischen Eigenschaften eines einzelnen Polymermoleküls ändern sich nur geringfügig und kontinuierlich mit der Kettenlänge. Da sich darüber hinaus die makroskopischen Eigenschaften eines Polymeren aus einem Mittel über alle Spezies ergeben, kann die Polymerkomponente einer Mischung dennoch näherungsweise als einheitlicher Stoff betrachtet werden. Man bezeichnet daher Mischungen aus einem Polymer und einem niedermolekularen Reinstoff auch als pseudo- oder quasibinär.

Unter dieser Vereinfachung ist es möglich die Phasengleichgewichte von PolymerLösungsmittel-Systemen in üblicherweise für binäre Mischungen verwendeten Diagrammtypen darzustellen. Als Polymerkonzentration wird dazu die Summe aller Polymerkomponenten aufgefasst, ungeachtet ihrer Kettenlänge. Trägt man das Entmischungsverhalten von Polymerlösungen in einem klassischen $T$ - $x$-Diagramm auf, so kann dabei keine Information über die in den einzelnen Phasen vorhandenen Molmassenverteilungen dargestellt werden. Mittels der Konstruktion von sogenannten Schattenkurven (s.u.) wird dies ermöglicht.

In der Regel beeinflusst die Polydispersität das Entmischungsverhalten von PolymerLösungsmittel-Systemen. Die Gleichgewichtsphasen zu einem Druck und einer Temperatur können bei gegebener Ausgangsverteilung des Polymers verschiedene Konzentrationen aufweisen. Dies führt dazu, dass mehr als eine Entmischungsgrenzkurve existiert. In Abbildung 5.2 ist schematisch ein generalisiertes $T$ - $x$-Diagramm einer Mischung aus Polymer und Lösungsmittel dargestellt. 


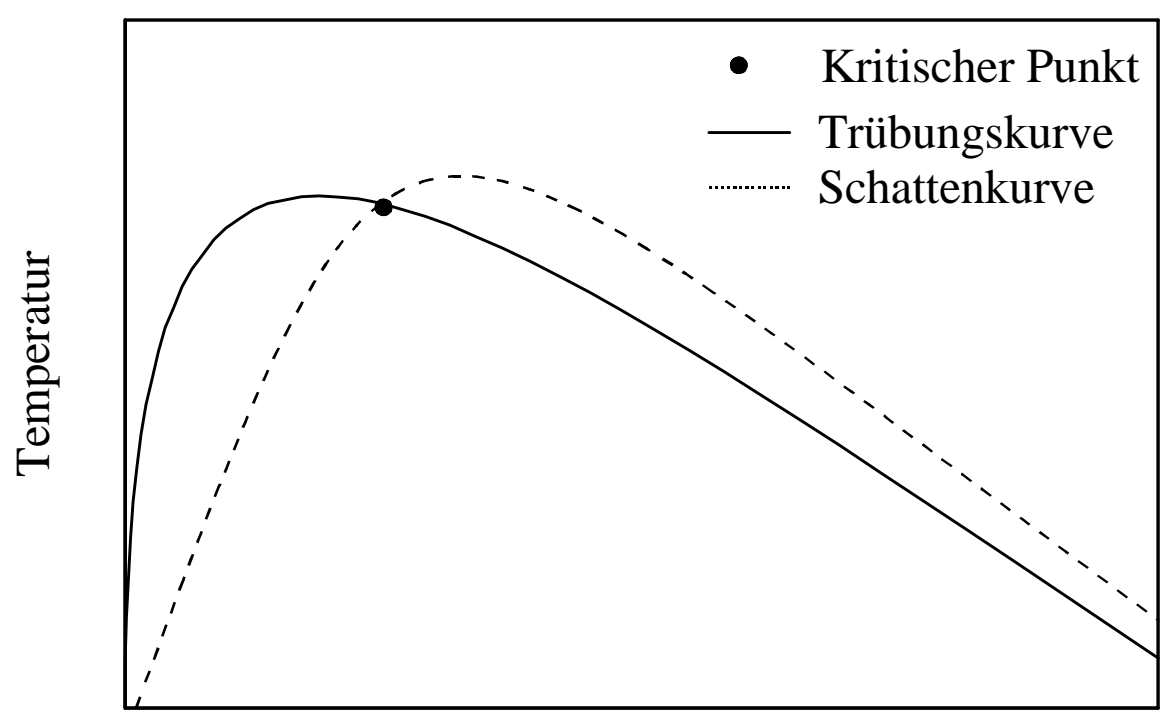

Gewichtsbruch Polymer

Abbildung 5.2: Temperatur-Gewichtsbruch-Diagramm für ein generalisiertes quasibinäres System bestehend aus einer Mischung eines polydispersen Polymers und eines Lösungsmittels.

Hierbei stehen Punkte auf der Trübungskurve (durchgezogene Linie) jeweils mit Punkten auf der Schattenkurve (gestrichelte Linie) im Gleichgewicht. Gelangt man z.B. bei gegebenen Polymergewichtsbruch durch Absenken der Temperatur aus dem homogenen Gebiet bis zur Trübungskurve, so entsteht in diesem Augenblick gerade das erste Tröpfchen einer zweiten Phase der Schattenkurve. Die Trübungsphase ist durch den Ausfall einer infinitesimalen Menge noch nicht beeinflusst, d.h. es liegt in allen Trübungspunkten gerade die Ausgangsverteilung des Polymers vor. In der Schattenphase hingegen liegt eine von der Ausgangsphase verschiedene Verteilung vor. Ist die ausfallende Phase eine polymerreiche Phase, so reichern sich in dieser im Normalfall bevorzugt längere Polymerketten an. In einer polymerarmen Phase liegen vor allem kürzere Ketten vor. Im kritischen Punkt der Lösung, welcher in quasibinären Diagrammen nicht im Extremum der Trübungskurve liegt, werden wie üblich - zwei Phasen identisch.

Ergänzend soll erwähnt werden, dass die geschilderten Besonderheiten des Entmischungsverhalten von Polymerlösungen vielfach Anwendungen in der Polymeranalytik finden. Ein Beispiel ist die fraktionierende Fällung von Polymeren nach Molmasse und Verzweigungs$\operatorname{grad}($ TREF-Technik) [6].

Die in der vorliegenden Arbeit durchgeführten Untersuchungen zum Entmischungsverhalten von Polymeren in überkritischen Fluiden konzentrieren sich auf die Bestimmung von 
Trübungskurven. Die experimentelle Bestimmung von Schattenkurven für Hochdruckphasengleichgewichte ist aufgrund der Schwierigkeit, Proben aus den koexistierenden Phasen zu nehmen, ohne das Gleichgewichts zu stören, außerordentlich aufwändig [9, 11].

\subsection{Bestimmung von Trübungsdrücken}

Die zuvor erläuterten, kritischen Entmischungskurven in den Phasendiagrammen stellen die Grenzlinien zwischen dem homogenen und dem zweiphasigen Zustandsgebiet dar. Während des Zerfalls der homogenen Phase in zwei zunächst fein verteilte koexistierende Phasen wird die ehemals transparente Mischung undurchsichtig. Die Linien werden daher auch als Trübungskurven bezeichnet.

Die Phasengrenzen bei Polymer-Solvens-Systemen sind aufgrund der Polydispersität (s.o.) im Unterschied zu Mischungen aus niedermolekularen Stoffen nicht scharf definiert. Für Polymerlösungen wird im Bereich der Phasengrenze experimentell, z.B. im Falle einer ULCST bei zunehmender isothermer Druckabsenkung, eine fortlaufende Eintrübung beobachtet.

Die experimentelle Bestimmung von Trübungspunkten von Polymer-Solvens-Systemen erfolgt üblicherweise durch visuelle Beobachtung der untersuchten Mischung in einer optischen Hochdruckzelle während einer Variation des Drucks oder der Temperatur. Um die Lage einer derartigen Phasengrenze reproduzierbar bestimmen zu können, hat es sich als vorteilhaft erwiesen, Trübungspunkte allgemein als diejenigen Punkte zu definieren, bei denen $90 \%$ des eingestrahlten Lichts absorbiert werden [7,8]. Als Trübungspunkt wird in der Regel diejenige Situation definiert, bei der Einbauten in der Messzelle, wie z.B. der Rührer, gerade nicht mehr erkannt werden können. Trübungspunkte können neben der visuellen Methode auch durch die Messung der Abschwächung von Laserlicht bestimmt werden, welche beim Durchgang durch eine Mischung mit zunehmender Eintrübung durch Streuung einsetzt [8].

Der Vorgang der zunehmenden Eintrübung wird in Abbildung 5.3 anhand einer schematischen Trübungskurve (U-LCST) verdeutlicht. Die zur Dokumentation eines Trübungsdruckexperiments an einer Mischung aus Ethen und einem Ethen-Copolymer aufgenommenen und anschließend digitalisierten Videobilder sind zur Illustration der typischen Beobachtungen während einer Messung zugeordnet. Es handelt sich um Endoskopaufnahmen des Innenraums der verwendeten Hochdruckzelle bei langsamer isothermer Absenkung des Drucks. Im unteren Teil der Bilder finden sich eingespiegelt die zuge- 
hörigen Druck- (rote Ziffern) und Temperaturmesswerte (grüne Ziffern). Auf dem obersten Bild ist die Mischung vollständig homogen und transparent. In der Bildmitte sieht man die hell erleuchtete Stirnseite des Gleitkolbens (siehe Abschnitt 3.3.2) und unten im Bild den Rührstab. Eine isotherme Druckabsenkung führt, wie auf dem zweiten Bild von oben erkennbar, zum Auftreten einer ersten Eintrübung.

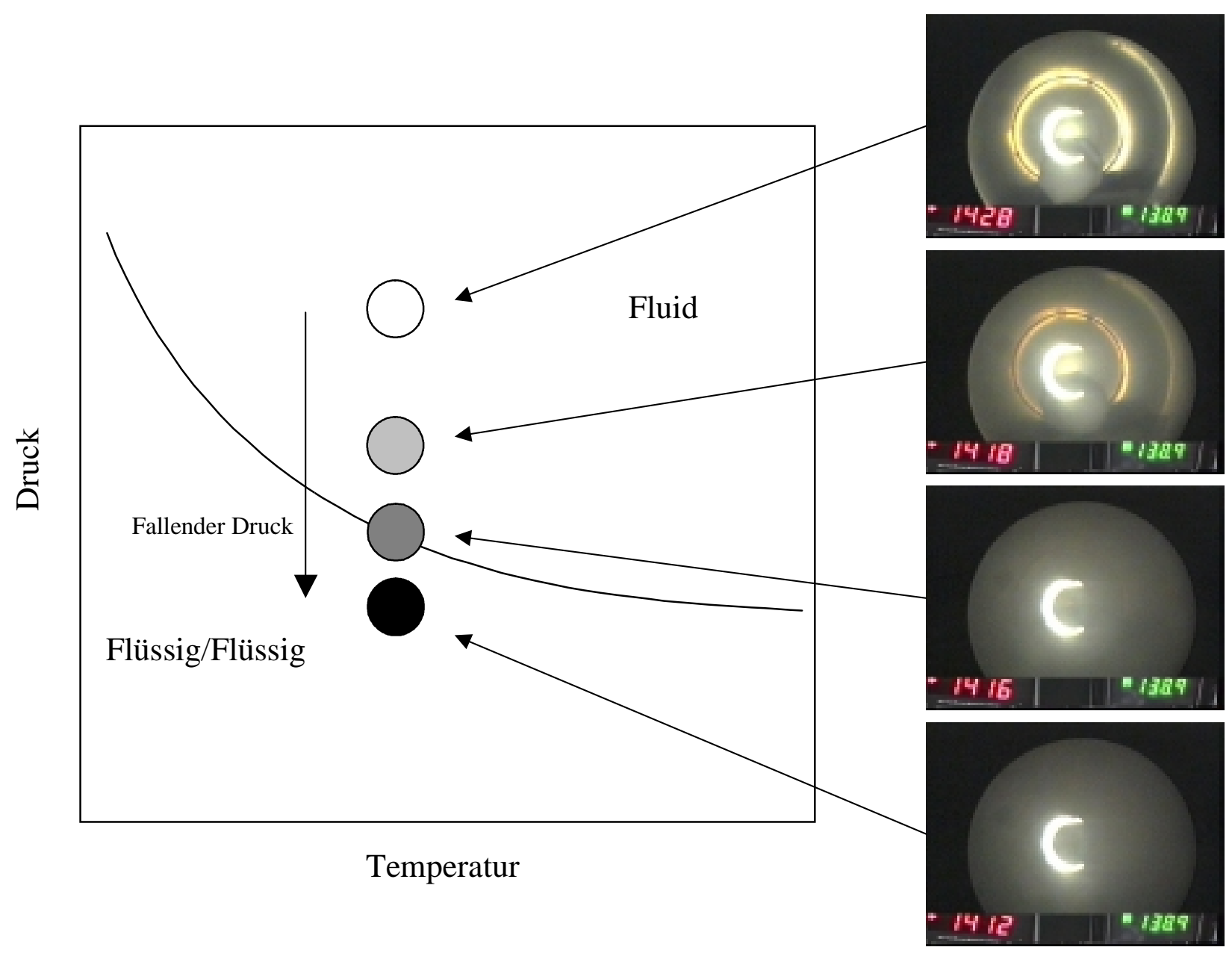

Abbildung 5.3: Schematische U-LCST-Phasengrenze (Trübungsdruckkurve) eines PolymerSolvens-Systems in einem p,T-Diagramm mit digitalisierten Videobildern einer Ethen / Polymer-Mischung während isothermer Druckabsenkung. Von oben nach unten: 1) vollständig homogene Mischung, 2) erste Eintrübung bei etwa 2 bar oberhalb des Trübungspunkts, 3) Trübungspunkt, 4) vollständig undurchsichtige Mischung.

Der Rand des Gleitkolbens ist deutlich und der Rührstab ist gerade noch zu erkennen. Eine weitere Absenkung um 2 bar führt zum Trübungspunkt (vorletztes Bild). Der Rand des Kolbens und der Rührstab sind gerade nicht mehr zu sehen. Bei weiterer Reduzierung des Drucks ergibt sich schließlich eine vollständig undurchsichtige Mischung (s. unterstes Bild). 
Es sei angemerkt, dass der helle Fleck in der Mitte der Abbildungen durch Reflexion des ein-gestrahlten Lichts am Fenster der Zelle hervorgerufen wird.

\subsection{Validierung der experimentellen Methode zur Messung von Trübungsdrücken}

In diesem Abschnitt wird die für die Trübungsdruckmessungen verwendete experimentelle Methode daraufhin überprüft, inwieweit die Ergebnisse neben dem zentralen Einfluss durch die Zusammensetzung der untersuchten Polymere von weiteren Faktoren abhängig sind. Es wird - besonders auch in Hinblick auf eine nachfolgende Modellierung der Messdaten detailliert auf mögliche systematische Fehler aufgrund unvollständiger Stabilität der Mischungen eingegangen. Außerdem wird die Beeinflussung der Messdaten durch die Verwendung von Inhibitor und durch die Polymerkonzentration diskutiert.

\subsubsection{Stabilität von Mischungen aus fluidem Ethen und Polymeren}

Die im Rahmen dieser Arbeit untersuchten Systeme sind potentiell reaktiv, so dass neben der Prüfung der Reproduzierbarkeit untersucht werden muss, ob eine Zeitabhängigkeit der gemessenen Trübungsdrücke vorliegt. Dies kann besonders gut getestet werden, indem die Messungen bei aufsteigender und absteigender Temperatur durchgeführt werden. Es sei daran erinnert, dass die bei den Trübungspunktmessungen herrschenden Bedingungen von Druck, Temperatur sowie Polymer- und Monomer-Konzentration nahe denen bei der Polymersynthese liegen. Durch die Verwendung eines Inhibitors (DtBMP) und durch den Ausschluss von Initiatoren und initiierenden Verunreinigungen können chemische Umsetzungen verhindert werden.

Es wurden alle im Rahmen dieser Arbeit aufgenommenen Trübungsdruckkurven, um die chemische Reaktion begünstigende, thermische Belastung der Proben möglichst gering zu halten, bei aufsteigender Temperatur gemessen. Zum Ende jeder Messung wurden stichprobenartig mehrere Bedingungen bei absteigender Temperatur erneut gemessen. Dabei konnten die Trübungsdruckdaten innerhalb des jeweiligen Experiments bestätigt werden.

Zur Kontrolle der grundsätzlichen Reproduzierbarkeit der Trübungsdruckmessungen wurden ausgewählte Polymerproben in unabhängigen Experimenten wiederholt vermessen. In Abbildung 5.4 sind die Ergebnisse am Beispiel der Trübungsdruckkurven von Polypropylacrylat und einem Ethen-Ethylmetharylat-Copolymer $\left(\mathrm{E}_{81.0} \mathrm{EMA}_{19.0}\right)$ illustriert. Die für das jeweilige System an verschiedenen Tagen erhaltenen Trübungsdrücke lassen sich 
unter Berücksichtigung der Messgenauigkeit (vgl. Abschnitt 3.4) nicht unterscheiden. Mit aufgeführt in Abbildung 5.4 sind die Ergebnisse zweier Messungen an einem E-BzMACopolymer $\left(\mathrm{E}_{96.7} \mathrm{BzMA}_{3.3}\right)$. Für diese Probe wurde, um die Langzeitstabilität der EthenCopolymer-Mischungen zu kontrollieren, die Mischung nach Aufnahme der ersten Messreihe 5 Stunden unter Messbedingungen (1500 bar, $\left.240{ }^{\circ} \mathrm{C}\right)$ belassen, bevor eine weitere Messreihe durchgeführt wurde.

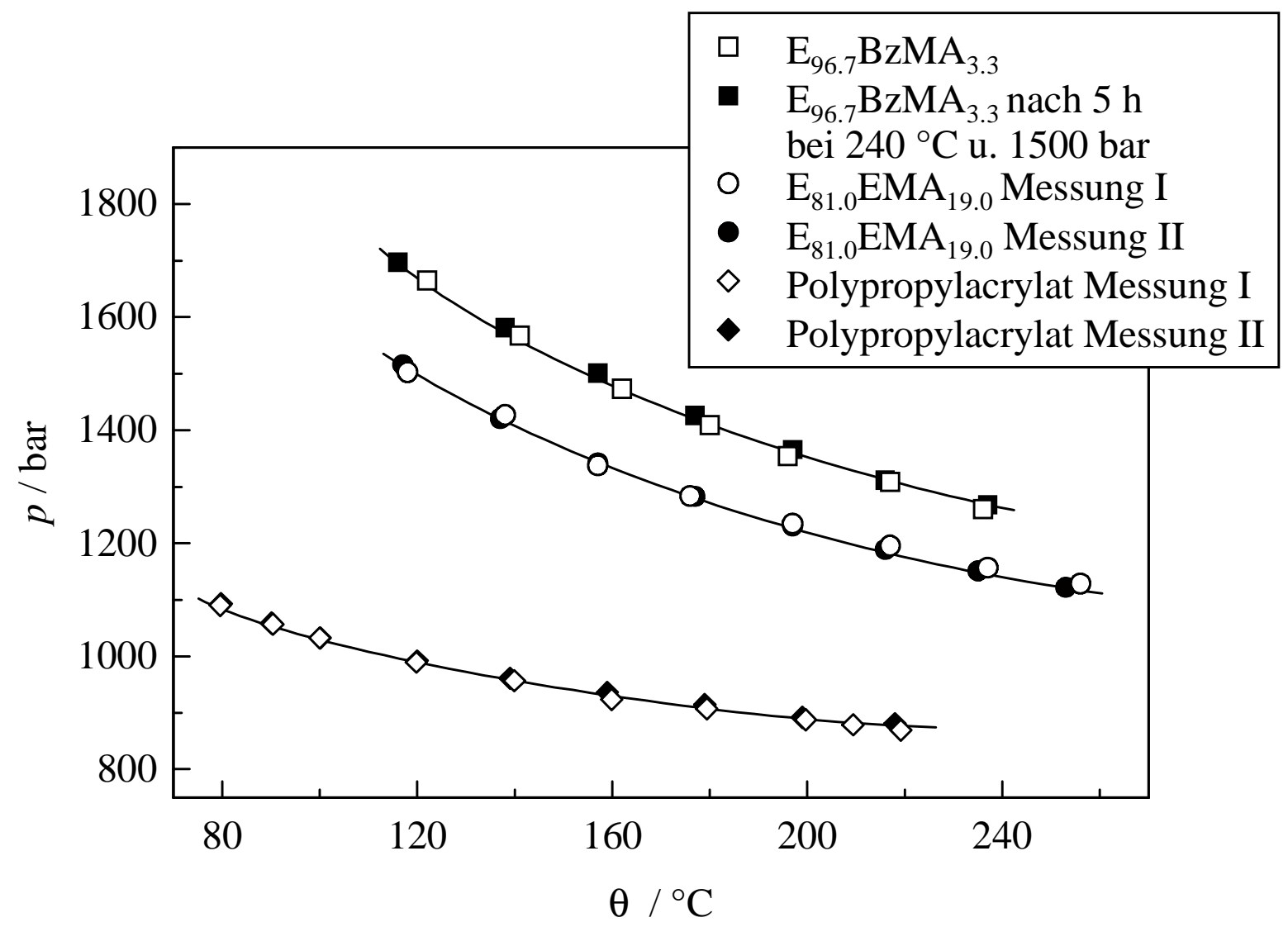

Abbildung 5.4: Trübungsdruckkurven von $E_{96.7} B z M A_{3.33}$ vor und nach 5 h Lagerung unter Messbedingungen. Ergebnisse wiederholt ausgeführter Trübungsdruckmessungen an Ethen-Polypropylacrylat und Ethen-E-EMA-Mischungen.

Die Dauer des Experiments wurde somit mehr als verdoppelt. Die Gesamtmesszeit betrug 9 h. Die Copolymere werden so einer wesentlich höheren thermischen Belastung ausgesetzt als während eines Standardexperiments. Die offensichtlich sehr gute Übereinstimmung der Trübungsdrücke auch nach mehrstündiger Lagerung der potentiell reaktiven Mischung macht die hervorragende Reproduzierbarkeit der verwandten Messmethode deutlich.

Reproduzierbare Ergebnisse schließen jedoch nicht grundsätzlich aus, dass dennoch chemische Reaktionen während der Messungen ablaufen. So ist denkbar, dass eventuelle Umsetzungen bei jedem Experiment in gleichem Ausmaß stattfinden oder auch, dass diese Reaktionen den Trübungsdruck nicht signifikant beeinflussen. Ein möglicher Vorgang ist 
zum einen ein Aufpolymerisieren von Ethen auf das Copolymer zum anderen das Entstehen von Polyethylen. Weiterhin können eventuell ablaufende Depropagationsreaktionen die Messungen beeinflussen.

In der Literatur finden sich Hinweise, dass die Stabilisierung von Ethen-CopolymerSystemen problematisch ist. So berichtet Müller [9] von Polymerisationen bei Trübungsdruckmessungen an Mischungen aus Ethen und Ethen-Methylacrylat-Copolymeren. Die Menge des während der Experimente entstandenen Polyethylens erwies sich als abhängig von der Reinheit des verwendeten Ethens und von Inhibitortyp und -menge [10]. Es gelang dem Autor jedoch nicht, die Polymerisation vollständig zu unterdrücken. Wind [11] beobachtete eine zunehmende Rot/Braunverfärbung der von ihm untersuchten Ethen / Ethen-Acrylsäure-Copolymer-Mischungen bei hohen Temperaturen und ein Abfallen der Trübungsdrücke nach sehr langen Standzeiten (48 Stunden bei $200{ }^{\circ} \mathrm{C}$ ). Dies führt Wind auf Zersetzungsreaktionen zurück. In der vorliegenden Arbeit wurde daher besondere Sorgfalt darauf verwandt, sicherzustellen, dass die ausgeführten Trübungspunktmessungen rein thermodynamische Experimente an durchweg stabilen Mischungen sind. Hierzu wurde die Mehrzahl der Polymere vor und nach jedem Experiment untersucht. Mögliche Depropagationsreaktionen sollten zu niedrigeren Molmassen, das Aufpolymerisieren von Polyethylen zu einem geringerem (Meth)Acrylatgehalt der Proben führen. Werden beide Größen überprüft, lassen sich alle eventuellen Kombinationen unerwünschter Nebenreaktionen feststellen. Selbst das Aufpolymerisieren von Polyethylen bei gleichzeitiger Depropagation unter Erhalt der Molmassenverteilung würde durch die veränderte Zusammensetzung detektierbar. Entsprechend kann die Inhibierung bei einer Trübungsdruckmessung nur dann als gelungen betrachtet werden, wenn Molekulargewichtsverteilung und Copolymerzusammensetzung während des Experiments unverändert bleiben.

In Abbildung 5.5 werden exemplarisch Molekulargewichtsverteilungen von Proben der Copolymersysteme E-EA, E-PA, E-MMA und E-EMA gezeigt. Dargestellt sind die Verteilungen der Proben jeweils vor und nach einem Trübungspunktexperiment. 


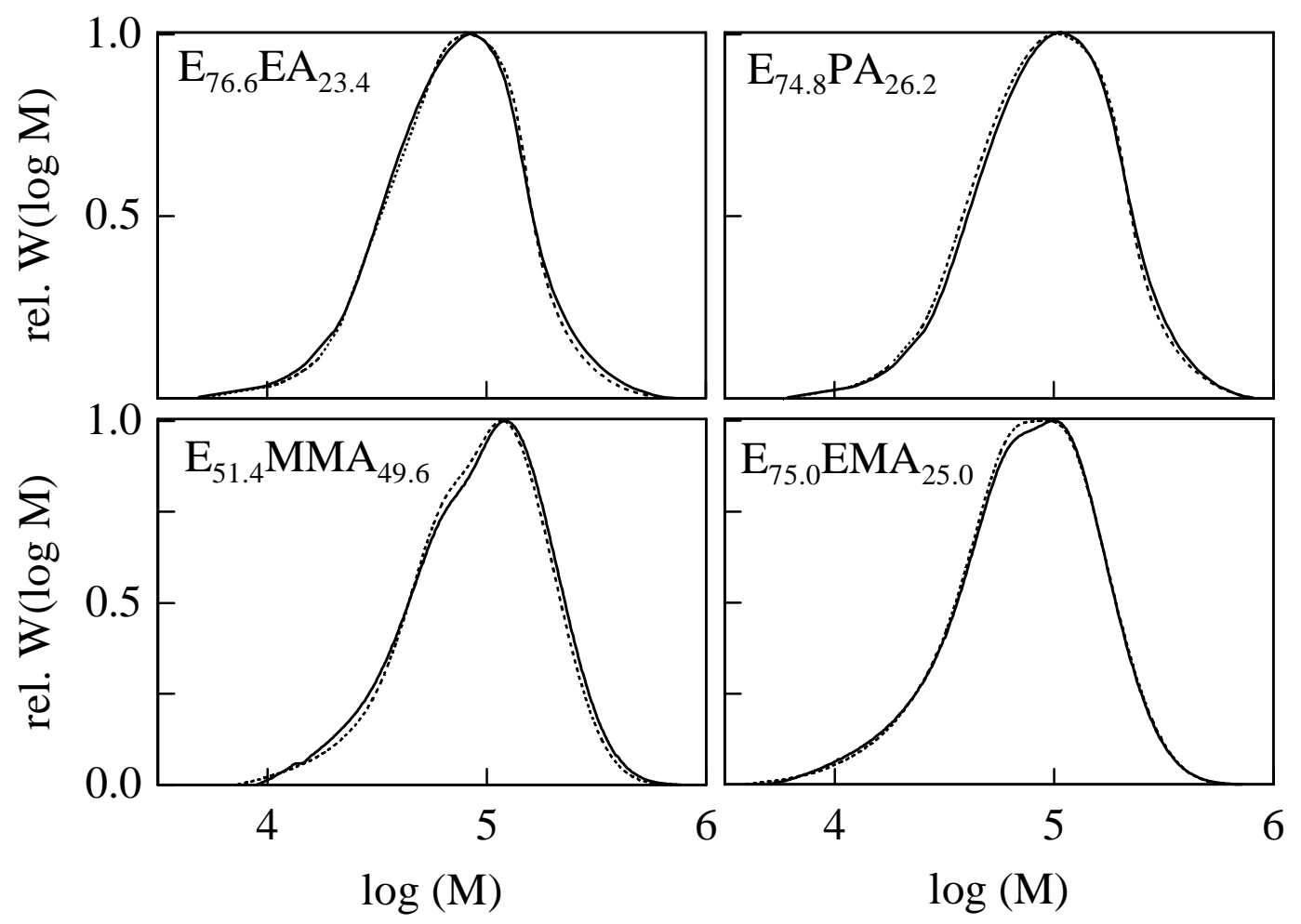

Abbildung 5.5: Molmassenverteilung verschiedener Ethen-Copolymere vor und nach Trübungsdruckmessungen (durchgezogene bzw. unterbrochene Linien).

Für alle Copolymersysteme wird eine sehr gute Übereinstimmung der beiden jeweils erhaltenen Verteilungen deutlich. Es werden keine Hinweise auf Polymerisations- bzw. Depropagationsvorgänge während der Trübungspunktexperimente gefunden.

Die (Meth)Acrylatgehalte der E-MMA-Copolymere wurden mit der in Abschnitt 3.2.4 vorgestellten IR / ${ }^{1} \mathrm{H}$-NMR-Korrelation untersucht. Dazu wurden von den Copolymeren vor und nach den Trübungsdruckmessungen NIR/IR-Filmspektren aufgenommmen und diese miteinander verglichen. Abbildung 5.6 zeigt beispielhaft zwei IR-Spektren von $\mathrm{E}_{74.1} \mathrm{MMA}_{25.9}$-Proben, wobei nur eine zuvor dem Trübungspunktexperiment unterworfen worden war (Spektrum mit unterbrochener Linie dargestellt). Die Intensitäten im zur Gehaltsbestimmung ausgewerteten Carbonyl- und CH-Bereich (um 3450 bzw. $2500 \mathrm{~cm}^{-1}$ ) sind identisch und ergeben unter Verwendung der Korrelation gleiche Methacrylatgehalte (siehe Abschnitt 3.2.4).

Auffällig ist allerdings die im post experimentellen Spektrum auftretende Absorbanz zwischen 3500 und $3700 \mathrm{~cm}^{-1}$, welche im Spektrum der Ausgangsprobe nicht vorhanden ist. Diese bei allen untersuchten Copolymeren auftretende Absorbanz ist nicht auf chemische Veränderungen, sondern auf den Inhibitor (DtBMP) zurückzuführen, der nach den Trübungsdruckexperimenten in den Proben verbleibt. 


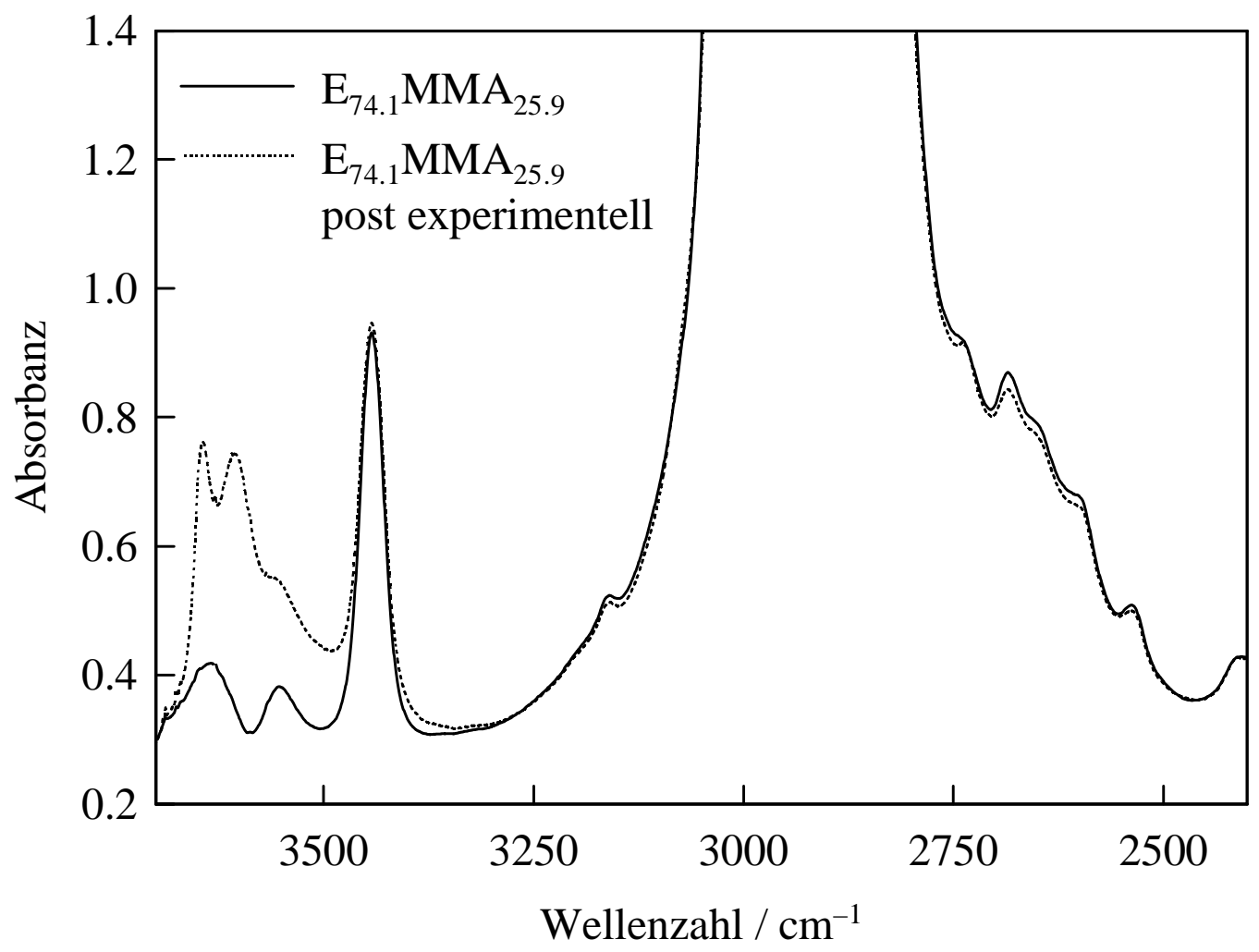

Abbildung 5.6: IR/NIR-Absorptionsspektren eines Polymeren vor und nach einem Trübungspunktexperiment.

Um dies zu belegen wurden Proben eines Copolymers mit unterschiedlichem Inhibitorgehalt hergestellt. Hierzu wurde Copolymer und eine entsprechende Menge Inhibitor in Tetrahydrofuran gelöst und anschließend das Lösungsmittel unter vermindertem Druck entfernt. In Abbildung 5.7 sind IR/NIR-Absorptionsspektren von zwei dieser Proben sowie die der entsprechenden Ausgangs- und post-experimentellen Probe dargestellt. Die Zunahme der fraglichen Bande mit steigendem Gehalt an physikalisch gelöstem Inhibitor ist klar erkennbar. Diese Bande ist OH-Streckschwingungen zuzuordnen, welche bekanntermaßen hohe Molarabsorptivitäten aufweisen. Die Intensität im wenig strukturierten CH-Schwingungsbereich des Polymers ändert sich hingegen durch die $\mathrm{CH}-$ Gruppen des Inhibitormoleküls nicht merklich.

Es sei angemerkt, dass die Intensität der Banden um $3600 \mathrm{~cm}^{-1}$ für die post-experimentelle und die Probe mit 10 Gew.\% Inhibitor fast identisch ist, weil dies nahezu der tatsächlichen Einwaage an Polymer und Inhibitor entspricht (siehe Abschnitt 4.3.3). Dies zeigt weiterhin, dass durch das Spülen mit Ethen der mit Polymer und Inhibitor beschickten Zelle vor dem Experiment praktisch kein Inhibitor aus der Messzelle ausgetragen wird. 


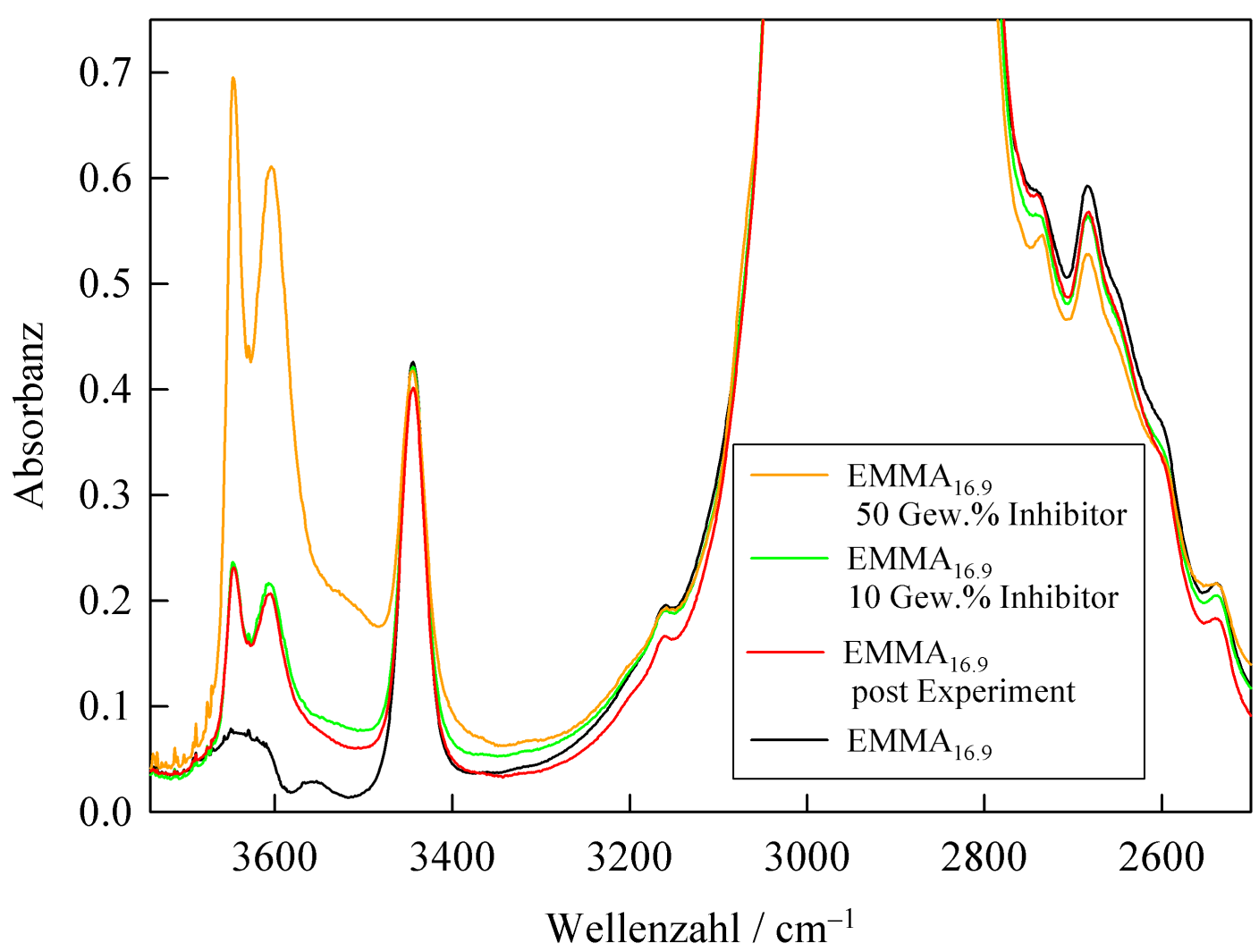

Abbildung 5.7: IR/NIR-Absorptionsspektren von $E_{83.1} M M A_{16.9}$ bei Anwesenheit unterschiedlicher Inhibitorkonzentrationen

Zusätzlich zu den vorgestellten Polymer-Charakterisierungen wurden stichprobenartig auch Elementaranalyse und ${ }^{1} \mathrm{H}-\mathrm{NMR}-$ Spektroskopie zur Analyse von Proben vor und nach dem thermodynamischen Experiment eingesetzt. Auch diese Techniken zeigten keine Hinweise auf Veränderungen an den Polymerproben.

Zusammenfassend kann festgehalten werden, dass anhand der unter Anwendung verschiedener Analysetechniken ausgeführten, sorgfältigen Untersuchungen der Polymere vor und nach den Trübungsdruckexperimenten keine chemischen Veränderungen durch die Trübungsdruckmessung feststellbar waren. Es kann daher davon ausgegangen werden, dass die im Rahmen dieser Arbeit untersuchten Ethen-Polymer-Mischungen durch den eingesetzten Inhibitor ausreichend stabilisiert werden konnten.

\subsubsection{Einfluss durch Verwendung eines Inhibitors}

Grundsätzlich muss berücksichtigt werden, dass die Anwesenheit des eingesetzten Inhibitors DtBMP (Anteil ca. 0.5 Gew.\%) einen Einfluss auf das Entmischungsverhalten der untersuchten Systeme haben kann. Aufgrund der Polarität des DtBMP-Moleküls ist eine löslichkeitsverbessernde Wirkung denkbar. Ergebnisse von Trübungspunktmessungen von 
Dietzsch [12] am System Ethen / Ethen-Butylacrylat-Copolymer zeigen jedoch, dass eine Variation der eingesetzten Inhibitormenge zwischen 0.011-1.486 Gew.\% D $t$ BMP im Rahmen der Unsicherheit der Trübungsdruckmessung keinen Einfluss hat. Es kann angenommen werden, dass dies auch für die in dieser Arbeit untersuchten eng verwandten Ethen / Ethen-(Meth)Acrylat-Copolymer-Systeme gilt.

\subsubsection{Einfluss der Polymerkonzentration}

Für viele Solvens-Homopolymer-Systeme sind Daten zur Abhängigkeit des Entmischungsdrucks von der Polymerkonzentration bekannt. Aufgrund der großen wirtschaftlichen Bedeutung ist das System Ethen-Polyethylen besonders gut untersucht [z.B. 13]. Im Bereich der im Hochdruckverfahren hergestellten, ethenbasierten Copolymere existieren systematische Studien bisher nur für Mischungen aus Ethen und Ethen-Methylacrylat-Copolymeren [9] bzw. Ethen-Acrylsäure-Copolymeren (E-AS) [11]. Es hat sich gezeigt, dass der formale Verlauf der Trübungsdrücke mit der Polymerkonzentration für die Ethen / Copolymer- und Ethen / Polyethylen-Systeme sehr ähnlich ist. Die Drücke steigen mit zunehmender Polymer-konzentration zunächst an. Im Bereich eines Polymergewichtsbruchs von etwa 2 $7 \%$ wird ein flaches Maximum gefunden. Zu höheren Polymerkonzentrationen fällt der Trübungs-druck stetig ab. In Übereinstimmung mit den in [9] und [11] für E-MA- und EAS-Copolymere gefundenen Maxima haben Experimente mit variierender Polymerkonzentration im Bereich von 1.0 bis $6.3 \mathrm{Gew} \%$ (Plateaubereich) für ein Ethen-ButylacrylatCopolymer innerhalb der Messgenauigkeit identische Trübungsdrücke ergeben [12]. Es kann angenommen, dass dies auch für die hier untersuchten Ethen-(Meth)-Acrylsäure(ester)-Copolymere gilt.

Alle im Rahmen dieser Arbeit in der diskontinuierlichen Apparatur untersuchten Copolymere wurden bei einem Polymergewichtsbruch von ca. $5 \%$ gemessen. Der Verlust an Ethen, der durch das Spülen der Zuleitung entsteht, sowie die Schwankungen der Ethenmenge, die durch die Methode der Eindosierung auftreten, führen zu einer maximalen Abweichung des Polymergewichtbruchs von $\pm 0.2 \%$. Aufgrund der vorangehend genannten Ergebnisse sollte dies keinen Einfluss auf die Lage der Trübungsdrücke haben.

Zur Übertragung der in dieser Arbeit erhaltenen Trübungsdruckdaten auf die industrielle Anwendung ist zu beachten, dass in der technischen Reaktionsmischung in Abhängigkeit von der gewählten Copolymerzusammensetzung und dem Umsatz verschieden große Mengen an noch nicht umgesetztem Comonomer vorhanden sind, welche eine mehr oder 
weniger große Cosolvenswirkung [12] haben. Darüber hinaus ist davon auszugehen, dass in der Praxis üblicherweise Umsätze zwischen 15 - 35\% gefordert sind und somit erheblich höhere Copolymerkonzentrationen erreicht werden. Die in dieser Arbeit für binäre Systeme bei Polymerkonzentrationen von 5 Gew.\% bestimmten Trübungsdrücke ent-sprechen daher den höchstmöglichen für diese Systeme zu beobachtenden Trübungsdrücken (s.o.) und gelten somit für den "worst case".

\subsubsection{Reproduzierbarkeit der Ergebnisse der Trübungsdruckmessungen mit der kontinuierlichen Apparatur}

Die grundsätzliche Reproduzierbarkeit der mit der in Abschnitt 3.3.4 erläuterten „,inline“Technik gewonnenen Ergebnisse konnte durch unabhängige Experimente an verschiedenen Versuchstagen belegt werden.

Einer der Vorteile der kontinuierlichen Trübungsdruckmessung besteht darin, dass die untersuchten Copolymere im Vergleich zur Satzmethode nur kurzzeitig thermischer Belastung ausgesetzt sind. Dieser Zeitraum entspricht gerade der Verweilzeit von etwa $8 \mathrm{~s}$ in der zuführenden Kapillare und in der dem Synthesereaktor nachgeschalteten Hochdruckzelle. Dennoch ist es notwendig zu kontrollieren, ob die Polymere in dieser Zone weiteren Reaktionen ausgesetzt sind. Da die Copolymerisation von Ethen und (Meth)Acrylsäureestern stark temperaturabhängig ist, sollten sich für den Fall von Reaktionen in der nachgeschalteten Zelle bei verschiedenen Temperaturen Änderungen von Umsatz und eventuell auch von Zusammensetzung und Molekulargewichtsverteilung der Polymere ergeben. Daher wurden Copolymerisationsexperimente bei einer Synthesetemperatur von $200{ }^{\circ} \mathrm{C}$ durchgeführt, wobei die nachgeschaltete Hochdruckzelle zunächst auf 100 und später auf $240{ }^{\circ} \mathrm{C}$ geheizt wurde. Es konnte dabei keine Umsatzänderung festgestellt werden. Die synthetisierten Copolymere wurden mittels ${ }^{1} \mathrm{H}-\mathrm{NMR}-$ Spektroskopie und GPC analysiert. Trotz der sehr unterschiedlichen Temperaturen in der Trübungsdruckmesszelle wiesen die Methacrylatgehalte und Molekulargewichtsverteilungen der Proben keine Unterschiede auf. Es ist daher davon auszugehen, dass keine oder zumindest keine signifikante Veränderungen der Copolymere durch diese Messtechnik eintreten. 


\subsection{Ergebnisse der Trübungsdruckmessungen an Mischungen aus Ethen und Ethen-(Meth)Acrylat-Copolymeren}

Im folgenden Abschnitt werden die Ergebnisse der Trübungspunktmessungen an Mischungen aus Ethen und Ethen-(Meth)Acrylat-Copolymeren vorgestellt.

Alle Messungen wurden bei einem Polymergewichtsbruch von ca. 5\% und unter Anwendung von ca. 0.5 Gew.\% DtBMP als Stabilisator ausgeführt. Eine Ausnahme sind die Experimente zu den ternären Systemen (s. Abschnitt 5.5) bei denen kein Inhibitor notwendig ist und der Reaktionsumsatz die Polymerkonzentration bestimmt. Im Anhang B sind die Zusammensetzung und die mittleren Molmassen der vermessenen Proben angegeben. Eine Auflistung der Messdaten der Trübungspunktexperimente findet sich im Anhang C.

\subsubsection{System Ethen / Ethen-Ethylacrylat-Copolymer}

In Abbildung 5.8 sind die für Mischungen aus Ethen und Ethen-Ethylacrylat-Copolymeren mit verschiedenem Ethylacrylatgehalt erhaltenen Trübungsdrücke in Abhängigkeit von der Temperatur dargestellt. Neben den für das Randsystem Ethen/Poly(EA) bestimmten Kurven ist zu Vergleichszwecken eine Trübungskurve für das System Ethen / Polyethylen eingezeichnet. Zum besseren Verständnis sei erwähnt, dass die untersuchten Mischungen unterhalb der Trübungskurven zweiphasig und oberhalb homogen vorliegen.

Die Trübungsdrücke aller untersuchten Mischungen fallen mit zunehmender Temperatur mit ähnlicher Charakteristik ab. Weiterhin ist erkennbar, dass die Kurven nahezu unabhängig vom Comonomergehalt mit ansteigender Temperatur weniger stark abfallen und zunehmend parallel verlaufen. Die Steigungen der einzelnen Trübungsdruckkurven sind oberhalb von etwa $200{ }^{\circ} \mathrm{C}$ praktisch ununterscheidbar. Zur Illustration des Einflusses der Copolymerzusammensetzung auf das Phasenverhalten ist in Abbildung 5.8, 5.9 und 5.11 - 5.13 ein Pfeil eingefügt, welcher jeweils die Abfolge der Trübungsdruckkurven mit zunehmendem (Meth)Acrylatgehalt angibt. Der Trübungsdruck nimmt ausgehend vom Polyethylen bis zur Probe mit einem EA-Gehalt von 28.4 mol-\% zunächst ab. Bei weiterer Zunahme des Comonomergehalts steigt der Trübungsdruck bis zum entsprechenden Randsystem Ethen/Polyethylacrylat geringfügig wieder an, wobei diese Mischung im gesamten Temperaturbereich niedrigere Trübungsdrücke aufweist als das (zweite) Randsystem Ethen / Polyethylen. 


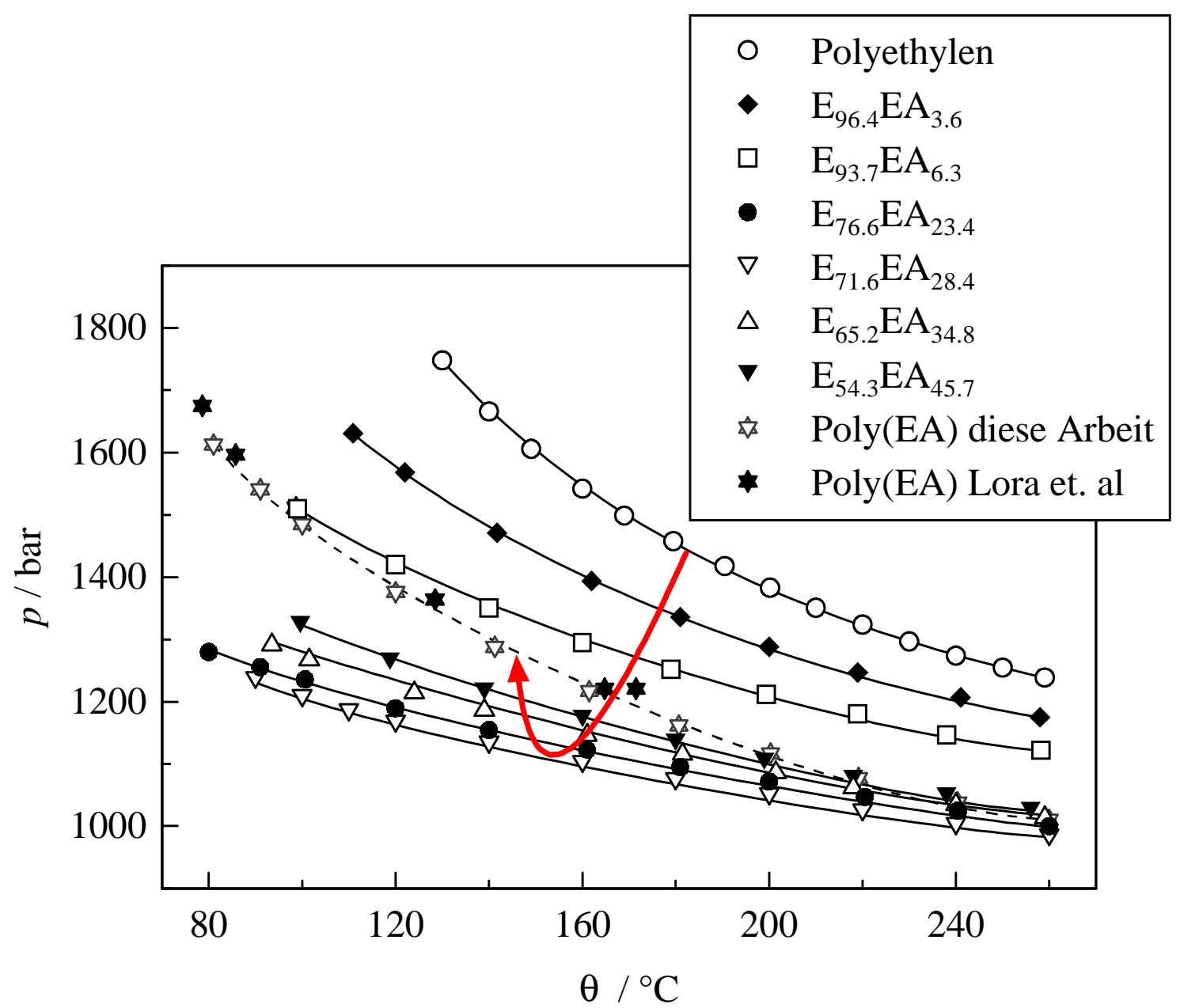

Abbildung 5.8: Trübungsdruckkurven für Mischungen aus Ethen und Ethen-EthylacrylatCopolymeren mit verschiedenen Ethylacrylatgehalten sowie für Mischungen aus Ethen mit Polyethylen bzw. Polyethylacrylat.

Die hervorragende Übereinstimmung der Ergebnisse dieser Arbeit für Ethen / Pol(EA) (offene Sternsymbole) mit Literaturergebnissen von Lora et al. [14] (gefüllte Sternsymbole) demonstriert nochmals die gute Reproduzierbarkeit der verwendeten experimentellen Technik.

Die zunehmende Steigung der Trübungsdruckkurven zu niedrigeren Temperaturen beruht auf intrasegmentellen Dipol-Dipol-Wechselwirkungen der Acrylat-Einheiten, welche die Löslichkeit verschlechtern. Diese sind umgekehrt proportional zur Temperatur und gewinnen so mit abnehmender Temperatur an Bedeutung. Mit zunehmender Temperatur dominieren die temperaturunabhängigen Dispersionswechselwirkungen und der Effekt der polaren Carboxylatgruppen innerhalb der Acrylatbausteine nimmt ab, bis letzlich die Trübungsdruckkurven der verschiedenen Copolymere nahezu identische Steigungen aufweisen. 


\subsubsection{System Ethen / Ethen-Propylacrylat-Copolymer}

In Abbildung 5.9 werden die Ergebnisse der Messungen an Mischungen aus Ethen und Ethen-Propylacrylat-Copolymeren gezeigt. Die Verlaufscharakteristik der Trübungsdruckkurven ist derjenigen für das E-EA-Copolymersystem sehr ähnlich. Während für das System Ethen / Ethen-Ethylacrylat-Copolymer mit zunehmendem Acrylat-Gehalt ein flaches Minimum des Trübungsdrucks beobachtet wird, sinken die Trübungsdrücke der Mischungen aus Ethen und Ethen-Propylacrylat-Copolymer mit dem Propylacrylatgehalt im Copolymer bis zum Randsystem Ethen / Polypropylacrylat stetig.

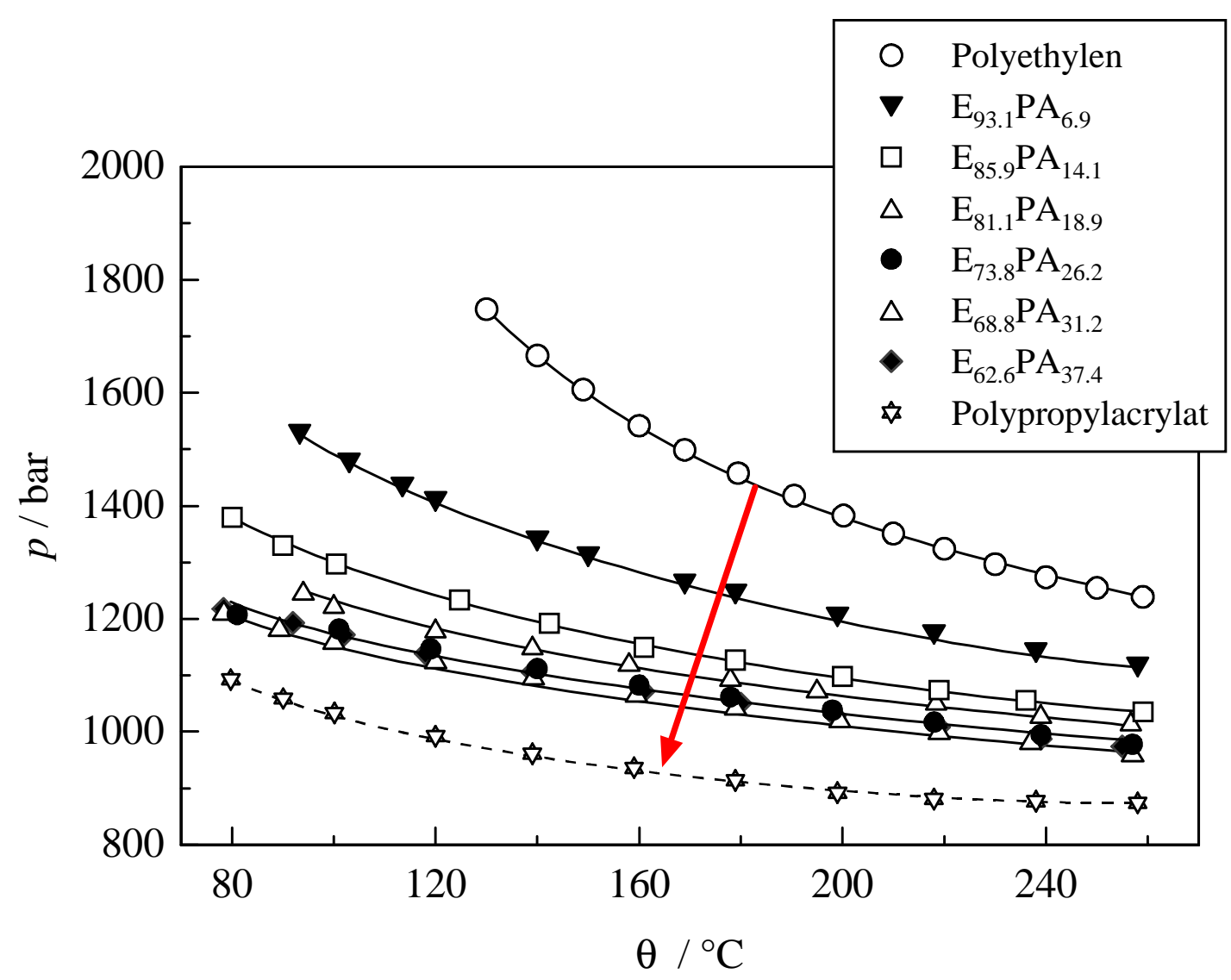

Abbildung 5.9: Trübungsdruckkurven für Mischungen aus Ethen und Ethen-Propylacrylat-Copolymeren mit verschiedenen Propylacrylatgehalten sowie für Mischungen aus Ethen mit Polyethylen bzw. mit Polypropylacrylat.

In Abbildung 5.10 werden in einer Auftragung der Trübungsdrücke bei $200^{\circ} \mathrm{C}$ gegen die Copolymerzusammensetzung $\left(F_{\mathrm{A}}\right)$ die Daten der bislang untersuchten Systeme vom Typ Ethen / Ethen-Acrylat-Copolymer verglichen. Für das E-MA-System werden die Daten von Buyn et al. [15] und Ergebnisse aus Ref. [16] dargestellt. Die Trübungsdruckdaten für das Ethen / E-BA-System entstammen [15] und [12], wobei in der vorliegenden Arbeit eine ergänzende Messung ausgeführt wurde (Datenpunkt für $F_{\mathrm{A}}=26.8 \mathrm{~mol}-\%$ ). Die Trübungs- 
drücke der erstmals vermessenen Systeme E / E-EA und E / E-PA fügen sich gut zwischen die beiden bereits untersuchten Systeme E / E-MA und E / E-BA ein. Die Trübungsdrücke der Systeme E / E-EA, E / E-PA und E / E-BA sind bei Copolymer-Acrylatgehalten unter 20 mol-\% im Rahmen der Messgenauigkeit nicht unterscheidbar. Zu höheren Acrylatgehalten hin liegt der Trübungsdruck umso niedriger, je größer der Alkyl-Esterrest ist. Allerdings sind die absoluten Unterschiede im Trübungsdruck mit weniger als 80 bar nur gering.

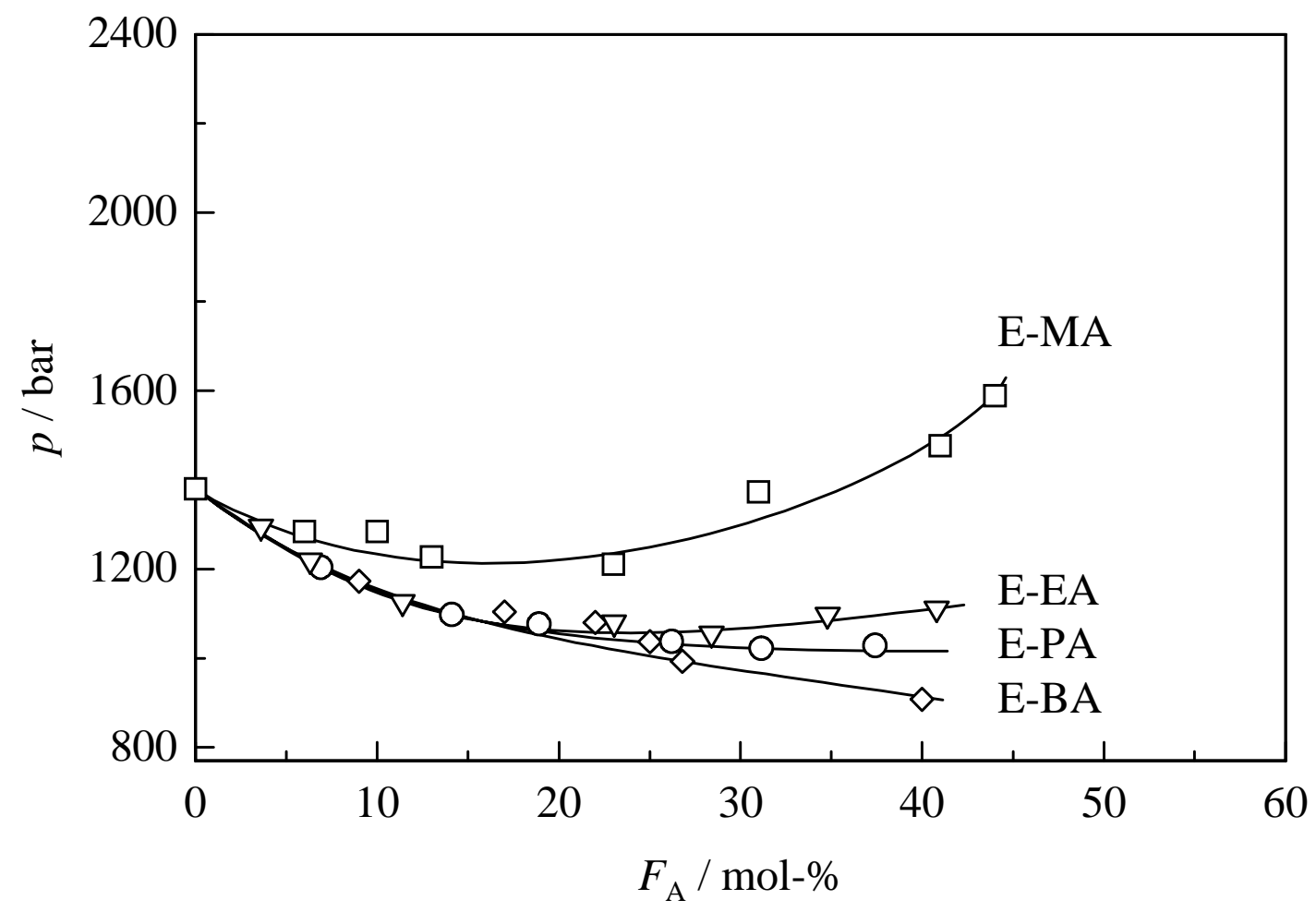

Abbildung 5.10: Einfluss der Copolymerzusammensetzung auf den Trübungsdruck für Ethen-Acrylat-Copolymere bei $200^{\circ} \mathrm{C}$ in Ethen. Die Daten für E-MA sind [15] und [16] entnommen. Die E-BA-Daten entstammen [15], [12] und dieser Arbeit.

Die unterschiedlichen Trends im Entmischungsverhalten der Copolymere lassen sich durch verschieden starke Wechselwirkungen polarer Segmente innerhalb eines Copolymermoleküls verstehen. Die die Löslichkeit ungünstig beeinflussenden Dipol-Dipol-Wechselwirkungen zwischen den Acrylatbausteinen wird umso stärker gestört, je größer die Alkylgruppe des Esters und damit die Abschirmung der polaren Carboxylatgruppe der Acrylate ist. Darüberhinaus erhöhen größere Alkylgruppe an der Esterfunktion die Oberfläche der Copolymerketten, so dass verstärkt effektive, die Löslichkeit verbessernde PolymerSolvens-(Dispersions)-Wechselwirkungen ausgebildet werden können. Im Vergleich zum E-MA-System wird anhand der Daten für E-EA und E-PA deutlich, dass sich die 
Charakteristik des Entmischungsverhaltens der Ethen / Ethen-Copolymer-Systeme mit der Größe des Alkyl-Esterrest nicht stetig ändert. Das System E-MA nimmt eine Sonderstellung ein. Die Trübungsdrücke für E-MA sind nahezu im gesamten untersuchten Zusammensetzungsbereich erheblich höher als die der Systeme mit größerer Alkylestergruppe. Darüber hinaus wird nur für das System E-MA ein ausgeprägtes Minimum für die Abhängigkeit des Trübungsdrucks vom Acrylatgehalt im Copolymer gefunden. Gleichzeitig ist das zugehörige Homopolymer Polymethylacrylat im Vergleich zu den anderen Polyacrylaten das einzige, welches im Zustandsbereich bis 3000 bar und $260^{\circ} \mathrm{C}$ nicht mit Ethen homogen mischbar ist. Dies zeigt, dass das Anfügen der ersten $\mathrm{CH}_{2}$-Gruppe (d.h. der Übergang von E-MA zu E-EA) offensichtlich die Abschirmung der Carboxylgruppe entscheidend beeinflusst. Eine weitere Verlängerung dieses Esterrests führt nur zu geringfügiger Verbesserung der Löslichkeit.

\subsubsection{System Ethen / Ethen-Methylmethacrylat-Copolymer}

In Abbildung 5.11 sind Trübungsdrücke für Mischungen aus Ethen und E-MMACopolymeren mit verschiedenem Methylmethacrylatgehalt in Abhängigkeit von der Temperatur dargestellt. Eine für das System Ethen / Polyethylen bestimmte Trübungspunktkurve dient wieder zum Vergleich.

Der Verlauf der Trübungsdruckkurven ähnelt dem für Ethen-Acrylat-Copolymere. Anfänglich wird mit zunehmenden MMA-Gehalt ein Absinken der Trübungsdrücke festgestellt. Die geringsten Drücke werden für ein Copolymer mit einem MMA-Gehalt von 18.5 mol-\% gemessen. Vergleichbar mit dem homologen System E-MA steigt der Trübungs-druck mit weiter zunehmenden MMA-Gehalt der Copolymere erheblich an, so dass eine E-MMA-Probe mit nahezu 50 mol-\% MMA höhere Trübungsdrücke als Polyethylen aufweist. Messungen an E-MMA-Copolymeren mit noch höherem MMAGehalt zeigen, dass der Anstieg des Drucks mit dem Gehalt oberhalb von ca. 50 mol-\% sehr steil ist. So erwies sich eine Probe mit 54 mol-\% bei Temperaturen um $240^{\circ} \mathrm{C}$ innerhalb des zulässigen Maximal-drucks als gerade noch löslich. Proben von noch höherem Gehalt und das zugehörige Randsystem Ethen/Polymethylmethacrylat konnte (vgl. Abschnitt 6.4) innerhalb der apparativen Grenzen nicht in Lösung gebracht werden. 


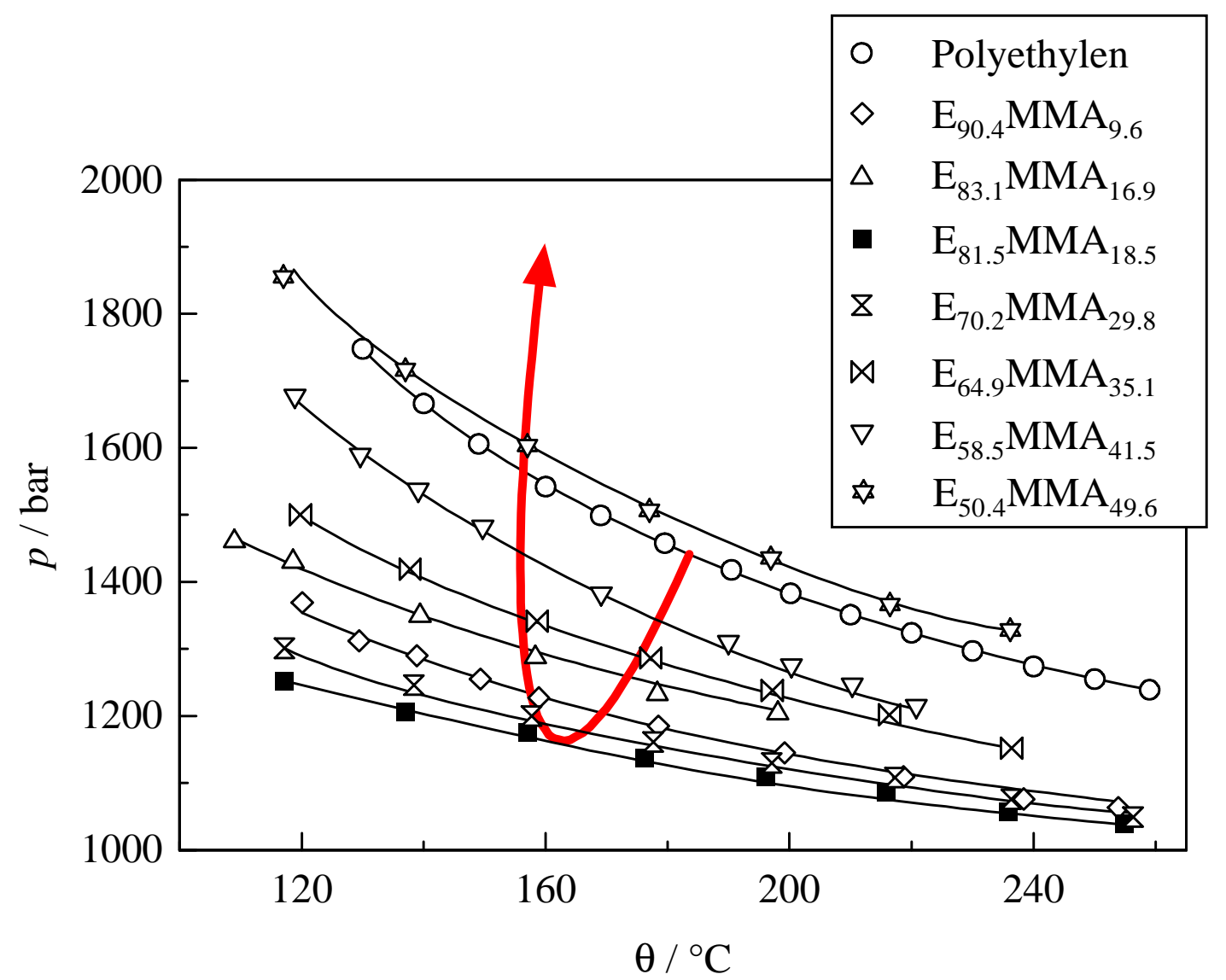

Abbildung 5.11: Trübungsdruckkurven für Mischungen aus Ethen und Ethen-Methylmethacrylat-Copolymeren mit verschiedenen Estergehalten

\subsubsection{System Ethen / Ethen-Butylmethacrylat-Copolymer}

In Abbildung 5.12 sind die Trübungsdruckkurven für Mischungen aus Ethen und EthenButylmethacrylat-Copolymeren mit verschiedenem Comonomergehalt und die Ergebnisse für das entsprechende Homopolymer Polybutylmethacrylat dargestellt. Ausgehend von Polyethylen nehmen die Trübungsdrücke mit zunehmendem Butylmethacrylatgehalt bis zu einen BMA-Gehalt von 33.8 mol-\% stetig ab. Interessanterweise steigen im Unterschied zum homologen System Ethen/E-BA-Copolymer die Trübungsdrücke von Ethen/EBMA-Copolymer mit weiter zunehmendem Methacrylatgehalt der Proben in Richtung des Randsystem Ethen / Polybutylmethacrylat wieder an. Das Auftreten eines Trübungsdruckminimums wird anhand des in die Abbildung eingefügten Pfeils verdeutlicht. 


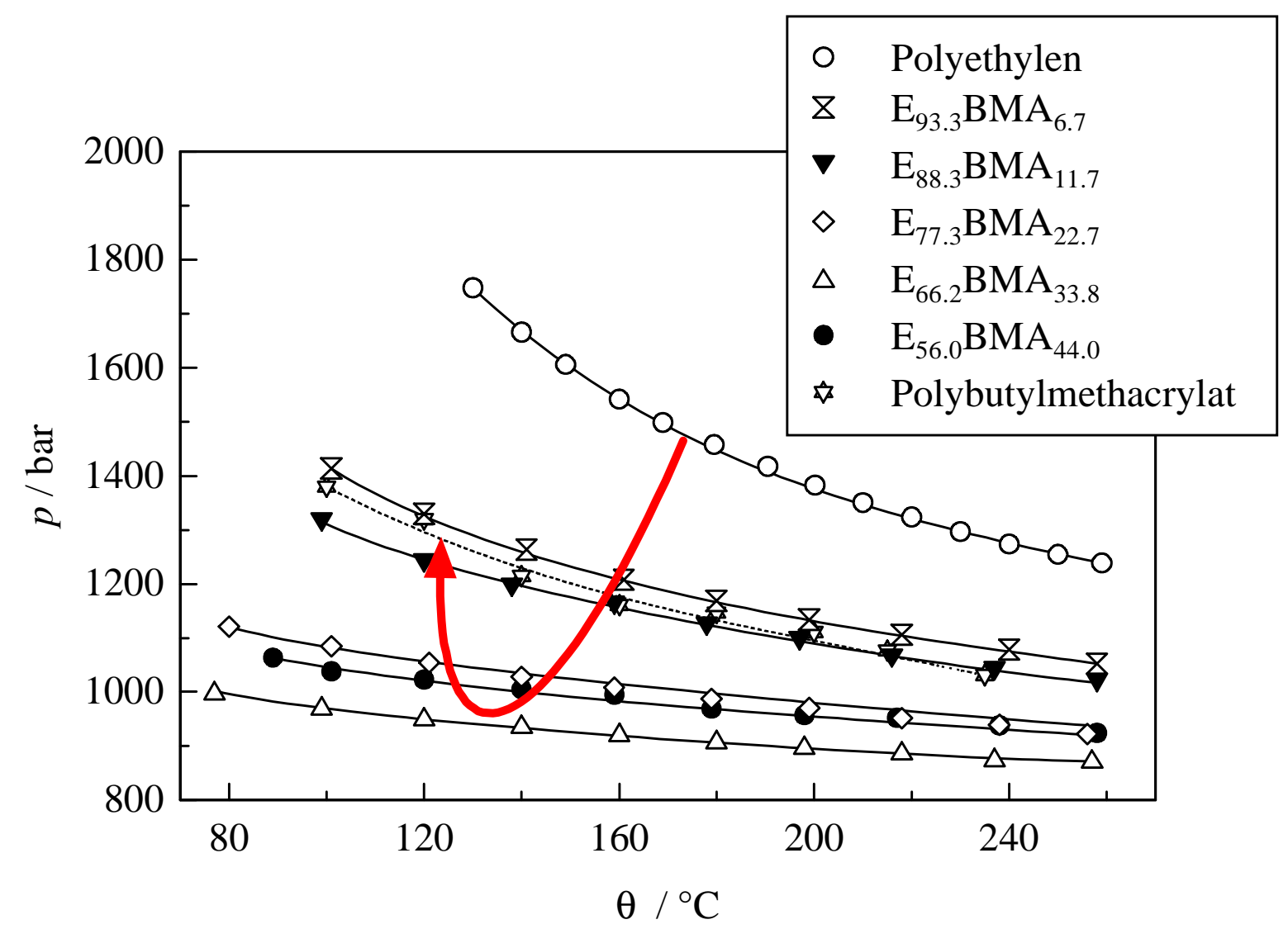

Abbildung 5.12: Trübungsdruckkurven für Mischungen aus Ethen und Ethen-Butylmethacrylat-Copolymeren mit verschiedenen BMA-Gehalten, sowie für Mischungen aus Ethen mit Polyethylen bzw. Polybutylmethacrylat.

\subsubsection{System Ethen / Ethen-Benzylmethacrylat-Copolymer}

In dieser Arbeit wurden erstmalig Trübungsdrücke für ein Ethen-(Meth)Acrylat-CopolymerSystem mit einem funktionalisierten (Meth)Acrylat-Baustein gemessen. Das System EthenBenzylmethacrylat erschien interessant, weil E-BzMA ein Beispiel für ein Ethen-Copolymer mit besonders sperriger Alkylestergruppe darstellt. So wäre zu erwarten, dass E-BzMACopolymere aufgrund der großen Seitengruppen besonders niedrige Trübungsdrücke aufweisen. In Abbildung 5.13 werden die für Mischungen aus Ethen und E-BzMACopolymeren mit unterschiedlicher Zusammensetzung bestimmten Trübungsdruckkurven gezeigt. Für das Copolymer mit dem geringsten BzMA-Gehalt wurden geringfügig niedrigere Trübungsdrücke als für Polyethylen gemessen, während für die anderen Proben mit steigendem Methacrylatgehalt eine deutlich größere Zunahme der Trübungsdrücke als für die bisher vorgestellten Ethen-(Meth)Acrylat-Copolymersysteme gefunden wird. Das 
zugehörige Homopolymer Polybenzylmethacrylat ist bei einem Polymergewichtsbruch von $5 \%$ in Ethen innerhalb der experimentellen Grenzen (3000 bar, $\left.260^{\circ} \mathrm{C}\right)$ unlöslich.

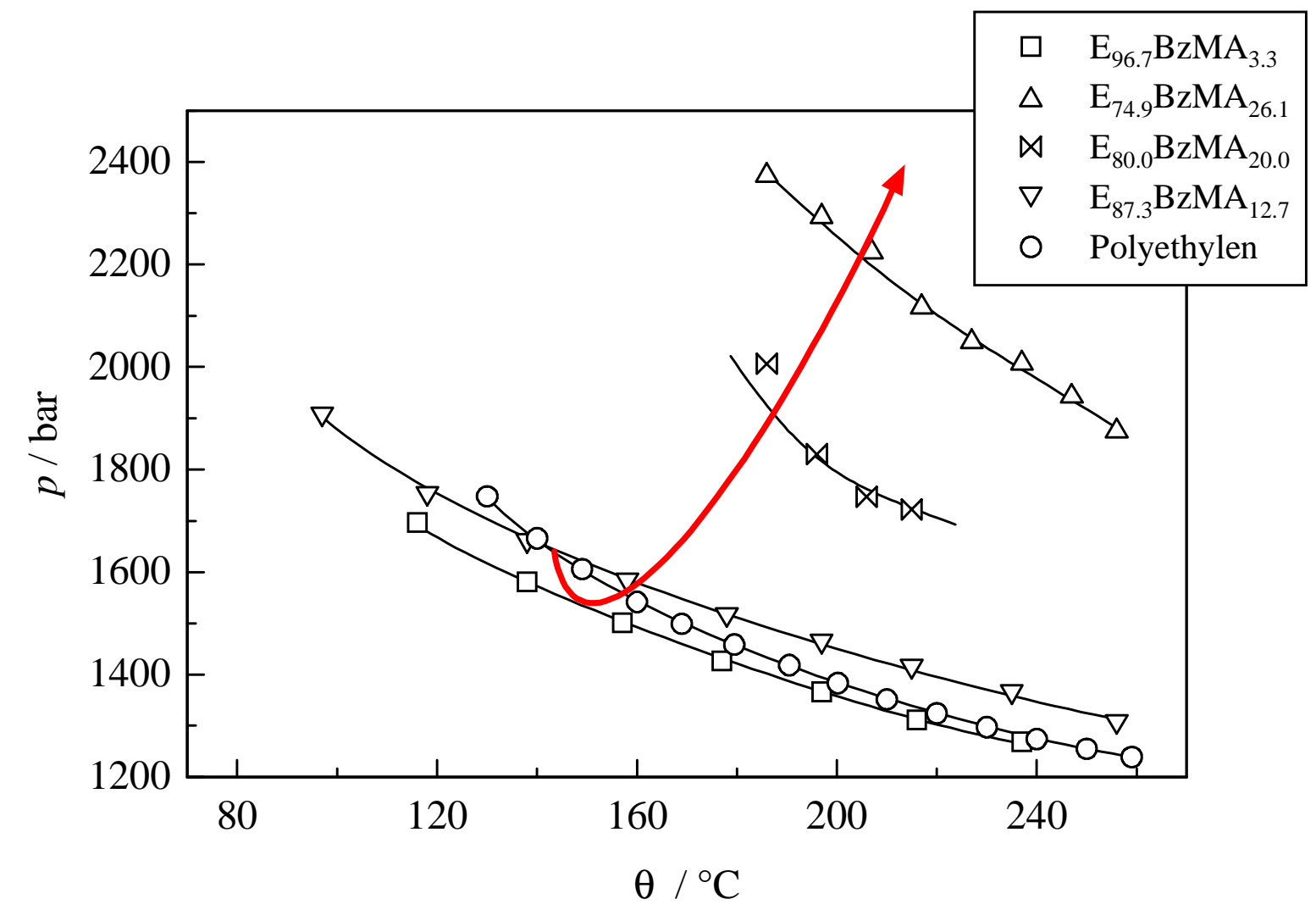

Abbildung 5.13: Trübungsdruckkurven für Mischungen aus Ethen und Ethen-Benzylmethacrylat-Copolymeren mit verschiedenem BzMA-Gehalt.

Diese Ergebnisse erstaunen zunächst, weil im bisher entwickelten Erklärungsmuster für das Entmischungsverhalten der Ethen-(Meth)Acrylat-Copolymere davon ausgegangen wird, dass zum einen die voluminösen Benzyl-Gruppen die Carboxylfunktionen der Comonomereinheiten sehr gut abschirmen und zum anderen eine relativ große Oberflächenvergrößerung der Copolymere durch den Einbau von BzMA entsteht. Beide Effekte sollten in Richtung Erniedrigung des Trübungsdrucks zielen.

Es ist unwahrscheinlich, dass der Grund für die bei diesem System beobachteten relativ hohen Trübungsdrücke allein bei den Dipol-Dipol-Wechselwirkungen der polaren Carboxylat-Funktionalitäten zu finden ist. Vermutlich tritt bei E-BzMA-Copolymeren bei Zunahme des Methacrylatgehalts der sogenannte „stacking“-Effekt der aromatischen Esterreste auf. Hierbei nähern sich die aromatischen Spezies entweder coplanar parallel (,face to face“) oder T-förmig (,side to face“) an. Der energetische Vorteil dieser Ausrichtung liegt in der Wechselwirkung zwischen elektronenreichen $\pi$-Orbitalen der 
aromatischen Gruppen [17,18]. Aus der Literatur ist bekannt, dass der sogenannte „stacking“-Effekt sowohl inter- als auch intramolekular auftreten kann [19]. Daher sollte mit zunehmendem Gehalt an Comonomeren mit aromatischer Funktionalität ein Ansteigen der Wechselwirkungen innerhalb eines Makromoleküls eintreten. Die an intramolekularen Wechselwirkungen beteiligten Spezies tendieren dazu, den für die Ausbildung der Wechselwirkung optimalen Abstand einzunehmen. Bei Veränderung des Abstands zwischen den aromatischen Gruppen ändert sich die magnetische Umgebung der Atomkerne dieser Molekül. Dies äußert sich durch eine Änderung der Resonanzfrequenz im NMR-Spektrum. Bei Annäherung der aromatischen Systeme aneinander wird zunächst eine sog. Entschirmung (Schwächung des lokalen Magnetfelds) der Kerne und eine Hochfeldverschiebung der entsprechenden Kernresonanzsignale gefunden. Bei noch geringerem Abstand kehrt sich dieser Effekt um, so dass das lokale Magnetfeld verstärkt wird und die Resonanzfrequenzen tieffeld-verschoben im Spektrum erscheinen. Becker [20] hat die im Rahmen dieser Arbeit hergestellten E-BzMA-Copolymeren ${ }^{1}$ H-NMR-spektroskopisch untersucht und eine erheblich mit dem BzMA-Gehalt der Proben zunehmende Hochfeldverschiebung der Resonanzfrequenzen der aromatischen Protonen festgestellt. Diese Beobachtung unterstützt die Annahme, dass intramolekulare $\mathrm{p} \pi$-p $\pi$-Wechselwirkung bei zunehmenden Gehalt an Comonomer die Ursache für die verschlechterte Löslichkeit der EBzMA-Copolymere darstellt, obwohl aufgrund der hohen Polarisierbarkeit von aromatischen Spezies eine gute Löslichkeit durch Copolymer-Solvens-Dispersionswechselwirkungen zu erwarten gewesen wäre.

\subsection{Kontinuierliche Trübungsdruckmessungen mit der ,inline"-Methode}

In Abschnitt 3.3.5 wurde ein modifizierter experimenteller Aufbau zur kontinuierlichen Bestimmung von Trübungsdrücken (,inline-Methode“) vorgestellt. Mit dieser Technik wird der Entmischungsdruck der Reaktionsmischung unmittelbar nach Verlassen des Synthesereaktors in einer nachgeschalteten optischen Hochdruckzelle und damit unter besonders praxisnahen Bedingungen gemessen. Die Reaktionsmischung ist abgesehen von möglicherweise eingesetzten Molmassenreglern oder geringen Mengen Initiator ein ternäres Gemisch aus Copolymer, Ethen und je nach gewählter Comonomerdosierung unterschiedlichen Mengen an nicht abreagiertem Comonomer. Es ist bekannt, dass die Anwesenheit von Comonomeren des Typs Acrylsäureester den Trübungsdruck der entsprechenden ternären Systeme z.T. erheblich absenkt (sog. Cosolvenswirkung). Somit sind die an der Reaktions- 
mischung bestimmten Trübungsdrücke für die Anwendungstechnik besonders wertvolle Daten.

Um die mit der Satzzelle ermittelten Trübungsdrücke der binären Systeme mit den Daten aus der kontinuierlichen Anlage (ternäre Systeme) vergleichen zu können, ist es erforderlich die Größe der Trübungsdruckabsenkung durch den Gehalt an freiem Comonomer zu kennen. Für die chemisch eng verwandten ternären Systeme Ethen/BA/E-BA und Ethen / EHA / E-EHA wurde ein linearer Zusammenhang zwischen der Absenkung des Drucks und der Konzentration an freiem Comonomer in der Reaktionsmischung gefunden [21]. Für eine experimentelle Quantifizierung dieses Zusammenhangs mit der „inline“Methode können Trübungsdruckmessungen unter Variation der Comonomerkonzentration ausgeführt werden. Dies lässt sich durch Zudosieren zusätzlicher Mengen Comonomer zur Reaktionsmischung nach Verlassen des Synthesereaktors erreichen. Aus der so bestimmbaren Abhängigkeit lässt sich der Trübungsdruck für verschwindende Mengen an freiem Monomer extrapolieren. In dieser Arbeit wurden die Systeme Ethen / Ethylmethacrylat / Ethen-Ethylmethacrylat-Copolymer und Ethen / Benzylmethacrylat / Ethen-Benzylmethacrylat-Copolymer mit der ,inline“-Technik untersucht. Die Messungen wurden jeweils bei konstanter Temperatur $\left(200^{\circ} \mathrm{C}\right)$ durchgeführt, mit dem Ziel innerhalb einer Versuchsreihe die Abhängigkeit des Trübungsdrucks möglichst vieler Copolymerproben von der Zusammensetzung bestimmen zu können.

\subsubsection{System Ethen / Ethylmethacrylat / Ethen-Ethylmethacrylat- Copolymer}

In Abbildung 5.14 sind die für das ternäre System E / EMA / E-EMA erhaltenen Resultate dargestellt. Aufgetragen ist der Trübungsdruck bei $200^{\circ} \mathrm{C}$ in Abhängigkeit vom Gewichtsanteil an Ethylmethacrylat-Monomer in der Mischung. Hierbei entspricht der Datenpunkt mit dem jeweils geringsten EMA-Anteil in der Monomermischung dem Trübungsdruck der Reaktionsmischung ohne Zudosieren von weiterem Comonomer nach dem Reaktor. Die Comonomerkonzentration ergibt sich dabei allein aus der Gleichgewichtskonzentration bei dem entsprechenden Umsatz. Die Trübungsdrücke für höhere Comonomergehalte in der Reaktionsmischung können nach Zudosieren weiteren Comonomers (s.o.) gemessen werden. Aus der Auftragung ist gut zu erkennen, dass analog zu den Literaturergebnissen für E / MA / EMA [21] für alle fünf Versuchsreihen ebenfalls ein in guter Näherung linearer Zusammenhang zwischen der Konzentration freien Monomers und dem Trübungsdruck existiert. 

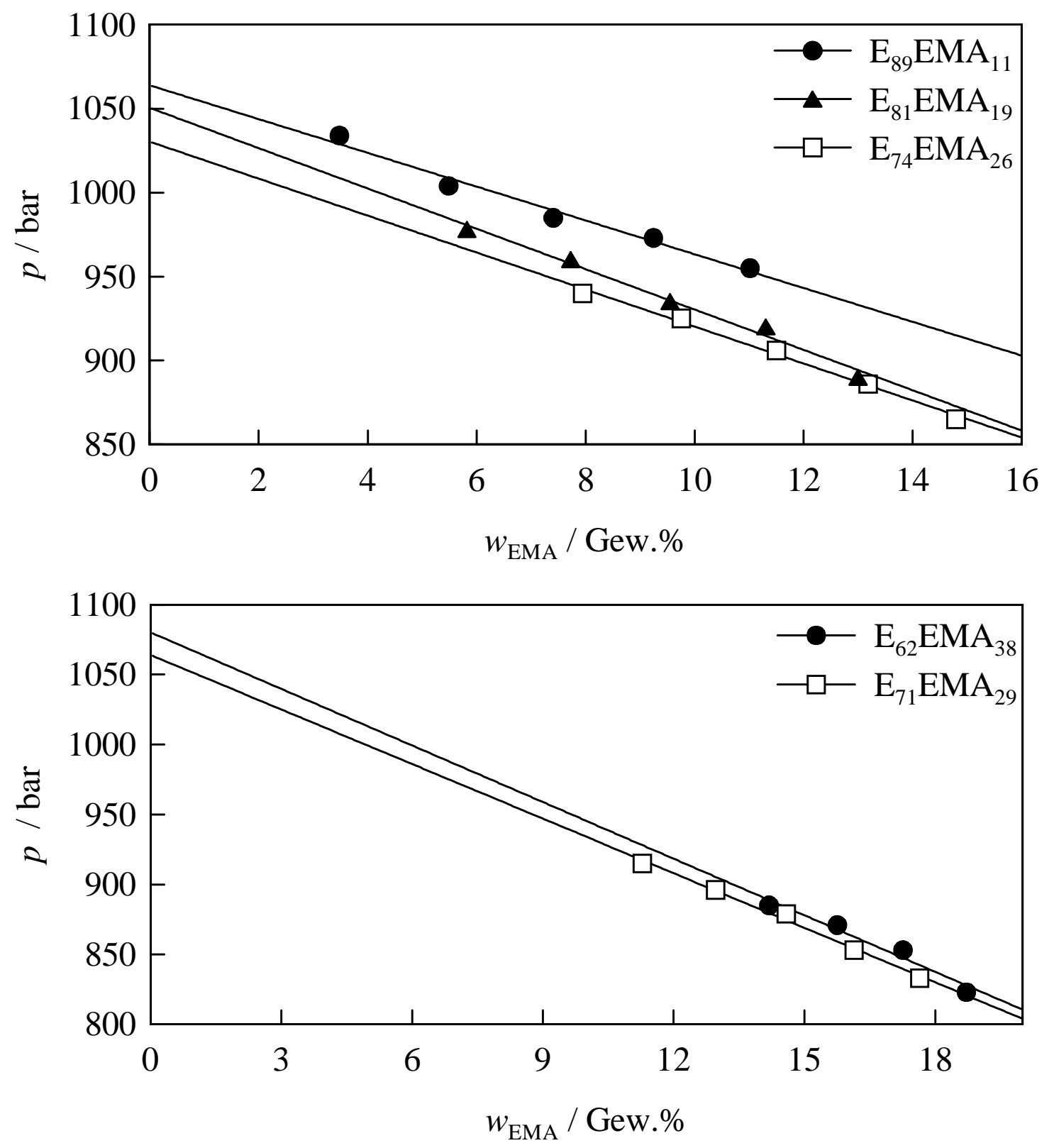

Abbildung 5.14: Abhängigkeit des Trübungsdrucks vom EMA-Gewichtsanteil ( $w_{\text {EMA }}$ ) in der Monomermischung für E-EMA-Copolymere unterschiedlicher Zusammensetzung.

Durch lineare Regression der experimentellen Datenpunkte und Extrapolation lässt sich der Trübungsdrücke des binären Systems Ethen / E-EMA aus dem Ordinatenabschnitt erhalten. Aus der Steigung der Ausgleichsgeraden lässt sich die spezifische Trübungsdruckabsenkung (s.u.) bestimmen. Diese ist ein Maß für die Wirksamkeit des betrachteten Comonomers als Cosolvens. 


\subsubsection{System Ethen / Benzylmethacrylat / Ethen-Benzylmethacrylat- Copolymer}

Die am Beispiel von fünf E-BzMA-Proben für das ternäre System E / BzMA / E-BzMA gemessene Abhängigkeit des Trübungsdrucks vom BzMA-Gehalt in der Comonomermischung ist in Abbildung 5.15 aufgetragen.

Analog zu dem System E/EMA/E-EMA wird auch hier eine lineare Abhängigkeit gefunden. Es fällt allerdings auf, dass die Steigung der durch lineare Regression erhaltenen Ausgleichsgeraden - gleichbedeutend mit der Größe des trübungsdrucksenkenden Effekts monomeren Benzylmethacrylats - mit dem BzMA-Anteil im Copolymer zunimmt.

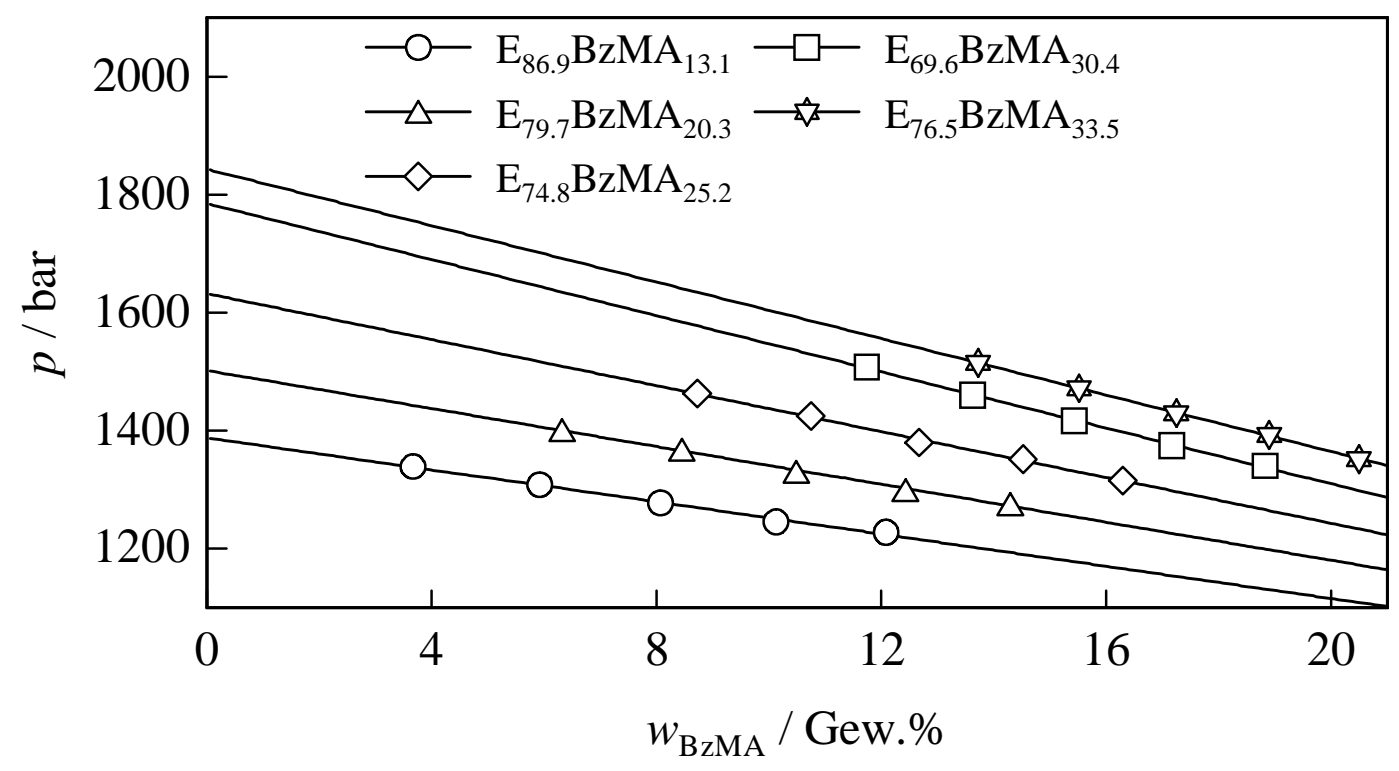

Abbildung 5.15: Abhängigkeit des Trübungsdrucks vom BzMA-Gewichtsanteil (w $\left.w_{B z M A}\right)$ in der Monomermischung für E-BzMA-Copolymere unterschiedlicher $\mathrm{Zu}$ sammensetzung.

In der Literatur wird der Mechanismus von polaren Cosolventien wie z.B. den Acrylaten bezü-glich ihrer trübungsdrucksenkenden Wirkung z.T. damit erklärt, dass die Cosolventien die Wechselwirkungen durch zusätzliche Dipol-Dipol-Kräfte zwischen polaren Polymerbausteinen und den Lösungsmittelanteil Cosolvens verbessern. Interessanterweise wurde in neueren Arbeiten [12,21] allerdings gezeigt, dass das Ausmaß der trübungsdrucksenkenden Wirkung verschiedener Cosolventien für Mischungen aus Ethen und EthenAcrylat-Copolymer nicht mit dem Dipolmoment des Cosolvens sondern vielmehr mit dessen Molekülgröße korreliert. In diesem Zusammenhang erscheint es lohnend, die spezifische Trübungsdruckabsenkung $\partial p / \partial f_{\mathrm{A}}$ in Abhängigkeit von der Copolymerzu- 
sammensetzung, zu betrachten. Zahlenwerte für die spezifische Trübungsdruckabsenkung $\partial p / \partial f_{\mathrm{A}}$ enthält man, wie bereits erwähnt, aus den Steigungen der jeweiligen Regressionsgeraden aus Auftragungen des Trübungsdrucks gegen den Stoffmengenanteil des Comonomers $f_{\mathrm{A}}$ in der Monomermischung (analog zu Abbildung 5.14 bzw. 5.15).

In Abbildung 5.16 ist die spezifische Trübungsdruckabsenkung für die beiden in dieser Arbeit untersuchten Systeme E / EMA / E-EMA und E / BzMA / E-BzMA als Funktion der Copolymerzusammensetzung im Vergleich zu Literaturdaten für die chemisch verwandten Systeme E / MA / E-MA und E / BA / E-BA dargestellt. Die Grafik zeigt somit, wie sich die trübungsdrucksenkende Wirkung von (Meth)Acrylaten in der Comonomermischung in Abhängigkeit von der Copolymerzusammensetzung ändert.

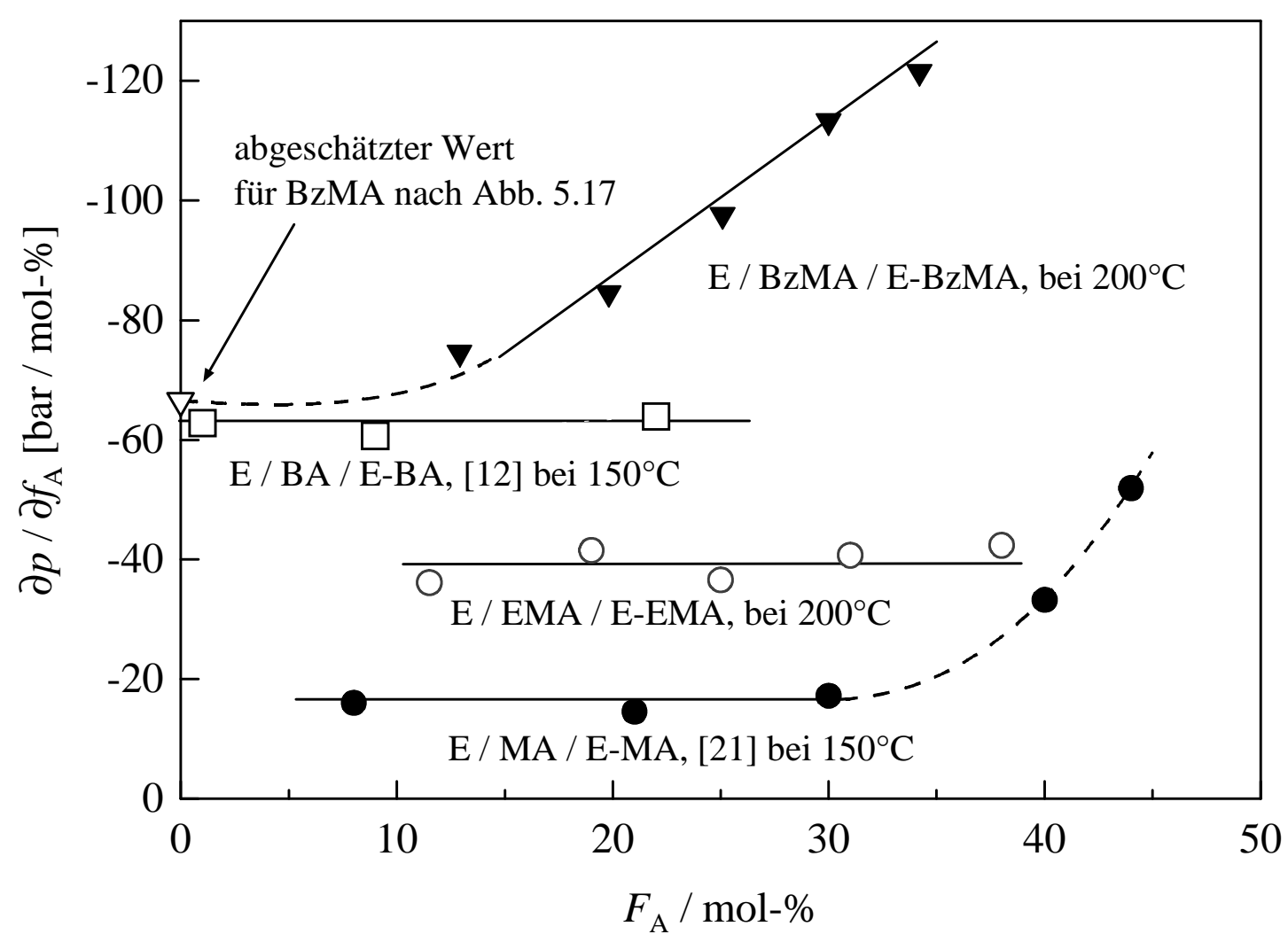

Abbildung 5.16: Spezifische Trübungspunktabsenkung verschiedener ternärer Systeme in Abhängigkeit von der Copolymerzusammensetzung

Anzumerken ist, dass für die Messungen in dieser Arbeit eine Temperatur von $200^{\circ} \mathrm{C}$ gewählt wurde, wohingegen die Literaturergebnisse bei $150^{\circ} \mathrm{C}$ bestimmt wurden. Aus [12] ist jedoch bekannt, dass die Temperaturabhängigkeit der spezifische Trübungsdruckabsenkung $\partial p / \partial f_{\mathrm{A}}$ in Temperaturbereich von $150-200^{\circ} \mathrm{C}$ vernachlässigbar gering ist, so dass die Vergleichbarkeit der Resultate gegeben ist. Aus Auftragung 5.16 ist ersichtlich, 
dass die Werte für die spezifische Trübungspunktabsenkung für E / EMA / E-EMA gerade zwischen denen für E/MA/E-MA und E/BA/E-BA liegen. Außerdem lässt sich erkennen, dass der trübungs-drucksenkende Effekt für E / EMA / E-EMA im untersuchten Zusammensetzungsbereich genauso wie für $\mathrm{E} / \mathrm{BA} / \mathrm{E}-\mathrm{BA}$ und im Bereich geringer Acrylatgehalte für E/MA / E-MA nicht vom (Meth)Acrylatgehalt im Polymer abhängt. Angemerkt wird, dass auch für das hier nicht gezeigte System Ethen-EthylhexylacrylatCopolymer (E-EHA) [21], keine Abhängigkeit gefunden wird.

Für das E / BzMA / E-BzMA-System und für E / MA / E-MA bei höheren Methylacrylatgehalten nimmt die spezifische Trübungsdruckabsenkung mit der Copolymerzusammensetzung zu. In Abbildung 5.16 wird dies durch die, jeweils in diesem Bereich gestrichelt gezeichneten Ausgleichskurven für die Messwerte verdeutlicht. Im Falle von E / MA / EMA korreliert das Einsetzen dieser Abhängigkeit gerade mit dem zu höheren Comonomergehalten (des Copolymeren) gefundenen Anstieg der Trübungsdrücke im binären System E / E-MA. Durch den mit steigendem Methylacrylatgehalt im statistischen Copolymeren kleiner werdenden Abstand zwischen polaren Acrylateinheiten nehmen intra- und intermolekulare, die Löslichkeit verschlechternde Carboxylat-Carboxylat-Wechselwirkungen zu. Die Zugabe polarer Cosolvensmoleküle beeinflusst dies, indem der Anteil löslichkeitsverbessernder Polymersegment-Monomer-Wechselwirkungen ansteigt. Ähnliche Effekte sollten das analoge Verhalten im System E/BzMA / E-BzMA erklären. Die im untersuchten Zusammensetzungsbereich beobachtete Abhängigkeit der Trübungsdruckabsenkung von $F_{\mathrm{BzMA}}$ wird sich zu kleinen $F_{\mathrm{BzMA}}-$ Werten abschwächen. So sollte, entsprechend der Korrelation in Abb. 5.17, im Grenzfall $F_{\text {BzMA }}$ gegen 0 für die spezifische Trübungsdruckabsenkung ein Wert von etwa - 63 bar / mol-\% gelten. Dieser aus einer Abschätzung anhand der nachfolgend in Abbildung 5.17 dargestellten Korrelation erhaltene Wert (grau gefülltes Symbol) ist in Abbildung 5.16 als offenes Dreieck auf der Ordinate eingezeichnet. Der abgeschätzte Wert stimmt, wie in 5.16 erkennbar, unter Berücksichtigung der für E-BzMA zu erwartenden Abnahme der spezifische Trübungsdruckabsenkung zu geringen $F_{\mathrm{A}}$ gut mit den experimentellen Daten überein.

Die von der Zusammensetzung unabhängige Trübungspunktabsenkung durch Ethylmethacrylat unterstützt die These, dass ein Ethylesterrest bereits eine Größe hat, die ausreicht, effektiv die Dipol-Dipol-Wechselwirkungen einzelner Methacrylateinheiten in der Polymerkette abzuschirmen. Die Resultate zeigen auf, dass die spezifische Trübungsdruckabsenkung dann von der Copolymerzusammensetzung abhängt, wenn Wechsel- 
wirkungen zwischen den Comonomersegmenten das Löslichkeitsverhalten maßgeblich bestimmen.

Interessanterweise findet sich für die Absolutwerte der spezifischen Trübungsdruckabsenkung der Systeme in den Bereichen, in denen keine Abhängigkeit von $F_{\mathrm{A}}$ besteht, eine bemerkenswert gute Korrelation mit dem Molvolumen des reinen Cosolvens. Dies wird in Abbildung 5.17 in einer Auftragung der spezifischen Trübungsdruckabsenkung gegen des Molvolumen (bei 1 bar und $25^{\circ} \mathrm{C}$ ) illustriert.

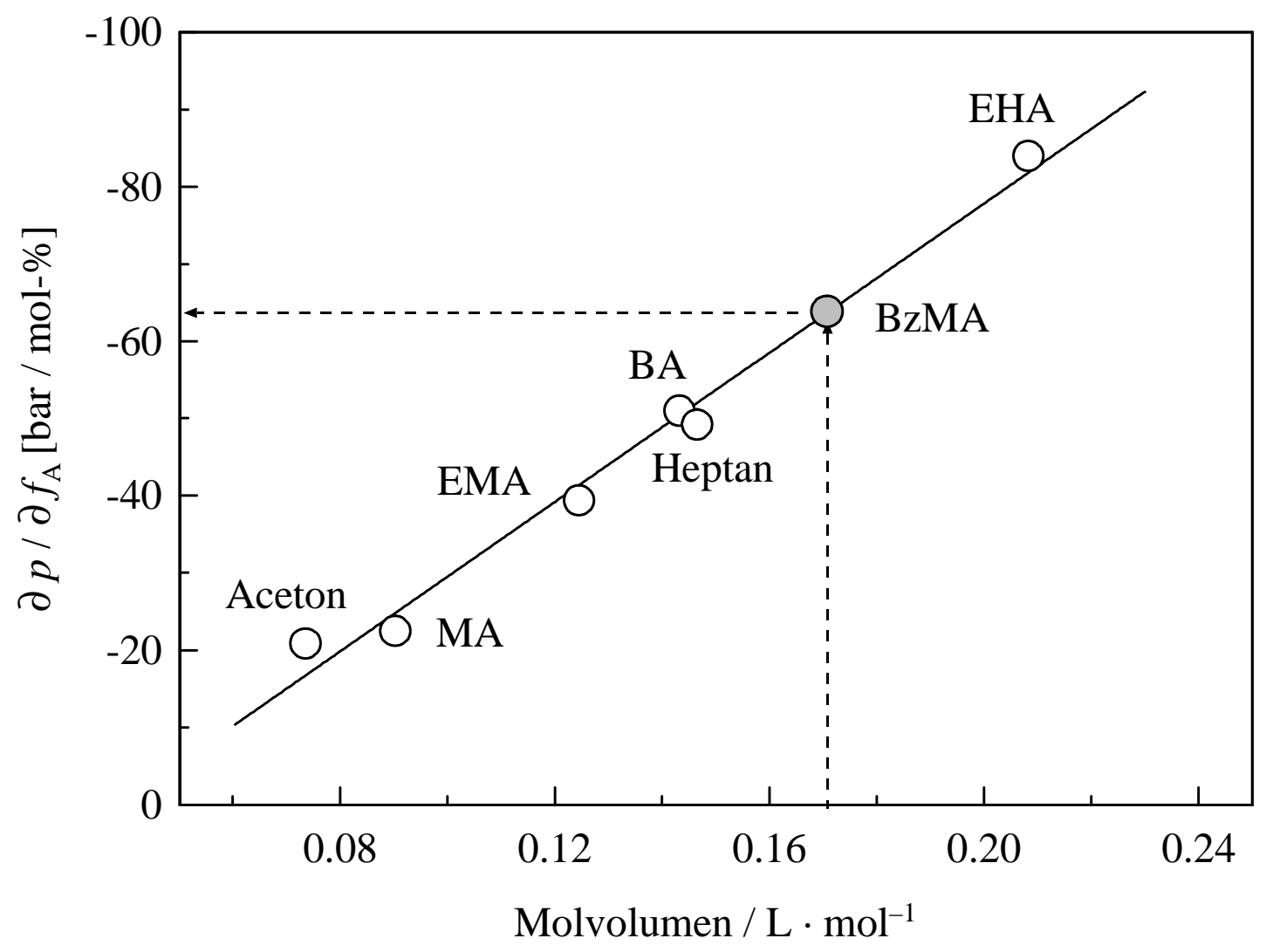

Abbildung 5.17: $\quad$ Spezifische Trübungsdruckabsenkung verschiedener Cosolventien in Abhängigkeit von ihrer Molekülgröße; EMA [diese Arbeit], die übrigen Werte sind Ref. [12] entnommen.

Hierbei ist darauf hinzuweisen, dass die Werte aus der Literatur für die Solventien Aceton und Heptan für Mischungen aus Ethen und einem E-MA-Copolymer bestimmt wurden. In der Literatur wird im Zusammenhang mit der durch Cosolventien verbesserten Löslichkeit von Polyethylen und Copolymeren häufig der Begriff der sogenannten "Differenz des Freien Volumen" von Polymer und Lösungsmittel erwähnt [22, 23]. Das Freie Volumen wird dabei als das von einem Molekül zur Rotation und Translation benötigte Volumen angesehen, wobei die thermische Ausdehnung als qualitatives Maß gesehen wird [24]. 
Danach erhöht ein (Meth)Acrylat-Cosolvens aufgrund seiner höheren Dichte die Gesamtdichte der Mischung, reduziert damit den Unterschied zwischen dem Freien Volumen von Solvens und Polymer und verbessert die Löslichkeit. Der Effekt ist begründet in der negativen nichtkombinatorischen Entropie, die beim Mischen eines Stoffs vergleichsweise geringer Dichte mit einem dichteren Stoff zu erwarten ist und die mit dem Dichteunterschied der Mischungspartner zunimmt. Diese Überlegungen können die in Abbildung 5.17 dargestellte Korrelation der spezifischen Trübungsdruckabsenkung mit dem molaren Volumen des Cosolvens erklären, wenn man annimmt, dass die Dichte des Polymeren deutlich von der des Lösemittels verschieden ist. Die gefundene gute Korrelation ermöglicht es allein über die Ermittlung der Cosolvensdichte bzw. des Cosolvens-Molvolumens eine Aussage über die Größe der durch das Cosolvens bewirkten Beeinflussung des Trübungsdrucks zu treffen. Im Rahmen dieser Annahme würde auch verständlich, dass die Wirkung eines Cosolvens-Materials auf den Trübungsdruck unterschiedlicher Copolymere recht ähnlich ist (solange keine spezifischen Segment-Wechselwirkungen den Trübungsdruck bestimmen).

\subsection{Ergebnisse der Trübungsdruckmessungen an Mischungen aus Ethen und Ethen-Methacrylsäure-Butylmethacrylat- Terpolymeren}

In dieser Arbeit wurden zum ersten Mal Trübungsdrücke für ternäre Ethen-(Meth)Acrylsäureester-(Meth)Acrylsäure-Copolymere bestimmt. Zum einen erschien es prinzipiell interessant, wie sich die beiden Comonomertypen (Meth)Acrylat und (Meth)Acrylsäure, welche bei Einbau als Comonomere in den binären Randsystemen Ethen-(Meth)Acrylatund Ethen-(Meth)Acrylsäure-Copolymer eine gegensätzliche Wirkung auf die Entmischungsdrücke haben, kombiniert in einem Terpolymer auf den Entmischungsdruck auswirken. Zum anderen war es reizvoll zu testen, ob die außerordentlich hohen Trübungsdrücke der Ethen-(Meth)Acrylsäure-Copolymere [25, 26] durch den Einbau von (Meth)Acrylat-Bausteinen verringert werden können. Um hierbei einen möglichst großen Effekt zu erzielen, wurde als (Meth)Acrylat-Comonomer BMA gewählt, welches als Comonomer in Ethen-Copolymeren besonders niedrige Trübungsdrücke bewirkt (vgl. Abschnitt 5.2.4). Als Säure-Comonomer wurde Methacrylsäure verwendet, da für das Randsystem EthenMethacrylsäure-Copolymer ein umfangreicher Trübungsdruckdatensatz existiert [25]. 
Zum Erhalt von Polymermaterial für die thermodynamischen Messungen war zunächst vorgesehen ausgehend von Ethen-(Meth)Acrylsäure-Copolymeren E-MAS-BMA-Terpolymere durch polymeranaloge Umsetzung herzustellen. Diese Strategie ermöglicht es, polymere Verbindungen für die thermodynamischen Studien einzusetzen, die in ihrer Mikrostruktur, also in der Gesamtzahl funktioneller Gruppen, in der chemischen Einheitlichkeit, im Verzweigungsgrad und in der Molmassenverteilung vollständig identisch sind und sich nur in den Anteilen der beiden funktionellen Gruppen (Ester und Säure) unterscheiden. Besonders gute Alkylierungsmittel, mit welchen schon bei Zimmertemperatur effektiv Carbonsäuren zu Alkyestern umgesetzt werden können, sind die Diazoalkane. Diazomethan als auch Diazoethan haben sich für polymeranaloge Umwandlungen als gut geeignet erwiesen [27]. Veresterungen mit Diazoalkanen weisen zudem den besonderen Vorteil auf, dass überschüssiges Alkylierungsmittel leicht entfernt werden kann, da Diazoalkane schnell zu Stickstoff und den entsprechenden Alkanen zerfallen. Es sei angemerkt, dass in dieser Arbeit Diazomethan auch im Rahmen der Probenvorbereitung für die NMR-Spektroskopie zur Umwandlung von polymeren Säure- zu Methylestergruppen verwendet wurde (vgl. Abschnitt 3.2.3). Für die hier vorgesehene Teilveresterung von Ethen-Methacrylsäure-Copolymeren sollten sich Diazoalkane jedoch weniger eignen. Dies liegt daran, dass es angesichts der außerordentlich hohen Reaktivität der Diazoalkane schwierig ist, einen statistisch verteilten Anteil an polymeren Säuregruppen umzusetzen, da die Reaktion nahezu instantan abläuft. Problematisch ist hierbei auch, dass die zu veresternden Ethen-Säure-Copolymere aufgrund ihrer schlechten Löslichkeit bei der Reaktion nur in einem im Lösungsmittel aufgequollenen Zustand vorliegen (vgl. 3.2.3). In Kombination mit einer schnellen Veresterungsumsetzung sollte dies zu Produkten führen, die vornehmlich an der Oberfläche der Gelpartikel verestert sind.

Eine vom Prinzip geeignete Veresterungsmethode um Terpolymere mit einem statistisch verteilten Anteil veresterter Säureeinheiten und damit ein chemisch einheitliches Produkt zu erhalten, sollte am ehesten durch eine in homogener Phase ablaufende und zu einem Gleichgewicht führende Reaktion gegeben sein. Auf Basis dieser Vorüberlegung wurden im Rahmen dieser Arbeit verschiedene klassische Veresterungsmethoden getestet. Als Edukt wurde für alle Experimente ein kommerzielles E-MAS-Terpolymer mit einem MAS-Gehalt von 7 mol-\% (Nucrel, DuPont) eingesetzt. Die Reaktionen wurden in den Lösungsmitteln 1,2,4-Trichlor-benzol und Toluol ausgeführt, in denen E-MAS-Copolymere oberhalb von ca. $100^{\circ} \mathrm{C}$ löslich sind. Butanol wurde als Alkoholkomponente verwendet und $p$-Toluolsulfonsäure als saurer Katalysator. In allen Fällen konnten nur sehr geringe Säureanteile 
umgesetzt werden. Trotz Anwendung des Edukts Butanol im mehrfachen Überschuss und der Entfernung des Reaktionsprodukts Wasser aus dem Gleichgewicht mit einem Wasserabscheider und dem Ausdehnen der Reaktionszeit auf $76 \mathrm{~h}$ gelang es nicht, mehr als 1 mol-\% der Säuregruppen des Copolymers zu verestern. Als Ursache für den z.T. niedrigen Gleichgewichtsumsatz bei polymeranalogen Umsetzungen werden häufig Nachbargruppeneffekte diskutiert [27].

In Abbildung 5.18 sind die für drei aus Teilveresterungen von E-MAS erhaltenen EthenBMA-MAS-Terpolymere bestimmten Trübungsdruckkurven dargestellt.

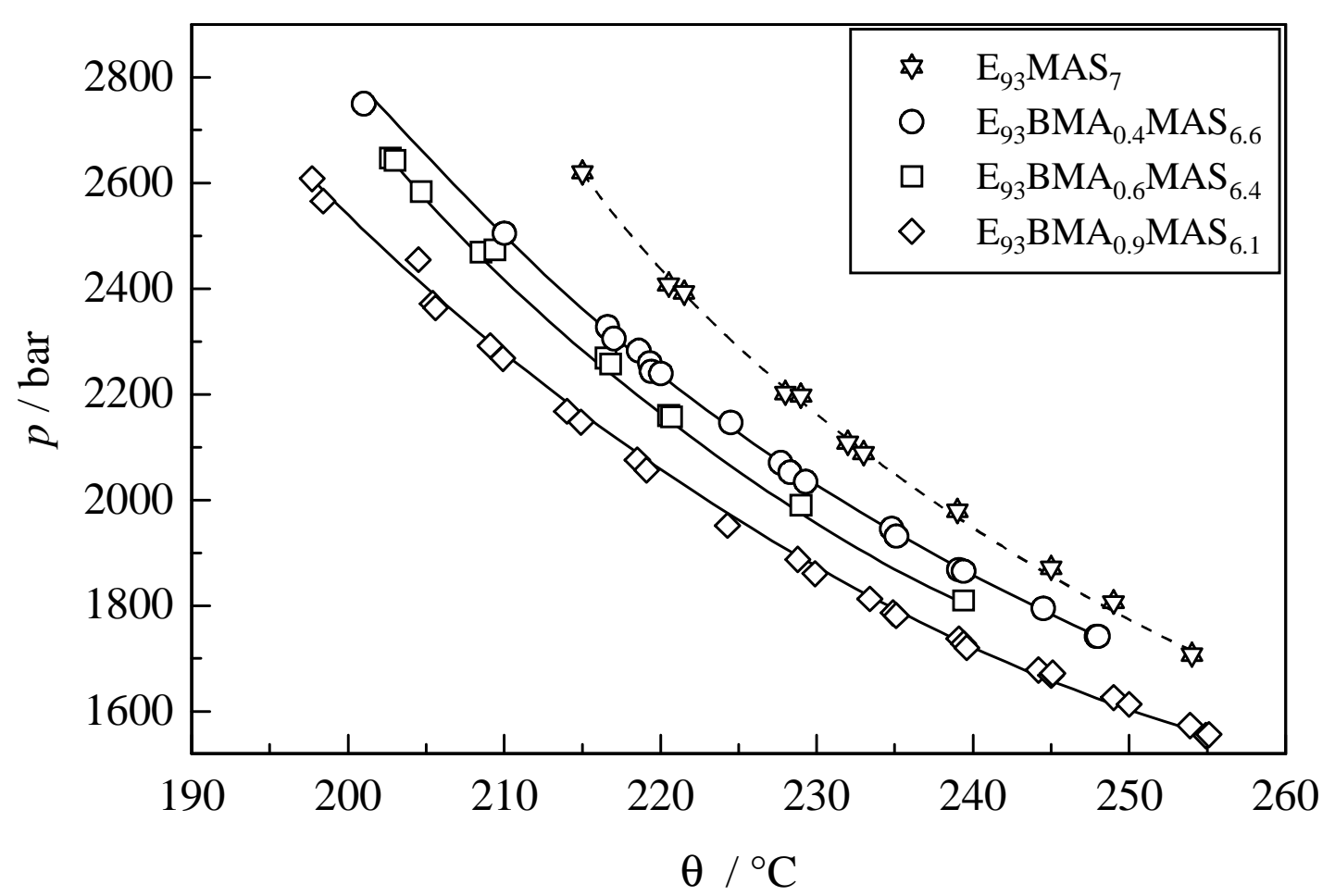

Abbildung 5.18: Trübungsdruckkurven für ein Ethen-Methacrylsäure-Copolymer sowie verschiedene Ethen-Butylmethacrylat-Methacrylsäure-Terpolymere welche durch Verestern des E-MAS-Copolymers mit Butanol erhalten wurden. Der Gesamtcomonomergehalt der Proben beträgt jeweils 7 mol-\%.

Der Comonomergehalt der Proben ist in der Auftragung als Subskript angegeben. Die Trübungskurve der Probe, welche als Edukt für die Veresterungen eingesetzt wurde, ist gestrichelt dargestellt. Es wird deutlich, dass der Trübungsdruck trotz des nur sehr geringen Anteils zu Esterfunktion umgewandelter Säuregruppen um bis zu 500 bar abgesenkt wird. 
Aufgrund der beschriebenen Schwierigkeiten, E-BMA-MAS-Terpolymere in einem weiten Zusammensetzungsbereich durch polymeranaloge Umsetzungen herzustellen, wurde ein Satz Terpolymerproben durch direkte Hochdruck-Terpolymerisation hergestellt (vgl. Abschnitt 3.1.4). Hierfür wurden in Vorexperimenten Monomerflüsse bestimmt, mit denen Terpolymere mit einem Gesamtcomonomergehalt von 7 mol-\% erhalten werden, welche aber unterschiedliche Anteile von Ester- und Säuregruppen aufweisen. Während der Messreihen zur Hochdrucksynthese der Terpolymere wurden jeweils auch Proben der Randsysteme E-MAS-Copolymer und E-BMA-Copolymer hergestellt. Obwohl bei allen Reaktionen identische Temperaturen und Verweilzeiten und sehr ähnliche Umsatze herschten ist der Gesamtcomonomergehalt der durch radikalische Hochdruckpolymerisation synthetisierten E-BMA-MAS-Terpolymere sowie die Polymermikrostruktur im Unterschied zu denen aus der Synthese durch polymeranaloge Umsetzung sicher nicht vollständig identisch. Da der Entmischungsdruck für E-BMA-MAS-Copolymere, wie aus Abbildung 5.19 ersichtlich, offenbar maßgeblich durch den Säuregehalt bestimmt wird, sollten die geringen Unterschiede in der Mikrostruktur der durch direkte Terpolymerisation erhaltenen Proben die Aussagekraft der Trübungsdruckmessungen nicht beeinträchtigen.

In Abbildung 5.19 sind die Trübungsdruckkurven von E-BMA-MAS-Terpolymeren dargestellt, welche durch Hochdrucksynthese erhaltenen wurden. Während der Trübungsdruck der E-BMA-Copolymerprobe nur eine geringe Temperaturabhängigkeit zeigt, fallen die Trübungsdrücke der Terpolymere und des E-MAS-Copolymers mit der Temperatur deutlich stärker ab. Bei tiefen Temperaturen wird eine besonders starke Temperaturabhängigkeit der Trübungsdrücke der Terpolymere beobachtet. Die Unterschiede in der Temperaturabhängigkeit der einzelnen Terpolymere korrelieren mit dem Säuregehalt. Dies beruht darauf, dass die hohen Trübungsdrücke säurehaltiger Polymere maßgeblich durch (stark temperaturabhängige) Wasserstoffbrückenbindungen und elektrostatische Wechselwirkungen [4,28] verursacht werden. Die Charakteristik des Verlauf der Kurven mit zunehmendem Säuregehalt ähnelt stark dem für das Randsystem E-MAS-Copolymer [25] Dies macht deutlich, dass das Entmischungsverhalten der Terpolymere maßgeblich durch den Säuregehalt bestimmt wird. 


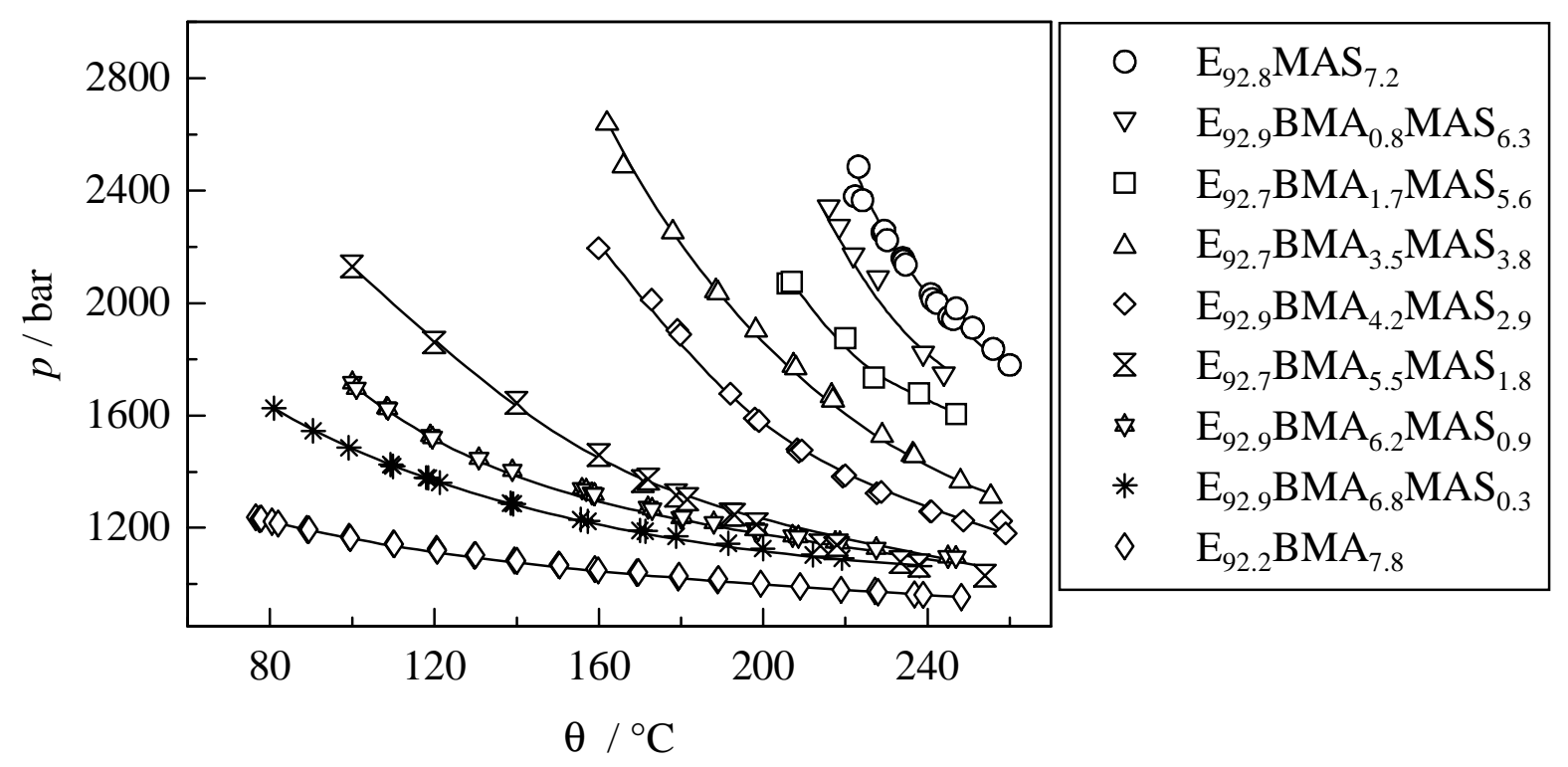

Abbildung 5.19: Einfluss des BMA- und MAS-Gehalt auf die Trübungsdruckkurven von Ethen-Butylmethacrylat-Methacrylsäure-Terpolymeren bzw. E-MAS und E-BMA-Copolymeren. Der Gesamtcomonomergehalt der Proben beträgt ca. 7 mol- $\%$.

Zur Ermittlung inwieweit der hohe Trübungsdruck der Ethen-MAS-Copolymere durch Einbau von zusätzlichem BMA gesenkt werden kann, sind im unteren Teil von Abbildung 5.20 Trübungskurven für zwei Copolymerproben mit einem Säuregehalt von ca. 1 mol-\% aufgetragen. Die grau gefüllten Quadratsymbole stehen für ein Methacrylsäurehaltiges Copolymer welches im Unterschied zu dem durch schwarz gefüllte Symbole repräsentierten E-MAS-Copolymer zusätzlich noch einen BMA-Gehalt von 6.2 mol-\% aufweist. Beim Vergleich der Kurven ist zu berücksichtigen, dass die in einer vorangegangen Arbeit bestimmten Daten für das E-MAS-Copolymer [25] für eine Copolymerkonzentration von $3 \%$ gelten. Auf der Basis von Literaturdaten zur Konzentrationsabhängigkeit des Trübungsdrucks des verwandten Systems Ethen/Ethen-Acrylsäure-Copolymer kann abgeschätzt werden, dass die Trübungsdrücke für $\mathrm{E}_{99.0} \mathrm{MAS}_{1.0}$ in dem in Abbildung 5.20 gezeigten Temperaturbereich für eine zu den Daten dieser Arbeit identische Polymerkonzentration von 5 Gew.\% etwa $25-45$ bar tiefer lägen. Es ist festzustellen, dass der zusätzliche BMAGehalt eine signifikante Senkung des Trübungsdrucks und eine erhebliche Verringerung der Temperaturabhängigkeit bewirkt. So beträgt der Unterschied unter Berücksichtigung des Einflusses der unterschiedlichen Polymerkonzentration bei $200^{\circ} \mathrm{C}$ ca. 250 bar. Interessant erscheint es nun zu klären, ob auch bei höheren Säuregehalten eine Reduzierung des Trübungsdruck durch Einbau von BMA möglich ist. Die bei hohen Gehalten besonders starke Abhängigkeit des Trübungsdrucks vom Säuregehalt erschwert den Vergleich der 
Trübungsdruckergebnisse für E-BMA-MAS-Terpolymere mit Literaturdaten zu E-MASCopolymeren. Die Problematik besteht darin, dass nicht vollständig sichergestellt werden kann, dass Abweichungen im Säuregehalt innerhalb der Genauigkeit der Gehaltsanalyse von ca. \pm 0.4 mol-\% nicht größere Effekte verursachen als derjenige aus dem zusätzlichen Einbau von Butylmethacrylat. Um bestmöglichst einheitliche Säuregehalte zu erreichen wurde daher so vorgegangen, dass im direkten Anschluss an die Synthese des E-MASCopolymers $\mathrm{E}_{92.8} \mathrm{MAS}_{7.2}$ bei gleichen Bedingungen (MAS-Feed, Initiator-Feed, Synthesetemperatur) innerhalb eines Syntheseexperiments zusätzlich der BMA-Gehalt variiert wurde. Hierbei wurden die Proben $\mathrm{E}_{86.2} \mathrm{MAS}_{7.3} \mathrm{BMA}_{5.5}$ und $\mathrm{E}_{79.6} \mathrm{MAS}_{7.1} \mathrm{BMA}_{13.3}$ erhalten. In Abbildung 5.20 sind die für diese Proben erhaltenen Trübungsdruckkurven dargestellt. Aus der Auftragung ist erkennbar, dass der Trübungsdruck von Ethen-MethacrylsäureCopolymeren auch bei hohen Säuregehalten durch den Einbau von BMA gesenkt werden kann.

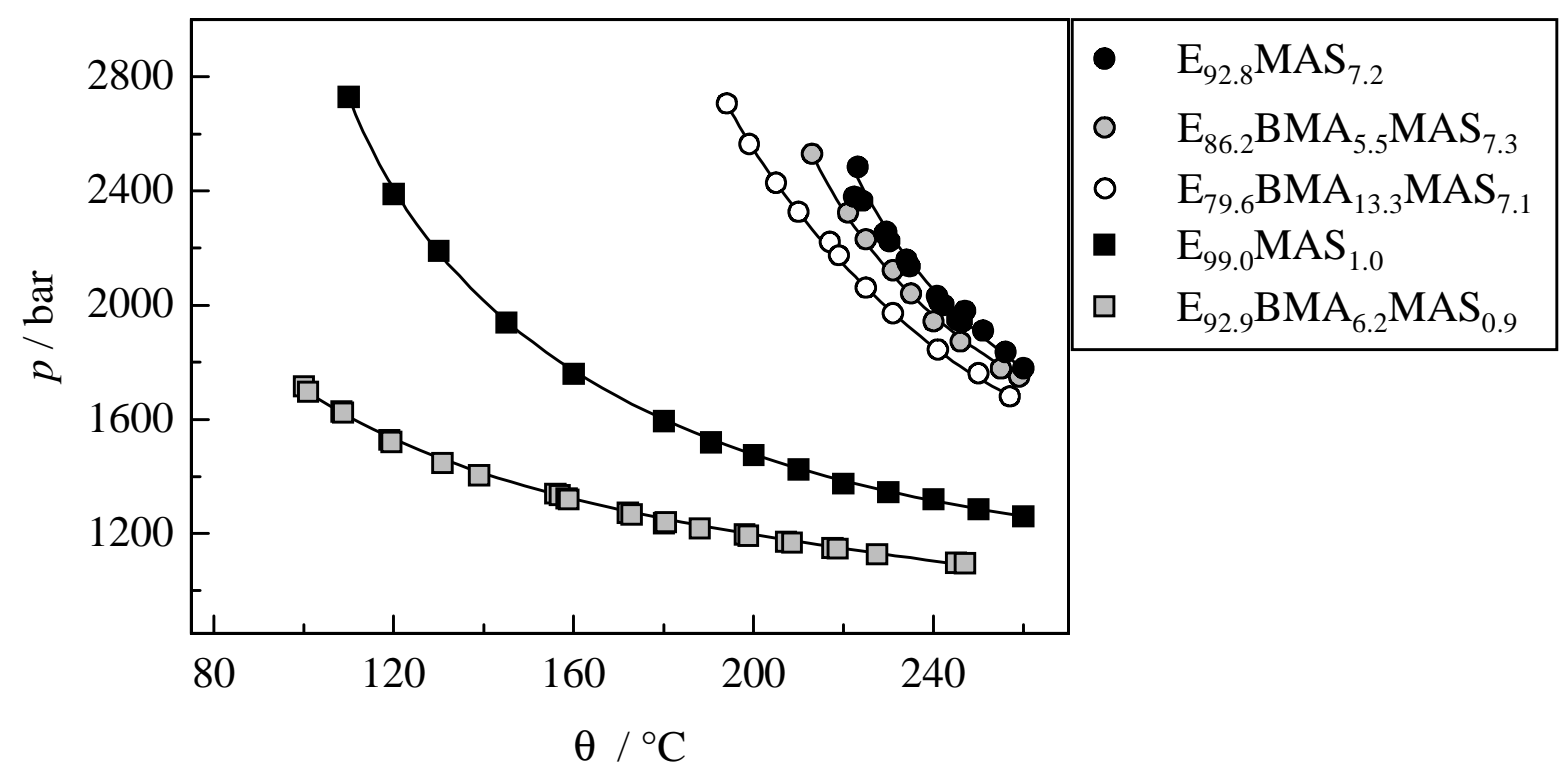

Abbildung 5.20: Effekt des zusätzlichen Einbaus von BMA auf den Trübungsdruck von Ethen-Methacrylsäure-Copolymeren. Trübungsdruckkurven für Mischungen aus Ethen und Ethen-Methacrylsäure-Copolymeren sowie für Mischungen aus Ethen und Ethen-Methacrylsäure-Butylmethacrylat-Terpolymeren.

Die durch den Einbau von 13.2 mol-\% BMA erzielte Reduzierung beträgt bei $220^{\circ} \mathrm{C}$ ca. 200 bar. Durch den Einbau von 5.5 mol-\% wird ein Senkung des Trübungsdrucks um lediglich ca. 60 bar erreicht. Mit in etwa dergleichen Menge eingebautem BMA wird bei identischer Temperatur für ein Copolymer mit einem Säuregehalt von 1 mol-\% der Trübungsdruck allerdings schon um etwas mehr als 200 bar gesenkt. Dieser Wert ist 
praktisch identisch mit der Trübungsdruckabsenkung welche bei gleicher Temperatur durch den Einbau etwa derselben Menge BMA für das Randsystem Polyethylen beobachtet wird (vgl. Abschnitt 5.4.4).

Zusammenfassend können folgende Erkenntnisse aus den in diesem Kapitel geschilderten Ergebnissen gewonnen werden:

1) Die Trübungsdruckkurven der Terpolymere fügen sich entsprechend ihrer Zusammensetzung zwischen den Kurven der Copolymer-Randsysteme ein. Dies gilt sowohl für die Trübungsdrücke, als auch für die Temperaturabhängigkeit der Trübungsdruckkurven.

2) Der Einbau unpolarer Gruppen (BMA-Einheiten) führt, wenn das Löslichkeitsverhalten bereits stark durch polare Wechselwirkungen dominiert ist, (Abb. 5.20; hoher MAS-Gehalt) nur zu einer geringen Löslichkeitsverbesserung. Es erscheint wahrscheinlich, dass der löslichkeitsverbessernde Effekt lediglich auf zunehmenden Dispersionswechselwirkungen unter Beteiligung der BMA-Einheiten mit dem Lösungsmittel Ethen beruht. Die Wasserstoffbrückenbindungen und polaren Wechselwirkungen der Säurefunktionen werden offenbar durch den Einbau von BMABausteinen nicht gestört bzw. reduziert. Für niedrigere MAS-Gehalte führt der Einbau von BMA-Comonomer $\mathrm{zu}$ einer deutlicheren Absenkung des Trübungsdrucks. Aufgrund der geringen Zahl der Säurefunktionalitäten können deren zwar starke, aber jetzt nur in geringem Ausmaß auftretenden Wasserstoffbrückenbindungen von den Dispersionswechselwirkungen an den BMA-Einheiten überkompensiert werden. 


\section{$5.7 \quad$ Literatur Kapitel 5}

[1] G. Sadowski, Habilitationsschrift, Technische Universität Berlin (1999)

[2] M. A. McHugh, V. J. Krukonis, Supercritical Fluid Extraction, 2ed. ButterworthHeinemann Boston (1994)

[3] R. L. Scott, P. B. van Konyenburg, Disc. Faraday Soc. 49 (1970) 87

[4] B. Folie, M. Radosz, Ind. Eng, Chem. Res. 34 (1995) 1501

[5] J. S. S. Toh, D, M. Huang, P. A. Lovell, R. G. Gilbert, Polymer 42 (2001) 1915

[6] L. Wild, Temperature Rising Elution Fractionation in Advances in Polymer Science (1998) Springer Verlag Berlin

[7] B. M. Hasch, S. H. Lee, M. A. McHugh, Fluid Phase Equil. 83 (1993) 341

[8] S. Saeki, N. Kuwahara, S. Konno, M. Kaneko, Macromolecules 6 (1973) 246

[9] C. Müller, Dissertation, Karlsruhe (1996)

[10] C. Müller, L. R. Oellrich, Acta Polymer. 47 (1996) 404

[11] R. W. Wind, Dissertation, Darmstadt (1992)

[12] H. D. Dietzsch, Dissertation, Göttingen (1999)

[13] Th. W. deLoos, W. Poot, G. A. M. Diepen, Macromolecules 16 (1983) 111

[14] M. Lora, F. Rindfleisch, M. A. McHugh, J. Appl. Polym. Sci. 73 (1999), 1979

[15] H.-S. Buyn, B. M. Hasch, M. A. McHugh, F.-O. Mähling, M. Busch, M. Buback Macromolecules 29 (1996) 1625

[16] E. Narr, Diplomarbeit, Göttingen (1999)

[17] C. A. Hunter, J. K. M. Sanders, J. Am. Chem. Soc. 112 (1990) 5525

[18] L. F. Newcomb, S. H. Gellmann, J. Am. Chem. Soc. 116 (1994) 4993

[19] S. Lahiri, J. L. Thompson, J. S. Moore, J. Am. Chem. Soc. 122 (2000) 11315

[20] F. Becker, Diplomarbeit, Göttingen (2002)

[21] T. Dröge, Dissertation, Göttingen (1997)

[22] D. Patterson, Polym. Eng. Sci. 22 (1982) 64

[23] F. Rindfleisch, T. P. DiNoia, M. A. McHugh, J. Phys. Chem. 100 (1996) 15581 
[24] P. C. Hiemenz, Polymer Chemistry - The Basic Concepts, Marcel Dekker, NY (1984)

[25] M. Buback, H. Latz, Macromol. Chem. Phys. 204 No.4 (2003) 638

[26] S. H. Lee, M. A. McHugh, Polymer 6 (1996) 1317

[27] O. H. Möller, Dissertation, Göttingen (2001)

[28] S. H. Lee, M. A. McHugh, Polymer 38 Nr. 6 (1996) 1317 


\section{Modellierungen mit der PC-SAFT-Zustands- gleichung}

\subsection{Einleitung}

Die Zielsetzung der in dieser Arbeit ausgeführten Modellierungen besteht darin, die Eignung

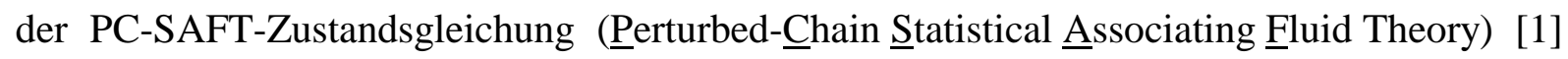
zur Beschreibung des Trübungsdruckverhaltens von Mischungen aus Ethen und Ethen(Meth)Acrylat-Copolymeren zu untersuchen. Im Folgenden wird das Konzept von PC-SAFT in knapper Form erläutert. Für eine detaillierte Darstellung der Methoden zur Berechnung von Phasengleichgewichten und der Entwicklung der verschiedenen hierzu angewandten $\mathrm{Zu}$ standsgleichung wird auf die Literatur $[2,3,4,11]$ verwiesen.

Die PC-SAFT-Zustandsgleichung stellt eine Modifikation der auf Chapman et al. [5] zurückgehenden SAFT-Zustandsgleichung dar. Die SAFT-Gleichung basiert auf der sogenannten Störungstheorie nach Barker und Henderson [6]. Die wesentliche Annahme besteht darin, dass der residuelle Anteil (Differenz zum Zustand „Ideales Gas“) der Freien Energie A $^{\text {res }}$ durch die Summe mehrerer Terme beschrieben werden kann, wobei der Hauptbeitrag durch die Freie Energie eines Referenzsystems $A^{\text {ref }}$ gegeben ist. Beiträge zur Freien Energie, welche nicht durch den Anteil des Referenzsystems gegeben sind, werden als Störungen betrachtet und in dem Ausdruck $\mathrm{A}^{\text {per }}$ (Perturbation) zusammengefasst.

Ein sehr häufig verwendetes Referenzsystem ist das „Harte-Kugel-Modell“. Hierbei werden die Moleküle als Kugeln mit festgelegtem Durchmesser und ohne anziehende Wechselwirkungen betrachtet. Ein derartiges Referenzsystem berücksichtigt die abstoßenden Wechselwirkungen, von welchen angenommen wird, dass sie die thermodynamischen Eigenschaften eines Systems maßgeblich bestimmen. Durch die zumeist nichtkugelförmige Gestalt realer Moleküle (insbesondere von Polymeren) und dem Auftreten von Dispersionsund Assoziationswechselwirkungen können Abweichungen vom Referenzsystem eintreten. Hierdurch bedingte Beiträge zur Freien Energie werden durch Störungsterme berücksichtigt. Im Rahmen der SAFT-Theorie wird angenommen, dass kettenartige Moleküle (Polymere und Lösungsmittel) aus Ketten identischer kugelförmiger Segmente bestehen. Ausgehend vom Referenzsystem „Harte Kugel“ berücksichtigt SAFT drei unabhängige Störungsbeiträge; Anziehende Wechselwirkungen nichtgebundener Segmente $\left(\mathrm{A}^{\text {disp }}\right)$, Kettenbildung harter Kugeln $\left(\mathrm{A}^{\text {chain }}\right)$ und assoziative Wechselwirkungen. 
Der neue Ansatz der PC-SAFT-Zustandsgleichung besteht darin anstelle der „Harten-Kugel“ das Referenzsystem „Harte-Kette“ zu verwenden. Der Dispersionsterm beinhaltet daher die anziehende Wechselwirkung von Kettenmolekülen anstelle von ungebundenen kugelförmigen Molekülen und ist abhängig von der Kettenlänge m. Die residuelle Freie Helmholtz-Energie berechnet sich somit zu:

$$
\mathrm{A}^{\text {res }}=\mathrm{A}^{\text {hard chain }}+\mathrm{A}^{\text {disp }}(\mathrm{m})+\mathrm{A}^{\text {assoc }}
$$

Die PC-SAFT-Zustandsgleichung sieht für jede Komponente drei Reinstoffparameter vor, welche jeweils eine physikalische Bedeutung aufweisen. Es handelt sich um zwei geometrische Parameter; den Segmentdurchmesser $\sigma$ und die Segmentzahl $m$ eines Moleküls und einen Energieparameter, $\varepsilon / k$ für die Wechselwirkung zwischen zwei Segmenten. Darüber hinaus wird ein binärer Wechselwirkungsparameter für jede Kombination zweier verschiedener Segmente definiert. Bedeutsam ist dabei, dass alle Reinstoffparameter als nicht temperaturabhängig erachtet werden.

Innerhalb des Modells können Copolymere als aus verschieden großen sphärischen Segmenten bestehende Polymerketten betrachtet werden. Die theoretischen Grundlagen zur Erweiterung des SAFT-Modells auf Copolymere wurde von Amos und Jackson [7], Banaszak et al. [8], und Shukla und Chapman [9] erarbeitet. In dieser Arbeit wird die Copolymervariante der PC-SAFT-Gleichung [11] verwendet. Bezüglich der Details der Ausdrücke zur Berechnung der Freien Helmholtz-Energie wird auf [11] verwiesen.

Die Abfolge der verschiedenen Segmente innerhalb eines Copolymermoleküls muss für die SAFT-Modellierung nicht exakt bekannt sein. Die Anzahl der binären Segment-Kontakte ist durch die beiden folgenden Größen definiert: Segmentverhältnis $z_{\mathrm{i} \alpha}$ und Bindungsverhältnis $B_{\mathrm{i} \alpha \mathrm{i} \beta}$. Das Segmentverhältnis berechnet sich nach: $z_{\mathrm{i} \alpha}=m_{\mathrm{i} \alpha} / m_{\mathrm{i}}$ wobei $m_{\mathrm{i} \alpha}$ die Anzahl der Segmente des Typs $\alpha$ in der Copolymerkette i darstellt, welche insgesamt aus $m_{\mathrm{i}}$ Segmenten zusammengesetzt ist. $m_{\mathrm{i} \alpha}$ ergibt sich aus: $m_{\mathrm{i} \alpha}=w_{\mathrm{i} \alpha} \cdot M_{\mathrm{i}} \cdot(m / M)_{\mathrm{i} \alpha}$. Hierbei bezeichnet $w_{\mathrm{i} \alpha}$ das Massenverhältnis von Monomer Typ $\alpha$ im Copolymermolekül. $M_{\mathrm{i}}$ ist das Molekulargewicht des Copolymer I und $(m / M)_{\text {i }}$ ist die Anzahl Segmente des Typs $\alpha$ bezogen auf die Masse des $\alpha$-Monomers. Das Bindungsverhältnis $B_{\mathrm{i} \alpha \mathrm{i} \beta}$ bezeichnet den Anteil der Bindungen zwischen den $\alpha$ - und $\beta$-Segmenten zur Gesamtzahl an Segment-Segment-Bindungen. 
Bei den in dieser Arbeit untersuchten Copolymere handelt es sich um Produkte mit bestmöglicher chemischer Einheitlichkeit (vgl. Abschnitt 3) und statistischer Verteilung der Segmentbausteine. Für die Modellierung werden nach [11] für statistische Copolymere des Typs Poly $(\alpha-c o-\beta)$ folgende Annahmen getroffen:

(I) wenn der Anteil der $\beta$-Segmente in der Polymerkette kleiner ist als der von $\alpha$ werden alle $\beta$-Segmente als jeweils ausschließlich mit benachbarten $\alpha$-Segmenten verknüpft erachtet. $\beta-\beta-$-Verknüpfungen werden folglich nicht berücksichtigt.

(II) Copolymere mit identischer Anzahl von $\alpha$ - and $\beta$-Segmenten werden als streng alternierend angenommen.

Die Bindungsverhältnisse für statistische Copolymere mit $z_{\mathrm{i} \beta}<z_{\mathrm{i \alpha}}$ berechnen sich nach:

$B_{\mathrm{i} \alpha \beta}=2 \cdot\left(m_{\mathrm{i} \beta} /\left(m_{\mathrm{i}}-1\right)\right)$

$B_{\mathrm{i} \beta \mathrm{i} \beta}=0$

$B_{\mathrm{i} \alpha \mathrm{i \alpha}}=1-B_{\mathrm{i} \alpha \beta}$

Zur Beschreibung eines nicht assoziierenden Copolymermoleküls (Index I) mit PC-SAFT werden für jeden Segmenttyp drei Reinstoffparameter (s.o.) benötigt. Diese sind, mit Index $\alpha$ für einen der beiden Segmenttypen; der Segmentdurchmesser $\sigma_{\mathrm{i} \alpha}$, die Segmentzahl $m_{\mathrm{i} \alpha}$ für Segment $\alpha$ im Copolymer und der Energieparameter $\varepsilon_{\mathrm{i} \alpha} / k$. Hinzu kommt der Parameter für die binäre Wechselwirkung zwischen Segment $\alpha$ und $\beta$.

Bei der Modellierung einer Mischung eines Lösungsmittels mit einem Copolymer wie z.B. Ethen / Ethen-Ethylacrylat-Copolymer braucht man somit die Reinstoffparameter von Polyethylen, von Poly(EA) und von Ethen. Darüber hinaus sind die binären Wechselwirkungsparamter $k_{\mathrm{i} \alpha \mathrm{i} \beta}, k_{\mathrm{i} \alpha \mathrm{E}}$, and $k_{\mathrm{i} \beta \mathrm{E}}$ erforderlich. $k_{\mathrm{i} \alpha \mathrm{E}}$ und $k_{\mathrm{i} \beta \mathrm{E}}$ repräsentieren die Wechselwirkung zwischen dem jeweiligen Homopolymer-Segment und Ethen (Solvens). Diese Parameter sollen in dieser Arbeit soweit möglich anhand von experimentellen Daten zu den Randsystemen Ethen / Hompolymer und damit unabhängig von den Copolymer-Ergebnissen ermittelt werden. Der Parameter $k_{\mathrm{i} \alpha \mathrm{i} \beta}$, welcher die Wechselwirkung der verschiedenen Segmente untereinander innerhalb des Copolymermoleküls beschreibt, ist somit der einzige der aus Anpassung an die Copolymerdaten erhalten wird. 


\subsection{Ergebnisse}

\subsubsection{System Ethen / Ethen-Methylacrylat-Copolymer}

Für die Modellierung von Ethen / Ethen-Copolymer-Systemen mit dem PC-SAFT-Modell werden, wie im vorherigen Abschnitt erläutert, die Reinstoffparameter für das Lösungsmittel Ethen und für beide zugehörigen Homopolymere benötigt. Für die Behandlung des Systems Ethen / Ethen-Methylacrylat-Copolymer ist somit die Kenntnis der Reinstoffparameter von Ethen, Polyethylen und von Polymethylacrylat (Poly(MA)) erforderlich. Die Parameter für Ethen und Polyethylen als auch der zugehörige binäre Parameter des Ethen-PolyethylenSystems $k_{\mathrm{ij}}$ sind aus der Literatur bekannt [10]. Für das System Ethen / Poly(MA) wie auch für das System Ethen / Poly(MMA) konnten experimentell keine Trübungsdrücke bestimmt werden, da diese Systeme offenbar so hohe Trübungsdrücke aufweisen, dass sie innerhalb der Grenzen der in dieser Arbeit verwendeten Messtechnik $\left(260^{\circ} \mathrm{C}, 3000\right.$ bar, vgl. Abschnitt 3.3.6) nicht homogen mischbar sind. Zur Umgehung dieses Problems wurden daher Trübungsdruckmessungen unter Anwendung des für Poly(meth)acrylate besseren Lösungsmittels Propen vorgenommen. Die Reinstoffparameter für Poly(MA) und Poly(MMA) können anschließend durch eine gleichzeitige Anpassung der Dichten von Poly((M)MA) [15] und der Trübungsdruckkurven für Propen / Poly((M)MA) ermittelt werden, wobei ausgenutzt wird, dass Reinstoffdaten für Propen bereits bekannt sind. Die erhaltenen Werte sind im Anhang D zusammen mit den Reinstoffparametern der weiteren in dieser Arbeit untersuchten Homopolymere aufgelistet.

Anzumerken ist, dass der hier ermittelte Energieparameter für Poly(MA) und der binäre Wechselwirkungsparameter für Propen / Poly(MA) sich von den von Groß et al. [11] bereits publizierten Werten unterscheiden. Allerdings beruhen die Literaturdaten, da bislang keine experimentellen Trübungsdrücke für Propen / Poly(MA) verfügbar waren, auf Anpassungen an Poly(MA)-Dichten und Ergebnissen zum System Octanon / Poly(MA) im Druckbereich von 20 bis 50 bar. Dies bedeutet, dass ein erheblich niedrigerer Druckbereich untersucht wurde und weiterhin ein polares Lösungsmittel und damit eines gänzlich anderen Typs als bei den Messungen in dieser Arbeit verwendet wurde. Darüberhinaus musste der binäre Parameter für Propen / PolyMA an Trübungsdrücke für das Coplymersystem Propen / E-MA angepasst werden. Mit den in [11] mitgeteilten Werten konnten die experimentellen Ergebnisse für Propen / Poly(MA) in dieser Arbeit nicht korrekt beschrieben werden. Die in dieser Arbeit ausgeführte erneute Bestimmung erscheint daher gerechtfertigt als auch bezüglich des Ergebnisses verlässlicher. 
In Abbildung 6.1 wird die Eignung der in dieser Arbeit erhaltenen Parameter zur Beschreibung der experimentellen Daten von Propen / Poly(MA) und Propen / Poly(MMA) mit dem PC-SAFT-Modell illustriert.

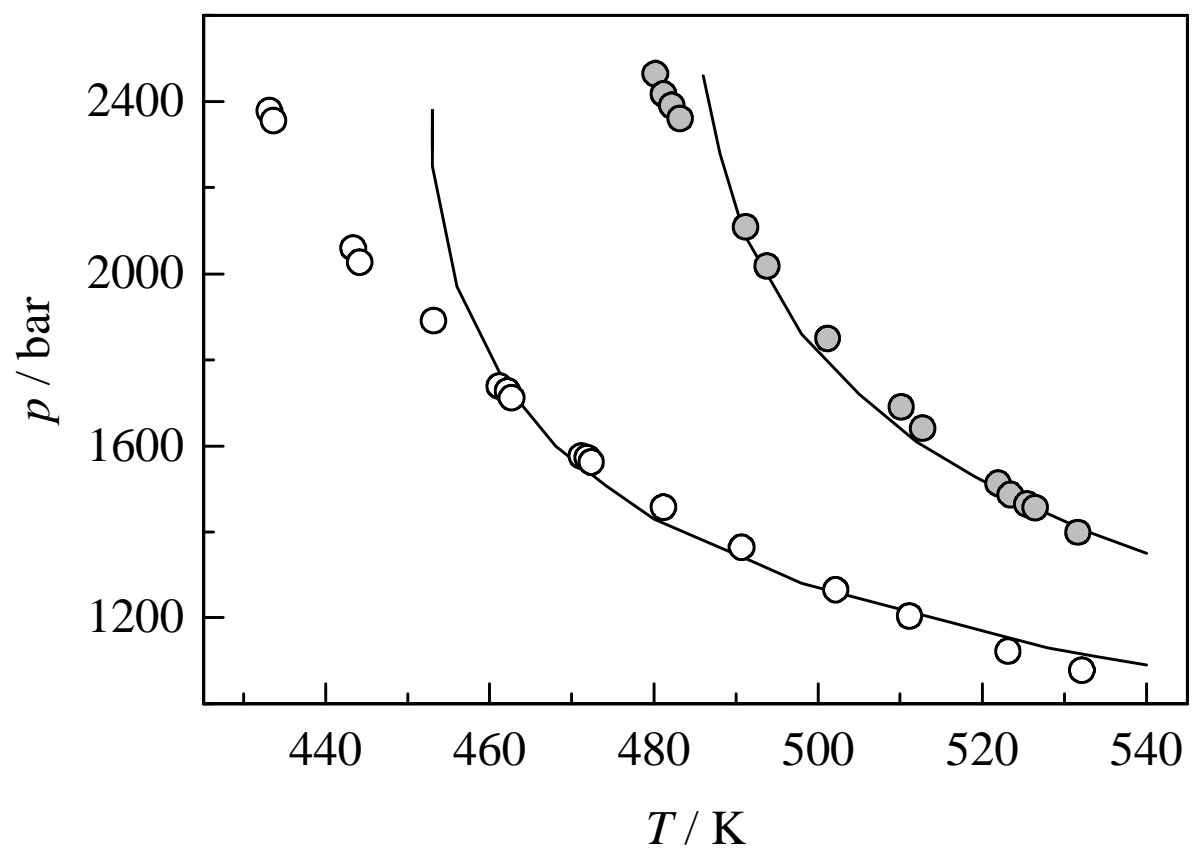

Abbildung 6.1: Trübungsdrücke für Mischungen aus Propen mit Poly(MA) bzw. Propen mit Poly(MMA). Die Symbole kennzeichnen die experimentellen Daten. Die Linien stellen die Ergebnisse mit den in Anhang D aufgelisteten Parametern durchgeführten Modellrechnungen mit PC-SAFT dar.

Bei der Beurteilung der Güte der Übereinstimmung von gerechneten und gemessenen Daten ist zu berücksichtigen, dass für die Modellierungen der experimentell bestimmte $M_{\mathrm{w}}$-Wert als Molekulargewicht des Polymers eingesetzt und somit die Polydispersität vernachlässigt wird. Mit Ausnahme des Druckbereiches oberhalb von 2200 bar bei Propen/Poly(MA) und oberhalb von 1800 bar bei Propen / Poly(MMA) stimmen die Ergebnisse der Modellierungen (Linien) gut mit den experimentellen Daten (Symbole) überein.

In Abbildung 6.2 werden Trübungskurven für Mischungen aus Ethen und Ethen-Methylacrylat-Copolymeren mit unterschiedlichem MA-Gehalt im Copolymer gezeigt. Die experimentellen Datenpunkte wurden einer vorangegangenen Arbeit [12] entnommen. Die Ergebnisse der unter Anwendung der in Anhang D aufgelisteten Parameter ausgeführten Modellierung mit PC-SAFT sind als Linien dargestellt. Zum Vergleich ist in Abbildung 6.2 außerdem eine Trübungskurve für Ethen / Polyethylen aufgenommen. 


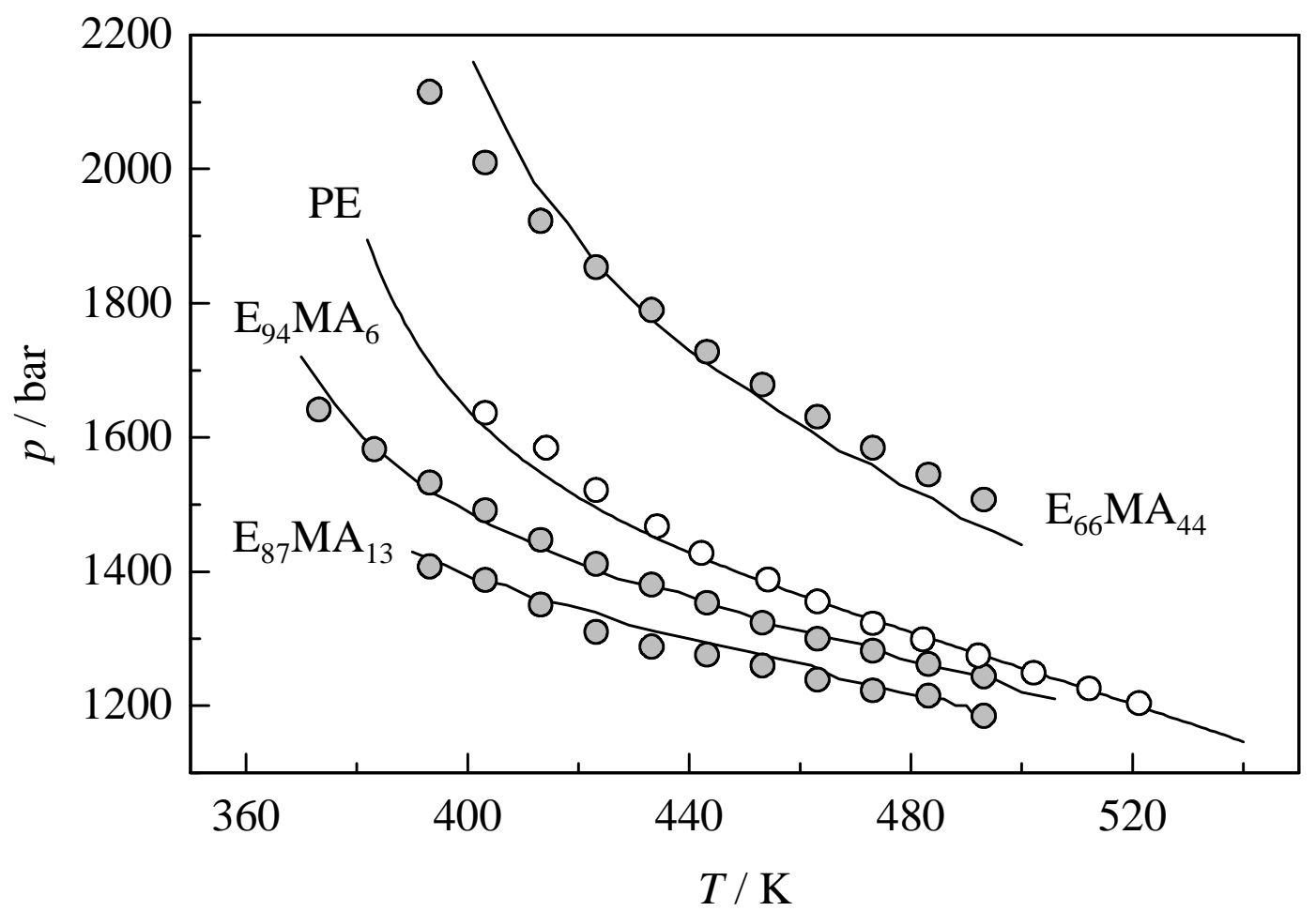

Abbildung 6.2: $\quad$ Trübungsdruckkurven für Mischungen aus Ethen und Ethen-MethylacrylatCopolymeren bei einer Polymerkonzentration von 5 Gew.\%. Die experimentellen Daten (Symbole) sind mit Ausnahme der Ergebnisse für Polyethylen der Literatur [12] entnommen. Die Linien entsprechen Modellrechnungen mit der PC-SAFT-Zustandsgleichung unter Anwendung der im Anhang D auf-geführten Parameter.

Ausgehend vom Entmischungsdruck für Polyethylen wird der Trübungsdruck mit steigenden MA-Gehalt im Copolymer zunächst abgesenkt. Wie bereits erwähnt, ist Poly(MA) bei einer Polymerkonzentration von 5 Gew.\% innerhalb der experimentellen Druck- und Temperaturgrenzen in Ethen unlöslich. Der nach dem PC-SAFT-Modell für eine Mischung aus Ethen und 5 Gew.\% Poly(MA) mit einem mittleren Molekulargewicht $\left(M_{\mathrm{w}}\right)$ von $100 \mathrm{~kg} / \mathrm{mol}$ bei $250^{\circ} \mathrm{C}$ zu erwartende Trübungsdruck beträgt ca. 3200 bar. Anhand der in Abbildung 6.2 für das Ethen / E-MA-System dargestellten experimentellen und modellierten Trübungsdrücke wird gezeigt, dass durch Anwendung eines einzigen an die Daten anzupassenden Parameters (binärer Parameter $k_{\mathrm{i} \alpha \mathrm{i} \beta}$, zur Beschreibung der Wechselwirkung zwischen Methylacrylatsegment und Ethylensegment) eine sehr gute Übereinstimmung erreicht werden kann. Die Anpassung ergibt eine in guter Näherung lineare Abhängigkeit von $k_{\mathrm{i} \alpha \mathrm{i} \beta}$ vom MA-Gehalt im Copolymer (siehe Abbildung 6.3). Der vollständige Parametersatz, der bei den Modellrechnungen für Ethen/E-MA und die weiteren in dieser Arbeit untersuchten Copolymersysteme verwendet wurde ist im Anhang D angegeben. 


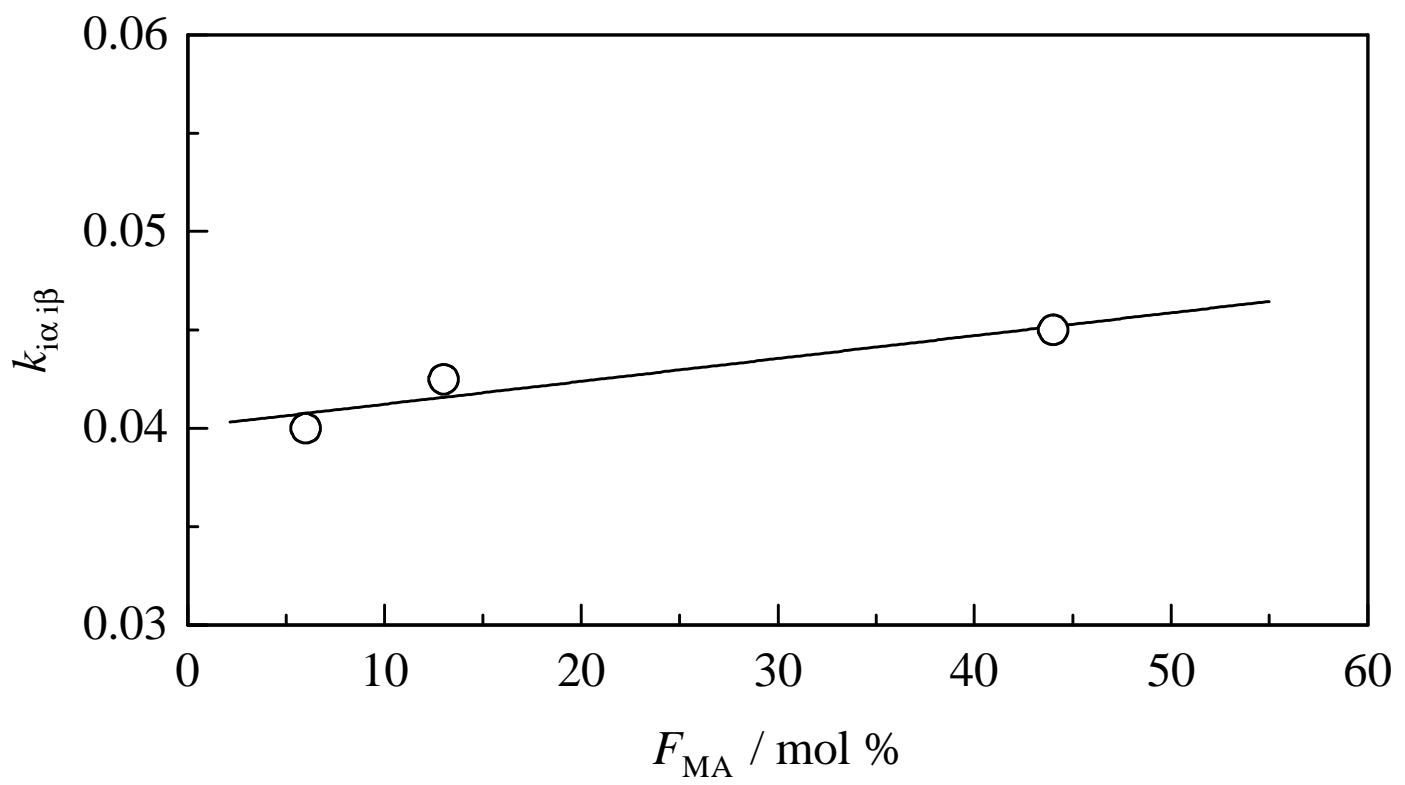

Abbildung 6.3: Abhängigkeit des bei der Modellierung des Systems Ethen/E-MA verwendeten binären Wechselwirkungsparameter $k_{i \alpha i \beta}$ vom MA-Gehalt im Copolymer.

Die Abhängigkeit des Trübungsdrucks von der Copolymerzusammensetzung $F_{\mathrm{MA}}$ wird von Byun et al. [13] mit der Wirkung zweier gegenläufiger Effekte erklärt. Bei kleinen $F_{\mathrm{MA}^{-}}$ Werten verbessern die mit dem Einbau der polaren MA-Einheiten ins Copolymer möglichen Wechselwirkungen des Quadrupolmoments des Lösungmittels Ethen mit den MA-Einheiten die Löslichkeit. Mit zunehmenden MA-Gehalt im Copolymer erhalten verstärkt intermolekulare polare Wechselwirkungen zwischen den Carboxylgruppen der MA-Einheiten im Copolymer Bedeutung, welche eine Verschlechterung der Löslichkeit bewirken. Diese Wechselwirkung sollte oberhalb von $F_{\mathrm{MA}} \approx 20 \mathrm{~mol} \%$ dominant werden und das Löslichkeitsverhalten bestimmen. Die bei geringen $F_{\mathrm{MA}}$-Werten auftretende Erniedrigung des Trübungsdrucks mit $F_{\mathrm{MA}}$ kann allerdings auch mit der Wirkung der eingebauten MA-Einheiten als Kurzkettenverzweigungen erklärt werden. So ist aus Studien zum Entmischungsverhalten von Ethen- $\alpha$-Olefin-Copolymeren bekannt, dass sich die Copolymerlöslichkeit in Ethen mit zunehmendem Anteil Kurzkettenverzweigungen verbessert [14]. 


\subsubsection{System Ethen / Ethen-Ethylacrylat-Copolymer}

In Abbildung 6.4 sind die für das System Ethen/E-EA und die Randsysteme Ethen / Polyethylen bzw. Ethen / Poly(EA) gemessenen Trübungsdrücke gemeinsam mit den Ergebnissen von PC-SAFT-Modellierungen dargestellt.

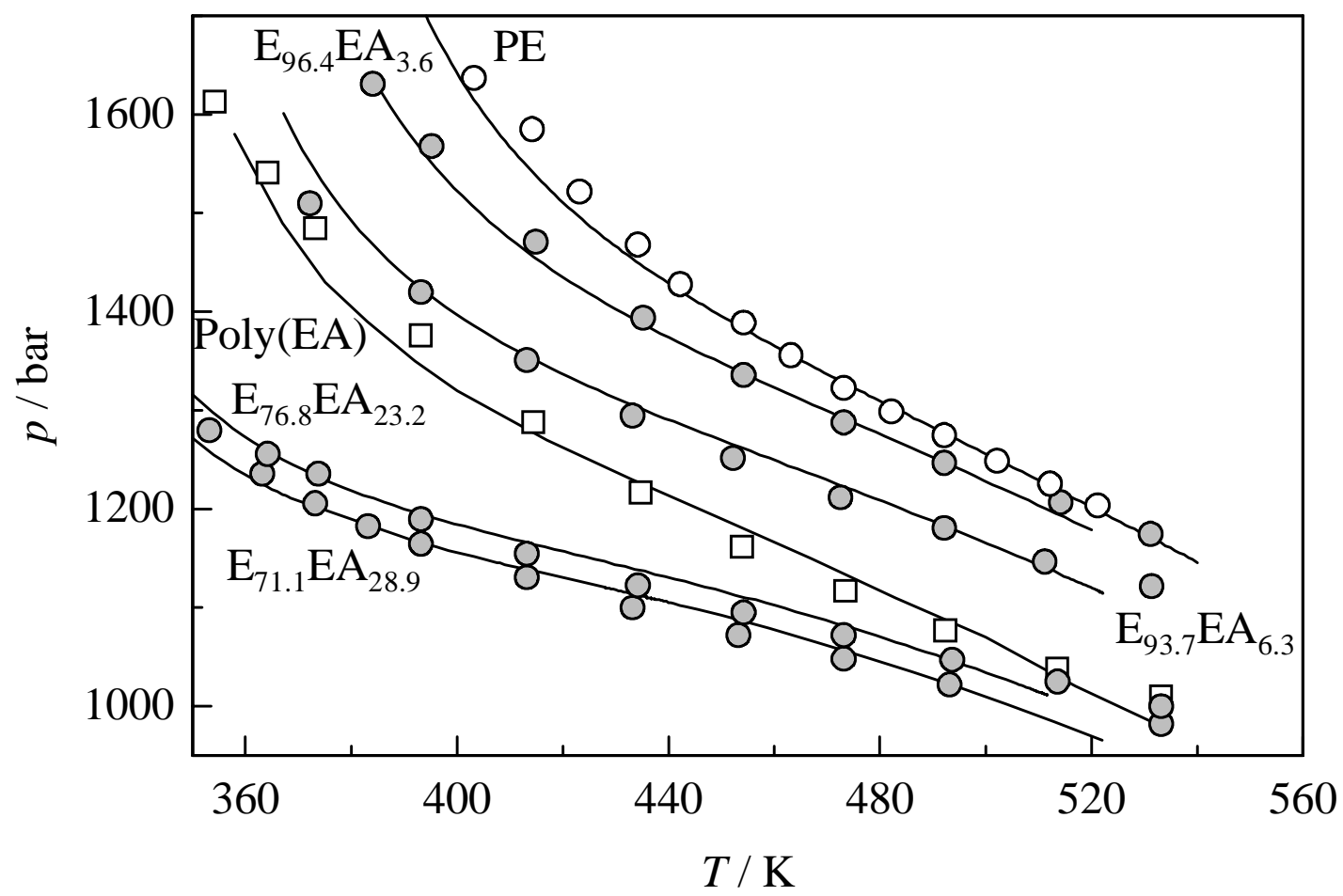

Abbildung 6.4: Trübungsdruckkurven für Mischungen aus Ethen und Ethen-EthylacrylatCopolymeren mit unterschiedlichem Ethylacrylatgehalt im Copolymer. Die Symbole stehen für experimentelle Datenpunkte. Die Linien entsprechen Modellrechnungen mit der PC-SAFT-Zustandsgleichung.

Die Resultate der Modellierungen sind als durchgezogene Linien und die experimentellen Daten als Symbole gezeichnet. Ähnlich wie bei der Situation für Ethen / E-MA nimmt der Trübungsdruck für Ethen / E-EA von ethenreichen Copolymeren mit zunehmenden EAGehalt im Copolymer zunächst ab. Nach Durchlaufen eines Minimums bei einem EA-Gehalt von ca. 30 mol-\% steigt der Trübungsdruck wieder an. Die Reinstoffparameter für Poly(EA) wurden durch gleichzeitiges Anpassen an Dichtedaten für Poly(EA) [15] und an die Trübungsdrücke für E/Poly(EA) bestimmt. Für den binären Parameter $k_{\mathrm{ij}}$, welcher die Wechselwirkungen zwischen Ethen und dem Homopolymer Poly(EA) beschreibt, wurde ein Wert von 0.0135 erhalten.. Aus Abbildung 6.4 ist die sehr gute Übereinstimmung der mit PCSAFT berechneten Trübungsdrücke und der experimentellen Daten klar erkennbar. Für den Parameter $k_{\mathrm{i} \alpha \mathrm{i} \beta}$, welcher die Wechselwirkung zwischen polymeren Ethylensegmenten und 
Ethylacrylatsegmenten berücksichtigt, wurde durch Anpassung an die experimentellen Daten ein (konstanter) Wert von 0.020 bestimmt. Für das Ethen / E-EA System ergibt sich daher die besonders vorteilhafte Situation, dass das Trübungsdruckverhalten im untersuchten Zustandsund Zusammensetzungsbereich ausschließlich mit Reinstoffparametern und drei binären, weder von der Temperatur noch von der Copolymerzusammensetzung abhängenden, Parametern beschrieben werden kann.

\subsubsection{System Ethen / Ethen-Propylacrylat-Copolymer}

Die für das System Ethen / E-PA bestimmten Trübungsdrücke finden sich in Abbildung 6.5. Im Unterschied zu den Beobachtungen für die homologen Systeme E / E-MA und E / E-EA nimmt der Trübungsdruck für E / E-PA ausgehend von Ethen / Polyethylen bis zum zweiten Randsystem Ethen / Poly(PA) stetig ab.

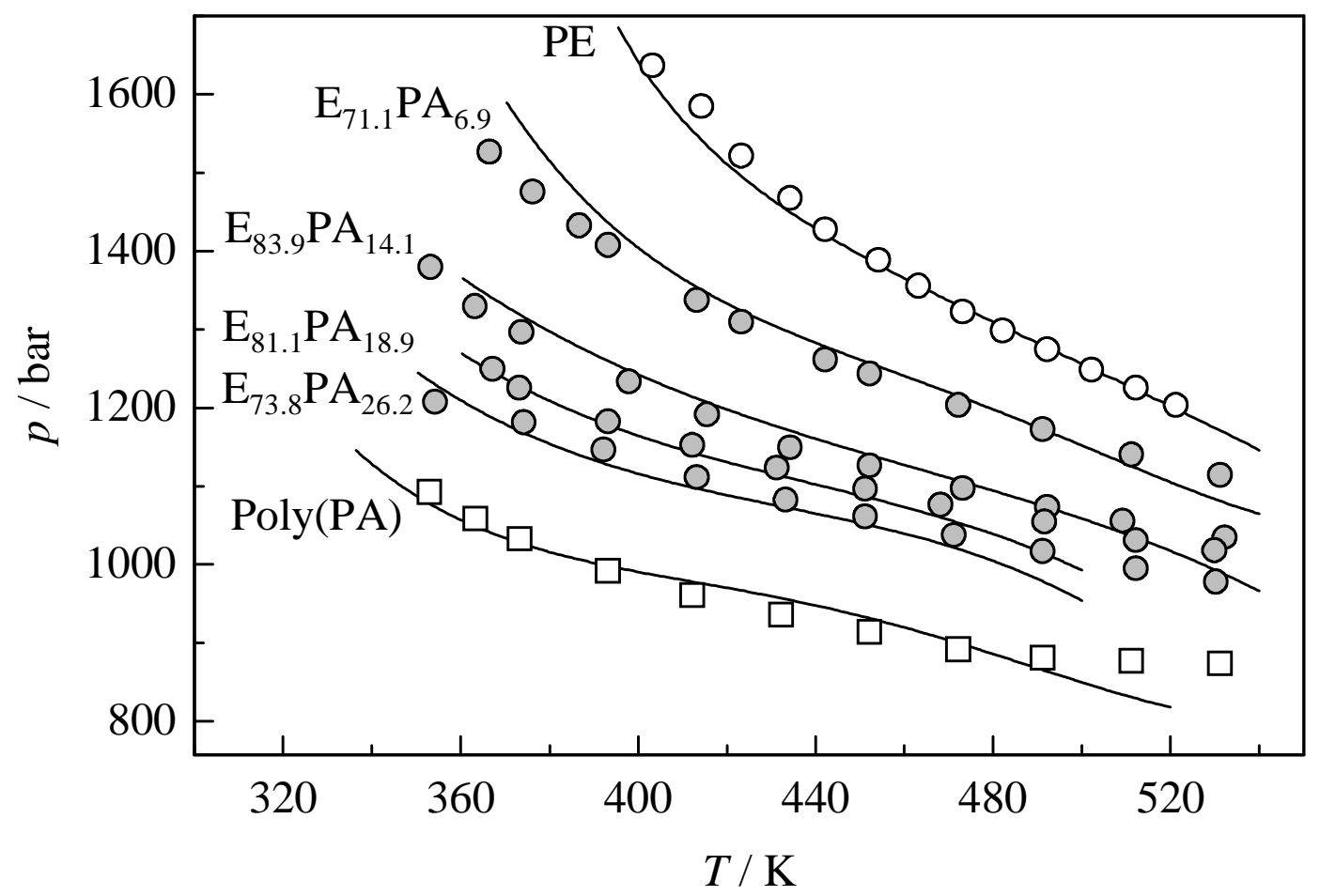

Abbildung 6.5: Trübungsdruckkurven für Mischungen aus Ethen und Ethen-PropylacrylatCopolymeren mit unterschiedlichem Propylacrylatgehalt im Copolymer. Die Symbole stellen experimentelle Datenpunkte dar. Die Ergebnisse der Modellrechnungen mit der PC-SAFT-Zustandsgleichung sind als Linien gezeichnet.

Für das System Ethen / E-PA musste eine alternative Strategie zur Bestimmung der Poly(PA)Reinstoffparameter verwendet werden, da bislang keine Dichtedaten für Poly(PA) aus der Literatur bekannt sind. Aus diesem Grund wurden die Reinstoffparameter für Poly(PA) aus jeweils einer Auftragung der Werte der Parameter für Poly(MA), Poly(EA) und Poly(BA) 
gegen die Zahl der Kohlenstoffatome der Alkylestergruppe durch Interpolation ermittelt. Die Vorgehensweise wird in Abbildung 6.6 illustriert.

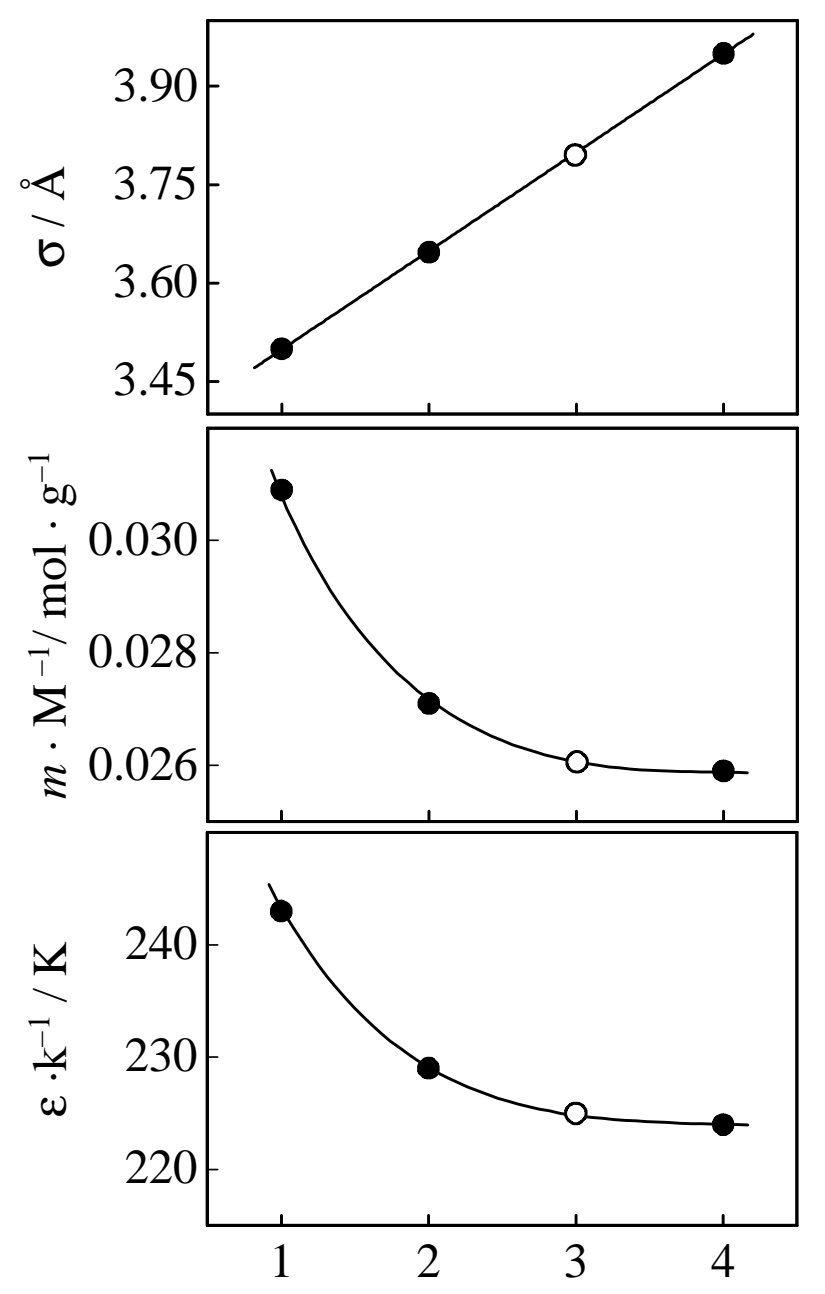

Anzahl Kohlenstoffatome
Abbildung 6.6:

Auftragung der Reinstoffparameter: Quotient aus Segmentzahl und Molekulargewicht $m / M$, Energieparameter $\varepsilon / k$ und Segmentdurchmesser $\sigma$ für Poly(MA), Poly(EA) und Poly(BA) gegen die Anzahl Kohlenstoffatome in der Alkylestergruppe. Die offenen Symbole stellen die Ergebnisse der Interpolation für Poly(PA) dar.

In der Grafik sind die Werte für $m / M$, $\varepsilon / k$ und $\sigma$ für die drei Homopolymere in Form gefüllter Symbole aufgetragen. Die offen gezeichneten Symbole entsprechen hierbei den interpolierten Werten für Poly(PA). Für alle drei Parameter wird innerhalb der homologen Reihe eine gute Korrelation mit der Anzahl der Kohlenstoffatome gefunden. Dies spricht für die Eignung der gewählten, eher pragmatischen Methode der Interpolation. Wie aus Abbildung 6.5 erkennbar können mit der PC-SAFT-Zustandsgleichung (durchgezogene Linien) unter Anwendung der durch Interpolation erhaltenen Parameter die experimentellen Trübungsdrücke (Symbole) auch für das System E / E-PA in guter Übereinstimmung beschrieben werden. 


\subsubsection{System Ethen / Ethen-Butylacrylat-Copolymer}

In Abbildung 6.7 werden die Ergebnisse der PC-SAFT-Modellierungen für das System Ethen / E-BA zusammen mit experimentellen Daten aus der Literatur [3,9] und einer in dieser Arbeit bestimmten Trübungsdruckkurve $\left(\mathrm{E}_{73.2} \mathrm{BA}_{26.8}\right)$ gezeigt. Vergleichbar zu den Beobachtungen für Ethen / E-PA, nimmt der Trübungsdruck bei konstanter Temperatur mit zunehmendem BA-Gehalt ab.

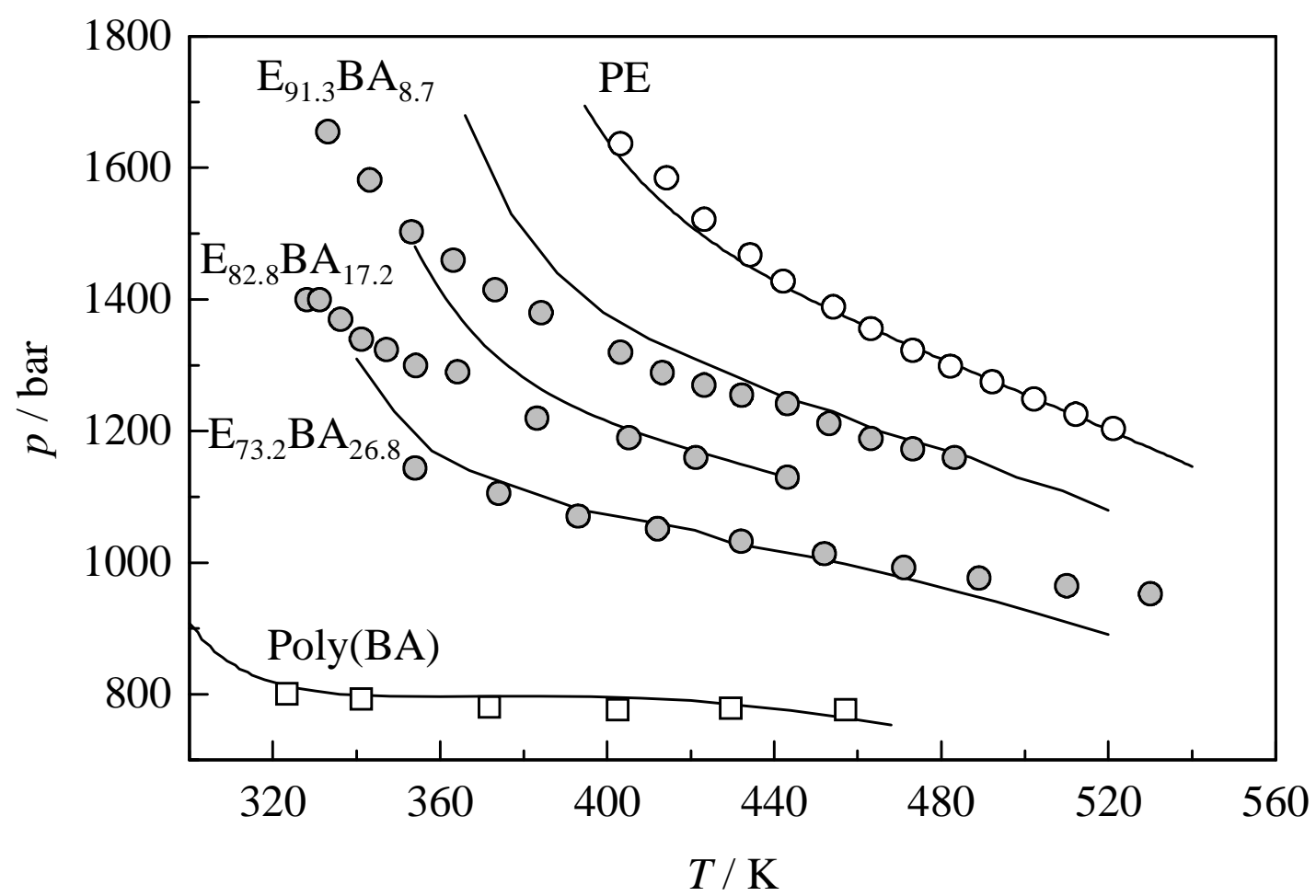

Abbildung 6.7: Trübungsdruckkurven für Mischungen aus Ethen und Ethen-ButylacrylatCopolymeren mit unterschiedlichem Butylacrylatgehalt im Copolymer. Die Symbole stehen für experimentelle Datenpunkte. Die Linien entsprechen Modellrechnungen mit der PC-SAFT-Zustandsgleichung.

Analog zu der Vorgehensweise für Ethen/E-EA wurden die Reinstoffparameter für Poly(BA) durch gleichzeitiges Anpassen an Dichten von Poly(BA) und Trübungsdruckdaten für Ethen / Poly(BA) ermittelt. Der binäre Wechselwirkungsparameter von Ethylen- und BASegmenten im Copolymer $k_{\mathrm{i} \alpha \mathrm{i} \beta}$, wurde hierbei zu Null bestimmt. Dies bedeutet, da $k_{\mathrm{i} \alpha \mathrm{i} \beta}$ praktisch einen Korrekturparameter darstellt, dass die Wechselwirkung für Ethylen- und BASegmente für das System Ethen / E-BA angemessen durch die Parameter der HomopolymerSysteme charakterisiert wird. Mit diesem besonders einfachen Modell wird, wie aus Abbildung 6.7 ersichtlich, eine zwar nicht perfekte, zumindest aber befriedigende Beschreibung der experimentellen Trübungsdrücke erreicht. 


\section{Diskussion des Einflusses der Länge des Esteralkylgruppe auf den Trübungsdruck}

In Abbildung 6.8 wird die Abhängigkeit des Trübungsdrucks vom Acrylatanteil $F_{\mathrm{A}}$ im Copolymer für die vollständige Serie der experimentell und modellierend untersuchten Ethen / E-Alkylacrylat-Systeme für $200^{\circ} \mathrm{C}$ dargestellt. Die durchgezogenen Kurven entsprechen den mit den angegebenen durchschnittlichen Molmassen erhaltenen Modellierungsergebnissen ${ }^{1}$. Die Symbole stehen für die experimentellen Daten.

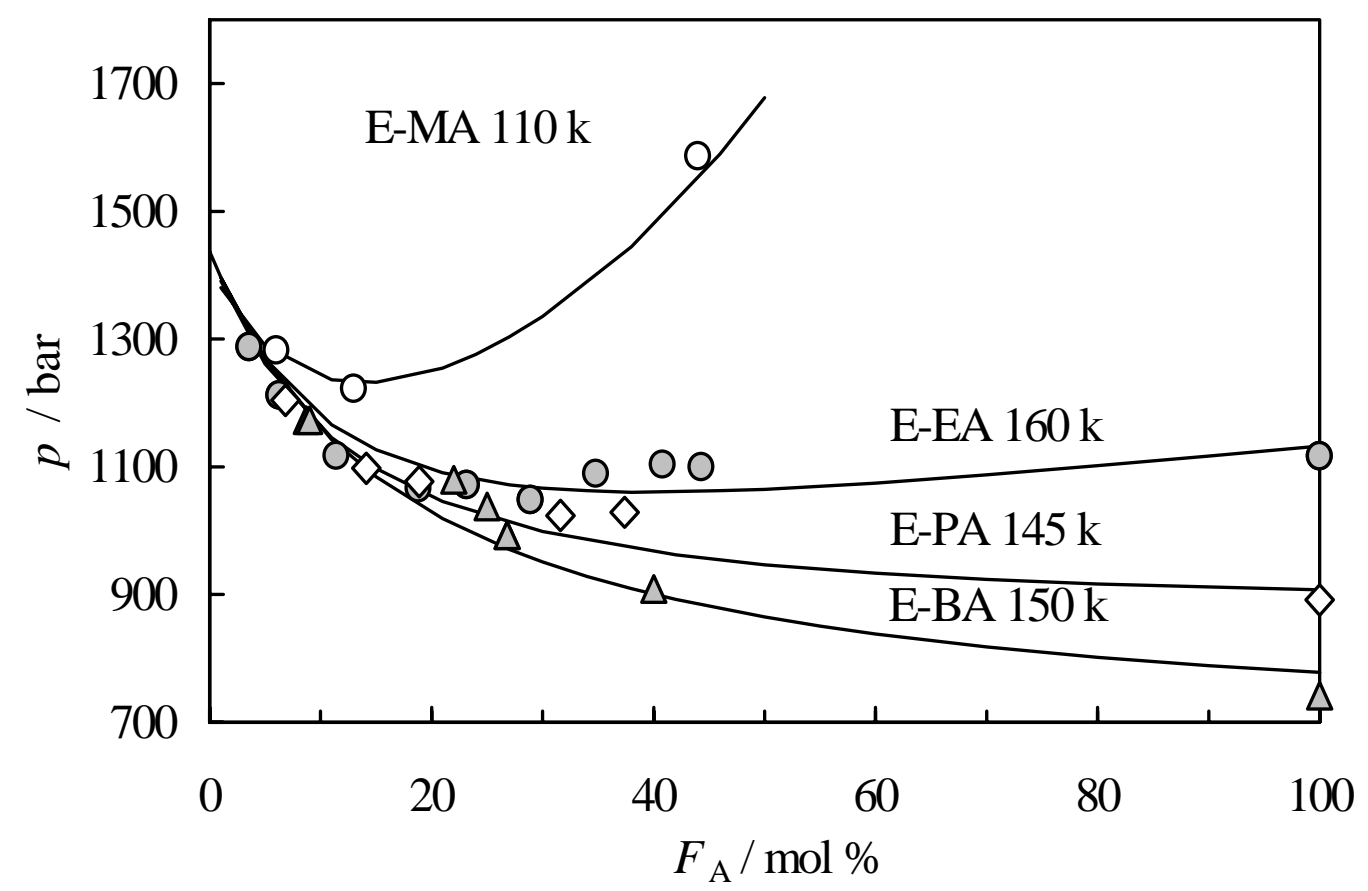

Abbildung 6.8: Abhängigkeit des Trübungsdrucks von E-MA-, E-EA-, E-PA- und E-BACopolymeren von der Copolymerzusammensetzung bei $200^{\circ} \mathrm{C}$ und einer Polymerkonzentration von 5 Gew.\% in Ethen. Die Symbole entsprechen Messdaten. Die Linien stehen für mit PC-SAFT berechnete Werte.

Folgende Beobachtungen ergeben sich aus Abbildung 6.8:

(1) Bei geringem Acrylatgehalt im Copolymer $\left(F_{\mathrm{A}}<10 \mathrm{~mol} \%\right)$ unterscheiden sich die Trübungsdrücke der verschiedenen Copolymersysteme kaum. Die Größe der Estergruppe spielt hier keine wesentliche Rolle.

(2) $\mathrm{Ab}$ einem Acrylatgehalt von ca. $10 \mathrm{~mol} \%$ differieren die Trübungsdrücke der Ethen / Ethen-Acrylat-Systeme. Für E-MA und E-EA durchläuft der Trübungsdruck mit zunehmenden Acrylatgehalt ein Minimum und steigt zu höheren $F_{\mathrm{A}^{-}}$ Werten an. Für E-MA wird ein ausgeprägtes, für E-EA nur ein flaches Minimum

\footnotetext{
${ }^{1}$ Die zum Teil weniger gute Deckung der Modellierungsergebnisse mit den experimentellen Daten in Abb. 6.8 beruht darauf, dass im Unterschied zu den Rechnungen zu den Trübungskurven bei den Modellierungen für Abb. 6.8 lediglich Mittelwerte der jeweiligen Molekulargewichte der Probenserien verwendet wurden.
} 
festgestellt. Für E-PA und E-BA nimmt der Trübungsdruck ausgehend von Polyethylen kontinuierlich bis zum entsprechenden Homopolymer Poly(PA) bzw. Poly(BA) ab.

(3) Unter der Annahme, dass das Ansteigen des Trübungsdrucks mit zunehmenden Acrylatgehalt durch intersegmentelle Wechselwirkungen der polaren Carboxylgruppen bedingt wird, zeigen die in Abbildung 6.8 dargestellten Ergebnisse, dass die Abschirmung der Carbonylfunktion durch lediglich eine Methylgruppe gering ist. Der deutliche Unterschied im Trübungsdruckverhalten von Ethen / E-MA und Ethen /E-EA zeigt auf, dass der Ersatz der Estermethylgruppe durch eine Ethylgruppe zu einer erheblich verstärkten Abschirmung führt. Die weitere Vergrößerung der Alkylestergruppe über die Propyl- zur Butylestergruppe verringert den Trübungsdruck nur noch in geringem Ausmaß. Übereinstimmend hierzu haben Studien zum Trübungsdruckverhalten des Systems Ethen / E-Ethylhexylacrylat [16] aufzeigen können, dass eine noch weitergehende Vergrößerung der Esteralkyleinheit den Trübungsdruck erwartungsgemäß im Vergleich nur wenig weiter absenkt. 


\subsubsection{System Ethen / Ethen-Methylmethacrylat-Copolymer}

Die für das System Ethen / E-MMA erhaltenen Modellierungsergebnisse sind in Abbildung 6.9 als durchgezogene Linien zusammen mit den experimentellen Daten (Symbole) dargestellt.

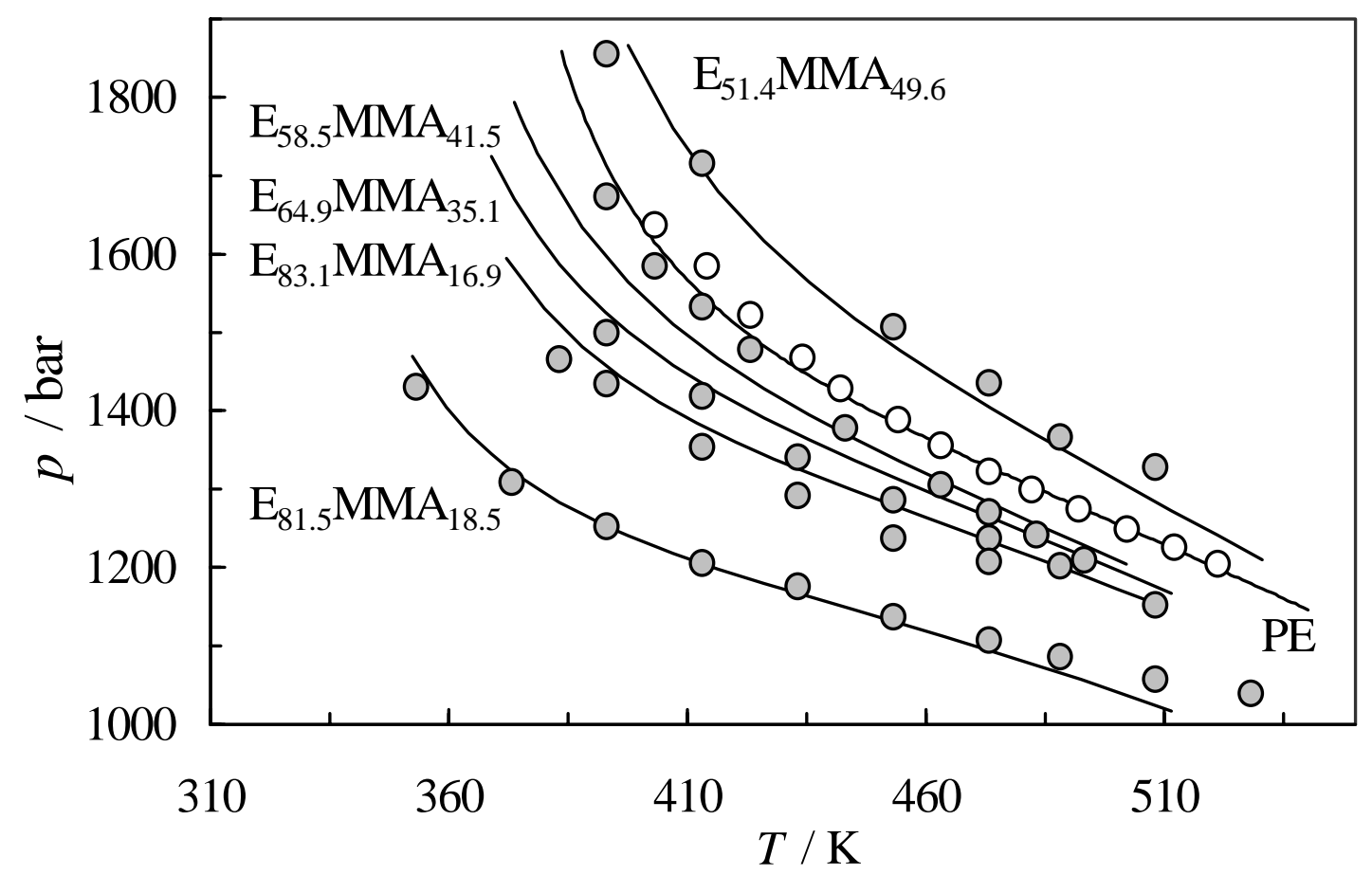

Abbildung 6.9: Trübungsdruckkurven für Mischungen aus Ethen und EthenMethylmethylacrylat-Copolymeren mit unterschiedlichem Methylmethacrylatgehalt im Copolymer. Die Symbole stellen experimentelle Datenpunkte dar. Die Linien entsprechen den Resultaten von Modellrechnungen mit der PC-SAFT-Zustandsgleichung.

Für Poly(MMA) wurden die Reinstoffparameter durch Anpassen der Propen / Poly(MMA)Trübungsdrücke analog zu der für Poly(MA) genutzten Vorgehensweise ermittelt (vgl. Abbildung 6.1). Die binären Parameter für Ethen / Poly(MMA) und die binären Parameter für die Wechselwirkung zwischen Ethylen- and Methylmethacrylat-Segmenten, $k_{\mathrm{i} \alpha \mathrm{i} \beta}$, wurden durch Anpassung an Trübungsdruckdaten für Ethen / E-MMA ermittelt. Hierbei zeigte sich, dass die experimentellen Daten gut beschrieben werden, wenn ähnlich zu Ethen / E-MA eine lineare Abhängigkeit von $k_{\mathrm{i} \alpha \mathrm{i} \beta}$ von der Copolymerzusammensetzung angenommen wird 


\subsubsection{System Ethen / Ethen-Butylmethacrylat-Copolymer}

In Abbildung 6.10 finden sich die für Ethen/E-BMA gemessenen Trübungsdrücke gemeinsam mit Ergebnissen der Modellrechnungen mit PC-SAFT. Vergleichbar mit dem System Ethen / E-MMA durchläuft der Trübungsdruck ein Minimum, welches allerdings erst bei einem relativ hohen Methacrylatgehalt von ca. $F_{\mathrm{BMA}}=44 \mathrm{~mol} \%$ auftritt. Das zugehörige Homopolymer Poly(BMA) ist in Relation zu Polyethylen bei deutlich geringeren Drücken in fluidem Ethen löslich.

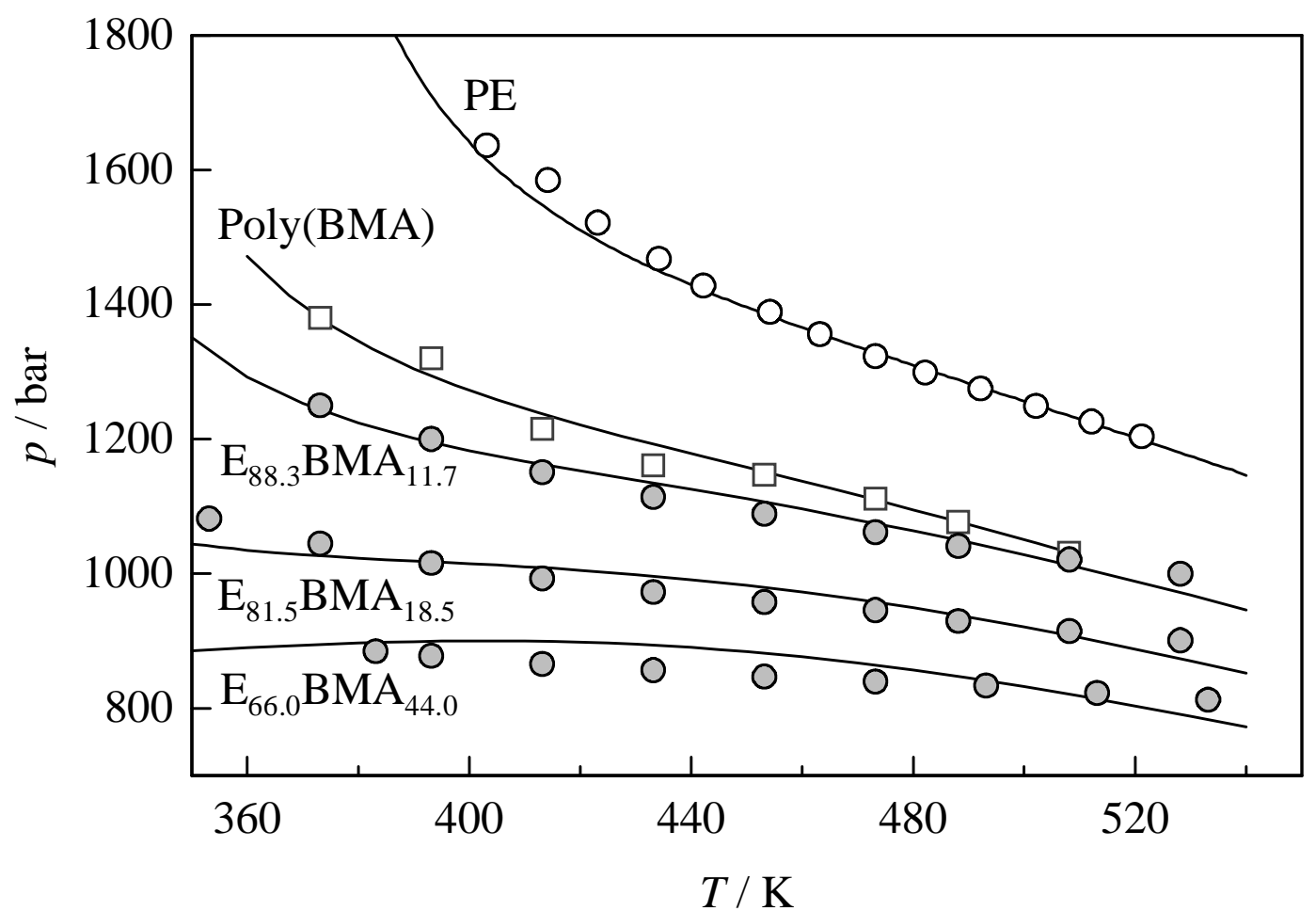

Abbildung 6.10: Trübungsdruckkurven für Mischungen aus Ethen und Ethen-Butylmethacrylat-Copolymeren mit unterschiedlichem Butylmethacrylatgehalt im Copolymer. Die Symbole stehen für experimentelle Datenpunkte. Die Linien entsprechen den Ergebnissen von Modellrechnungen mit der PCSAFT-Zustandsgleichung.

Die PC-SAFT-Modellierungen ergeben wie beim System Ethen/E-MMA eine gute Übereinstimmung der gerechneten mit den experimentellen Trübungsdrücken bei Berücksichtigung einer linearen Abhängigkeit des die Wechselwirkung von Ethylen- und Butylmethacrylat-Segmenten beschreibenden Parameters $k_{\mathrm{i \alpha} i \beta}$ mit dem BMA-Gehalt im Copolymer. 


\section{Diskussion des Einflusses der $\alpha$-Methylgruppe auf den Trübungsdruck}

Für einen Vergleich der Ethen / E-Methacrylat- mit den Ethen / E-Acrylat-Systemen mit identischer Alkylestergruppe ist in Abbildung 6.11 die Abhängigkeit des Trübungsdrucks von der Copolymerzusammensetzung $F_{\mathrm{A}}$ für Ethen/E-MA und Ethen/E-MMA sowie für Ethen / E-BA und Ethen / E-BMA für $200^{\circ} \mathrm{C}$ aufgetragen. Die experimentellen Daten gelten für Copolymere mit Molekulargewichten von 40 bis $130 \mathrm{~kg} / \mathrm{mol}$.

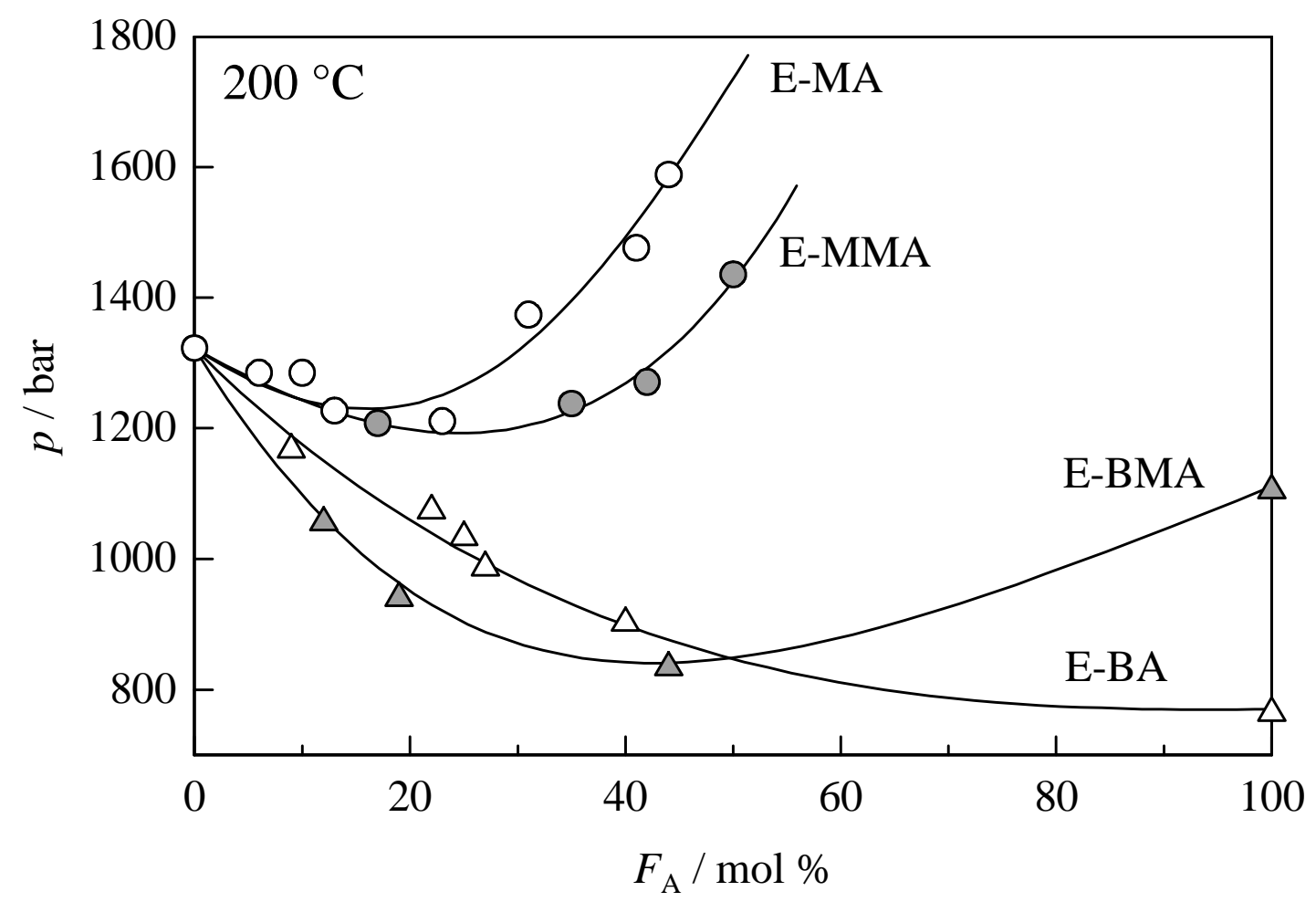

Abbildung 6.11: Abhängigkeit des Trübungsdrucks von der Copolymerzusammensetzung, $\left(F_{\mathrm{A}}\right)$ für E-MA und E-BA-Copolymere im Vergleich zu E-MMA- und EBMA-Copolymere bei $200^{\circ} \mathrm{C}$.

Das Absinken der Trübungsdrücke mit zunehmenden $F_{\mathrm{A}}$ ist für ethenreiche Methyl- und Butyl(meth)acrylat-Copolymere bis zu einer Copolymerzusammensetzung von $F_{\mathrm{A}}=6 \mathrm{~mol} \%$ jeweils ähnlich. Für die Copolymere mit Methylestergruppen wird bis etwa 20 mol \% ein nahezu übereinstimmender Verlauf der Trübungsdrücke beobachtet. $\mathrm{Zu}$ höheren $F_{\mathrm{A}}$-Werten ist der Wiederanstieg des Trübungsdrucks für Ethen / E-MA im Vergleich zu Ethen / E-MMA stärker ausgeprägt. Wenn angenommen wird, dass das Trübungsdruckverhalten von Ethen(Meth)Acrylat-Copolymeren maßgeblich von der Qualität der Abschirmung der polaren Carboxylatfunktionen durch die Esteralkylgruppen bestimmt wird, kann der Unterschied zwischen Ethen / E-MMA und Ethen / E-MA mit der zusätzlich abschirmenden Wirkung der 
$\alpha$-Methylgruppe des MMA-Comonomers erklärt werden. Dieser Ansatz macht allerdings nicht den Unterschied der Trübungsdrücke der E-BA- und E-BMA-enthaltenden Systeme verständlich, da bei diesen zwar im Bereich geringer B(M)A-Gehalte im Copolymer ebenfalls eine Löslichkeitsverbesserung durch die Anwesenheit von $\alpha$-Methylgruppe gefunden wird, allerdings bei höheren Gehalten der umgekehrte Effekt auftritt. Es ist daher zu vermuten, dass das Trübunsgdruckverhalten dieser Copolymersysteme durch weitere Effekte beeinflusst wird.

Der zusätzliche Einbau einer $\alpha$-Methylgruppe in die Hauptkette des Polymeren beim Übergang vom Comonomer MA zu MMA bzw. von BA zu BMA sollte außer zu einer verstärkten Abschirmung und damit zu verbesserter Löslichkeit auch zu einer verminderten Flexibilität der Polymerkette führen. Dieser in Richtung verschlechterter Löslichkeit und damit auf höhere Trübungsdrücke zielende Effekt ist verbunden mit dem Begriff der sogenannten "entropy penalty" [17]. Mit der Anwesenheit von $\alpha$-Methylgruppen in EthenCopolymeren sollten somit zwei auf den Trübungsdruck gegenläufig wirkende Effekte verknüpft sein. Es ist denkbar, dass die relative Bedeutung dieser beiden Effekte von der Größe der Alkylestergruppe abhängt. Es kann angenommen werden, dass bei den Systemen Ethen / E-MA und Ethen / E-MMA die zusätzliche Abschirmung durch die $\alpha$-Methylgruppe dominant ist.

Die Verringerung des Trübungsdrucks durch den Einbau von (Meth)Acrylat-Comonomeren bei geringem Comonomergehalt im Copolymer kann auch mit der Wirkung der Comonomergruppe als Kurzkettenverzweigung erklärt werden. Diese Annahme macht gleichzeitig die im angesprochenen Bereich geringeren Trübungsdrücke von Ethen/E-BMA im Vergleich zu Ethen / E-BA verständlich. Da intersegmentelle Wechselwirkungen der Carbonylgruppe durch die gute Abschirmung seitens der Butylestereinheiten bei den E-B(M)A-Copolymeren vermutlich nur eine untergeordnete Rolle spielen, sollte der “entropy-penalty”-Effekt für diese Copolymerfamilie bei hohem $F_{\mathrm{A}}$ besonders wirksam sein. Dies erklärt die bei höheren $F_{\mathrm{A}^{-}}$ Werten für E-BMA-System im Vergleich zu E-BA höheren Trübungsdrücke. Die im Vorangegangenen vorgestellte Annahme zweier gegenläufig wirkender Effekte (Verzweigungswirkung und "entropy penalty") durch den Einbau von Methacrylatcomonomeren stellt eine konsistente Erklärung des für Ethen/E-BMA und Ethen/E-BA beschriebenen „Kreuzens“ des Verlaufs des Trübungsdrucks mit $F_{\mathrm{A}}$ dar.

Es sei angemerkt, dass nicht ausgeschlossen werden kann, dass der signifikante Unterschied des Trübungsdrucks dieser beiden System bei hohem $F_{\mathrm{A}}$ möglicherweise auch aus 
unterschiedlichen Verzweigungsgraden von Kurzkettenverzweigungen resultiert. So ist für (Co)Polymerisationen [18] von Acrylaten bei hohen Temperaturen bekannt, dass das $\alpha$ Wasserstoffatom an der Polymerhauptkette eine hohe Transferaktivität aufweist. Somit könnte analog der Erklärung für die Absenkung des Trübungsdrucks bei geringem $F_{\mathrm{A}}$, die Trübungsdruckreduktion bei hohen BA-Gehalten im Copolymer durch das verstärkte Auftreten von Verzweigungen bewirkt werden. Für die Ethen / E-Methacrylat-Systeme ist aufgrund der Abwesenheit reaktiver $\alpha$-Wasserstoffatome, kein wesentlicher Anteil Verzweigungen zu erwarten. Für eine weitergehende Diskussion der Auswirkung von Verzweigungen auf das Entmischungsverhalten von Ethen-Copolymeren wird an dieser Stelle vorgeschlagen, eine Analyse der Polymermikrostruktur mittels quantitativer ${ }^{13} \mathrm{C}-\mathrm{NMR}$ auszuführen. 


\section{Diskussion des binären Wechselwirkungsparameters $k_{\mathrm{i} \alpha \mathrm{i} \beta}$}

Die im Rahmen dieser Arbeit durch Modellierung experimenteller Trübungsdruckdaten für die Systeme Ethen / E-(Meth)acrylat mit dem PC-SAFT-Modells erhaltenen Werte für $k_{\text {ioi }}$ sind angepasste Parameter. In Abbildung 6.12 wird der Einfluss von $k_{\text {ixi } \beta}$ auf die Ergebnisse der Trübungsdruckmodellierungen am Beispiel zweier Ethen-Polypropylacrylat-Copolymere mit unterschiedlichem Propylacrylatgehalt $\left(\mathrm{E}_{93.1} \mathrm{PA}_{6.9}\right.$ und $\left.\mathrm{E}_{73.8} \mathrm{PA}_{26.2}\right)$ illustriert.

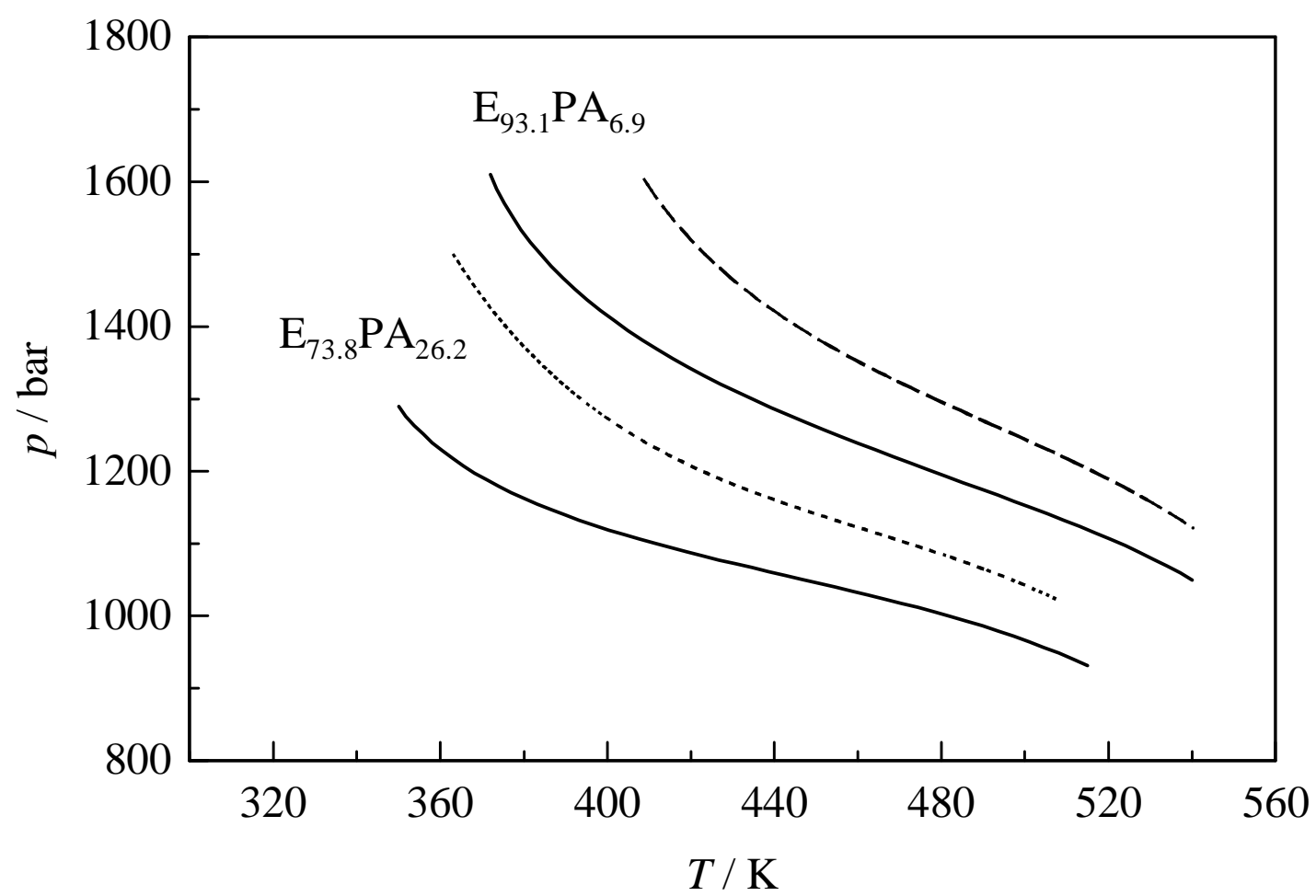

Abbildung 6.12: Berechnete Trübunsgdruckkurven für Mischungen aus Ethen und EthenPropylacrylat-Copolymeren mit unterschiedlichem Comonomergehalt. Die durchgezogenen Linien entsprechen den Modellierungsergebnissen bei Anpassung an experimentelle Daten $\left(k_{i \alpha} i \beta=0.010\right)$. Die gestrichelt bzw. punktiert gezeichneten Kurven stehen für die Ergebnisse von Modellierungen unter Anwendung von $k_{i \alpha i \beta}=0$.

Die gepunkteten bzw. gestrichelt dargestellten Linien stehen für Modellierungen für welche $k_{\mathrm{i} \alpha \mathrm{i} \beta}=0$ angenommen wird. Beide Kurven weichen signifikant von den durchgezogen gezeichneten Kurven ab, welche mit $k_{\mathrm{i} \alpha \mathrm{i} \beta}=0.010$ aus der Anpassung der experimentellen Trübungsdrücke berechnet wurden. Der Vergleich zeigt, dass die Größe des $k_{\text {i } \alpha i \beta}$-Wertes einen erheblichen Einfluss auf die Lage der berechneten Trübungsdruckwerte hat. 
Obwohl die im Rahmen dieser Arbeit erhaltenen $k_{\mathrm{i} \alpha \mathrm{i} \beta}$-Werte angepasste Größen sind, erscheint es interessant, die Abhängigkeit von $k_{\mathrm{i} \alpha \mathrm{i} \beta}$ von der Größe der Alkylestergruppe und vom Effekt der $\alpha$-Methylgruppe (verbunden mit dem Übergang von Acrylat- zu MethacrylatSystemen) $\mathrm{zu}$ untersuchen. In Abbildung 6.13 sind die ermittelten Werte für $k_{\mathrm{i} \alpha \mathrm{i} \beta}$ in Abhängigkeit von der Copolymerzusammensetzung dargestellt.

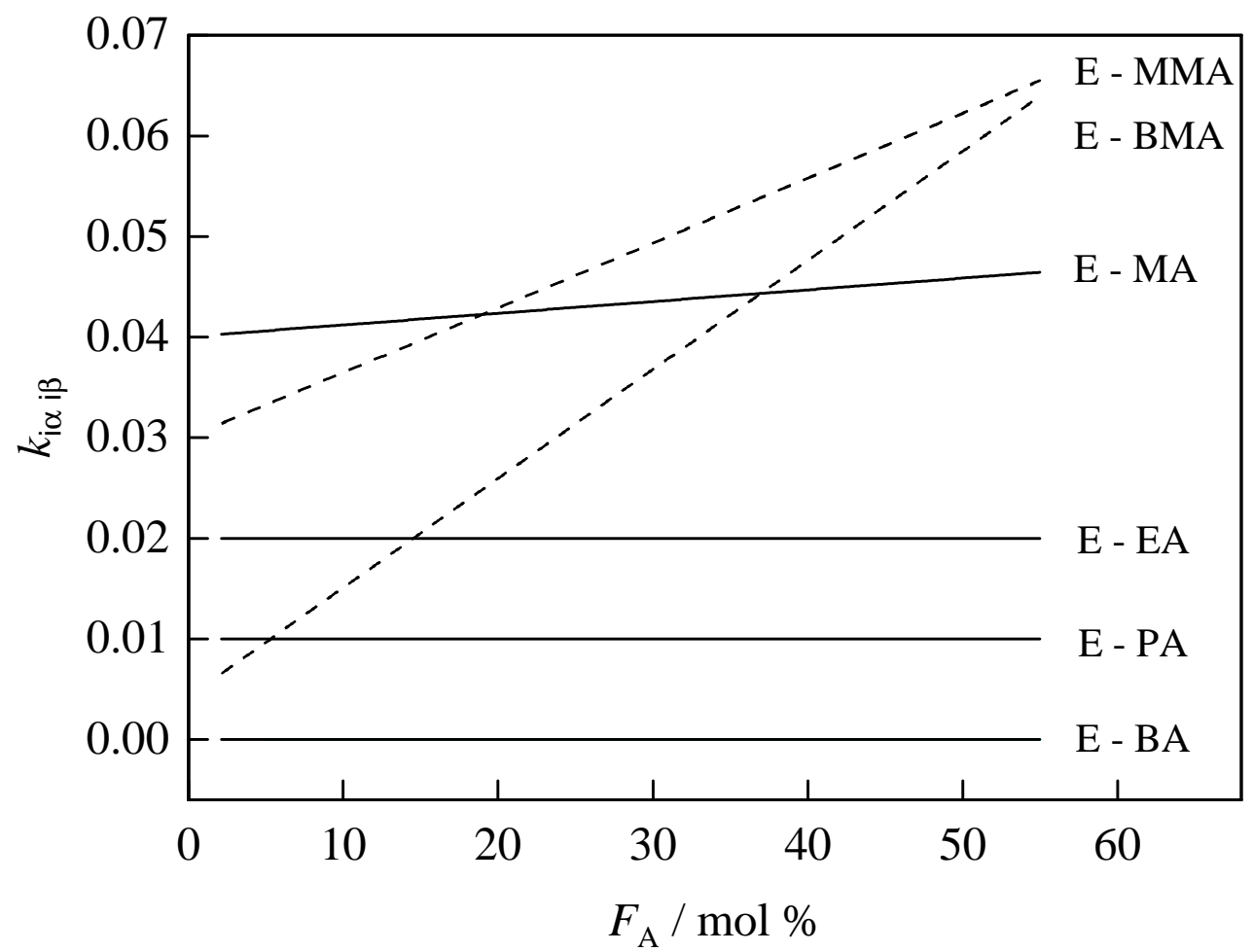

Abbildung 6.13: Abhängigkeit der binären Wechselwirkungsparameter $k_{i \alpha i \beta}$ von der Copolymerzusammensetzung für verschiedene Ethen-(Meth)Acrylat-Copolymersysteme. Ergebnisse erhalten aus PC-SAFT-Rechnungen angepasst an experimentelle Daten.

Während für E-MA eine lineare Abhängigkeit von $k_{\mathrm{i} \alpha \mathrm{i} \beta}$ von der Copolymerzusammensetzung beobachtet wird, sind die Werte für $k_{\mathrm{i} \alpha \mathrm{i} \beta}$ für E-BA, E-PA, und E-EA unabhängig von $F_{\mathrm{A}}$ und nehmen mit zunehmender Alkylestergröße ab. Für die $k_{\mathrm{i} \alpha \mathrm{i} \beta}$-Werte von E-MMA und E-BMA wird eine besonders ausgeprägte Abhängigkeit von $F_{\mathrm{A}}$ festgestellt. Interessanterweise sind die für verschwindenden Methacryaltgehalt extrapolierbaren $k_{\mathrm{i} \alpha \mathrm{i} \beta}$-Werte von E-MMA und EBMA den jeweiligen $k_{\text {i } \alpha \text { i }}$-Werten für die zugehörigen Ethen-Acrylat-Copolymeren mit derselben Alkylestergruppe nahezu gleich. 


\subsubsection{Abhängigkeit des Trübungsdrucks von der Molmasse}

Bei den in den vorangegangenen Abschnitten beschriebenen Modellierungen der Entmischungsdrücke für Ethen/Ethen-Copolymer-Systeme wurden die experimentell ermittelten Gewichtsmittel der Molmasse $M_{\mathrm{w}}$ der jeweils betrachteten Copolymerprobe berücksichtigt (vgl. 3.2.5). Nach der Bestimmung der Reinstoffparameter und der binären Wechselwirkungsparameter erschien es interessant, die Eignung des aufgestellten PC-SAFTModells zur Beschreibung der Molmassenabhängigkeit des Trübungsdrucks zu prüfen. Es wird an dieser Stelle darauf hingewiesen, dass die verschiedenen Reinstoff- und binären Parameter bei der Modellierung der Copolymer-Systeme hinsichtlich der möglichst guten Beschreibung der Abhängigkeit des Trübungsdrucks von der Copolymerzusammensetzung optimiert wurden. Es gehen daher keine systematischen Informationen zur Molmassenabhängigkeit in das Modell ein. Somit handelt es sich bei den im Folgenden beschriebenen Untersuchungen um einen Test der Voraussagefähigkeit des PC-SAFT-Modells.

Ergänzend zu den Messungen an Copolymersystemen wurden Trübungsdrücke für fünf Polyethylenproben mit deutlich unterschiedlichen Molmassen aufgenommen. Diese Proben wurden analog der für die Copolymere beschriebenen Vorgehensweise im Rahmen einer anderen Arbeit [19] ebenfalls bei 2000 bar in der in Abschnitt 3.1.4 beschriebenen MiniTechnikums-Anlage hergestellt. Die verschiedenen Molmassen der Proben wurden hierbei durch Variation der Synthesetemperatur und z.T. der Molmassenregler-Konzentration (Propionaldehyd) erhalten. Die Molmassenverteilungen wurden mittels Hochtemperatur-GPC (vgl. Abschnitt 3.2.5) bei der BASF AG (Ludwigshafen) bestimmt.

Die Ergebnisse der Messungen (Symbole) sind in Abbildung 6.14 gemeinsam mit modellierten Trübungskurven (durchgezogene Linien) dargestellt. Für die Modellierungen wurden die in Ref. [10] veröffentlichten Reinstoffparameter verwendet. Die Trübungsdrücke nehmen mit steigenden $M_{\mathrm{w}}$ zu. Die Charakteristik des Verlaufs der Trübungskurven ändert sich dabei nahezu nicht. Die Resultate der Modellrechnungen stimmen sehr gut mit den Messwerten überein. Lediglich für die Probe mit dem höchsten $M_{\mathrm{w}}$ wird keine vollständige Deckung der modellierten Kurve mit den experimentellen Daten gefunden. Unter Berücksichtigung der Unsicherheit der Molmassenbestimmung mittels der GPC-Methode (ca. $\pm 10 \%$ ) kann somit eine bemerkenswert gute Eignung des PC-SAFT-Modells zur Beschreibung der Molmassenabhängigkeit festgestellt werden. 


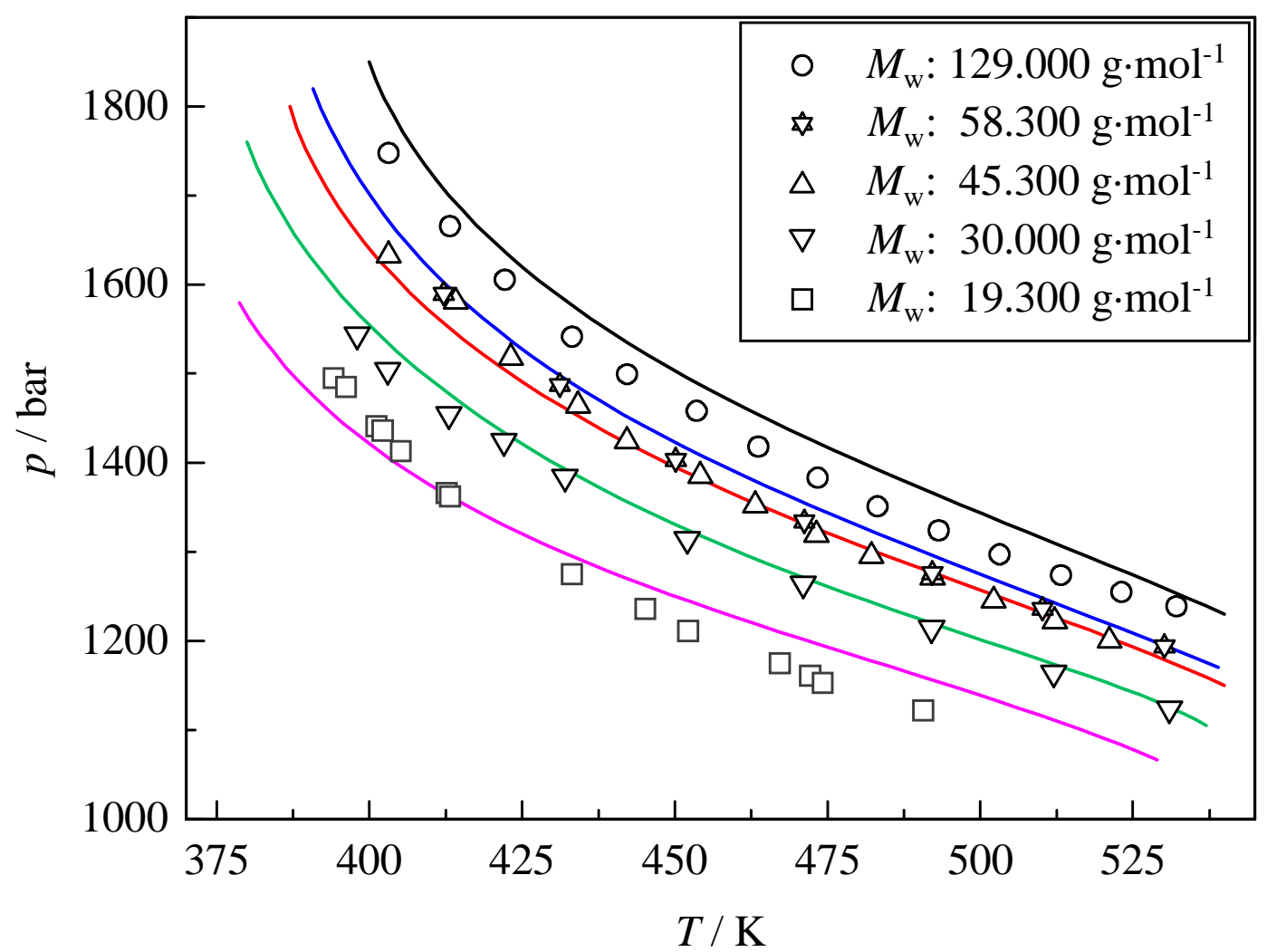

Abbildung 6.14: $\quad$ Experimentelle Trübungsdrücke (Symbole) von Polyethylen-Proben mit unterschiedlichem Gewichtsmittel der Molmasse, $M_{w}$ im Vergleich zu Modellrechnungen mit PC-SAFT (Linien).

Zum Test der Fähigkeit des PC-SAFT-Modells auch die Molmassenabhängigkeit für Copolymersysteme beschreiben $\mathrm{zu}$ können, wurden weitere Trübungsdruckmessungen ausgeführt. Für das System Ethen / E-EA wurden für fünf Proben mit einem EA-Gehalt von jeweils ca. 15 mol-\% Trübungsdruckexperimente durchgeführt. Es wird angemerkt, dass die unterschiedlichen Molmassen der E-EA-Proben zum Teil aus verschiedenen Synthesetemperaturen als auch durch Anwendung von Molmassenregler resultieren.

In Abbildung 6.15 ist die experimentell gefundene Abhängigkeit des Trübungsdrucks für Ethen-Ethylacrylat-Copolymere $\left(F_{\mathrm{A}}=15 \mathrm{~mol}-\%\right)$ vom Gewichtsmittel der Molmasse $M_{\mathrm{w}}$ für eine Temperatur von $200^{\circ} \mathrm{C}$ aufgetragen. Miteingezeichnet sind die schon in Abbildung 6.14 vorgestellten Ergebnisse für Polyethylen sowie zwei weitere Polyethylen-Literaturwerte $[13,20]$. Ausserdem werden Trübungsdrücke für Ethen-Methacrylsäure-Copolymere aus einer vorangegangenen Arbeit [21] gezeigt. Als durchgezogene Linien sind die jeweiligen, mit PC-SAFT für PE und E-EA berechneten Trübungsdrücke dargestellt. 
Aus der Auftragung wird deutlich, dass das PC-SAFT-Modell neben der guten Beschreibung des Ethen / Polyethylen-Systems in hervorragender Weise den Effekt der Molmasse auf den Trübungsdruck für Ethen-Ethylacrylat-Copolymere vorhersagen kann.

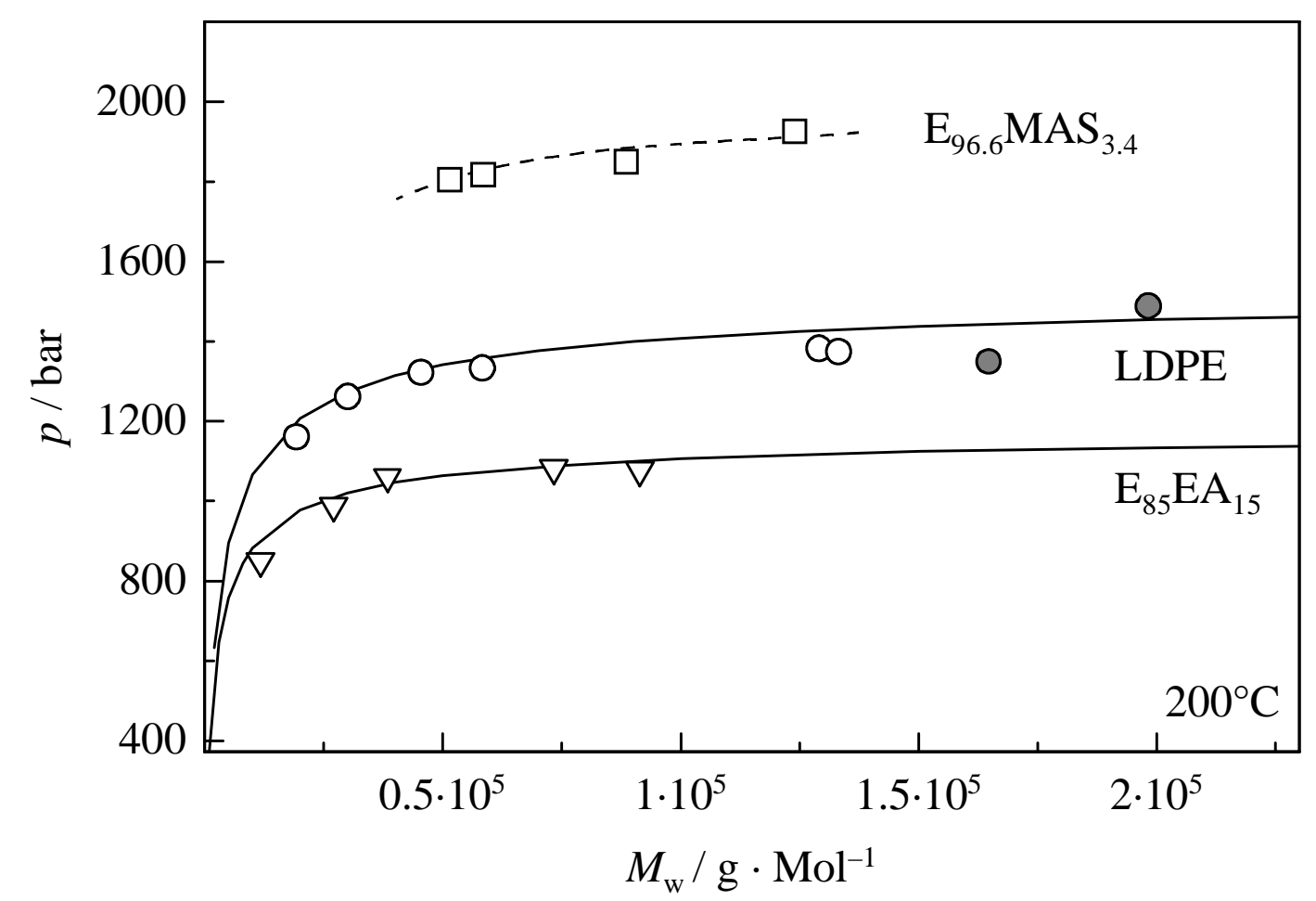

Abbildung 6.15: Abhängigkeit des Trübungsdrucks vom Gewichtsmittel, $M_{\mathrm{W}}$ der Molekulargewichtsverteilung. Dargestellt sind Ergebnisse für Polyethylen, EthenEthylacrylat-Copolymeren und Ethen-Methacrylsäure-Copolymeren. Grau gefüllte Symbol stehen für LDPE aus Referenz [13] und[20]. Die durchgezogenen Linien entsprechen Vorausberechnungen mit PC-SAFT.

Für die Trübungsdrücke von Polyethylen und E-EA wird unterhalb von $M_{\mathrm{w}}=40 \mathrm{~kg} / \mathrm{mol}$ eine starke, oberhalb eine verschwindende Abhängigkeit des Trübungsdrucks von der Molmasse festgestellt. Die mit abnehmender Molmasse zunehmende Löslichkeit ist vermutlich auf die ebenfalls abnehmende Dichte der Polymere zurückzuführen. So nimmt der Unterschied der Dichten von Polymer und überkritischen Solvens ab und ein für die Löslichkeit vorteilhaftes Verhältnis stellt sich bereits bei niedrigeren Drücken (weniger verdichtete Phase) ein. Die in Abbildung 6.15 für Polyethylen und E-EA-Copolymer gezeigten Verläufe des Trübungsdrucks sind abgesehen von der absoluten Lage nahezu identisch. Für E-MAS-Copolymere stehen Daten lediglich aus einem eingeschränkten $M_{\mathrm{w}}$-Bereich zur Verfügung. Illustriert durch die gestrichelt gezeichnete Hilfslinie deutet sich für E-MAS ein Verlauf an der dem der anderen Systemen sehr ähnlich ist. 


\subsection{Literatur Kapitel 6}

[1] J. Groß, Dissertation, Berlin (2001)

[2] G. Sadowski, Habilitationsschrift, Berlin (1999)

[3] W.G. Chapman, Ind. Eng. Chem. Res. 29 (1990) 1709

[4] J. M. Prausnitz, R. N. Lichtenthaler, E. G. Azevedo, Molecular Thermodynamics of Fluid Phase Equilibria, 2nd Edition, Prentice Hall. Englewood Cliffs, New Jersey (1986)

[5] W. G. Chapman, K. E. Gubbins, G. Jackson, M. Radosz, Fluid Phase Equilibria 52 (1989) 31

[6] J. A. Barker, D. Henderson, J. Chem. Phys. 47 (1967) 4714

[7] M. D. Amos, G. Jackson, Mol. Phys. 74 (1991) 191

[8] M. Banaszak, C. K. Chen, M. Radosz, Ind. Eng. Chem Res. 37 (1998) 4453

[9] K. P. Shukla, W. G. Chapman, Mol. Phys. 91 (1997) 1075

[10] Gross, J.; Sadowski, G., Ind. Eng. Chem. Res. 41 (2002) 1084

[11] Gross, J.; Spuhl, O.; Tumakaka, F.; Sadowski, G., Ind. Eng. Chem. Res. 42 (2003) 1266

[12] E. Narr, Diplomarbeit, Göttingen (1999)

[13] Byun, H. S.; Hasch, B. M.; McHugh, M. A .; Mähling, F.-O.; Busch, M; Buback, M., Macromolecules 29 (1996) 1625

[14] Chen, A.-Q.; Radosz, M., J. Chem. Eng. Data 44 (1999) 854

[15] Rodgers, P. A., J. Appl. Polym. Sci. 48 (1993) 1061

[16] T. Dröge, Dissertation Göttingen (1997)

[17] J. M. Prausnitz, Pure Appl. Chem. Vol. 72, 10 (2000) 1819

[18] Plessis, C.; Arzamendi, G.; Alberdi, J. M.; van Herk, A. M.; Leiza, J. R.; Asua, J. M., Macromol. Rapid Commun. 24 (2003) 173

[19] P. Becker, Dissertation Göttingen (2001)

[20] W. Müller, Dissertation Karlsruhe (1998)

[21] M. Buback, H. Latz, Macromol. Chem. Phys. 204 (2003) 638 


\section{Ausblick}

In dieser Arbeit wurde ein umfangreicher experimenteller Datensatz zu Trübungsdrücken binärer Mischungen aus Ethen und Ethen-(Meth)Acrylat-Copolymeren als auch aus Ethen bzw. Propen und Poly(meth)Acrylaten bereitgestellt. Anhand von Simulationsrechnungen mit der Perturbed-Chain-SAFT-Zustandsgleichung wurde die gute Eignung des theoretischen Modells zur Beschreibung der experimentellen Ergebnisse in weiten Bereichen von Druck, Temperatur und Copolymerzusammensetzung aufgezeigt. Darüber hinaus wurde an Beispielen die korrekte Wiedergabe des Einflusses der mittleren Polymermolmasse auf den Entmischungsdruck demonstriert. Hiermit ist eine gute Basis zur Beschreibung komplexerer Systeme geschaffen worden.

Insbesondere vor dem Hintergrund des technischen Interesses ist es sehr wichtig, die Modellrechnungen auf ternäre Systeme zu erweitern, da in der Praxis neben dem Monomer als auch Solvens Ethen und dem Copolymer in der Reaktionsmischung auch nicht umgesetztes Comonomer vorhanden ist. Erste Ergebnisse derartiger Studien sind vielversprechend [1]. Die in dieser Arbeit im direkten Anschluss an die Synthese und damit unter besonders praxisnahen Bedingungen erhaltenen experimentellen Daten für die Systeme E / EMA / E-EMA und E / BzMA / E-BzMA sind für die Validierung von Modellen für ternäre Mischungen besonders geeignet.

Bei der zukünftigen Weiterentwicklung des PC-SAFT-Modells sollten auch assoziierende Komponenten wie z.B. Säure- oder OH-Gruppen- und eventuell auch aromatische Funktionalität enthaltende Comonomere berücksichtigt werden. Dies würde die Berechnung z.B. des Entmischungsverhaltens von Systemen mit Ethen-(Meth)Acrylsäure-Copolymeren oder E-BzMA-Copolymeren erlauben. Um keine Fitparameter in das Modell einzuführen wäre es hierbei wünschenswert, unabhängig (z.B. spektroskopisch) bestimmte Werte für die assoziativen Wechselwirkungen zu verwenden. Gelingt die Beschreibung von Copolymeren mit (Meth)Acrylsäure, so bestünde eine weitere, besondere Herausforderung in der Modellierung der in dieser Arbeit vorgestellten Ergebnisse für ternäre Copolymere aus unpolaren, polaren und assoziierenden Monomeren (E-BMA-MAS).

Zur Verringerung des Rechenaufwands wurde für thermodynamische Modellierungen bisher überwiegend und so auch in dieser Arbeit die Verteilung des Molekulargewichts der Polymere vernachlässigt. Für die Rechnungen wird das Gewichtsmittel des Molekulargewichts verwendet und somit die polymere Komponente als quasi monodispers betracht. Da die Molmassenverteilung in vielen Fällen erheblich die Produkteigenschaften der 
Polymere bestimmt, sollte es bei der Fortentwicklung des PC-SAFT-Modells Ziel sein, die gesamte Information über die Molekulargewichtsverteilung in den Rechnungen $\mathrm{zu}$ berücksichtigen. Dies würde das Potential der PC-SAFT-Zustandsgleichung erheblich steigern, da somit auch der Abscheidungsprozess bei der Hochdruck-(Co)Polymerisation oder auch Polymerfraktionierungen simuliert werden könnten. Darüber hinaus wäre es interessant zu testen, inwieweit die Berücksichtigung der Polymermikrostruktur, gegeben durch Kurzund Langkettenverzweigungsgrade sowie die Sequenzlängenverteilung der Comonomere, die Vorhersagegenauigkeit erhöhen kann. Ein vermutlich erst langfristig erreichbares Ziel ist es, durch Integration der kinetischen und thermodynamischen Simulationswerkzeuge ein Modell $\mathrm{zu}$ erhalten mit dem der vollständige Produktionsprozess technischer Polymerisationen simuliert werden kann.

Die Untersuchungen zur Copolymerisationskinetik haben ergeben, dass innerhalb der Messgenauigkeit die verschiedenen Ethen-Methacrylat- als auch die Ethen-AcrylatCopolymer-Systeme bezüglich der Abhängigkeit der Copolymerzusammensetzung vom (Meth)Acrylatgehalt in der Monomermischung nicht unterschieden werden können. Es konnte allerdings für die Ethen-Acrylat-Systeme bei höheren Synthesetemperaturen aufgrund der Selbstinitierung der Comonomere nur ein beschränkter Zusammensetzungsbereich untersucht werden. Für ein detaillierteres Studium der Ethen-Acrylat-Familie sollte ein größerer $f_{\mathrm{A}^{-}} F_{\mathrm{A}^{-}}$ Datensatz bestimmt werden und Copolymere mit höherem Comonomergehalt synthetisiert werden. Um hierbei den Umsatz zu reduzieren, welcher bei hohen Temperaturen den maximalen erzielbaren Comonomergehalt im Copolymer beschränkt, könnte ein Inhibitor verwendet werden oder eine verringerte Reaktorverweilzeit gewählt werden.

Eine weitere reizvolle Fortführung der Studien zur Copolymerisationskinetik besteht darin die Mikrostruktur der synthetisierten Copolymere mittels quantitativer ${ }^{13} \mathrm{C}-\mathrm{NMR}$-Spektroskopie $\mathrm{zu}$ untersuchen. Hierbei sollte aufgrund der für die Systeme E-BA [2] und EMethylmethacrylat [3] beschriebenen Schwierigkeiten genau geprüft werden, ob die quantitative Auswertbarkeit der Spektren sichergestellt ist. Mit den aus den ${ }^{13} \mathrm{C}-\mathrm{NMR}$ Spektren bestimmbaren Kurz- und Langkettenverzweigungsgraden und den experimentellen Molmassenverteilung können analog [4] durch Modellierungen mit dem Programmpaket Predici $^{\circledR}$ die den Transfer-, „backbiting“- und „Scission“-Reaktionen zugeordneten Geschwindigkeitskoeffizienten berechnet werden. Hierdurch wird ein kinetisches Modell erhalten mit welchem die Produkteigenschaften der Copolymere in Abhängigkeit von den Synthesebedingungen simuliert werden können. 


\section{Literatur Ausblick}

[1] F. Tumakaka, G. Sadowski, M. Buback, E. Narr, H. Latz, Proceedings of AIChE 2003 Annual Meeting, San Francisco / USA, 11/2003

[2] M. Müller, Diplomarbeit, Göttingen (2000)

[3] L. Wittkowski, Dissertation, Göttingen (1998)

[4] H. C. M. van Boxtel, Dissertation, Göttingen (2000) 


\section{Anhang A}

\section{Verzeichnis der Abkürzungen}
A
Acrylat
A
präexponentieller Faktor, Frequenzfaktor
$B_{\mathrm{i}}$
integrale Molarabsorptivität der Substanz i
BA
n-Butylacrylat
BMA
n-Butylmethacrylat
BzMA
Benzylmethacrylat
CSTR
„Continous Stirred Tank Reaktor“ - Kontinuierlich betriebener Rührkessel
D $t$ BMP
2,6-di-tert-Butyl-4-Methylphenol
DtBP
Di-tert-Butyl-peroxid
E
Ethen
$E_{\mathrm{A}}$
Aktivierungsenergie
EA
Ethylacrylat
E-BMA
Poly(Ethen-co-butylmethacrylat)
E-BzMA
Poly(Ethen-co-benzylmethacrylat)
EMA
Ethylmethacrylat
E-MA
Poly(Ethen-co-Methylacrylat)
E-EA
Poly(Ethen-co-Ethylacrylat)
E-EMA
Poly(Ethen-co-Ethylmethacrylat)
E-EHA
Poly(Ethen-co-Ethylhexylacrylat)
E-PA
Poly(Ethen-co-Propylacrylat)
E-BA
Poly(Ethen-co-Butylacrylat)
E-MMA
Poly(Ethen-co-Methylmethacrylat)
EHA
2-Ethylhexylacrylat
EPUE
Explicit Penultimate $\underline{\text { Unit }} \underline{\text { Effect }}$
$f_{\mathrm{A}}$
Stoffmengenanteil der Komponente A in der Monomermischung
$F_{\mathrm{A}}$
Stoffmengenanteil der Komponente A im Copolymer
$J C I$
“Joint Confidence Interval” - gemeinsames Konfidenzintervall
Gew.-\%
Gewichtsprozent
GPC
Gel-Permeations-Chromatographie
IPUE
Implicit Penultimate $\underline{\text { Unit }} \underline{\mathrm{Effect}}$ 


\begin{tabular}{|c|c|}
\hline$k$ & Boltzmannkonstante \\
\hline$k_{\mathrm{ij}}$ & binärer Wechselwirkungsparameter \\
\hline$k_{\mathrm{pijk}}$ & $\begin{array}{l}\text { Wachstumsgeschwindigkeitskoeffizient für die Addition des } \\
\text { Monomeren k an ein Makroradikal mit } \mathrm{j} \text { als endständiger und } \mathrm{i} \text { als } \\
\text { vorletzter Monomereinheit }\end{array}$ \\
\hline$k_{\mathrm{t}}$ & Terminierungsgeschwindigkeitskoeffizient \\
\hline LCST & $\begin{array}{l}\text { „Lower Critical Solution Temperature“ - Unterer kritischer } \\
\text { Entmischungspunkt }\end{array}$ \\
\hline $\mathrm{L}$ & homogene flüssige Phase \\
\hline LDPE & Low Density Polyethylene, Polyethylen niedriger Dichte, s.a. PE \\
\hline LL & Flüssig-Flüssig-Phasengleichgewicht \\
\hline LM & Lösungsmittel \\
\hline$m$ & $\begin{array}{l}\text { Segmentzahl, Reinstoffparameter der Perturbed-Chain SAFT } \\
\text { Zustandsgleichung }\end{array}$ \\
\hline MA & Methylacrylat \\
\hline MAS & Methacrylsäure \\
\hline$M_{\mathrm{i}}$ & Molmasse der Komponente i \\
\hline$\dot{m}$ & Massenfluss der Komponente i \\
\hline MMA & Methylmethacrylat \\
\hline$M_{\mathrm{n}}$ & Zahlenmittelwert der Molmasse \\
\hline$M_{\mathrm{p}}$ & mittlere Molmasse einer Monomereinheit im Copolymer \\
\hline$M_{\mathrm{w}}$ & Gewichtsmittelwert der Molmasse \\
\hline NMR & $\underline{\text { Nuclear-Magnetic-Resonance-Spektroskopie }}$ \\
\hline$n_{\mathrm{i}}$ & Stoffmenge von Komponente i \\
\hline$\dot{n}_{\mathrm{A}}$ & (Meth)Acrylat-Stoffmengenfluss \\
\hline$\dot{n}_{\mathrm{E}}$ & Ethen-Stoffmengenfluss \\
\hline$\dot{n}_{\mathrm{P}}$ & Copolymer-Stoffmengenfluss \\
\hline$p$ & Druck \\
\hline PA & Propylacrylat \\
\hline PC-SAFT & Perturbed $\underline{\text { Chain }} \underline{\text { Statistical }} \underline{\text { Associating }}$ Fluid Theory \\
\hline $\mathrm{PE}$ & Polyethylen \\
\hline Poly(MA) & Polymethylacrylat \\
\hline Poly(EA) & Polyethylacrylat \\
\hline Poly(EMA) & Polyethylmethacrylat \\
\hline Poly(PA) & Polypropylacrylat \\
\hline Poly(BA) & Polybutylacrylat \\
\hline
\end{tabular}




\begin{tabular}{|c|c|}
\hline Poly(MMA) & Polymethylmethacrylat \\
\hline Poly(BMA) & Polybutylmethacrylat \\
\hline Poly(BzMA) & Polybenzylmethacrylat \\
\hline$r_{\mathrm{i}}$ & Copolymerisationsparameter \\
\hline$s$ & Radikal-Copolymerisationsparameter \\
\hline SAFT & 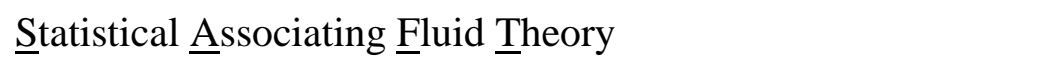 \\
\hline ss & 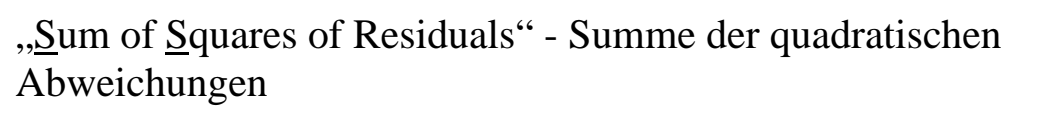 \\
\hline SSS & 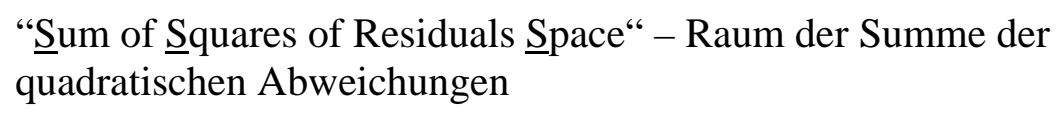 \\
\hline$R$ & Allgemeine Gaskonstante \\
\hline$T$ & Absolute Temperatur \\
\hline UCST & 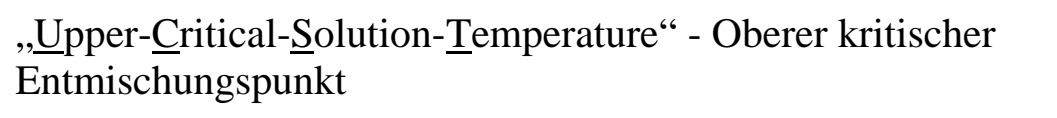 \\
\hline$w_{\mathrm{i}}$ & Gewichtungsfaktor für den Datenpunkt i \\
\hline
\end{tabular}

\section{Griechische Symbole}

$\varepsilon$

$\delta$

$\sigma$

$\theta$
Potentialtiefe der dispersiven Anziehung, der Quotient ( $\varepsilon / \mathrm{k})$ ist ein Reinstoffparameter der Perturbed-Chain SAFT Zustandsgleichung chemische Verschiebung temperaturunabhängiger Segmentdurchmesser, Reinstoffparameter der Perturbed-Chain-SAFT-Zustandsgleichung

Temperatur in ${ }^{\circ} \mathrm{C}$ 


\section{Anhang B}

Aufstellungen der für Trübungsdruckmessungen verwendeten Polymere. Angegeben werden: Zusammensetzung, Synthesebedingungen (Druck und Temperatur), Zahlen- und Gewichtsmittel der Molmassen $\left(M_{\mathrm{n}}\right.$ bzw. $\left.M_{\mathrm{w}}\right)$ und die Polydispersität.

\begin{tabular}{|c|c|c|c|c|c|c|c|}
\hline \multirow[t]{2}{*}{ Bezeichnung } & \multicolumn{2}{|c|}{ Copolymerzusammensetzung } & \multirow{2}{*}{$\begin{array}{c}\text { Synthese- } \\
\text { temperatur } \\
\text { K }\end{array}$} & \multirow{2}{*}{$\begin{array}{c}\text { Synthese- } \\
\text { Druck } \\
\text { bar }\end{array}$} & \multicolumn{2}{|c|}{ Molmasse } & \multirow{2}{*}{$\begin{array}{c}\begin{array}{c}\text { Poly- } \\
\text { dispersität }\end{array} \\
Q\end{array}$} \\
\hline & Comonomer & $\mathrm{mol} \%$ & & & $\begin{array}{c}M_{\mathrm{n}} \\
\mathrm{g} \cdot \mathrm{mol}^{-1}\end{array}$ & $\begin{array}{c}M_{\mathrm{w}} \\
\mathrm{g} \cdot \mathrm{mol}^{-1}\end{array}$ & \\
\hline PE 1 & - & - & 429 & 2000 & 33000 & 129000 & 3.9 \\
\hline PE 2 & - & - & 469 & 2000 & 19900 & 58300 & 2.9 \\
\hline PE 3 & - & - & 509 & 2000 & 12000 & 45300 & 3.8 \\
\hline PE 4 & - & - & 477 & 2000 & 9500 & 30000 & 3.2 \\
\hline PE 5 & - & - & 430 & 2000 & 6300 & 19300 & 3.1 \\
\hline $\mathrm{E}_{96.4} \mathrm{EA}_{3.6}$ & Ethylacrylat & 3.6 & 473 & 2000 & n.best. & n.best. & n.best. \\
\hline $\mathrm{E}_{93.7} \mathrm{EA}_{6.3}$ & Ethylacrylat & 6.3 & 473 & 2000 & n.best. & n.best. & n.best. \\
\hline $\mathrm{E}_{76.6} \mathrm{EA}_{23.4}$ & Ethylacrylat & 23.4 & 443 & 2000 & 78400 & 156600 & 2.0 \\
\hline $\mathrm{E}_{71.6} \mathrm{EA}_{28.4}$ & Ethylacrylat & 28.4 & 473 & 2000 & 64400 & 116700 & 1.8 \\
\hline $\mathrm{E}_{65.2 .} \mathrm{EA}_{34.8}$ & Ethylacrylat & 34.8 & 443 & 2000 & 75900 & 159500 & 2.1 \\
\hline $\mathrm{E}_{54.3} \mathrm{EA}_{45.7}$ & Ethylacrylat & 45.7 & 443 & 2000 & 67600 & 142900 & 2.1 \\
\hline $\mathrm{E}_{93.1} \mathrm{PA}_{6.9}$ & Propylacrylat & 6.9 & 453 & 2000 & n.best. & n.best. & n. best. \\
\hline $\mathrm{E}_{85.9} \mathrm{PA}_{14.1}$ & Propylacrylat & 14.1 & 453 & 2000 & 83000 & 147300 & 1.8 \\
\hline $\mathrm{E}_{81.1} \mathrm{PA}_{18.9}$ & Propylacrylat & 18.9 & 473 & 2000 & 54200 & 112400 & 2.1 \\
\hline $\mathrm{E}_{73.8} \mathrm{PA}_{26.2}$ & Propylacrylat & 26.2 & 453 & 2000 & 58100 & 127000 & 2.2 \\
\hline $\mathrm{E}_{68.8} \mathrm{PA}_{31.2}$ & Propylacrylat & 31.2 & 453 & 2000 & 78400 & 128600 & 1.6 \\
\hline $\mathrm{E}_{62.6} \mathrm{PA}_{37.4}$ & Propylacrylat & 37.4 & 453 & 2000 & 49000 & 126200 & 2.6 \\
\hline $\mathrm{E}_{73.2} \mathrm{BA}_{26.8}$ & Butylacrylat & 26.8 & 513 & 2000 & 72500 & 159500 & 2.2 \\
\hline $\mathrm{E}_{90.4} \mathrm{MMA}_{9.6}$ & Methylmethacrylat & 9.6 & 443 & 2000 & n.best. & n.best. & n. best. \\
\hline $\mathrm{E}_{83.1} \mathrm{MMA}_{16.9}$ & Methylmethacrylat & 16.9 & 443 & 2000 & 41400 & 67100 & 1.6 \\
\hline $\mathrm{E}_{81.5} \mathrm{MMA}_{18.5}$ & Methylmethacrylat & 18.5 & 513 & 2000 & 10900 & 20000 & 1.8 \\
\hline $\mathrm{E}_{64.9} \mathrm{MMA}_{35.1}$ & Methylmethacrylat & 35.1 & 443 & 2000 & 49800 & 83500 & 1.7 \\
\hline $\mathrm{E}_{58.5} \mathrm{MMA}_{41.5}$ & Methylmethacrylat & 41.5 & 443 & 2000 & 28610 & 52600 & 1.8 \\
\hline $\mathrm{E}_{50.4} \mathrm{MMA}_{49.6}$ & Methylmethacrylat & 49.6 & 473 & 2000 & 31900 & 62300 & 2.0 \\
\hline $\mathrm{E}_{45.0} \mathrm{MMA}_{55.0}$ & Methylmethacrylat & 55.0 & 443 & 2000 & 14800 & 40700 & 2.8 \\
\hline
\end{tabular}




\begin{tabular}{|c|c|c|c|c|c|c|c|}
\hline \multirow[t]{2}{*}{ Bezeichnung } & \multicolumn{2}{|c|}{ Copolymerzusammensetzung } & \multirow{2}{*}{$\begin{array}{c}\text { Synthese- } \\
\text { temperatur } \\
\text { K }\end{array}$} & \multirow{2}{*}{$\begin{array}{c}\text { Synthese- } \\
\text { Druck } \\
\text { bar }\end{array}$} & \multicolumn{2}{|c|}{ Molmasse } & \multirow{2}{*}{$\begin{array}{c}\begin{array}{c}\text { Poly- } \\
\text { dispersität } \\
Q\end{array} \\
\end{array}$} \\
\hline & Comonomer & $\operatorname{mol} \%$ & & & $\begin{array}{c}M_{\mathrm{n}} \\
\mathrm{g} \cdot \mathrm{mol}^{-1}\end{array}$ & $\begin{array}{c}M_{\mathrm{w}} \\
\mathrm{g} \cdot \mathrm{mol}^{-1}\end{array}$ & \\
\hline $\mathrm{E}_{89} \mathrm{EMA}_{11}$ & Ethylmethacrylat & 11 & 473 & 2000 & 25500 & 47300 & 1.9 \\
\hline $\mathrm{E}_{81} \mathrm{EMA}_{19}$ & Ethylmethacrylat & 19 & 473 & 2000 & 24800 & 45400 & 1.8 \\
\hline $\mathrm{E}_{74} \mathrm{EMA}_{26}$ & Ethylmethacrylat & 26 & 473 & 2000 & 22500 & 42000 & 1.9 \\
\hline $\mathrm{E}_{71} \mathrm{EMA}_{29}$ & Ethylmethacrylat & 29 & 473 & 2000 & 25100 & 48700 & 1.9 \\
\hline $\mathrm{E}_{62} \mathrm{EMA}_{38}$ & Ethylmethacrylat & 38 & 473 & 2000 & 24000 & 44300 & 1.8 \\
\hline $\mathrm{E}_{93.3} \mathrm{BMA}_{6.7}$ & Butylmethacrylat & 6.7 & 473 & 2000 & n. best. & n. best. & n. best. \\
\hline $\mathrm{E}_{88.3} \mathrm{BMA}_{11.7}$ & Butylmethacrylat & 11.7 & 473 & 2000 & 23400 & 39800 & 1.7 \\
\hline $\mathrm{E}_{81.5} \mathrm{BMA}_{18.5}$ & Butylmethacrylat & 18.5 & 473 & 2000 & 24800 & 41000 & 1.7 \\
\hline $\mathrm{E}_{87.3} \mathrm{BMA}_{22.7}$ & Butylmethacrylat & 22.7 & 473 & 2000 & 24500 & 44400 & 1.8 \\
\hline $\mathrm{E}_{66.2} \mathrm{BMA}_{33.8}$ & Butylmethacrylat & 33.8 & 473 & 2000 & 55000 & 96700 & 1.7 \\
\hline $\mathrm{E}_{56.0} \mathrm{BMA}_{44.0}$ & Butylmethacrylat & 44.0 & 473 & 2000 & 58900 & 101700 & 1.7 \\
\hline $\mathrm{E}_{96.7} \mathrm{BzMA}_{3.3}$ & Benzylmethacrylat & 3.3 & 473 & 2000 & n.best. & n.best. & n.best. \\
\hline $\mathrm{E}_{87.3} \mathrm{BzMA}_{12.7}$ & Benzylmethacrylat & 12.7 & 473 & 2000 & 47100 & 73500 & 1.6 \\
\hline $\mathrm{E}_{80.0} \mathrm{BzMA}_{20.0}$ & Benzylmethacrylat & 20.0 & 473 & 2000 & 36900 & 60700 & 1.6 \\
\hline $\mathrm{E}_{74.9} \mathrm{BzMA}_{26.1}$ & Benzylmethacrylat & 26.1 & 473 & 2000 & 36900 & 61100 & 1.7 \\
\hline $\mathrm{E}_{86.9} \mathrm{BzMA}_{13.1}$ & Benzylmethacrylat & 13.1 & 473 & 2000 & n.best. & n.best. & n.best. \\
\hline $\mathrm{E}_{79.7} \mathrm{BzMA}_{20.3}$ & Benzylmethacrylat & 20.3 & 473 & 2000 & n.best. & n.best. & n.best. \\
\hline $\mathrm{E}_{74.8} \mathrm{BzMA}_{25.2}$ & Benzylmethacrylat & 25.2 & 473 & 2000 & n.best. & n.best. & n.best. \\
\hline $\mathrm{E}_{69.7} \mathrm{BzMA}_{30.4}$ & Benzylmethacrylat & 30.4 & 473 & 2000 & 35600 & 62200 & 1.7 \\
\hline $\mathrm{E}_{66.6} \mathrm{BzMA}_{33.5}$ & Benzylmethacrylat & 33.5 & 473 & 2000 & n.best. & n.best. & n.best. \\
\hline Poly(MA) & Methylacrylat & 100 & 343 & 1 & 76500 & 186900 & 2.4 \\
\hline Poly(EA) ${ }^{1}$ & Ethylacrylat & 100 & n. bekannt & 1 & 85400 & 153700 & 1.8 \\
\hline $\operatorname{Poly}(\mathrm{PA})^{1}$ & Propylacrylat & 100 & n. bekannt & 1 & 47900 & 108300 & 2.3 \\
\hline Poly(MMA) & Methylmethacrylat & 100 & 343 & 1 & 71200 & 104700 & 1.5 \\
\hline Poly(BMA) & Butylmethacrylat & 100 & 343 & 1 & 38100 & 65500 & 1.7 \\
\hline
\end{tabular}

\footnotetext{
${ }^{1}$ bezogen von Scientific Polymer Products Inc., Ontario / USA
} 


\begin{tabular}{|c|c|c|c|c|}
\hline \multirow[t]{2}{*}{ Bezeichnung } & \multicolumn{2}{|c|}{ Copolymerzusammensetzung } & \multirow{2}{*}{$\begin{array}{c}\text { Synthese- } \\
\text { temperatur }^{2} \\
\mathrm{~K}\end{array}$} & \multirow{2}{*}{$\begin{array}{c}\text { Synthese- } \\
\text { Druck } \\
\text { bar }\end{array}$} \\
\hline & mol \% BMA & mol $\%$ MAS & & \\
\hline $\mathrm{E}_{93.0} \mathrm{MAS}_{7.0}$ & 0 & 7.0 & n. bestimmt ${ }^{3}$ & n. bestimmt ${ }^{3}$ \\
\hline $\mathrm{E}_{93.0} \mathrm{BMA}_{0.4} \mathrm{MAS}_{6.6}$ & 0.4 & 6.6 & n. bestimmt ${ }^{3}$ & n. bestimmt ${ }^{3}$ \\
\hline $\mathrm{E}_{93.0} \mathrm{BMA}_{0.6} \mathrm{MAS}_{6.4}$ & 0.6 & 6.4 & n. bestimmt ${ }^{3}$ & n. bestimmt ${ }^{3}$ \\
\hline $\mathrm{E}_{93.0} \mathrm{BMA}_{0.9} \mathrm{MAS}_{6.1}$ & 0.9 & 6.1 & n. bestimmt ${ }^{3}$ & n. bestimmt ${ }^{3}$ \\
\hline $\mathrm{E}_{92.8} \mathrm{MAS}_{7.2}$ & 0 & 7.2 & 533 & 2000 \\
\hline $\mathrm{E}_{99.0} \mathrm{MAS}_{1.0}$ & 0 & 1.0 & 533 & 2000 \\
\hline $\mathrm{E}_{92.9} \mathrm{BMA}_{0.8} \mathrm{MAS}_{6.3}$ & 0.8 & 6.3 & 533 & 2000 \\
\hline $\mathrm{E}_{92.7} \mathrm{BMA}_{1.7} \mathrm{MAS}_{5.6}$ & 1.7 & 5.6 & 533 & 2000 \\
\hline $\mathrm{E}_{92.7} \mathrm{BMA}_{3.5} \mathrm{MAS}_{3.8}$ & 3.5 & 3.8 & 533 & 2000 \\
\hline $\mathrm{E}_{92.9} \mathrm{BMA}_{4.2} \mathrm{MAS}_{2.9}$ & 4.2 & 2.9 & 533 & 2000 \\
\hline $\mathrm{E}_{92.7} \mathrm{BMA}_{5.5} \mathrm{MAS}_{1.8}$ & 5.5 & 1.8 & 533 & 2000 \\
\hline $\mathrm{E}_{92.9} \mathrm{BMA}_{6.2} \mathrm{MAS}_{0.9}$ & 6.2 & 0.9 & 533 & 2000 \\
\hline $\mathrm{E}_{92.9} \mathrm{BMA}_{6.8} \mathrm{MAS}_{0.3}$ & 14.1 & 0.3 & 533 & 2000 \\
\hline $\mathrm{E}_{87.2} \mathrm{BMA}_{5.5} \mathrm{MAS}_{7.3}$ & 5.5 & 7.3 & 533 & 2000 \\
\hline $\mathrm{E}_{79.6} \mathrm{BMA}_{13.3} \mathrm{MAS}_{7.1}$ & 13.3 & 7.1 & 533 & 2000 \\
\hline $\mathrm{E}_{93.5} \mathrm{BMA}_{6.2} \mathrm{MAS}_{0.3}$ & 6.2 & 0.3 & 533 & 2000 \\
\hline $\mathrm{E}_{92.2} \mathrm{BMA}_{7.8}$ & 0 & 7.8 & 533 & 2000 \\
\hline
\end{tabular}

\footnotetext{
${ }^{2}$ Die Synthesetemperatur entspricht der Temperatur während der Hochdruck-Copolymerisation

${ }^{3}$ Das Ausgangsmaterial ist ein kommerzielles Produkt (Fa. DuPont)
}

\section{Syntheseanweisung Poly(Meth)Acrylate:}

Es werden $10 \mathrm{~g}$ durch Destillation aufgereinigtes Monomer in $30 \mathrm{ml}$ Toluol gelöst. Die Mischung wurde unter Rühren auf $70^{\circ} \mathrm{C}$ erhitzt und mit $0.12 \mathrm{~g}$ AIBN versetzt. Anschließend wurde in Zeitabständen von ca. 15 min. Proben genommen. Eine eindeutige Eintrübung bei Zugabe von Methanol zeigt ausreichenden Umsatz an. Das unter weiterer Zugabe von Methanol ausgefällte Produkt wird zunächst unter vermindertem Druck von restlichem Monomer, Toluol und Methanol befreit. Abschließend wurde für 5 Tage im Feinvakuum $\left(<10^{-3}\right.$ mbar) getrocknet. 


\section{Anhang C}

Experimentelle Trübungsdrücke für Ethen / Polymer-Mischungen, Polymerkonzentration $5.0 \pm 0.3$ Gew.\%

\begin{tabular}{|c|c|c|c|c|c|c|c|}
\hline \multicolumn{2}{|c|}{ Ethen / PE 1} & \multicolumn{2}{|c|}{ Ethen / PE 2} & \multicolumn{2}{|c|}{ Ethen / PE 3} & \multicolumn{2}{|c|}{ Ethen / PE 4} \\
\hline$T / \mathrm{K}$ & $p /$ bar & $T / \mathrm{K}$ & $p /$ bar & $T / \mathrm{K}$ & $p /$ bar & $T / \mathrm{K}$ & $p /$ bar \\
\hline 394.15 & 1495 & 398 & 1540 & 403.15 & 1637 & 403.15 & 1748 \\
\hline 396.15 & 1485 & 403 & 1500 & 414.15 & 1585 & 413.15 & 1666 \\
\hline 401.15 & 1441 & 413 & 1450 & 423.15 & 1522 & 422.15 & 1606 \\
\hline 402.15 & 1436 & 422 & 1420 & 434.15 & 1468 & 433.15 & 1542 \\
\hline 405.15 & 1413 & 432 & 1380 & 442.15 & 1428 & 442.15 & 1499.5 \\
\hline 412.65 & 1366 & 452 & 1310 & 454.15 & 1389 & 453.55 & 1458 \\
\hline 413.15 & 1362 & 471 & 1260 & 463.15 & 1356 & 463.65 & 1418 \\
\hline 433.15 & 1275 & 492 & 1210 & 473.15 & 1323 & 473.35 & 1383 \\
\hline 445.15 & 1236 & & & 482.15 & 1299 & 483.15 & 1351 \\
\hline 452.15 & 1211 & & & 492.15 & 1275 & 493.15 & 1324 \\
\hline 467.15 & 1175 & & & 502.15 & 1249 & 503.15 & 1297 \\
\hline 472.15 & 1161 & & & 512.15 & 1226 & 513.15 & 1274 \\
\hline 474.15 & 1153 & & & 521.15 & 1204 & 523.15 & 1255 \\
\hline 490.65 & 1122 & & & & & 532.15 & 1239 \\
\hline
\end{tabular}

\begin{tabular}{|c|c|c|c|c|c|c|c|}
\hline \multicolumn{2}{|c|}{ Ethen / PE 5} & \multicolumn{2}{|c|}{ Ethen / $\mathrm{E}_{96.4} \mathrm{EA}_{3.6}$} & \multicolumn{2}{|c|}{ Ethen / $\mathrm{E}_{93.7} \mathrm{EA}_{6.3}$} & \multicolumn{2}{|c|}{ Ethen / $\mathrm{E}_{76.6} \mathrm{EA}_{23.4}$} \\
\hline$T / \mathrm{K}$ & $p /$ bar & $T / \mathrm{K}$ & $p /$ bar & $T / \mathrm{K}$ & $p /$ bar & $T / \mathrm{K}$ & $p /$ bar \\
\hline 412.15 & 1590 & 384.05 & 1631 & 372.15 & 1510 & 353.15 & 1280 \\
\hline 431.15 & 1487 & 395.15 & 1568 & 393.15 & 1420 & 364.15 & 1256 \\
\hline 450.15 & 1403 & 414.85 & 1471 & 413.15 & 1351 & 373.75 & 1236 \\
\hline 471.15 & 1334 & 435.15 & 1394 & 433.15 & 1295 & 393.15 & 1190 \\
\hline 492.15 & 1276 & 454.15 & 1336 & 452.15 & 1252 & 413.15 & 1155 \\
\hline 510.15 & 1236 & 473.15 & 1288 & 472.55 & 1212 & 434.15 & 1123 \\
\hline \multirow[t]{5}{*}{530.15} & 1194 & 492.15 & 1247 & 492.15 & 1181 & 454.15 & 1095 \\
\hline & & 514.15 & 1207 & 511.15 & 1147 & 473.15 & 1072 \\
\hline & & 531.15 & 1175 & 531.35 & 1122 & 493.65 & 1047 \\
\hline & & & & & & 513.55 & 1025 \\
\hline & & & & & & 533.15 & 1000 \\
\hline
\end{tabular}




\begin{tabular}{|cc|cc|cc|cc|}
\hline \multicolumn{2}{|c|}{ Ethen / $\mathrm{E}_{71.6} \mathrm{EA}_{28.4}$} & \multicolumn{2}{|c|}{ Ethen / $\mathrm{E}_{65.2} \mathrm{EA}_{34.8}$} & \multicolumn{2}{|c|}{ Ethen / $\mathrm{E}_{54.3} \mathrm{EA}_{45.7}$} & \multicolumn{2}{c|}{ Ethen / Poly(EA) } \\
\hline$T / \mathrm{K}$ & $p / \mathrm{bar}$ & $T / \mathrm{K}$ & $p / \mathrm{bar}$ & $T / \mathrm{K}$ & $p / \mathrm{bar}$ & $T / \mathrm{K}$ & $p / \mathrm{bar}$ \\
\hline 363.15 & 1236 & 366.65 & 1296 & 372.75 & 1324 & 354.15 & 1613 \\
373.15 & 1206 & 374.65 & 1272 & 391.95 & 1265 & 364.15 & 1541 \\
383.15 & 1183 & 397.15 & 1219 & 412.15 & 1217 & 373.15 & 1485 \\
393.15 & 1165 & 412.15 & 1191 & 433.15 & 1173 & 393.15 & 1376 \\
413.15 & 1131 & 434.15 & 1151 & 453.15 & 1135 & 414.35 & 1288 \\
433.15 & 1100 & 454.65 & 1120 & 472.15 & 1104 & 434.65 & 1217 \\
453.15 & 1072 & 474.65 & 1090 & 491.15 & 1076 & 453.85 & 1162 \\
473.15 & 1048 & 491.15 & 1066 & 511.15 & 1048 & 473.45 & 1117 \\
493.15 & 1022 & 513.15 & 1039 & 529.15 & 1025 & 492.35 & 1077 \\
513.15 & 100 & 532.15 & 1018 & & & 513.55 & 1038 \\
& & & & & 533.15 & 1010 \\
\hline
\end{tabular}

\begin{tabular}{|cc|cc|cc|cc|}
\hline \multicolumn{2}{|c|}{ Ethen / $\mathrm{E}_{93.1} \mathrm{PA}_{6.9}$} & \multicolumn{2}{|c|}{ Ethen / $\mathrm{E}_{85.9} \mathrm{PA}_{14.1}$} & \multicolumn{2}{|c|}{ Ethen / $\mathrm{E}_{81.1} \mathrm{PA}_{18.9}$} & \multicolumn{2}{|c|}{ Ethen / $\mathrm{E}_{73.8} \mathrm{PA}_{26.2}$} \\
\hline$T / \mathrm{K}$ & $p / \mathrm{bar}$ & $T / \mathrm{K}$ & $p / \mathrm{bar}$ & $T / \mathrm{K}$ & $p / \mathrm{bar}$ & $T / \mathrm{K}$ & $p / \mathrm{bar}$ \\
\hline 366.45 & 1527 & 353.15 & 1380 & 367.15 & 1250 & 354.15 & 1208 \\
376.15 & 1476 & 363.15 & 1330 & 373.15 & 1226 & 374.15 & 1182 \\
386.65 & 1433 & 373.65 & 1297 & 393.15 & 1183 & 392.15 & 1147 \\
393.15 & 1408 & 397.85 & 1234 & 412.15 & 1153 & 413.15 & 1112 \\
413.15 & 1338 & 415.45 & 1192 & 431.15 & 1124 & 433.15 & 1083 \\
423.15 & 1310 & 434.15 & 1150 & 451.15 & 1097 & 451.15 & 1062 \\
442.15 & 1262 & 452.15 & 1127 & 468.15 & 1077 & 471.15 & 1038 \\
452.15 & 1244 & 473.15 & 1098 & 491.55 & 1055 & 491.15 & 1017 \\
472.15 & 1204 & 492.15 & 1074 & 512.15 & 1031 & 512.15 & 995 \\
491.15 & 1173 & 509.15 & 1056 & 529.85 & 1018 & 530.15 & 978 \\
511.15 & 1141 & 532.15 & 1035 & & & & \\
531.15 & 1115 & & & & & \\
\hline
\end{tabular}




\begin{tabular}{|cc|cc|cc|cc|}
\hline \multicolumn{2}{|c|}{ Ethen / $\mathrm{E}_{68.8} \mathrm{PA}_{31.2}$} & \multicolumn{2}{|c|}{ Ethen / $\mathrm{E}_{26.6} \mathrm{PA}_{37.4}$} & \multicolumn{2}{|c|}{ Ethen / Poly $\mathrm{PA})$} & \multicolumn{2}{|c|}{ Ethen / $\mathrm{E}_{90.4} \mathrm{MMA}_{9.6}$} \\
\hline$T / \mathrm{K}$ & $p / \mathrm{bar}$ & $T / \mathrm{K}$ & $p / \mathrm{bar}$ & $T / \mathrm{K}$ & $p / \mathrm{bar}$ & $T / \mathrm{K}$ & $p / \mathrm{bar}$ \\
\hline 351.55 & 1215 & 351.45 & 1218 & 352.95 & 1093 & 372.45 & 1510 \\
362.45 & 1186 & 365.15 & 1193 & 363.25 & 1059 & 393.25 & 1312 \\
373.15 & 1163 & 375.15 & 1172 & 373.25 & 1033 & 402.55 & 1290 \\
393.15 & 1129 & 391.15 & 1139 & 393.15 & 992 & 412.05 & 1255 \\
412.35 & 1100 & 412.15 & 1106 & 412.15 & 961 & 422.45 & 1227 \\
432.65 & 1069 & 434.15 & 1072 & 432.15 & 936 & 432.05 & 1185 \\
452.15 & 1046 & 453.15 & 1050 & 452.15 & 914 & 451.65 & 1145 \\
472.65 & 1024 & 471.65 & 1029 & 472.15 & 892 & 472.35 & 1109 \\
492.15 & 1003 & 492.15 & 1008 & 491.15 & 881 & 491.85 \\
510.15 & 985 & 512.15 & 987 & 511.15 & 877 & 511.55 & 1076 \\
530.15 & 964 & 528.15 & 974 & 531.15 & 874 & 527.05 & 1063 \\
\hline
\end{tabular}

\begin{tabular}{|c|c|c|c|c|c|c|c|}
\hline \multicolumn{2}{|c|}{ Ethen / $\mathrm{E}_{83.1} \mathrm{MMA}_{16.9}$} & \multicolumn{2}{|c|}{ Ethen / $\mathrm{E}_{81.5} \mathrm{MMA}_{18.5}$} & \multicolumn{2}{|c|}{ Ethen / $\mathrm{E}_{70.2} \mathrm{MMA}_{29.8}$} & \multicolumn{2}{|c|}{ Ethen / $\mathrm{E}_{64.9} \mathrm{MMA}_{35.1}$} \\
\hline$T / \mathrm{K}$ & $p /$ bar & $T / \mathrm{K}$ & $p /$ bar & $T / \mathrm{K}$ & $p /$ bar & $T / \mathrm{K}$ & $p /$ bar \\
\hline 382.15 & 1465 & 353.15 & 1430 & 370.25 & 1371 & 393.15 & 1500 \\
\hline 391.75 & 1435 & 373.15 & 1309 & 390.35 & 1301 & 413.15 & 1419 \\
\hline 412.55 & 1354 & 393.15 & 1252 & 411.55 & 1246 & 433.15 & 1341 \\
\hline 431.45 & 1292 & 413.15 & 1206 & 430.85 & 1200 & 453.15 & 1286 \\
\hline 451.45 & 1237 & 433.15 & 1176 & 450.75 & 1161 & 473.15 & 1238 \\
\hline \multirow[t]{5}{*}{471.25} & 1208 & 453.15 & 1137 & 470.25 & 1130 & 488.15 & 1202 \\
\hline & & 473.15 & 1108 & 490.45 & 1108 & 508.15 & 1152 \\
\hline & & 488.15 & 1086 & 509.45 & 1076 & & \\
\hline & & 508.15 & 1057 & 529.45 & 1049 & & \\
\hline & & 528.15 & 1039 & & & & \\
\hline
\end{tabular}




\begin{tabular}{|c|c|c|c|c|c|c|c|}
\hline \multicolumn{2}{|c|}{ Ethen / $\mathrm{E}_{58.5} \mathrm{MMA}_{41.5}$} & \multicolumn{2}{|c|}{ Ethen / $\mathrm{E}_{50.4} \mathrm{MMA}_{49.6}$} & \multicolumn{2}{|c|}{ Ethen / $\mathrm{E}_{93.3} \mathrm{BMA}_{6.7}$} & \multicolumn{2}{|c|}{ Ethen / $\mathrm{E}_{88.3} \mathrm{BMA}_{11.7}$} \\
\hline$T / \mathrm{K}$ & $p /$ bar & $T / \mathrm{K}$ & $p /$ bar & $T / \mathrm{K}$ & $p /$ bar & $T / \mathrm{K}$ & $p /$ bar \\
\hline 118.9 & 1673 & 118.9 & 1673 & 374.15 & 1414 & 372.15 & 1315 \\
\hline 129.6 & 1585 & 129.6 & 1585 & 393.15 & 1330 & 393.15 & 1239 \\
\hline 139.1 & 1533 & 139.1 & 1533 & 414.15 & 1264 & 411.15 & 1194 \\
\hline 149.7 & 1478 & 149.7 & 1478 & 434.15 & 1208 & 432.15 & 1161 \\
\hline 169.1 & 1378 & 169.1 & 1378 & 453.15 & 1169 & 451.15 & 1122 \\
\hline 190 & 1306 & 190 & 1306 & 472.15 & 1134 & 470.15 & 1095 \\
\hline 200.3 & 1271 & 200.3 & 1271 & 491.15 & 1106 & 489.15 & 1063 \\
\hline 210.3 & 1242 & 210.3 & 1242 & 513.15 & 1079 & 510.15 & 1039 \\
\hline 220.8 & 1210 & 220.8 & 1210 & 531.15 & 1052 & 531.15 & 1017 \\
\hline
\end{tabular}

\begin{tabular}{|c|c|c|c|c|c|c|c|}
\hline \multicolumn{2}{|c|}{ Ethen / $\mathrm{E}_{81.5} \mathrm{BMA}_{18.5}$} & \multicolumn{2}{|c|}{ Ethen / $\mathrm{E}_{87.3} \mathrm{BMA}_{22.7}$} & \multicolumn{2}{|c|}{ Ethen / $\mathrm{E}_{66.2} \mathrm{BMA}_{33.8}$} & \multicolumn{2}{|c|}{ Ethen / $\mathrm{E}_{56.0} \mathrm{BMA}_{44.0}$} \\
\hline$T / \mathrm{K}$ & $p /$ bar & $T / \mathrm{K}$ & $p /$ bar & $T / \mathrm{K}$ & $p /$ bar & $T / \mathrm{K}$ & $p /$ bar \\
\hline 626.3 & 1082 & 353.15 & 1121 & 350.15 & 1002 & 362.15 & 1064 \\
\hline 646.3 & 1045 & 374.15 & 1085 & 372.15 & 974 & 374.15 & 1038 \\
\hline 666.3 & 1016 & 394.15 & 1054 & 393.15 & 954 & 393.15 & 1023 \\
\hline 686.3 & 993 & 413.15 & 1028 & 413.15 & 940 & 413.15 & 1005 \\
\hline 706.3 & 973 & 432.15 & 1008 & 433.15 & 925 & 432.15 & 995 \\
\hline 726.3 & 958 & 452.15 & 987 & 453.15 & 912 & 452.15 & 969 \\
\hline 746.3 & 946 & 472.15 & 970 & 471.15 & 901 & 471.15 & 957 \\
\hline 761.3 & 930 & 491.15 & 951 & 491.15 & 891 & 490.15 & 952 \\
\hline 781.3 & 915 & 511.15 & 939 & 510.15 & 879 & 511.15 & 938 \\
\hline 801.3 & 901 & 529.15 & 922 & 530.15 & 876 & 531.15 & 924 \\
\hline
\end{tabular}




\begin{tabular}{|c|c|c|c|c|c|c|c|}
\hline \multicolumn{2}{|c|}{ Ethen / $\mathrm{E}_{96.7} \mathrm{BzMA}_{3.3}$} & \multicolumn{2}{|c|}{ Ethen / $\mathrm{E}_{87.3} \mathrm{BzMA}_{12.7}$} & \multicolumn{2}{|c|}{ Ethen $/ \mathrm{E}_{80.0} \mathrm{BzMA}_{20.0}$} & \multicolumn{2}{|c|}{ Ethen / $\mathrm{E}_{74.9} \mathrm{BzMA}_{26.1}$} \\
\hline$T / \mathrm{K}$ & $p /$ bar & $T / \mathrm{K}$ & $p /$ bar & $T / \mathrm{K}$ & $p /$ bar & $T / \mathrm{K}$ & $p /$ bar \\
\hline 389.15 & 1697 & 373.15 & 1903 & 458.15 & 2006 & 458.15 & 2380 \\
\hline 411.15 & 1581 & 393.15 & 1748 & 473.15 & 1830 & 473.15 & 2299 \\
\hline 430.15 & 1501 & 413.15 & 1656 & 483.15 & 1747 & 483.15 & 2230 \\
\hline 450.15 & 1426 & 433.15 & 1579 & 488.15 & 1722 & 493.15 & 2123 \\
\hline 470.15 & 1366 & 453.15 & 1512 & & & 503.15 & 2055 \\
\hline 489.15 & 1311 & 468.15 & 1459 & & & 513.15 & 2014 \\
\hline \multirow[t]{3}{*}{510.15} & 1268 & 488.15 & 1410 & & & 523.15 & 1949 \\
\hline & & 508.15 & 1361 & & & 528.15 & 1881 \\
\hline & & 528.15 & 1302 & & & & \\
\hline
\end{tabular}

\begin{tabular}{|c|c|c|c|c|c|c|c|}
\hline \multicolumn{2}{|c|}{ Ethen / Poly(BMA) } & \multicolumn{2}{|c|}{ Ethen / $\mathrm{E}_{93.0} \mathrm{BMA}_{0.6} \mathrm{MAS}_{6.4}$} & \multicolumn{2}{|c|}{ Ethen / $\mathrm{E}_{93.0} \mathrm{MAS}_{7.0}$} & \multicolumn{2}{|c|}{ Ethen / $\mathrm{E}_{92.7} \mathrm{BMA}_{1.7} \mathrm{MAS}_{5.6}$} \\
\hline$T / \mathrm{K}$ & $p /$ bar & $T / \mathrm{K}$ & $p /$ bar & $T / \mathrm{K}$ & $p /$ bar & $T / \mathrm{K}$ & $p /$ bar \\
\hline 373.15 & 1380 & 475.85 & 2647 & 488.15 & 2620 & 479.15 & 2070 \\
\hline 393.15 & 1320 & 476.15 & 2643 & 493.65 & 2408 & 480.15 & 2076 \\
\hline 413.15 & 1215 & 477.85 & 2584 & 494.65 & 2393 & 493.15 & 1875 \\
\hline 433.15 & 1161 & 481.65 & 2469 & 501.15 & 2203 & 500.15 & 1735 \\
\hline 453.15 & 1147 & 482.55 & 2474 & 502.15 & 2198 & 511.15 & 1680 \\
\hline 473.15 & 1111 & 489.65 & 2269 & 505.15 & 2109 & 520.15 & 1605 \\
\hline 488.15 & 1077 & 489.95 & 2258 & 506.15 & 2089 & & \\
\hline \multirow[t]{4}{*}{508.15} & 1031 & 493.65 & 2160 & 512.15 & 1980 & & \\
\hline & & 493.85 & 2158 & 518.15 & 1872 & & \\
\hline & & 502.15 & 1991 & 522.15 & 1807 & & \\
\hline & & 512.55 & 1810 & 527.15 & 1707 & & \\
\hline
\end{tabular}




\begin{tabular}{|c|c|c|c|c|c|c|c|}
\hline \multicolumn{2}{|c|}{ Ethen / $\mathrm{E}_{92.7} \mathrm{BMA}_{5.5} \mathrm{MAS}_{1.8}$} & \multicolumn{2}{|c|}{ Ethen / $\mathrm{E}_{92.9} \mathrm{BMA}_{4.1} \mathrm{MAS}_{2.9}$} & \multicolumn{2}{|c|}{ Ethen / $\mathrm{E}_{92.7} \mathrm{BMA}_{3.5} \mathrm{MAS}_{3.8}$} & \multicolumn{2}{|c|}{ Ethen $/ \mathrm{E}_{92.8} \mathrm{MAS}_{7.2}$} \\
\hline$T / \mathrm{K}$ & $p /$ bar & $T / \mathrm{K}$ & $p /$ bar & $T / \mathrm{K}$ & $p /$ bar & $T / \mathrm{K}$ & $p /$ bar \\
\hline 373.15 & 2130 & 433.05 & 2195 & 435.15 & 2650 & 495.55 & 2380 \\
\hline 393.15 & 1860 & 445.95 & 2012 & 439.15 & 2499 & 496.35 & 2485 \\
\hline 413.15 & 1645 & 452.25 & 1904 & 451.15 & 2264 & 497.35 & 2366 \\
\hline 433.15 & 1460 & 452.95 & 1888 & 461.65 & 2054 & 502.25 & 2252 \\
\hline 443.85 & 1366 & 465.15 & 1678 & 462.15 & 2047 & 502.65 & 2257 \\
\hline 445.15 & 1374 & 471.15 & 1590 & 471.45 & 1915 & 503.35 & 2224 \\
\hline 451.95 & 1315 & 472.15 & 1580 & 480.55 & 1789 & 507.15 & 2160 \\
\hline 454.65 & 1301 & 481.55 & 1481 & 481.15 & 1780 & 507.35 & 2151 \\
\hline 465.25 & 1247 & 481.85 & 1472 & 489.85 & 1682 & 507.85 & 2137 \\
\hline 466.15 & 1246 & 482.55 & 1477 & 490.15 & 1666 & 513.95 & 2032 \\
\hline 471.65 & 1212 & 492.55 & 1383 & 502.15 & 1540 & 514.35 & 2014 \\
\hline 487.15 & 1136 & 493.15 & 1387 & 509.55 & 1472 & 515.45 & 2001 \\
\hline 490.75 & 1127 & 500.85 & 1323 & 509.95 & 1468 & 518.45 & 1951 \\
\hline 491.15 & 1138 & 501.85 & 1327 & 521.15 & 1377 & 519.45 & 1943 \\
\hline 506.65 & 1078 & 513.85 & 1258 & & & 520.15 & 1981 \\
\hline 511.15 & 1066 & 514.15 & 1258 & & & 524.15 & 1912 \\
\hline \multirow[t]{3}{*}{527.15} & 1030 & 521.85 & 1225 & & & 529.15 & 1837 \\
\hline & & 531.15 & 1223 & & & 533.15 & 1780 \\
\hline & & 532.15 & 1180 & & & & \\
\hline
\end{tabular}




\begin{tabular}{|c|c|c|c|c|c|c|c|}
\hline \multicolumn{2}{|c|}{ Ethen / $\mathrm{E}_{93} \mathrm{BMA}_{0.9} \mathrm{MAS}_{6.1}$} & \multicolumn{2}{|c|}{ Ethen / $\mathrm{E}_{93.0} \mathrm{BMA}_{0.4} \mathrm{MAS}_{6.6}$} & \multicolumn{2}{|c|}{ Ethen / $\mathrm{E}_{92.9} \mathrm{BMA}_{6.8} \mathrm{MAS}_{0.3}$} & \multicolumn{2}{|c|}{ Ethen / $\mathrm{E}_{92.9} \mathrm{BMA}_{6.2} \mathrm{MAS}_{0.9}$} \\
\hline$T / \mathrm{K}$ & $p /$ bar & $T / \mathrm{K}$ & $p /$ bar & $T / \mathrm{K}$ & $p /$ bar & $T / \mathrm{K}$ & $p /$ bar \\
\hline 470.85 & 2608 & 474.15 & 2750 & 354.15 & 1626 & 373.15 & 1717 \\
\hline 471.55 & 2566 & 483.15 & 2505 & 363.65 & 1546 & 374.15 & 1698 \\
\hline 477.65 & 2455 & 489.75 & 2328 & 372.35 & 1486 & 381.55 & 1628 \\
\hline 478.55 & 2372 & 490.15 & 2306 & 382.55 & 1426 & 381.85 & 1625 \\
\hline 478.75 & 2364 & 491.75 & 2283 & 383.05 & 1420 & 392.15 & 1529 \\
\hline 482.25 & 2292 & 492.45 & 2260 & 391.15 & 1379 & 392.65 & 1522 \\
\hline 483.05 & 2268 & 492.55 & 2244 & 391.65 & 1378 & 403.95 & 1448 \\
\hline 487.15 & 2168 & 493.15 & 2240 & 394.45 & 1361 & 412.15 & 1405 \\
\hline 488.05 & 2148 & 497.65 & 2147 & 411.65 & 1288 & 429.05 & 1340 \\
\hline 491.65 & 2076 & 500.85 & 2071 & 412.35 & 1285 & 430.05 & 1335 \\
\hline 492.25 & 2057 & 501.45 & 2053 & 428.75 & 1229 & 431.55 & 1322 \\
\hline 497.45 & 1952 & 502.45 & 2035 & 430.45 & 1223 & 432.05 & 1319 \\
\hline 501.95 & 1888 & 507.95 & 1946 & 443.15 & 1190 & 445.15 & 1272 \\
\hline 503.05 & 1861 & 508.25 & 1932 & 444.45 & 1188 & 446.05 & 1267 \\
\hline 506.55 & 1813 & 512.25 & 1869 & 451.95 & 1170 & 453.15 & 1236 \\
\hline 508.05 & 1786 & 512.55 & 1866 & 464.65 & 1144 & 453.55 & 1240 \\
\hline 508.25 & 1781 & 517.65 & 1795 & 473.15 & 1126 & 461.15 & 1218 \\
\hline 512.25 & 1737 & 521.05 & 1742 & 485.35 & 1104 & 471.15 & 1196 \\
\hline 512.55 & 1728 & 521.15 & 1742 & 492.35 & 1092 & 471.95 & 1192 \\
\hline 512.75 & 1720 & & & 511.25 & 1064 & 480.45 & 1171 \\
\hline 517.35 & 1678 & & & & & 481.75 & 1168 \\
\hline 518.15 & 1668 & & & & & 490.75 & 1149 \\
\hline 518.25 & 1672 & & & & & 491.85 & 1147 \\
\hline 522.15 & 1626 & & & & & 500.65 & 1127 \\
\hline 523.15 & 1613 & & & & & 518.15 & 1097 \\
\hline 527.05 & 1573 & & & & & 520.15 & 1096 \\
\hline 528.05 & 1554 & & & & & & \\
\hline 528.25 & 1556 & & & & & & \\
\hline
\end{tabular}




\begin{tabular}{|c|c|c|c|c|c|c|c|}
\hline \multicolumn{2}{|c|}{ Propen / Poly(MA) } & \multicolumn{2}{|c|}{ Propen / Poly(MMA) } & \multicolumn{2}{|c|}{ Ethen / $\mathrm{E}_{92.9} \mathrm{BMA}_{0.8} \mathrm{MAS}_{6.3}$} & \multicolumn{2}{|c|}{ Ethen / $\mathrm{E}_{92.9} \mathrm{BMA}_{0.8} \mathrm{MAS}_{6.3}$} \\
\hline$T / \mathrm{K}$ & $p /$ bar & $T / \mathrm{K}$ & $p /$ bar & $T / \mathrm{K}$ & $p /$ bar & $T / \mathrm{K}$ & $p /$ bar \\
\hline 473.15 & 2750 & 427.15 & 2700 & 467.15 & 2707 & 273.15 & 2333 \\
\hline 480.15 & 2465 & 433.15 & 2379 & 472.15 & 2565 & 273.15 & 2263 \\
\hline 481.15 & 2418 & 433.65 & 2356 & 478.15 & 2429 & 273.15 & 2160 \\
\hline 482.15 & 2391 & 443.35 & 2060 & 483.15 & 2327 & 273.15 & 2080 \\
\hline 483.15 & 2361 & 444.15 & 2027 & 490.15 & 2222 & 273.15 & 1812 \\
\hline 491.15 & 2109 & 453.15 & 1891 & 492.15 & 2175 & 273.15 & 1738 \\
\hline 493.75 & 2018 & 461.15 & 1739 & 498.15 & 2062 & & \\
\hline 501.15 & 1850 & 462.15 & 1728 & 504.15 & 1972 & Ethen / $E_{8}$ & ${ }_{5.5} \mathrm{MAS}_{7.3}$ \\
\hline 510.15 & 1691 & 462.65 & 1712 & 514.15 & 1845 & $T / \mathrm{K}$ & $p /$ bar \\
\hline 512.75 & 1641 & 471.15 & 1577 & 523.15 & 1762 & 486.15 & 2530 \\
\hline 521.95 & 1513 & 471.85 & 1573 & 530.15 & 1681 & 494.15 & 2324 \\
\hline 523.45 & 1487 & 472.35 & 1563 & & & 498.15 & 2231 \\
\hline 525.45 & 1465 & 481.15 & 1458 & & & 504.15 & 2123 \\
\hline 526.45 & 1457 & 490.65 & 1364 & & & 508.15 & 2041 \\
\hline \multirow[t]{4}{*}{531.65} & 1399 & 502.15 & 1265 & & & 513.15 & 1944 \\
\hline & & 511.15 & 1204 & & & 519.15 & 1873 \\
\hline & & 523.15 & 1122 & & & 528.15 & 1779 \\
\hline & & 532.15 & 1078 & & & 532.15 & 1750 \\
\hline
\end{tabular}


Trübungsdrücke für ternäre Mischungen aus Ethen / Comonomer / Copolymer bei $200^{\circ} \mathrm{C}$

\begin{tabular}{|cc|cc|}
\hline \multicolumn{2}{|c|}{ Ethen / BzMA / E $_{86.9} \mathrm{BzMA}_{13.3}$} & \multicolumn{2}{c|}{ Ethen / BzMA / $\mathrm{E}_{79.7} \mathrm{BzMA}_{20.3}$} \\
\hline$w_{\mathrm{BzMA}}[$ Gew.\%] & $p /$ bar & $w_{\text {BzMA }}[\mathrm{Gew} \%]$ & $p /$ bar \\
\hline 3.67 & 1339 & 6.32 & 1401 \\
5.92 & 1308 & 8.44 & 1368 \\
8.07 & 1277 & 10.5 & 1330 \\
10.1 & 1245 & 12.4 & 1299 \\
12.1 & 1227 & 14.3 & 1275 \\
\hline
\end{tabular}

\begin{tabular}{|cc|cc|}
\hline \multicolumn{2}{|c|}{ Ethen / BzMA / E $_{74.8} \mathrm{BzMA}_{25.2}$} & \multicolumn{2}{c|}{ Ethen / BzMA / $\mathrm{E}_{69.6} \mathrm{BzMA}_{30.4}$} \\
\hline$w_{\text {BzMA }}[$ Gew.\%] & $p /$ bar & $w_{\text {BzMA }}[\mathrm{Gew} \%]$ & $p /$ bar \\
\hline 8.71 & 1463 & 11.7 & 1508 \\
10.7 & 1425 & 13.6 & 1460 \\
12.7 & 1380 & 15.4 & 1417 \\
14.5 & 1352 & 17.2 & 1375 \\
16.3 & 1315 & 18.8 & 1340 \\
\hline
\end{tabular}

\begin{tabular}{|cc|cc|}
\hline \multicolumn{2}{|c|}{ Ethen / BzMA / $\mathrm{E}_{76.5} \mathrm{BzMA}_{33.5}$} & \multicolumn{2}{c|}{ Ethen / EEMA / E88.6 $\mathrm{EMA}_{11.4}$} \\
\hline$w_{\text {BzMA }}[$ Gew.\%] & $p /$ bar & $w_{\text {EMA }}[$ Gew.\%] & $p /$ bar \\
13.7 & 1515 & 3.48 & 1034 \\
15.5 & 1472 & 5.48 & 1004 \\
17.2 & 1430 & 7.40 & 985 \\
18.9 & 1393 & 9.25 & 973 \\
20.5 & 1352 & 11.0 & 955 \\
\hline
\end{tabular}




\begin{tabular}{|cc|cc|}
\hline Ethen / EEMA / $\mathrm{E}_{80.8} \mathrm{EMA}_{19.2}$ & \multicolumn{2}{|c|}{ Ethen / EEMA / F $_{73.9} \mathrm{EMA}_{26.1}$} \\
\hline$w_{\text {EMA }}[$ Gew.\%] & $p /$ bar & $w_{\mathrm{EMA}}[\mathrm{Gew} . \%]$ & $p /$ bar \\
\hline 5.82 & 978 & 7.94 & 940 \\
7.72 & 960 & 9.76 & 925 \\
9.55 & 935 & 11.5 & 906 \\
11.3 & 920 & 13.2 & 886 \\
13.0 & 890 & 14.8 & 865 \\
\hline
\end{tabular}

\begin{tabular}{|cc|cc|}
\hline \multicolumn{2}{|c|}{ Ethen / EEMA / E ${ }_{62} \mathrm{EMA}_{38}$} & \multicolumn{2}{c|}{ Ethen / EEMA / $\mathrm{E}_{71} \mathrm{EMA}_{29}$} \\
\hline$w_{\text {BzMA }}[$ Gew.\%] & $p /$ bar & $w_{\text {EMA }}[$ Gew.\%] & $p /$ bar \\
\hline 14.2 & 885 & 11.3 & 915 \\
15.8 & 871 & 13.0 & 896 \\
17.3 & 853 & 14.6 & 879 \\
18.7 & 823 & 16.1 & 853 \\
20.1 & 803 & 17.6 & 833 \\
\hline
\end{tabular}




\section{Anhang D}

Auflistung der für die PC-SAFT-Modellierungen verwendeten Reinstoff- und binären Parameter

\section{Reinstoffparameter}

\begin{tabular}{|c|c|c|c|c|c|}
\hline \multirow[b]{2}{*}{ Polymer } & \multirow{2}{*}{$\begin{array}{l}\mathrm{m} / \mathrm{M}^{\mathrm{b}} \\
\mathrm{mol} \cdot \mathrm{g}^{-1}\end{array}$} & \multirow{2}{*}{$\begin{array}{l}\sigma \\
\AA\end{array}$} & \multirow{2}{*}{$\begin{array}{c}\varepsilon / k \\
\mathrm{~K}\end{array}$} & \multicolumn{2}{|c|}{ binäres System } \\
\hline & & & & Lösungsmittel & Referenz \\
\hline Polyethylen (LDPE) ${ }^{a}$ & 0.0263 & 4.02 & 249.5 & Ethen & {$[\mathrm{A}]$} \\
\hline Poly(MA) & 0.0309 & 3.50 & 243 & Propen & diese Arbeit \\
\hline Poly(EA) & 0.0271 & 3.65 & 229 & Ethen & diese Arbeit \\
\hline Poly $(\mathrm{PA})^{\mathrm{c}}$ & 0.0262 & 3.80 & 225 & Ethen & diese Arbeit \\
\hline Poly(BA) & 0.0259 & 3.95 & 224 & Ethen & {$[\mathrm{B}]$} \\
\hline Poly(MMA) & 0.0262 & 3.60 & 245 & Propen & diese Arbeit \\
\hline Poly(BMA) & 0.0268 & 3.75 & 233.8 & Ethen & diese Arbeit \\
\hline
\end{tabular}

${ }^{\mathrm{a}}$ J. Gross, G. Sadowski, Ind. Eng. Chem. Res. 41 (2002) 1084

${ }^{\mathrm{b}}$ Die Segmentzahl $m$ wird durch Multiplikation des Quotienten $(m / M)$ mit der Molmasse $M$ erhalten.

${ }^{c}$ Die Reinstoffparameter für Polypropylacrylat wurden durch Interpolation der Reinstoffparameter von Poly(MA), Poly(EA) und Poly(BA) bestimmt (vgl. Abschnitt 6.2)

Ref. [A]: C. Müller, Dissertation Karlsruhe (1998)

Ref. [B]: H. S. Buyn, B, M. Hasch, M. A. McHugh, F. O. Mähling, M. Busch, M. Buback, Macromolecules 29 (1996) 1625

\section{Binäre Parameter}

\begin{tabular}{ccc}
\hline \hline Lösungsmittel / Copolymer & Segment-Kombination & binäre Parameter \\
\hline \multirow{2}{*}{ Ethen / E-MA } & Ethen - LDPE & 0.040 \\
& Ethen - Poly(MA) & 0.020 \\
& Polyethylen Segment - Poly(MA) Segment & $0.01161 \cdot F_{\text {MA }}+0.04006$ \\
\hline \multirow{2}{*}{ Ethen / E-EA } & Ethen - LDPE & 0.040 \\
& Ethen - Poly(EA) & 0.0135 \\
& Polyethylen Segment - Poly(EA) Segment & 0.020 \\
\hline \multirow{2}{*}{ Ethen / E-PA } & Ethen - LDPE & 0.040 \\
& Ethen - Poly(PA) & 0.0225 \\
Ethen / E-BA & Polyethylen Segment - Poly(PA) Segment & 0.010 \\
& Ethen - Poly(BA) & 0.040 \\
Ethen / E-MMA & Polyethylen Segment - Poly(BA) Segment & 0.030 \\
& Ethen - LDPE & 0 \\
\hline \multirow{2}{*}{ Ethen / E-BMA } & Ethen - Poly(MMA) & 0.040 \\
& Polyethylen Segment - Poly(MMA) Segment & 0.028 \\
& Polyen - LDPE & $0.06446 \cdot F_{\text {MMA }}+0.03002$ \\
\hline Propen / Homopolymer & Ethen - Poly(BMA) & 0.040 \\
& Propen - Poly(MA) & 0.020 \\
\hline \hline
\end{tabular}




\section{Lebenslauf}

Henning Latz

10.08.1969

geboren in Düren (Eifel)

verheiratet

\section{Schulausbildung}

$1975-1981$

Grundschule und Orientierungsstufe in Schierbrok und Bookholzberg (Oldenburg)

$1981-1988$

Gymnasium Ganderkesee (Oldenburg)

Mai 1988

Allgemeinen Hochschulreife

\section{Wehrdienst}

Okt. 1988 - Dez. 1989

Grundwehrdienst in Wildeshausen, Delmenhorst und Nienburg

\section{Hochschulstudium}

April 1990 - Juli 1999

Studium der Chemie an der Georg-August-Universität Göttingen

Febr. 1995

Diplomvorprüfung

Juni 1998 - Mai 1999

Juli 1999

seit Juli 1999

Diplomarbeit am Institut für Physikalische Chemie unter Anleitung von Herrn Prof. Dr. M. Buback: „Entmischungsverhalten der Systeme Ethen-Poly(Ethen-co-Acrylsäure) und EthenPoly(Ethen-co-Methacrylsäure) bis $\mathrm{zu}$ hohen Drücken und Temperaturen“"

Diplomprüfung

Anfertigung einer Dissertation am Institut für Physikalische Chemie der Georg-August Universität Göttingen unter Anleitung von Hern Prof. Dr. M. Buback

Juli 1998 - Juli 1999

Studentische Hilfskraft am Institut für Physikalische Chemie der Georg-August-Universität Göttingen

Aug. 1998 - Dez. 1999 Wissenschaftliche Hilfskraft am Institut für Physikalische Chemie

Seit Jan 2000

Wissenschaftlicher Angestellter am Institut für Physikalische Chemie

Jan. 2000 - Dez. 2001

Mitarbeit im DFG-Schwerpunkt „Stoffeigenschaften komplexer fluider Gemische“"

seit Mai 2000

Mitglied im Europäischen Graduiertenkolleg „Microstructural Control in Free-Radical Polymerization“" 



\section{DANKSAGUNG}

An erster Stelle bedanke ich mich herzlich bei meinem Doktorvater Herrn Professor Dr. M. Buback für die wohlwollende Förderung und großzügige Unterstützung dieser Arbeit. Darüber hinaus bin ich Herrn Buback sehr dankbar dafür, dass er es mir ermöglichte, an herausfordernden Industriekooperationen mitzuarbeiten.

Meinen Kollegen Matthias Müller, Florian Becker, Stefan Jauer und Stefan Hinrichs danke ich für die vielfach bewiesene freundschaftliche Hilfsbereitschaft und ihr großes Engagement bei Auftragsmessungen. Matthias Müller bin ich zudem für die Durchführung unzähliger NMR-Experimente und die gemeinsame Arbeit bei Umbau und Instandhaltung von Minitechnikum I sehr zu Dank verpflichtet. An die gute Zusammenarbeit an den Mini-Technika mit all ihren Besonderheiten und den Teamgeist dabei werde ich mich immer gerne erinnern. Allen anderen Mitarbeitern des Arbeitskreises danke ich für die angenehme und oft humorerfüllte Arbeitsatmosphäre - besonders denke ich dabei an Fabian Günzler, Nuria Garcia und Mark Egorov.

Heike Rohmann und den studentischen Mitarbeitern Olaf Janssen und Jens Diese sei für ihre versierte praktische Hilfe bei Hochdruckmessungen und Umbauten gedankt.

Herrn Ingenieur V. Meyer und den Mitarbeitern der Institutswerkstätten sowie im speziellen Herrn W. Noack möchte ich für die hervorragende technische Unterstützung und die zügige Bearbeitung von Fertigungs- und Reparaturaufträgen danken. Herrn Dr. H.P. Vögele gilt mein Dank für viele praktische Hinweise und prompte Hilfsbereitschaft bei Problemen mit Spektrometern und anderen Geräten

Der Arbeitsgruppe von Frau Professor Dr. G. Sadowski in Dortmund und dabei besonders Feelly Tumakaka danke ich sehr herzlich für die freundliche Aufnahme im Herbst 2002 und die Einweisung in die Modellierungen mit PC-SAFT.

Der BASF AG, insbesondere Herrn Dr. F.-O. Mähling, danke ich für die Unterstützung in Form von Ethenlieferungen sowie das Interesse an den Ergebnissen unserer Arbeiten bei vielen Arbeitstreffen.

Der Deutschen-Forschungs-Gesellschaft bin für die finanzielle Unterstützung im Rahmen des Schwerpunktprogramms "Stoffeigenschaften komplexer fluider Gemische" zu Dank verpflichtet.

Besondere Dankbarkeit empfinde ich gegenüber meiner Frau Swantje und meinen Eltern für ihren unerschöpflichen Rückhalt. 